\title{
Controlled delamination of metal films by hydrogen loading
}

\author{
Dissertation \\ zur Erlangung des Doktorgrades \\ der Mathematisch-Naturwissenschaftlichen Fakultäten \\ der Georg-August-Universität Göttingen
}

vorgelegt von

Eugen Nikitin

aus

Ust-Kamenogorsk (Kasachstan)

Göttingen 2008 
D 7

Referent:

Prof. Dr. R. Kirchheim

Korreferent:

Prof. Dr. A. Pundt

Tag der mündlichen Prüfung:

18.11.2008 


\section{Table of Contents}

Introduction $\quad 1$

1. Controlled buckling method 3

1.1 Theory and actual methods for adhesion energy determination $\ldots \ldots \ldots \ldots \ldots$

1.2 Controlled buckling method for adhesion energy calculation $\ldots \ldots \ldots \ldots \ldots 8$

1.2.1 The idea of this method $\ldots \ldots \ldots \ldots \ldots \ldots \ldots \ldots$

1.2.2 Controlled hydrogen absorption, electrochemical method . . . . . . . 12

1.2.3 Optical microscopy studies and stress measurement . . . . . . . . . 14

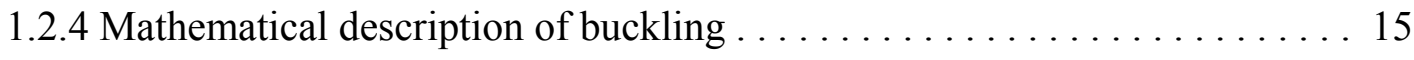

2. Experimental techniques 22

2.1 Sample preparation by sputtering techniques $\ldots \ldots \ldots \ldots \ldots \ldots \ldots \ldots \ldots \ldots \ldots$

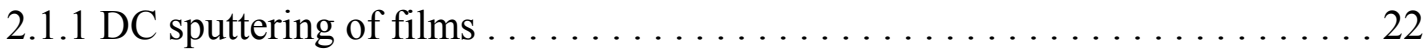

2.1.2. Magnetron sputtering $\ldots \ldots \ldots \ldots \ldots \ldots \ldots \ldots \ldots \ldots \ldots \ldots \ldots \ldots \ldots \ldots$

2.2 EMF measurement during hydrogen loading . . . . . . . . . . 23

2.3 Bending setup. Two cells for controlled hydrogen loading . . . . . . . . 24

2.4 Buckles observation and determination of their shape for adhesion determination.

Shape of buckles as a measure of adhesion . . . . . . . . . . . . . 27

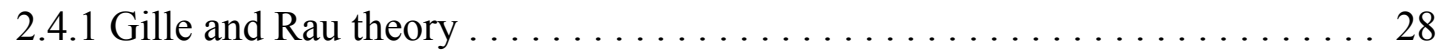

2.4.2 White-light interferometry . . . . . . . . . . . . . . . . . 29

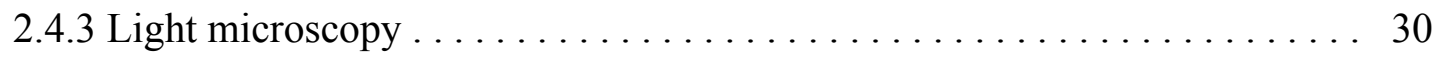

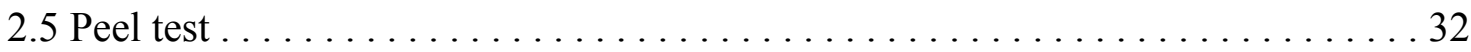

3. Experimental results 33

3.1 Hydrogen loading of $\mathrm{Pd} / \mathrm{Nb} / \mathrm{Pd}$ films on polycarbonate $\ldots \ldots \ldots \ldots \ldots \ldots 33$

3.1.1 Buckle formation at the critical hydrogen concentration ........... 33

3.1.2 Influence of film thickness on critical concentration and critical stress . . . 36

3.1.3 Substrate thickness impact . . . . . . . . . . . . . . . . . 40

3.1.4 Continuous hydrogen loading $\ldots \ldots \ldots \ldots \ldots \ldots \ldots \ldots \ldots \ldots \ldots \ldots \ldots \ldots$

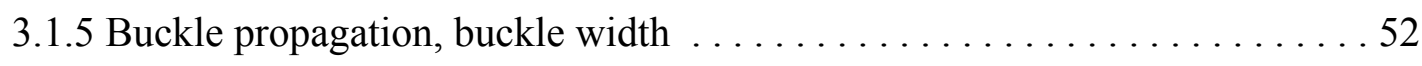

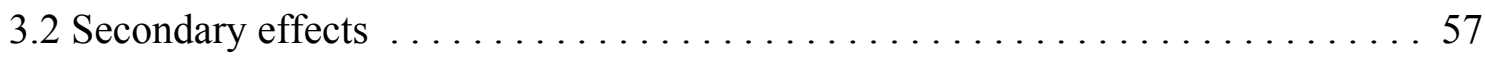

3.2.1 Effect of electrolyte on the stress measurement experiments . . . . . . . 57

3.2.2 Polymer relaxation during buckling process $\ldots \ldots \ldots \ldots \ldots \ldots \ldots \ldots$

3.2.3 Buckle formation observed in the stress curve $\ldots \ldots \ldots \ldots \ldots \ldots 6$

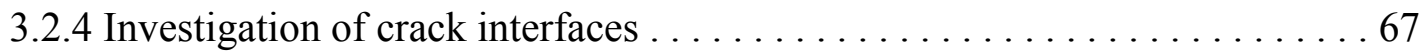

3.3 Influence of film delamination on the chemical potential of hydrogen . . . . . 68 
3.4 Adhesion energy calculations, results for different interface layers. Alternative methods for adhesion energy calculation: Gille and Rau formula and peel test . . 73 3.4.1 Adhesion energy calculation for $\mathrm{Pd} / \mathrm{Nb} / \mathrm{Pd}$ films on $\mathrm{PC} \ldots \ldots \ldots \ldots \ldots 73$

3.4.2 Influence of PC plasma pre-treatment on the adhesion energy values . . . . . 78

3.4.3 Stress measurement during hydrogen loading and adhesion energy calculation

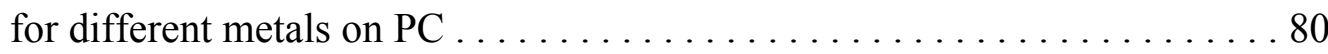

3.4.4 Adhesion energy measurements by means of white-light interferometry . . . 83

3.4.5 Comparative measurements at the model system $\mathrm{Pd} / \mathrm{Nb} / \mathrm{Pd} / / \mathrm{PC}$ by determination of the peel strength $\ldots \ldots \ldots \ldots \ldots \ldots \ldots \ldots \ldots \ldots$

3.4.6 Buckling of $\mathrm{Pd} / \mathrm{Nb} / \mathrm{Pd}$ film on $\mathrm{PC}$ observed by $\mathrm{X}$-ray irradiation of the

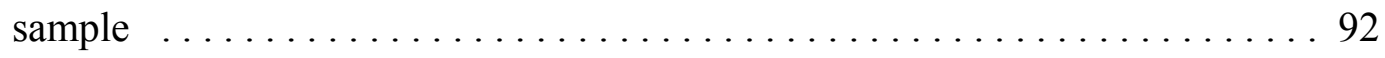

3.5 Hydrogen loading of niobium films on sapphire substrates $\ldots \ldots \ldots \ldots \ldots 9$

3.5.1 Hydrogen loading of $800 \mathrm{~nm} \mathrm{Nb}$ film on sapphire .............. 94

3.5.2 Hydrogen loading of $1.95 \mu \mathrm{m}$ and $2.5 \mu \mathrm{m} \mathrm{Nb}$ film on sapphire . . . . . . . 97

3.5.3 Hydrogen loading of $4 \mu \mathrm{m}$ and $4.75 \mu \mathrm{m} \mathrm{Nb}$ film on sapphire . . . . . . . . 102

3.5.4 Adhesion energy between $\mathrm{Nb}$ and sapphire . . . . . . . . . . . 105

3.5.5 Influence of stress in $\mathrm{Nb}$ films on hydride formation . . . . . . . . . 108

4. Discussion 111

4.1 Hydrogen loading of model films on polycarbonate $\ldots \ldots \ldots \ldots \ldots \ldots \ldots \ldots 11$

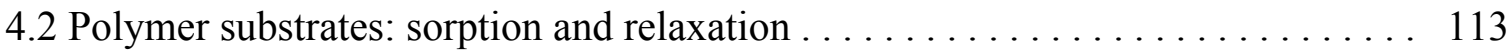

4.3 Adhesion energy calculations of different interface layers using the controlled

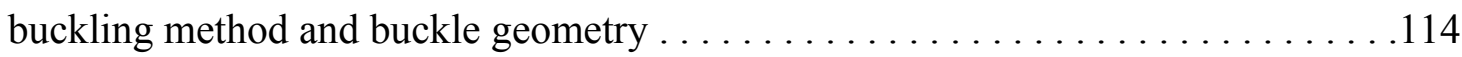

4.4 Investigation of $\mathrm{Nb}$ /sapphire bonding by using of controlled buckling technique .116 4.5 Single buckling events during hydrogen loading $\ldots \ldots \ldots \ldots \ldots \ldots \ldots \ldots$

4.6 Hydrogen loading of niobium films on sapphire $\ldots \ldots \ldots \ldots \ldots \ldots \ldots \ldots \ldots$

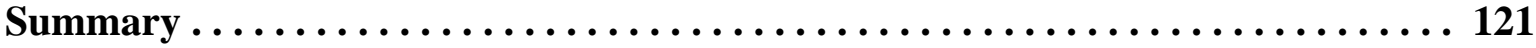

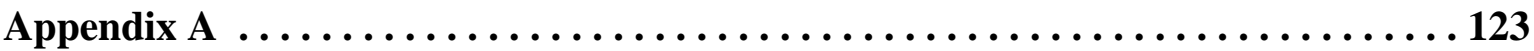

A.1 Morphological changes of $\mathrm{Nb}$ surface during hydrogen loading . . . . . . . 123

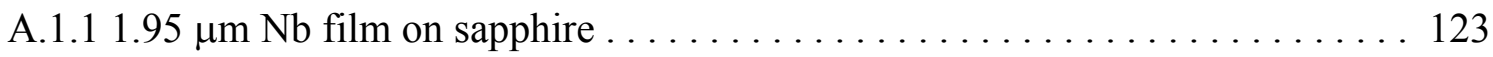

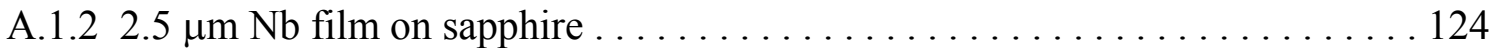

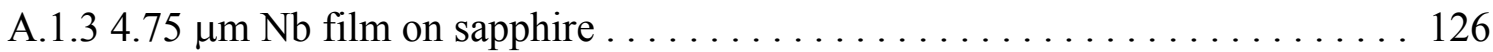

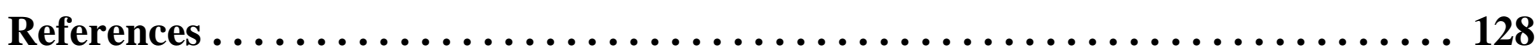




\section{Introduction}

Thin metal films and coatings on substrates are widely used in electronic industry, machine building, jewel industry, etc. as protective and electroconductive materials. A good quality of bonding is important for the successful operation. Such systems are complex with characteristic properties resulting from boundary and surface effects. The quantitative measurement of the bonding energy requires the understanding of detachment, failure mechanisms and the ability to separate the films from their substrates in a controlled way.

High mechanical stress between a film and its substrate often leads to local or global film detachment in thin film samples or coatings. The occurring stress often results from film oxidation, different thermal expansion coefficients of film and substrate or other mechanisms. Knowledge on the nature of these processes and the application of mathematical models describing these processes are necessary for the investigation of the adhesion between two materials. Film detachment occurs above a critical in plane stress and is due to crack formation at the interface between the film and the substrate leading to buckling that is often observed directly after sample preparation. Two typical buckle morphologies, distinguished in literature [M04], are telephone cord buckles and straight-sided buckles. The transition mode between these two is a varicose mode. The mode of buckle appearance depends on the properties of the film-substratesystem, such as the film thickness, stress, interface adhesion and Poisson's ratio of the film.

The appearance of buckles in many technological applications has to be avoided. But on the contrary buckling can be applied to study the physical properties of the film-substrate-system. Interface regions with poor adhesion can be detected, for example, or the strength of bonding between two materials can be measured.

In this work a new technique is presented and investigated, applicable for the determination of the adhesion energy between a metal film and a substrate. In this method solute induced stress in a metal film is applied, which is achieved by hydrogen absorption of the film. If the film is clamped to a rigid substrate, during hydrogen absorption high in-plane compressive stresses up to several GPa [Lau98] occur. These stresses are large enough to lead to film detachment from the substrate. To apply this technique, just an optical microscope is needed in a first-order approximation, allowing the detection of the onset of buckling. To relate buckle formation to the stress stored in the system, equipment for the stress measurement is needed. As the stress results in a substrate bending, it can therefore be measured by a displacement transducer, laser beam deflection devices, etc. To investigate this technique the system of metal-polymer was used, because of a great importance of such systems in our life. Industrially, metal films deposited on a 
polymer are widely used for gas barrier applications, photoconductive and composite materials, for decorative purposes, in the automotive industry, etc. The most polymers used as commercial materials have a low surface energy and as a result a low adhesion to high surface energy metallic coatings. The realization and reproducibility of good adhesion for metallic coatings on polymer substrates is an important industrial and scientific problem. The interaction between a metal and a polymer strongly depends on the type of the metal and on the functional groups present in the polymer. There are some techniques most commonly used to promote the adhesion of polymers to metals. Some of these are oxidation of polymer substrate surfaces, chemical modification of the interfacial polymer chains, and plasma treatment of the metallic surfaces. It is very important to test the effect of such techniques and to determine quantitatively the bonding between a polymer and a metal.

This work is divided into four chapters. In chapter one a short overview of conventional methods of adhesion energy investigation will be described. The presentation of the new technique, which is called a controlled buckling method for adhesion energy determination, will be given. The idea of this method and the mathematical model for adhesion energy derivation is presented. The setup, which is needed to perform this method, will be described. Thereby, some aspects of electrochemical hydrogen loading will be considered.

Details about the experimental techniques and set-ups will be presented in chapter two.

In chapter three experimental results will be shown on the hydrogen loading of metal films on polymer substrates which result in film delamination and buckle formation. Physical aspects such as the influence of film and substrate thickness on the critical hydrogen concentration for buckling as well as the impact of the electrolyte are discussed. In addition, the crack interface was analysed with SIMS. Adhesion energies of different metals on different pre-treated PC were measured. The results were compared with the adhesion energies obtained from the geometry of buckles using the theory of Gille and Rau [GR84]. Hydrogen loading from the gas phase was also applied to compare the stress evolution with that determined by electrochemical loading. Also the niobium/sapphire interface, whose extraordinary adhesion is well-known, was investigated. Chapter four includes a discussion on the results and treats major effects.

It will be shown in this work that hydrogen loading of thin films is a powerful tool to detach a film from a substrate in a controlled way and to measure the adhesion energy, if all important effects are understood and taken into account. 


\section{Controlled buckling method}

In chapter 1.1 known theoretical aspects of adhesion and practical methods of adhesion measuring are described. In chapter 1.2 a new method of adhesion testing by using controlled hydrogen loading is presented. The idea, the mathematical description and the physical aspects of this method are investigated.

\subsection{Theory and actual experimental methods for adhesion energy determination}

Adhesion is defined by the American Society for Testing and Materials as "the state in which two surfaces are held together by interfacial forces which may consist of valence forces or interlocking forces, or both" [ASTM].

The adhesion refers to two bodies holding together by a range of different forces that are acting across their contact area on a molecular level. Basically, the energetic state of the two contacted surfaces will determine the magnitude of adhesion.

Many theoretical models of adhesion mechanisms have been proposed. Following Siau, the main theories are summarized as follows [S05]:

1. Mechanical interlocking: Penetration of an adhesive into the microscopic asperities (i.e. cavities, hills, pores) of the other material is considered to be the main factor in determining adhesive strength.

2. Electronic theory: Electron transfer leads to generation of a double layer at the interface, resulting in the attractive forces across the electrical double layer.

3. Theory of boundary layers and interfaces: A boundary layer or interfacial zone is formed which properties are differing from the bulk properties, causing cohesive failures.

4. Adsorption (thermodynamic theory): Attractive forces at the interface are a result of Van der Waals interactions.

5. Diffusion theory: Used mainly for adhesion of polymers to themselves and to each other.

6. Chemical bonding theory: The formation of ionic and covalent bonds between both types of materials is considered.

Any of the six mechanisms of adhesion or their combinations can be responsible for the material bonding, but in most situations the adsorption theory prevails.

Mittal [M95] distinguishes three types of adhesion that can be studied: fundamental (intrinsic), thermodynamic and practical adhesion. For the evaluation of the first type of adhesion it is necessary to know the type of interaction and the number of interacting centres per unit area. The 
summation of all interfacial intermolecular interactions between the contacting materials gives the fundamental adhesion.

From a thermodynamic point of view the work of adhesion of the interface is the change of free energy when an interface is formed:

$$
\gamma_{A}=\gamma_{f}+\gamma_{S}-\gamma_{f S}
$$

where $\gamma_{f}$ and $\gamma_{S}$ are the specific surface energies of the film and the substrate, respectively and $\gamma_{f S}$ is the energy of the interface. $\gamma_{A}$ is usually called the (true) work of adhesion or true (thermodynamic) adhesion energy. The work of adhesion is an intrinsic property of the filmsubstrate-system and depends on the type of bonding and the level of initial surface contamination. The surfaces of inorganic solids such as metals exhibit a high surface energy; polymer surfaces possess a much lower surface energy [VRS98].

The practical work of adhesion signifies the work required to remove or detach a film from the substrate. The terminology of the practical work of adhesion is very different in literature. Terms like the total work of fracture, fracture energy, macroscopic work of fracture, total energy input, crack extension force and interfacial toughness can be found.

A common technique to determine the work of adhesion are contact angle measurements in wetting experiments [LCE98]. If the tested material particle is in mechanical equilibrium on a substrate, then:

$$
\gamma_{f S}=\gamma_{S}-\gamma_{f} \cdot \cos \Theta
$$

where $\Theta$ is the contact angle between the particle free surface and the substrate as shown in Fig. 1.1.

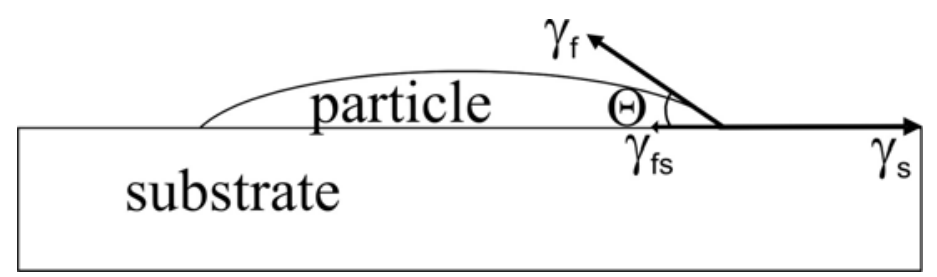

Figure 1.1: contact angle measurement (schematically) for the measurement of thermodynamic work of adhesion.

The work of adhesion now can be expressed with the Young-Dupre equation:

$$
\gamma_{A}=\gamma_{f}+\gamma_{S}-\gamma_{f S}=\gamma_{f}(1+\cos \Theta)
$$


Material particles in thermal equilibrium can be obtained by the sessile drop method [LL94] or by annealing [LCE98]. When the specific surface energy of the film $\gamma_{f}$ is known at a given temperature $T_{0}$, at any temperature $T$ it is [VMG02]:

$$
\gamma_{f}(T) \approx \gamma_{f}\left(T_{0}\right)+\left(T-T_{0}\right)\left(\frac{\partial \gamma_{f}}{\partial T}\right)_{T=T_{0}} .
$$

The solution of Eqs. (1.3) and (1.4) at the annealing temperature gives the value of the thermodynamic adhesion energy. The annealing has to be performed in vacuum in order to avoid oxidation. Other problems referred to this technique are too high required annealing temperatures for many materials (e.g. metals on polymer substrates), adsorption and diffusion bonded materials during annealing and a poor reproducibility of the results.

Contact angle measurements can be obtained from SEM or AFM image analysis [LCE98]. The true work of adhesion is a constant for a given film-substrate-system, typically ranging 0.5-3 $\mathrm{J} / \mathrm{m}^{2}$. Applying the contact angle method, for metals on ceramics values in the order of $0.5-2$ $\mathrm{J} / \mathrm{m}^{2}$ are reported [VMG02], while Reimanis et al. [RDR90] and Lipkin et al. [LCE98] determined the thermodynamic work of adhesion of gold on sapphire to be $0.5-0.9 \mathrm{~J} / \mathrm{m}^{2}$. Furuya and co-workers calculated the adhesive energy of $\mathrm{Cu} / \mathrm{SiO}_{2}$ as $0.8 \mathrm{~J} / \mathrm{m}^{2}$.

Most test methods determine the work of adhesion by delaminating thin films from the substrate. During detachment from the substrate, the thin film and/or the substrate usually experience plastic deformation. Therefore, it is difficult to extract the true adhesive energy from the measured total energy. What is measured in this case is the practical work of adhesion:

$$
W_{A d, P}=G=W_{A}+U_{\text {plastic }}
$$

where $U_{\text {plastic }}$ is the energy stored in plastic deformation of the film and the substrate. The strain energy release rate or the crack driving force, $G$, is used in fracture mechanics and applied as a measure of the practical work of adhesion. The practical work of adhesion takes into account the thermodynamic work of the film to separate and the energies dissipated by the film and substrate during separation through plastic deformation. The definition of the thermodynamic work of adhesion requires that the system is in the thermodynamic equilibrium and that the process of delamination is reversible. In practice, however, delamination proceeds via crack propagation, which is indeed an irreversible process. Only if the energy dissipation from the system is 
negligible, the interfacial strain energy release rate is equal to the thermodynamic work of adhesion.

The amount of plastic deformation at the crack tip and the practical work of adhesion are increasing with film thickness [VMG02]. Therefore, it can be assumed that thinner films give more precise results for the determination of the thermodynamic adhesion energy.

In the following, some different adhesion tests will be described and annotated in more detail to give an overview and show the application limits of such methods. There are more than 350 different methods of adhesion tests listed in literature [M95]. The choice of the desired test depends on the investigated material and the sample geometry. For sputtered metallic films on ceramic substrates often the scratch test [VKG93] is used and for metallic films on polymer substrates the peel test [YH98] is applied.

In the case of adhesion studies by using of the SFM (scanning force microscope) technique a cantilever tip, normally used in surface force microscopy, is coated with the interesting metal and its interaction with a surface of a substrate is studied. The theoretical background for this method has been established by Johnson, Kendall and Roberts [JKR71], and by Derjaugin, Mullet and Toporov [DMT75], respectively. Johnson et al. developed a theory (JKR theory) providing the values of the work of adhesion between two bodies with a certain contact area. This theory is applicable for tips with large curvature radius (most likely to macroscopic bodies) and small stiffness. Derjaugin et al. formulated a theory (DMT theory), which is applicable for tips with small curvature radius and high stiffness. These two theories were developed further by Maugis. His model is a more common approach and can be applied to any materials system with both high and low adhesion [M92].

The mechanical adhesion measurement can be non-destructive or destructive. An example for non-destructive techniques is an ultrasonic characterization of interfacial adhesive bonds [WQL06]. The destructive mechanical tests can only provide information about the energy necessary to deform and break the interface of the tested system. Such methods applied to these days cannot evaluate adhesion energies with high accuracy. More complex mathematical models and a deeper physical understanding of the nature of bonding are needed.

Adhesion tests can be also classified by the kind of stresses generated in the interfacial region. There are two classes of tests [SH88], namely tensile (e.g. acceleration, shock wave test) and shear tests (e.g. peel, tape, scratch tests). Nevertheless, all these tests are qualitative because of the difficulties in separating the adhesion energy from the total energy of film detachment.

In pull-off test a normal tensile force is applied to the coating using an element, which was before glued or soldered to the coating. The difficulties here are to avoid diffusion of the bonding 
agent through the coating that would result in a modification of the properties of the interface, and to produce uniform tensile stresses in the tested area.

In acceleration tests there are two possibilities to generate tensile stresses in the coating: Ultra centrifugal and ultrasonic methods [SH88]. In the first method a coated cylinder is levitated electromagnetically and rotated with an increasing speed until detachment of the coating from the substrate. In the second acceleration test the ultrasonic vibrations in the sample using an electromagnetic or piezoelectric transformer are generated. In both tests the tensile stresses produced in the coating directly depend on the mass of the coating and therefore on its thickness. Therefore, the adhesion testing of films thinner than $10 \mu \mathrm{m}$ requires extremely large acceleration.

In shock-wave tests a compressive shock wave perpendicularly to the coating is produced in the sample (e.g. by a pulsed laser). The adhesion is characterized by the energy density per unit area of the impulse responsible for detaching the coating.

The basic idea of the peel test is that the tested sample is held in a holder which moves horizontally with the same velocity as coating that is pulled from the substrate vertically, maintaining a $90^{\circ}$ angle between the peel and the substrate. The measured peel strength consists of two components: The force required to plastically deform the film and the decohesive force at the interface. It is difficult to extract the adhesion energy from the peel strength. Another problem is a high dependency of the measured energies from the peel force and the peel rate [K71].

In scratch tests a stepwise or continuously loaded spherical scratching point is drawn across the coated sample. The scratching point produces an increasing elastoplastic deformation of the film-substrate-system until damage occurs in the surface region. The minimum load at which an adhesive failure occurs is representative of the coating adhesion.

In the blister test, gas flow pressure is applied to a portion of the film suspended over a hole in the substrate until the film debonds or "blisters" from the substrate. The work of adhesion is then calculated from the measured pressure at which peel/blister initiates. A basic problem concerning this method is the possibility that very thin or very adhesive films might break before blistering occurs. Another problem refers the geometrical constraint that cracks do not propagate along the perimeter of the blister, making the interpretation of the results difficult.

A theory for the adhesion energy measurement by the determination of the profile of delaminated area was proposed by Gille and Rau [GR84]. They have shown that the adhesion energy between film and substrate can be determined from the geometric parameters of the wrinkle, such as the height and the width of the wrinkle and from the elastic properties of the film, expressed by Poisson ratio and elastic modulus of the film. However, the width and height of a straight-sided buckle is not easily determined experimentally from several reasons. First, it is 
difficult to find a straight-sided buckle with the ideal cross-section in a common sample and second, typical size of buckles are in the micrometer range, where optical equipments are at their lower limit and scanning probe microscopy techniques are at their upper limit. Additionally, plastic deformations of the wrinkles can occur, affecting the adhesion energy calculation from the geometry of wrinkles. The adhesion energy depends on the fourth power of the height-towidth ratio in the Gille and Rau approximation. Therefore, this ratio sensitively influences the calculated adhesion energy.

Some other known techniques for quantitatively adhesion energy measurement, such as an extended microbridge test, probe test, a modified peel test and microindentation tests have manifold limitations or require complicated techniques and sample geometries in application.

Summarizing this short overview of adhesion tests it can be pointed out that the search for an adhesion test whose performance is close to an ideal adhesion test is not yet completed. The ideal test should be non-destructive, automated, easy to perform, easy to interpret, reproducible and quantitative. In the following chapter a new method is presented which gives an opportunity to measure the adhesion energy close to the ideal way. This test is characterised with minimal destruction of the interface bond during the measurement by film buckling, easy to perform, based on a simple mathematical model and gives the quantitative results of adhesion energy.

\subsection{Controlled buckling method for adhesion energy calculation}

\subsubsection{The idea of this method}

The idea of this method is shown in Fig. 1.2. A high compressive stress can be introduced in a metal film by charging with solute atoms. The increase of elastic energy leads to film detachment by overcoming the adhesion energy. The critical stress for film delamination provides information about the adhesion energy of the film to the substrate.

a)

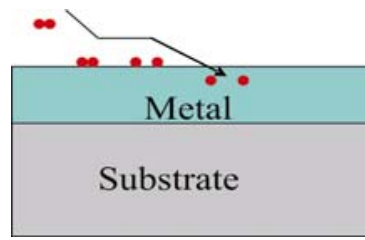

b)

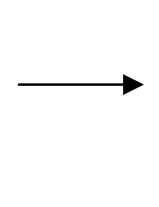

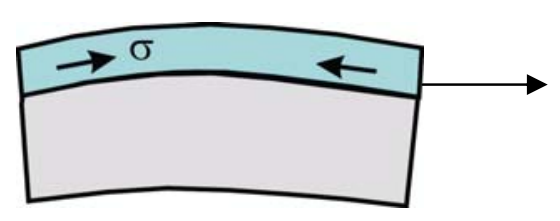

c)

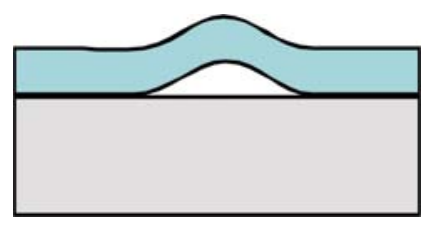

Figure 1.2: The principle of controlled buckling method: a) absorption of solute atoms in to the metal film, b) stress increase in the metal film, c) film detachment via buckle formation. 
In this work controlled hydrogen loading of metal films was applied, leading to stress formation and delamination of the film from the substrate. Why do we use hydrogen as a solute? It is known that hydrogen diffuses much faster than any other atoms in solids, because small, light weight atoms diffuse faster than larger, more massive atoms. In Fig. 1.3 some examples of diffusion coefficients of some elements in $\mathrm{Nb}$ and $\alpha-\mathrm{Fe}$ are shown.

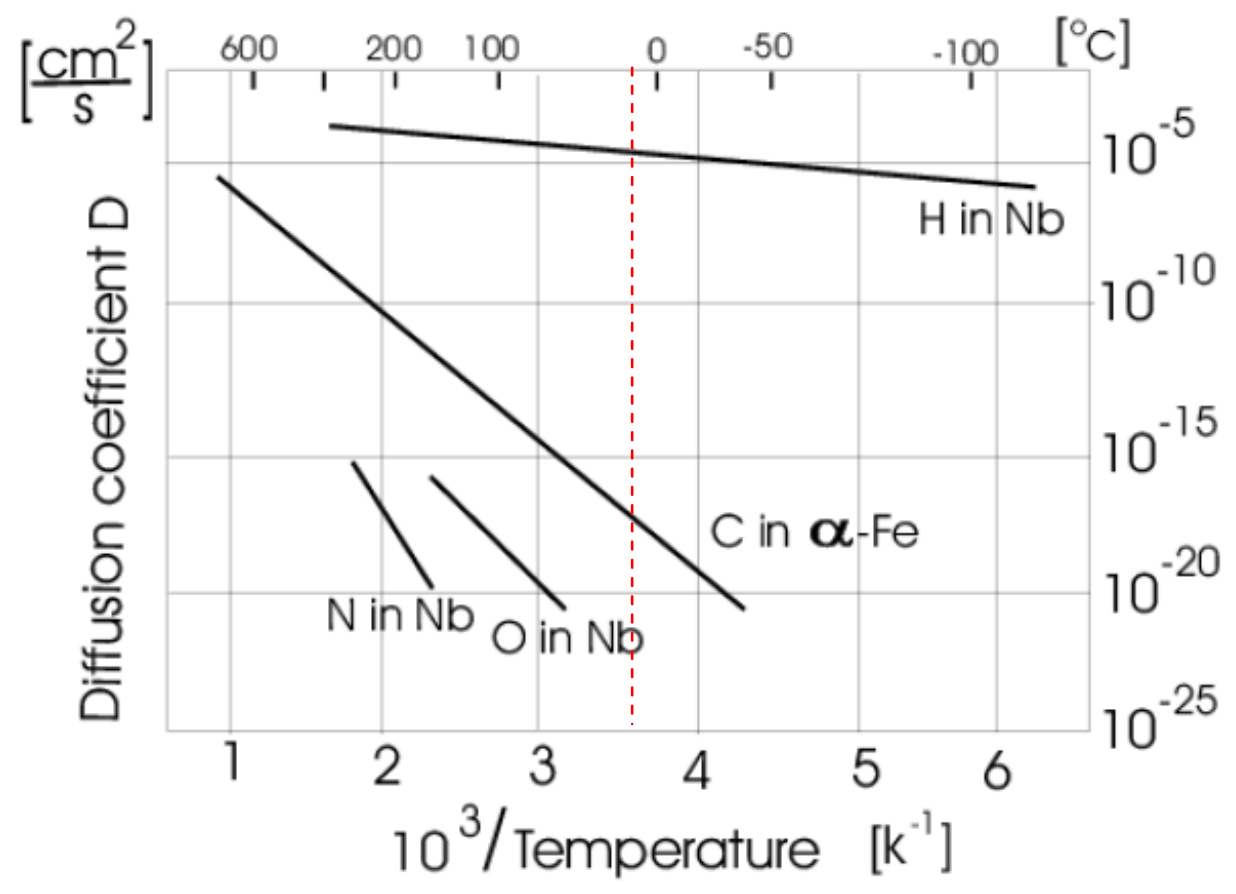

Figure 1.3: Diffusion coefficients of $H, N$, and $O$ in $N b$ and $C$ in a $\alpha-F e$ [AV78]. The diffusion coefficient of $\mathrm{H}$ in $\mathrm{Nb}$ is much larger than that of other gases in a wide temperature range. Room temperature is shown with the dashed line.

Diffusion coefficients of $\mathrm{H}$ in $\mathrm{Nb}$ at room temperature and in the wide temperature range are much larger than the diffusion coefficients of other gases in $\mathrm{Nb}$.

In this work the $\mathrm{Nb}$ has been applied for the hydrogen absorption. The choice of $\mathrm{Nb}$ resulted from its large capability of absorbing hydrogen. For example, the solubility of hydrogen in niobium within the low concentration range at room temperature is $2.8 \cdot 10^{5}$ times larger than that in palladium at the same hydrogen pressure [W82]. The phase diagram of hydrogen in bulk $\mathrm{Nb}$ crystals is shown in Fig. 1.4, where $\mathrm{c}_{\mathrm{H} / \mathrm{Nb}}$ is hydrogen concentration. Above room temperature depending on the hydrogen concentration three phases appear: $\alpha, \alpha^{\prime}$, and $\beta$, where the $\alpha$ and $\alpha^{\prime}$ phases are disordered solutions of low and high hydrogen concentrations, respectively.

The solid solution $\alpha-\mathrm{Nb}-\mathrm{H}$-phase has a cubic body-centred (bcc) crystal structure. It solves $\mathrm{H}$ up to a concentration $\mathrm{c}_{\mathrm{H}}=0.06 \mathrm{H} / \mathrm{Nb}$ at $300 \mathrm{~K}$, above which the formation of the $\mathrm{Nb}-\mathrm{H}-\mathrm{Hydride}$ occurs. Depending on temperature and concentration two different hydrides are present, $\alpha^{\prime}$ and $\beta$ 
phase. In the $\alpha$ and $\alpha^{\prime}$ phases hydrogen atoms are randomly distributed in the host lattice, and the $\mathrm{Nb}$ crystal retains its cubic symmetry, while the lattice constants are different. Because of the similarity to gas and liquid phase transition, $\alpha$ and $\alpha^{\prime}$ are called lattice gas and lattice liquid phase. The $\alpha^{\prime}-\mathrm{Nb}-\mathrm{H}-$ phase is thermodynamically stable for $\mathrm{T}>361 \mathrm{~K}$. For hydrogen in bulk Nb, the critical temperature of the $\alpha-\alpha^{\prime}$ phase transition is $444 \mathrm{~K}$, and the related critical hydrogen concentration is $\mathrm{c}_{\mathrm{H}}=0.31 \mathrm{H} / \mathrm{Nb}[\mathrm{H} 96]$.

With increasing $\mathrm{H}$-concentration above $0.06 \mathrm{H} / \mathrm{Nb}$ and at room temperature the ordered $\beta-\mathrm{Nb}-\mathrm{H}$ phase is formed. The crystal lattice of the $\beta-\mathrm{Nb}-\mathrm{H}$ phase is face centred orthorhombic. Compared to the $\alpha$-phase the lattice is stretched and orthogonally deformed. The deformation is small, $\mathrm{c} / \mathrm{a}=1.005$ [WR70]. The $\beta-\mathrm{Nb}-\mathrm{H}$ phase exists from $\mathrm{c}_{\mathrm{H}}=0.70 \mathrm{H} / \mathrm{Nb}$ up to $1.0 \mathrm{H} / \mathrm{Nb}$. The $(\alpha, \beta)-$ miscibility gap at room temperature extends from $\mathrm{c}_{\mathrm{H}}=0.06 \mathrm{H} / \mathrm{Nb}$ to $\mathrm{c}_{\mathrm{H}}=0.72 \mathrm{H} / \mathrm{Nb}$. The $\delta-\mathrm{Nb}-$ $\mathrm{H}$ phase is formed up to $\mathrm{c}_{\mathrm{H}}=1.1 \mathrm{H} / \mathrm{Nb}$ [WR70].

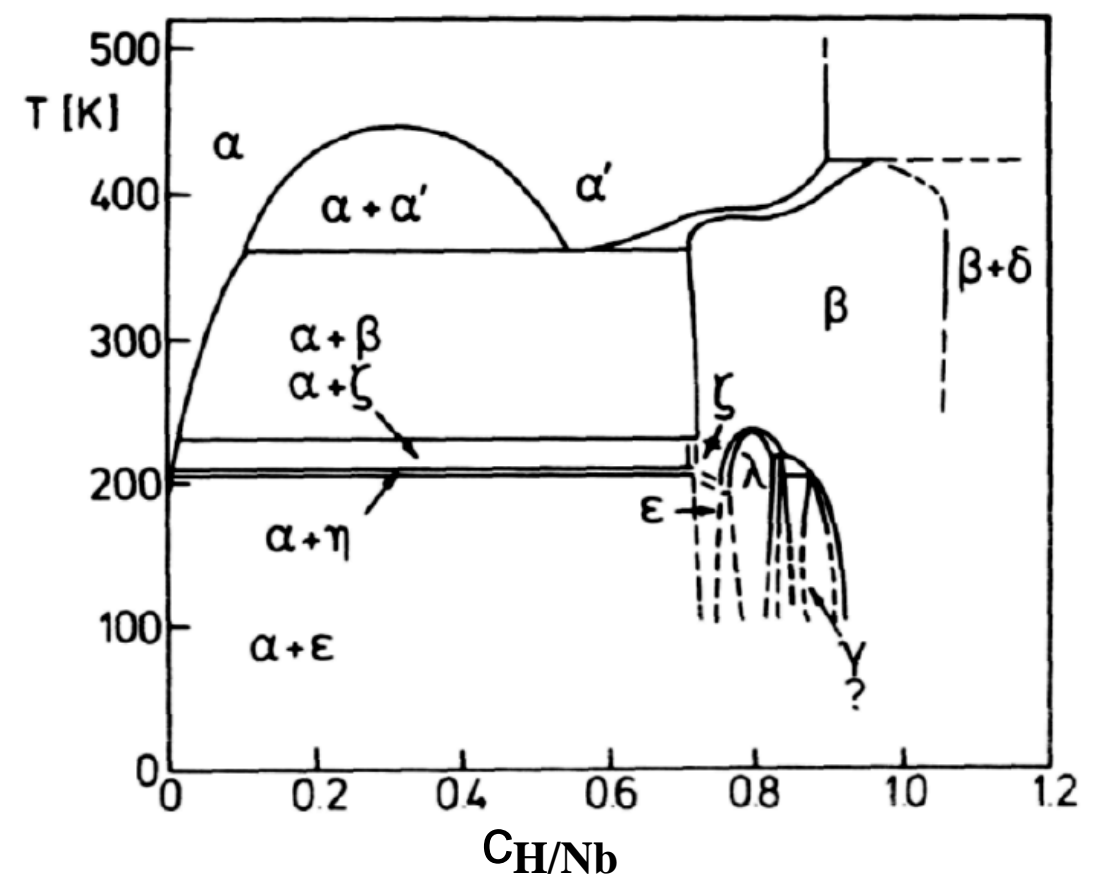

Figure 1.4: The phase diagram of hydrogen in bulk Nb crystals [SchW78].

Compressive stresses are built up in a film on a substrate by absorbing solute atoms or molecules in the film. In a metal film not attached to a substrate, due to hydrogen absorption isotropic volume expansion $\Delta \mathrm{V} / \mathrm{V}$ occur. The volume expansion depends linearly on the hydrogen concentration in the whole concentration range [SchW78]. 


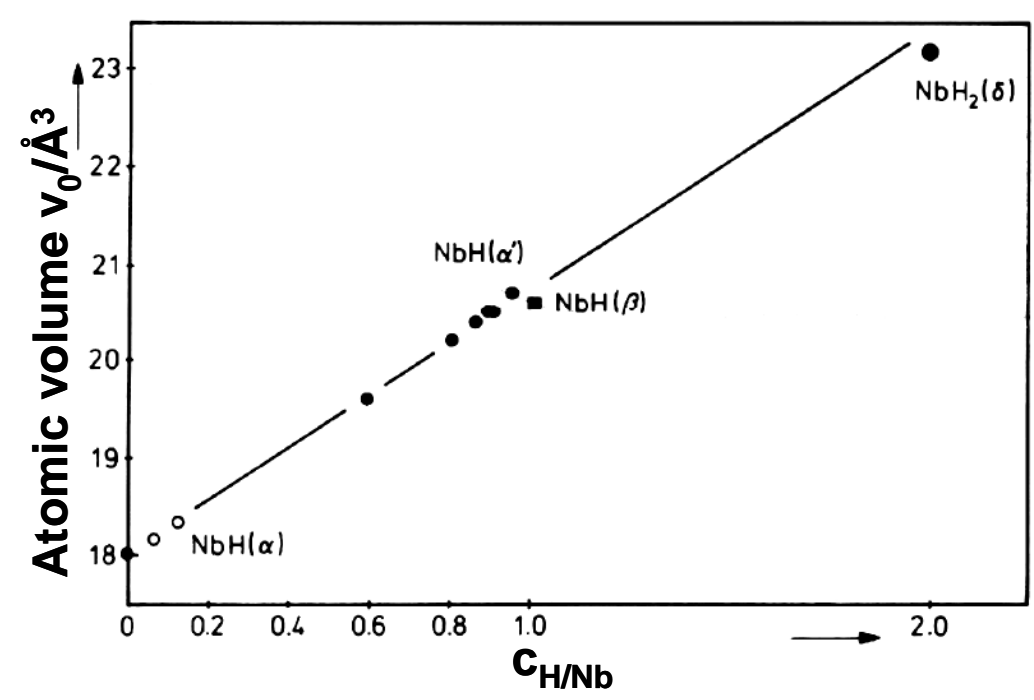

Figure 1.5: Increasing of $\mathrm{Nb}$ volume vs. hydrogen concentration [SchW78].

In Fig. 1.5 the linear increase of the $\mathrm{Nb}$ bulk volume with increasing hydrogen concentration is shown. For the volume expansion in the $\alpha-\mathrm{Nb}-\mathrm{H}$ phase Peisl [P78] gives

$$
\frac{\Delta V}{V_{0}}=\frac{\Delta v}{\Omega} \cdot c_{H}=0.174 \cdot c_{H}
$$

where the volume increase per hydrogen atom is $\Delta v=3.13 \AA^{3}, \Omega$ the average volume of a one $\mathrm{Nb}$ atom and $\mathrm{c}_{\mathrm{H}}$ the hydrogen concentration $(\mathrm{H} / \mathrm{Nb})$. For the $\gamma-\mathrm{NbH}_{2}$ phase $\Delta v$ is $2.67 \AA^{3}$. For a cubic crystal with a lattice constant $a$ and a random occupation of the interstitial sites by H-atoms it is

$$
\frac{a_{c_{H}}^{3}-a_{0}^{3}}{a_{0}^{3}}=\frac{\Delta v}{\Omega} \cdot c_{H} \approx 3 \frac{\Delta a}{a} .
$$

Therefore the lattice expansion for bulk Nb can be calculated with Eq. (1.6) as

$$
\varepsilon_{0, N b}=\left(\frac{\Delta d}{d_{0}}\right)_{b u l k} \approx \frac{1}{3} \frac{\Delta V}{V_{0}}=0.058 \cdot c_{H}
$$

For a well-adhering film on a rigid substrate lattice expansion is prevented in-plane, while expansion is possible only in the out of plane direction. Therefore, within the plane of the film compressive stresses up to several $\mathrm{GPa}$ are induced. For hydrogen in metal films the corresponding strains and stresses can be calculated as [B98]

$$
\sigma=\frac{-E}{3(1-v)}\left(\frac{\Delta V}{V_{0}}\right)=\frac{-E v_{p} c}{3(1-v)},
$$

where $v_{p}$ is the partial molar volume of the solute and $c$ its concentration. Thus, compressive stress is increasing linearly with increasing solute concentration $c$. 
For an isotropic $\mathrm{Nb}$-film, clamped to a rigid substrate, the expansion in direction normal to the film layer is [Lau98]

$$
\varepsilon_{3}^{\prime}=0.131 \cdot c_{H}
$$

while the in-plane stress increases as

$$
\sigma_{x x}=\sigma_{y y}=-9.7 G P a \cdot c_{H}
$$

Thus, by hydrogen loading it is possible to achieve high in-plane compressive stress up to several $\mathrm{GPa}$ in strongly bond $\mathrm{Nb}$ thin films, linearly depending on hydrogen concentrations for low values of $\mathrm{c}_{\mathrm{H}}$. Laudahn has observed stresses up to $-9.7 \mathrm{GPa} / \mathrm{c}_{H}$ for laser deposed $\mathrm{Nb}$-films. In this work this effect is used in controlled hydrogen induced delamination method and applied for the evaluation of the adhesion energy between thin films and substrates.

In order to enable hydrogen absorption by the niobium film, a thin (10 to $20 \mathrm{~nm}$ ) palladium film can be deposited onto the niobium film. Thereby palladium films with the thickness of 10 $\mathrm{nm}$ are closed [Wag05]. This top palladium layer prevents oxidation of the niobium film and acts as catalyst for hydrogen absorption. Since hydrogen solubility of niobium is $2.8 \cdot 10^{5}$ times larger than that of palladium, dissolved hydrogen atoms diffuse into the niobium layer after dissociation of hydrogen molecules at the palladium surface. Therefore, hydrogen is preferentially absorbed in the Nb-layer and the hydrogen content within the Pd-layer can be neglected, and the Nb-layer is regarded as active layer generating and transferring mechanical stress in the systems $\mathrm{Nb} / \mathrm{Pd}$ or $\mathrm{Me} / \mathrm{Nb} / \mathrm{Pd}$ (with $\mathrm{Me}=$ metal).

To summarize, the idea of the controlled buckling method is to apply in-plane stresses in a controlled way using the electrochemical hydrogen loading technique which will be explained in the next chapter. Hydrogen loading of a Pd-covered $\mathrm{Nb}$ film generates high in-plane stress up to several GPa. This stress increases linearly at low hydrogen concentration (i.e.: in the elastic region). At the critical hydrogen concentration (critical stress) the buckling occurs. Thereby elastic stress in the metal film sample is reduced. The critical stress for buckling can be measured by determining the curvature of the substrate.

\subsubsection{Controlled hydrogen absorption, electrochemical method}

For the absorption of a definite amount of hydrogen in a metal film an electrochemical hydrogen loading method was used [KL80]. Applying Faradays law, this method gives the 
possibility to load metal films with defined amounts of hydrogen. The number of hydrogen atoms, discharged at the metal surface in a vicinity of electrolyte, thereby directly follows from the applied current between the metal and a counter electrode [KSSch82]. The loading is quick by using of a simple setup and the evaluation procedure is not difficult.

The absorption of hydrogen happens in two steps:

$$
\begin{aligned}
& \mathrm{H}_{3} \mathrm{O}^{+}+e^{-} \leftrightarrow H_{a d}+H_{2} \mathrm{O} \\
& \text { and } H_{a d} \leftrightarrow H_{a b}
\end{aligned}
$$

$\mathrm{H}_{3} \mathrm{O}^{+}$is a result of protolysation of water (a proton is released from the acid and combined with a water molecule). The two steps consist of the adsorption of hydrogen on the metal surface followed by a chemical absorption into the subsurface metal layer [B65]. The kinetics of reaction (1.12) depends on the characteristics of the electrode, i.e. on the surface layer of the sample. In case of Pd the charge transfer described by (1.12) runs particularly fast [Not95]. For the charging procedure, the samples were contacted by Teflon isolated cables and conductive silver-paint. Afterwards the contact point was electrically isolated by applying a two-components-glue. During the electro-chemical loading the sample has a negative potential (cathode) and the counter electrode a positive potential (anode). The following reactions take place at the sample surface:

$$
\mathrm{Pd}+\mathrm{H}_{3} \mathrm{O}^{+}+e^{-} \rightarrow \mathrm{Pd}-\mathrm{H}+\mathrm{H}_{2} \mathrm{O}
$$

and at the counter electrode:

$$
\mathrm{Pt}+6 \mathrm{H}_{2} \mathrm{O} \rightarrow \mathrm{Pt}+\mathrm{O}_{2}+4 e^{-}+4 \mathrm{H}_{3} \mathrm{O}^{+} .
$$

Due to the lower enthalpy of $\mathrm{NbH}_{\mathrm{x}}$ formation, hydrogen previously solved in the $\mathrm{Pd}$ preferentially diffuses to the Nb-layer, until a concentration of about $c_{H}=1$ is reached there. The quantity of hydrogen absorbed by the sample can be determined by means of Faradays law:

$$
n_{H}=\frac{I \cdot t}{F}
$$

where $Q=I t$ is the transferred charge, $F$ is Faradays constant and $n_{H}$ the quantity of the hydrogen in moles. The H-concentration $\mathrm{c}_{\mathrm{H}}$ in the niobium layer results from the volume $V$ of the layer, the molar volume $V_{m}$ of niobium and the quantity of hydrogen determined according to Faraday law:

$$
c_{H}=\frac{n_{H}}{n_{N b}}=\frac{I \cdot t}{F} \cdot \frac{V_{m, N b}}{V} .
$$




\subsubsection{Optical microscopy studies and stress measurement}

As described in chapter 1.2.1 the idea of the controlled film delamination method for adhesion energy measurements consists of the measurement of the critical stress or hydrogen concentration, at which delamination of a metal film from the substrate occurs.

The stress calculation is carried out by applying Stoney's formula, derived in 1909 [Sto09],

$$
\sigma_{f}=\frac{E_{S} t_{S}^{2}}{6\left(1-v_{S}\right) t_{f} R}
$$

where $E_{S}$ is the Young's modulus, $R$ the radius of curvature of the substrate and $v_{s}$ is Poisson's ratio of the substrate. This formula simply relates an induced substrate curvature to the film stress. The radius of curvature has been derived by measuring the vertical displacement $z$ at the end of a sample by using an inductive displacement gauge. There are some mechanical assumptions and geometrical limitations necessary for the application of this equation. This expression is defined supposing an in-plane stress isotropy of the deposited film. The deformation has to be elastic for film and substrate. The thickness of the film has to be uniform along the sample and its adhesion is assumed to be perfect. Further, the expression is only valid for a film thickness much smaller than the substrate one. Because of the thin-film approximation for the coating stress, a ratio of $1 / 20$ is acceptable [K00]. The length-to-width ratio has to be as large as possible; in this case the influence of transverse deformation can be neglected. Sander [San99] has shown that the ratio length/width should to be larger then 2. In this work samples with a ratio of 4 were used.

In the following, the relationship between the curvature radius of a sample and the bending displacement at the end of a sample will be derived. Fig. 1.6 shows a deflected sample $B K$.

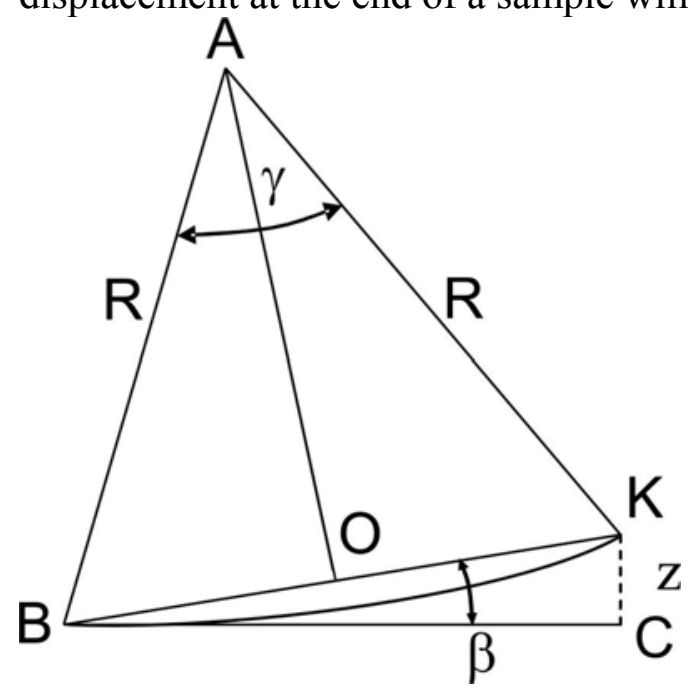

Figure 1.6: Schematic picture for the curvature radius derivation. 
With the assumption that $\stackrel{\cap}{B K}=B K=L$ and $\angle B C K=90^{\circ}$, where $L$ is the length of the sample, it follows

$$
z=L^{*} \sin \beta
$$

Since $A O \perp B K$ and $B C \perp A B$,

$$
\beta=\frac{\gamma}{2}
$$

From (1.17) and (1.18) the vertical displacement during the sample bending is

$$
z=L * \operatorname{Sin} \frac{\gamma}{2}
$$

For small angles $\sin \gamma \approx \gamma$. The angle $\gamma$ can be derived as $\gamma=\frac{L}{R}$. So, formula (1.19) can be rewritten as

$$
z=\frac{L^{2}}{2 R}
$$

And, finally, the curvature radius of the sample is determined as

$$
R=\frac{L^{2}}{2 z}
$$

This means that due to the assumptions there are only two parameters determining the curvature radius: the length of the sample and the vertical displacement of the sample at this length during the bending process.

The calculated radius of curvature $R$ was inserted in Eq. (1.16) together with the elastic constants of the substrate, yielding the compressive stress in the metal.

\subsubsection{Mathematical description of buckling}

How is the delamination (buckling) of thin films on substrates mathematically described? Depending on small variations in film stress, thickness and interface adhesion, buckle delamination occurs locally and then propagates across the film in one of two basic morphologymodes: telephone cord buckling or straight-sided (Euler) buckling. Further, there is the varicose mode, which is a transition mode between the two basic modes (Fig. 1.7). 

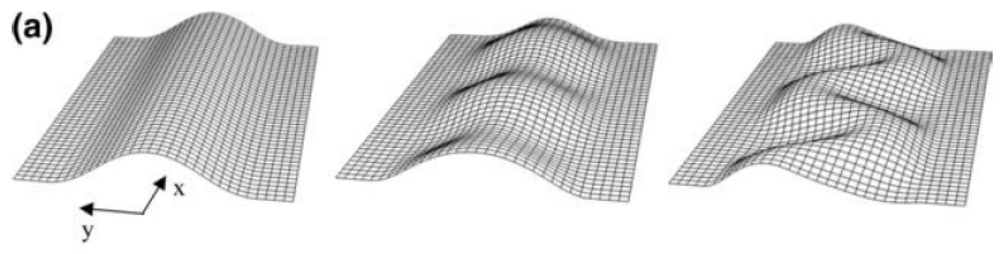

(b)

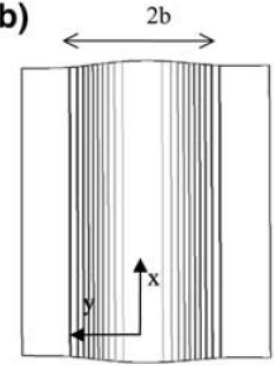

Euler

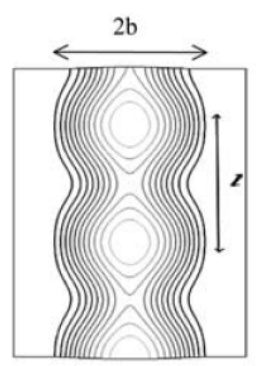

Varicose

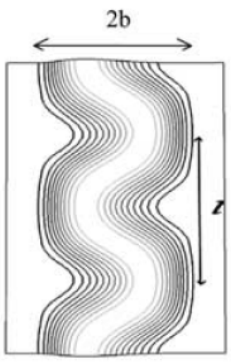

Telephone cord

Figure 1.7: Geometry (a) and finite element mesh (b) for buckling analysis of a film clamped along its edges $a t y= \pm b$ to a rigid substrate (this image is taken from [M04]).

\section{Free plate buckling}

The buckling behaviour of a compressed film that is detached from a substrate over a strip of width $2 b$ is usually modelled as a plate of the same width that is fully clamped along its ends. Young's modulus of the substrate in this model is assumed to be not less than about one fifth of that of the film [YH02]. When the substrate has a very low modulus compared to the modulus of the film, deformation of the substrate along the edge of the detached region becomes important. The onset of buckling mode will be described by the Euler mode as the first stage of the buckle formation. The critical compressive biaxial stress at the onset of buckling, $\sigma_{E u}$, is [HS92, LL86]

$$
\sigma_{E u}=\frac{\pi^{2}}{3} \cdot \frac{E}{1-v^{2}}\left(\frac{d}{l}\right)^{2}
$$

where $E$ and $v$ are Young's modulus and Poisson's ratio of the film, and $d$ is its thickness. For a given film stress, $\sigma_{0}$, it is useful to define the width $l_{0}$ of buckles at the onset of buckling, which is given by Eq. (1.22) with $\sigma_{E u}=\sigma_{0}$ :

$$
l_{0}=\frac{\pi \cdot d}{\sqrt{3}} \sqrt{\frac{E}{\left(1-v^{2}\right) \sigma_{0}}},
$$

where $\sigma_{0}$ is the biaxial compressive stress in the unbuckled plate.

It follows that

$$
\frac{\sigma_{0}}{\sigma_{E u}}=\left(\frac{l}{l_{0}}\right)^{2} .
$$


Thus, the stress is directly related to the width of the buckles. The sinusoidal shape of a buckle is described by the normal displacement of the buckling mode [M04]

$$
w=\frac{\xi \cdot d}{2}\left(1+\cos \left(\frac{2 \pi \cdot y}{l}\right)\right),
$$

where $\xi$ is the dimensionless buckling amplitude which depends on the ratio of stresses in the unbuckled film to the value at the onset of buckling,

$$
\xi \equiv\left(\frac{w_{\max }}{h}\right)=\sqrt{\frac{4}{3}\left(\frac{\sigma_{0}}{\sigma_{E u}}-1\right)}
$$

The average energy release rate during buckling is the difference between the energy per area in the plate, $W_{0}$, prior buckling and the average energy per unit area in the buckled state $\bar{W}$, i.e. $\bar{G}=W_{0}-\bar{W}$. The energy per area stored in the film in the unbuckled state by equibiaxial compression is

$$
W_{0}=\frac{1-v}{E} \sigma^{2} d,
$$

while the average energy per area in the buckled state is calculated using $(1.22,1.25,1.26)$ to be [HS92]

$$
\bar{W}=W_{0}\left(1-\left(\frac{1+v}{2}\right)\left(1-\frac{\sigma_{E u}}{\sigma_{0}}\right)^{2}\right)
$$

The energy release rate is the difference between the energy per area in the unbuckled film and in the buckle region $W_{0}-\bar{W}$. For the Euler mode it is [HS92]

$$
\bar{G}=G_{0}\left(1-\frac{\sigma_{E u}}{\sigma_{0}}\right)^{2},
$$

where $G_{0}$ is the available energy per area stored in the unbuckled film subject to release under plane strain conditions (with no strain change parallel to the crack front and with the displacements of all points in the film during delamination normally to the substrate-film interface):

$$
G_{0}=\frac{1+v}{2} W_{0}=\frac{\left(1-v^{2}\right) \sigma_{0}^{2} d}{2 E}
$$

\section{Adhered plate buckling}

In the following the mathematical modelling of the buckling process will be presented, taking advantage of two different expressions for the elastic energy of the film, which is required for buckling: in model 1 the elastic energy will be calculated by Eq. (1.30). In this case stress release 
during buckle formation is uniaxial. In model 2 the elastic energy for buckling is calculated using Eq. (1.27) for biaxial stress release.

The controlled buckling method is based on a simple model connecting the onset of buckling with the adhesion energy. The buckling process of the film occurs when the stored elastic strain energy overcomes the adhesion energy. A schematic figure after film buckling by uniaxial stress release is shown in Fig. 1.8, assuming Euler mode buckling. From Eq. (1.30) the elastic energy per volume $V=d \cdot l \cdot L$ is calculated as

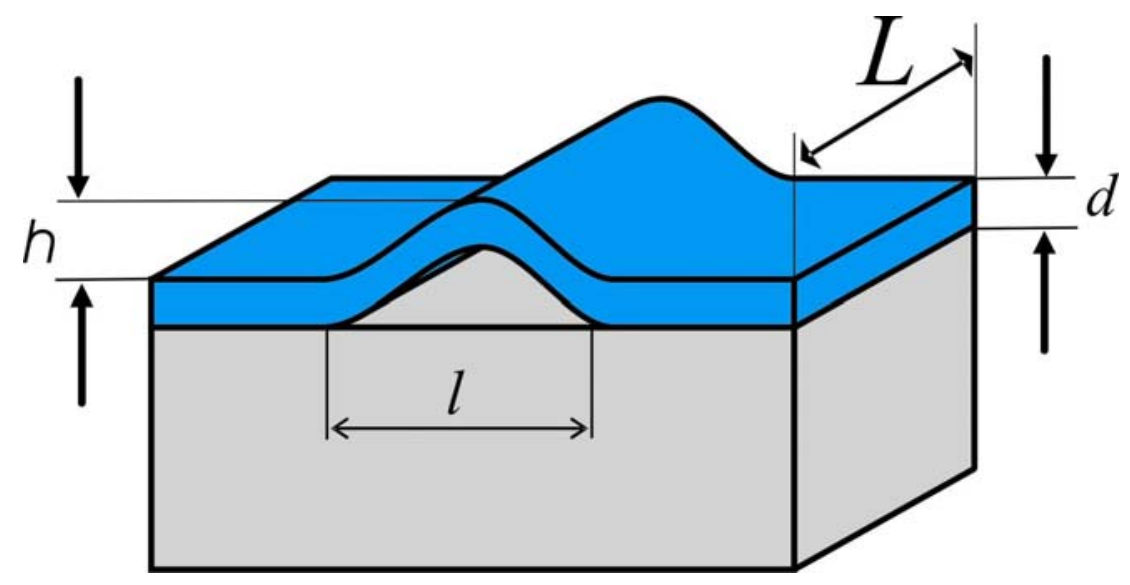

Figure 1.8: Schematic drawing of the cross-section of a buckle.

$$
W_{e l}=\frac{E}{2\left(1-v^{2}\right)}\left(\varepsilon_{i n}+\varepsilon\right)^{2} \cdot d \cdot l \cdot L=\frac{\left(\sigma_{i n}+\sigma\right)^{2} d \cdot l \cdot L\left(1-v^{2}\right)}{2 E}
$$

where $\varepsilon$ is the strain and $\sigma$ the stress in the film induced by a solute molecule or atom, whereas $\varepsilon_{\text {in }}$ and $\sigma_{\text {in }}$ are the initial values present after film preparation. The width of the buckled region is $l=2 b$, its length $L$, and $d$ is the film thickness. Eq. (1.31) is an approximation as it assumes that the buckled region of the film is free of stresses. The adhesion work $W_{a d}$ for the detached region of the film is given by

$$
W_{a d}=\gamma \cdot l \cdot L
$$

where $\gamma$ is the adhesion energy per unit area. It is equivalent to the thermodynamical work of adhesion in Eq. (1.3).

If the released elastic energy is equal or larger than the energy of adhesion, buckling is energetically possible and Equations (1.31) and (1.32) result in a corresponding critical stress 


$$
\left|\sigma_{c r i t}\right|= \pm\left|\sigma_{i n}\right|+\sqrt{\frac{2 \gamma E}{d\left(1-v^{2}\right)}},
$$

where the positive sign corresponds to tensile initial (intrinsic) stresses, i.e., $\sigma_{\text {in }}>0$ and the negative sign to compressive initial stresses.

Assuming a linear strain of the Nb-layer during H-loading, $\varepsilon=\alpha \cdot c_{H}$, where $\alpha$ is the material expansion factor upon hydrogen loading, $\alpha=0.058$ for $\mathrm{Nb}$ [P78], the critical hydrogen concentration for the delamination is

$$
c_{H, c r i t}=\frac{ \pm\left|\varepsilon_{i n}\right|}{\alpha}+\frac{1}{\alpha} \sqrt{\frac{2 \gamma\left(1-v^{2}\right)}{E d}} .
$$

For the refined mathematical model [PNPK04] including remaining stresses a calculation of the remaining stresses and the corresponding remaining elastic energy $W_{r e}$ per buckle length gives for equilibrium of forces [GR84]:

$$
W_{r e}=\frac{\pi^{2} E d l L}{6\left(1-v^{2}\right)}\left(\frac{d}{l}\right)^{2} \mid \varepsilon_{c r i t}+\varepsilon_{i n}\left\{2+\frac{\pi^{2}}{3\left(\varepsilon_{\text {crit }}+\varepsilon_{\text {in }}\right)}\left(\frac{d}{l}\right)^{2}\right\},
$$

where $\varepsilon_{c r}$ is the critical elastic strain induced by solutes, which leads to buckling. Then the height of the buckle can be written as

$$
\frac{h}{l}=\sqrt{\frac{4\left|\varepsilon_{c r i t}+\varepsilon_{i n}\right|}{\pi^{2}}\left\{1+\frac{\pi^{2}}{3\left(\varepsilon_{c r i t}+\varepsilon_{i n}\right)}\left(\frac{d}{l}\right)^{2}\right\}}
$$

For the conditions of this study the second term in brackets, i.e. the contribution from the deflection to the remaining energy of the buckle [GR84], can be neglected. For buckling of a 100 $\mathrm{nm} \mathrm{Nb}$ film on PC the value in brackets is about 0.06. If this remaining energy is included in the energy balance for the energetically possibility of buckling, equations (1.31), (1.32) and (1.35) give

$$
\frac{\left(\sigma_{i n}+\sigma_{c r}\right)^{2} d\left(1-v^{2}\right)}{2 E}=\gamma+\frac{\pi^{2} d}{3}\left(\frac{d}{l}\right)^{2}\left|\sigma_{i n}+\sigma_{c r}\right| .
$$

In model 2 the elastic energy per volume $V$ in the film is calculated according to (1.27) for equibiaxial compression and stress release during buckling in the thin film

$$
W_{e l}=\frac{E}{1-v}\left(\varepsilon_{i n}+\varepsilon\right)^{2} \cdot d \cdot l \cdot L .
$$

By assuming that film delamination occurs when the elastic energy in the film segment equals the adhesion energy, the critical strain for buckling is 


$$
\varepsilon_{c r i t}= \pm\left|\varepsilon_{i n}\right|+\sqrt{\frac{\gamma(1-v)}{E d}} .
$$

And the critical hydrogen concentration then follows as

$$
c_{H, \text { crit }}=\frac{ \pm\left|\varepsilon_{\text {in }}\right|}{\alpha}+\frac{1}{\alpha} \sqrt{\frac{\gamma(1-v)}{E d}} .
$$

Therefore, Eq. (1.34) and 1.40) give different critical concentrations.

Using Hook's Law, $\sigma=\frac{E}{1-v} \cdot \varepsilon$, the critical stress for delamination can be calculated as

$$
\left|\sigma_{c r}\right|= \pm\left|\sigma_{i n}\right|+\sqrt{\frac{\gamma E}{d(1-v)}}
$$

The type of the compressive stress and its release in a thin film determines the shape of the buckles. Circular delaminations result in uniform in-plane stress release. Straight- sided buckles result in case of uniaxial stress release. IN this work straight sided buckles were mostly observed. Therefore, it was favoured the description with uniaxial stress release in the data discussions and eqs. (1.33) and (1.34) were applied for adhesion energy calculation using the controlled buckling method.

If the information about the adhesion energy is needed it is important to exceed the Euler stress $\sigma_{\mathrm{Eu}}$. In formula (1.22) it was shown that a plate under uniaxial compression becomes unstable, resulting in bending, if the critical stress is reached [LL86]:

Two limiting cases have to be considered for $\sigma_{c r}$ and $\sigma_{E u}$ (Eq. 1.22).

1. $\sigma_{E u}>\sigma_{c r}$, where buckle formation is determined by Euler's instability. The stress leading to buckling does not contain information about the adhesion energy.

2. $\sigma_{E u} \leq \sigma_{c r}$, where stresses leading to buckle formation do provide values for the adhesion energy, if the other parameters in Eqs. (1.41) or (1.33) are known.

The second case corresponds to the following inequality obtained from Eqs. (1.22) and (1.33):

$$
d^{5} \leq \frac{18\left(1-v^{2}\right) \gamma \cdot l^{4}}{\pi^{4} E}
$$

This condition can be fulfilled by reducing the thickness ( $l$ will decrease during a reduction of thickness $d$ ). For buckled $\mathrm{Pd} / \mathrm{Nb} / \mathrm{Pd}$ film (100 nm Nb film with thin Pd layers on the both sides) on PC the left side of Eq. (1.42) is two orders of magnitude smaller than the right part. This means that the measurement of stresses leading to buckling in such films gives an information about the adhesion energy of $\mathrm{Pd} / / \mathrm{PC}$ interface.

The procedure for evaluation of the adhesion energy is as follows. Metal films of two or more different film thicknesses are electrochemically loaded with hydrogen. At the same time the critical values for buckling (critical hydrogen concentrations or critical stress for buckling) are 
measured. The critical stress for buckling can be obtained from curvature measurements. In this case the end of the linear (elastic) increase of the mechanical stresses is measured. The critical hydrogen concentration can be simple measured by the surface observation to determinate the buckling appearance. A clear correlation of the endpoint of the linear range in stress curves and the critical H-concentration was verified in the experiment for metal films on PC. By hydrogen loading of $\mathrm{Nb}$ films on sapphire a decreasing stress was observed without film buckling. This reduction of stress occurs because of misfit dislocation generation and emission of extrinsic dislocation loops [PGB00, PLG00]. In this case the surface observation is necessary to obtain the correct critical values for buckling. In addition, buckling morphologies can be obtained and their development can be observed directly. The adhesion energy $\gamma$ between the film and the substrate can be determined by analyzing the critical hydrogen concentration or critical stress for films with different thickness $d$. A mathematical model was obtained by assuming film delamination when the elastic energy in the film segment equals the adhesion energy. Hydrogen loading of three different thicknesses of $\mathrm{Nb}$ layers can be used to determine the adhesion energy $\gamma$ according to Eq. 1.33 or 1.34 from the slope of straight line obtained from plotting of the critical stress or the critical hydrogen concentration versus the square root of inverse film thickness. 


\section{Experimental techniques}

In this chapter, the experimental techniques are presented. DC and magnetron sputtering were applied for sample preparation. A setup for bending and emf measurement is described. The onset and propagation of buckling was observed using a light microscope. The geometry of buckles was determined by using a white-light interferometer. Also, peel-off equipment is presented.

\subsection{Sample preparation by sputtering techniques}

\subsubsection{DC sputtering of films}

The samples in this work were prepared using ultra-high-vacuum (UHV) sputter deposition. The principle of the sputtering technique is an ion bombardment of a target, from which atoms are removed and deposited on a substrate. The main part of the sputter system is the argon source, where the argon ions are created and accelerated. The RF generator was operated with the power of $76 \mathrm{~W}$ and a frequency of $13.6 \mathrm{MHz}$ for the argon ionization. Exterior magnetic fields accelerate the argon ions to move on circular orbits. In this process the collisions of gas particles promote ionization and thereby produce plasma. Two grids in the source are used to accelerate and focus the ions in a designated direction towards the target. The first grid raises the potential of the argon plasma to $U_{\mathrm{scr}}=+800 \mathrm{~V}$. The second grid has a potential of $\mathrm{U}_{\mathrm{acc}}=-80 \mathrm{~V}$ for the ions acceleration. The plasma is focusing on the water-cooled target and enables the sputter process from the target on to a substrate. The distance between the target and the substrates was about 10 $\mathrm{cm}$. The basis pressure in the sputter chamber was $<5 \cdot 10^{-10}$ mbar. The argon with a purity of 5.0 and a pressure of about $10^{-4}$ mbar was used during sputtering. The sputter rate for niobium was $0.6 \mathrm{~nm} / \mathrm{min}$ and for palladium $1.2 \mathrm{~nm} / \mathrm{min}$. The sputter process was always started after a $40 \mathrm{~min}$ warm-up of the source and target cleaning.

Thin $\mathrm{Me} / \mathrm{Nb} / \mathrm{Pd}$ film packages were deposited by argon sputtering onto different substrates (bisphenol-A polycarbonate, sapphire). The thickness of the Nb-layer was varied from 50 to 200 $\mathrm{nm}$. The Pd coping layer and the Me(metal) interface layer between substrate and $\mathrm{Nb}$ layer were about 10-20 nm. The Pd underlayer in $\mathrm{Pd} / \mathrm{Nb} / \mathrm{Pd}$ model system was used to prevent oxidation of the $\mathrm{Nb}$-film at the polymer surface, to avoid the change of adhesion due to oxidation and for investigation of $\mathrm{Pd} / / \mathrm{PC}$ bonding. Also the adhesion of $\mathrm{Cr}, \mathrm{Fe}$ and $\mathrm{Mo}$ to $\mathrm{PC}$ was investigated. The thickness of the PC substrates was chosen between $0.02 \mathrm{~mm}$ and $2 \mathrm{~mm}$. The polycarbonate substrates were shortly cleaned with isopropanol prior to sputtering. The metal films were 
prepared at room temperature. Partial pressures of $10^{-8}$ mbar for water and hydrogen were detected during film deposition by using of mass spectrometry [N06]. Other gas partial pressures were even lower. Pd coping layer was deposited on top of the Nb-films to prevent the films from oxidation and to facilitate hydrogen loading.

\subsubsection{Magnetron sputtering}

The preparation of thick niobium films with up to 4.7 micrometer thickness was realized by triode magnetron sputtering in ultra-high-vacuum by a residual gas pressure of $8 \cdot 10^{-8} \mathrm{mbar}$. This technique is the industry widely used sputtering process. In the presence of crossed E- and Bfields generated electrons move on closed orbits above the cathode surface. This increases the collision rate between electrons and the sputter gas. As a result, dense plasma at rather low pressure can be created. The plasma density close to the cathode is high. This results in a high material loss from the cathode. The argon pressure value has a large influence on the deposition process. If the pressure is low, this enables the large free path of sputtered atoms with maintaining their high kinetic energies. The increase of pressure leads to an increase in collisions with the gas. If the free paths of atoms are significantly smaller than the target-substrate distance, a state of thermalization is achieved, i.e. due to the frequency of collisions the kinetic energy of sputtered atoms is close to the thermal energy of the gas molecules, and it is possible to deposit atoms with a relatively small kinetic energy $(<1 \mathrm{eV})$. The basic argon pressure during deposition was about $7 * 10^{-3}$ mbar so that the mean free path of the atoms was only a few centimetres. The distance between the target and the substrate was about $35 \mathrm{~cm}$. The sputter rate for niobium was about $11.5 \mathrm{~nm} / \mathrm{min}$ by a target DC power of $700 \mathrm{~W}$.

\subsection{Emf measurement during hydrogen loading}

The equilibrium voltage in the loading cell, called the electromotive force emf, is the potential difference between the electrodes in an electrochemical cell at currentless equilibrium conditions. The emf is linked to the hydrogen partial pressure at a sample surface via Nernst's equation

$$
U=U_{0}-\frac{R T}{2 F} \ln \frac{p_{H_{2}}}{p_{H_{2}, 0}(T)},
$$


where $U_{0}$ is a reference potential at standard conditions, $F$ is Faradays constant, and $p_{H_{2}, 0}$ is a reference pressure. The chemical potential of the sample is [Sht03]

$$
\mu_{H}=\mu_{H, 0}(T)+R T \ln \frac{p_{H_{2}}}{p_{H_{2}, 0}(T)}
$$

Using (2.1) and (2.2) the relationship between emf and chemical potential follows as $\mu_{H}(U)=-\left(U-U_{0}\right) F$

The reference emf $U_{0}$ practically depends on the properties of the reference electrode and was therefore experimentally determined by bulk measurements.

During the achievement of the thermodynamic equilibrium after hydrogen loading steps the hydrogen concentration in the surface layers of the loaded sample changes due to diffusion. As a consequence, the chemical potential and the electromotive force are changed. The emf was measured as electrical voltage between the sample and an $\mathrm{Ag} / \mathrm{AgCl}$-electrode using an impedance transducer. This allows a currentless measurement to avoid hydrogen loading and unloading of the sample.

\subsection{Bending setup. Two cells for controlled hydrogen loading}

Two cells for controlled hydrogen loading were designed and constructed. The first cell is schematically shown in Fig. 2.1. A sample, contacted for electrochemical hydrogen loading via a glued conductor, has to be clamped on one side.

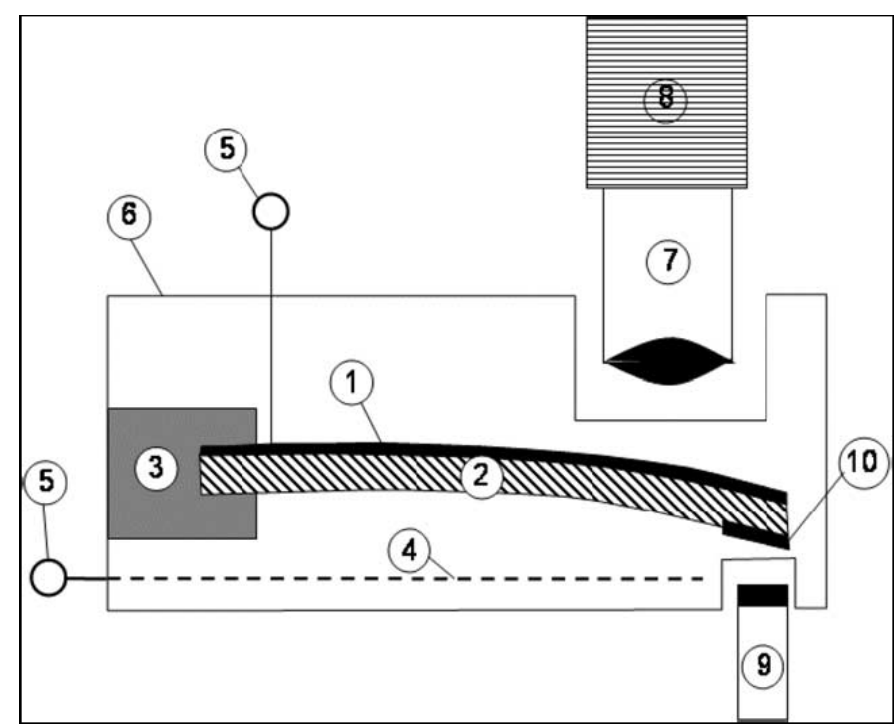

Figure 2.1: Schematic picture of the stress measurement equipment [PNPK04]. The film (1) on substrate (2) is clamped (3) in an electrolytic container (6) which allows electrochemical hydrogen loading of the film via a counter electrode (4) and current leads (5). The deflection of the substrate is monitored with an inductive strain sensor (9) which senses a metal piece (10) attached to the substrate. The film and its buckling are monitored using an optical microscope (7) and a CCD camera (8). 
For the observation of buckle-formation, the electrochemical loading cell including the sample was mounted on an optical microscope stage. The film morphology development during hydrogen loading was monitored in situ with a CCD camera. The advantage of this cell is the possibility to monitor the surface of a sample during hydrogen loading, enabling the desired optical determination of the onset of buckling. This critical point is linked to the critical hydrogen concentration and the critical stress in the hydrogen absorbing layer (see chapter 1.2.4). The monitoring of a sample during hydrogen loading allows the in-situ observation of the buckles' morphology, buckled areas, which indicate places with a poor adhesion, etc. The disadvantage of such construction is the effect of buoyancy of the electrolyte onto the sample. The density of the electrolyte is about $1.4 \mathrm{~g} / \mathrm{cm}^{3}$. Therefore the buoyancy force on a sample with $30 \times 7 \times 1 \mathrm{~mm}^{3}$ dimension is about $2.9 \mathrm{mN}$ calculated by using of Archimedes principle. But this force is comparatively small to the forces acting on the metal layer during hydrogen loading.

A highly viscous electrolyte was prepared by mixing two volume parts of glycerine with one volume part of phosphoric acid (85\%). This electrolyte is hygroscopic and has to be renewed after several measurements. The high viscosity of the electrolyte decreases the mobility of molecular oxygen in the electrolyte and thereby reduces hydrogen desorption from the film surface. Additionally, the electrolyte was bubbled with argon before using to keep the amount of oxygen in the electrolyte small. Before loading, the $\mathrm{Nb}$-samples were unloaded with a constant voltage of $0.4 \mathrm{~V}$ for the time interval $t=10 \mathrm{~h}$. The discharge voltage should not be too large, since otherwise oxidation of the sample might occur and the electrolyte might dissociate [Lau98].

The stresses evolving in the metal layer during hydrogen loading were measured by determination of the deflection of the substrate due to film expansion. Therefore, the substrate was clamped at one side. The bending of the substrate was measured by using of a strain gauge mounted at the other side of the substrate. During hydrogen loading, the expanding film bends the substrate, as it is shown in Fig. 2.1. The vertical movement of the end of the sample during hydrogen loading is measured by an inductive sensor. The sensor consists of a coil which is at the same time a part of a resonant circuit. If such sensor is fixed close to a moving surface of a metal, e.g. a plate, then the induction changes as a function of the distance to the object. In the metal plate eddy currents are formed as a result from magnetic induction. According to the Lenz rule the eddy currents in the metal plate are directed in the way, so that they try to weaken the alternating current in the coil. The size of the eddy currents induced in the metal plate depends on the distance between coil and metal plate providing a contactless inductive displacement measurement. The sensor can be calibrated by using different metal plates. Substrate thickness and length have been optimized in order to be able to measure with the inductive sensor. In our case a sensor with a measuring range of $1 \mathrm{~mm}$ was used. So, for the bending by hydrogen loading, 
niobium films with $50-200 \mathrm{~nm}$ thickness on polycarbonate substrate with $30 \mathrm{~mm}$ length, $7 \mathrm{~mm}$ width and $0.25-2 \mathrm{~mm}$ thickness were chosen. Such geometry of the samples has the proper length-to-width ratio for using Stoney's formula (see below). It has an appropriate area for sputter deposition and its bending during hydrogen loading is not larger than the measuring range of the inductive sensor.

The movement of the sample during "bending up" and "bending down" is shown in Fig. 2.2.
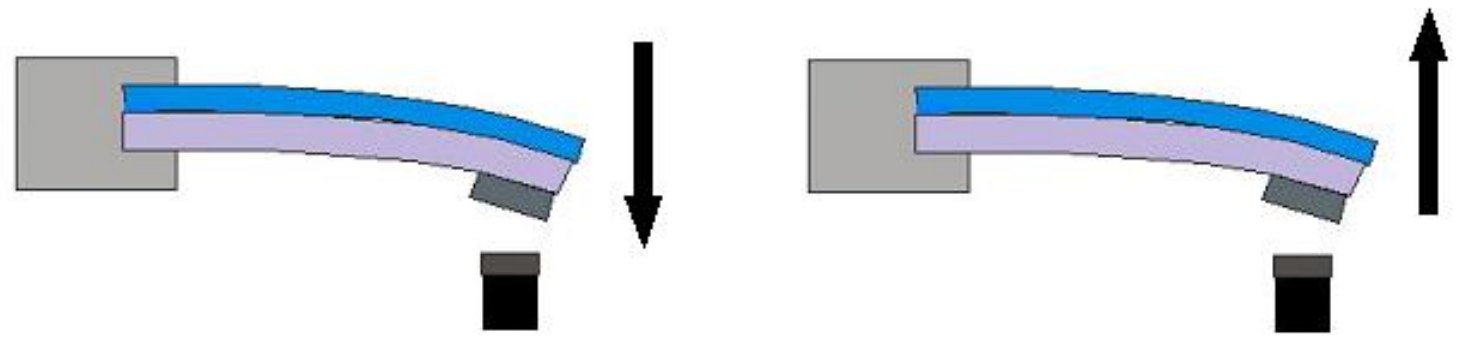

Figure 2.2: Schematical picture of sample bending. a) Bending down, this results in the negative slope in a stress-hydrogen concentration curve. b) Bending up, the slope is positive.

The sample can move upwards and downwards, while for each moving direction definite physical processes are responsible, that have been identified in this work. The calibration curve for the sensor I-W-A-/A4 (Amos company) against a Pd- plate is shown in Fig. 2.3.

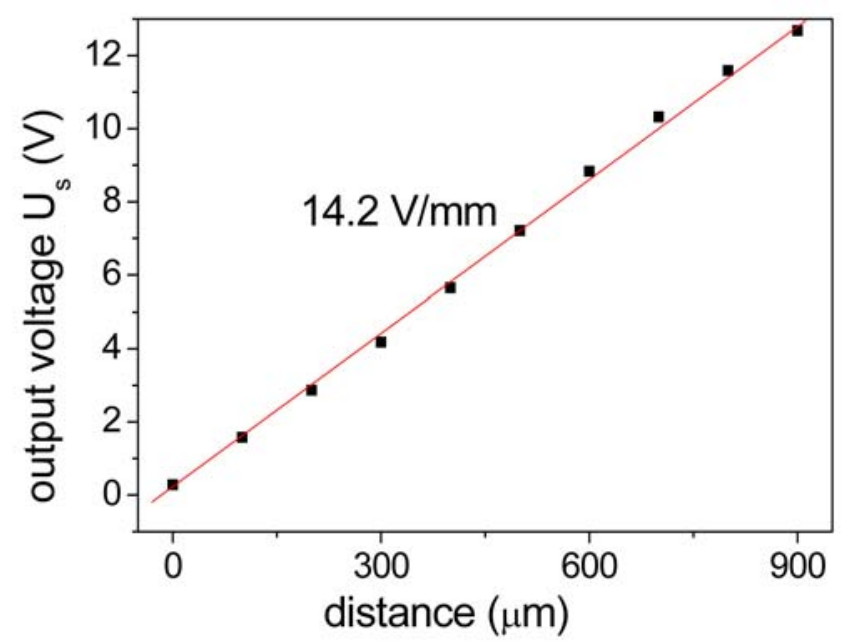

Figure 2.3: Calibration curve of the sensor I-W-A-/A4 against a Pd-plate.

The X-scale in this plot is a distance between the sensor and a Pd-plate. The linear change of the output voltage in the whole measuring range of the sensor can be seen.

The second cell, which was used for electrochemical hydrogen loading and stress measurement, is shown in Fig. 2.4. 


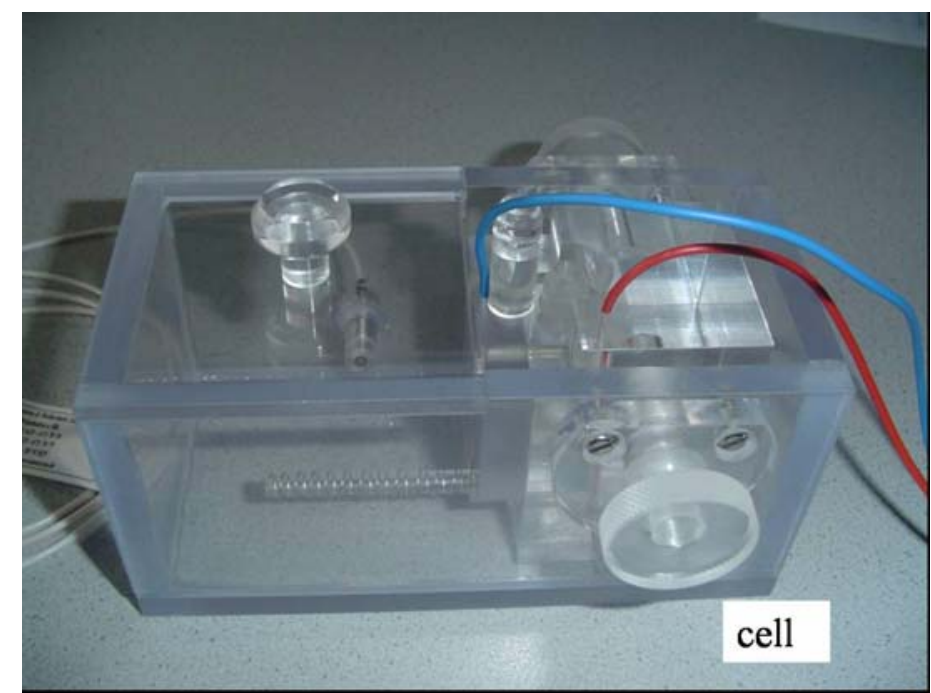

Figure 2.4: The second cell for controlled hydrogen loading, prepared for exhibition during Hanover Trade Fair 2006. The blue wire is for the contact with a sample, the red wire is contacted with the counter electrode.

The second cell was constructed for presentation during an exhibition in Hannover and it was made from acrylic glass. This cell allows samples to be mounted and removed quickly. With a special clamping construction it is possible to contact a sample to electrical supply by pressing a needle onto it, which is connected with a wire and sealed with a rubber for protection from electrolyte. The sample can be moved to the inductive sensor in order to be in the measurement range of the inductive sensor. The disadvantage for this cell is that it is impossible to watch the surface of the sample using the light microscope during the measurement. Because of that the first cell was mainly used.

2.4. Buckles observation and determination of their shape for adhesion determination. Shape of buckles as a measure of adhesion

In the following chapter an introduction into the method of adhesion determination by investigation of the buckle geometry is presented. Also equipment for the peel measurements is shown. 


\subsubsection{Gille and Rau theory}

The theory of Gille und Rau determines the adhesion energy from the height $h$ and width $l$ of a buckle (Fig. 2.5). Gille and Rau [GR84] have shown that the cross-sectional buckle morphology contains information about film properties, such as the film's elastic modulus and the adhesion energy between the film and the substrate. The adhesion energy $\gamma$ of a film can be calculated by measuring the geometry of a detached area from the following relation (cf. Fig. 2.5)

$$
\gamma=\left(\frac{6}{5}\right)^{2} \frac{\mathrm{Ed}}{1-v^{2}}\left(\frac{\mathrm{h}}{1}\right)^{4}\left[1+\left(\frac{5}{3}\right)^{2}\left(\frac{\mathrm{d}}{\mathrm{h}}\right)^{2}\left\{1-\frac{25}{12}\left(\frac{\mathrm{d}}{\mathrm{h}}\right)^{2}\right\}\right] .
$$

Gille and Rau assumed a straight sided buckle of uniform width and height with the crosssectional geometry

$$
z=f(x)=12 h\left(\frac{x}{1}\right)^{2}\left(1-\frac{4 x}{31}\right)
$$

with height $z$, extension $x$ and $0 \leq x \leq l$, as well as purely elastic film buckling. Coupeau [CNC99] and Matuda [MBK81] determined a sinusoidal cross-sectional geometry of a section of one buckle. However, according to Gille and Rau [GR84], Eq. (2.3) changes only slightly if the assumed shape of a buckle takes another form and the resulting adhesion energies are similar.

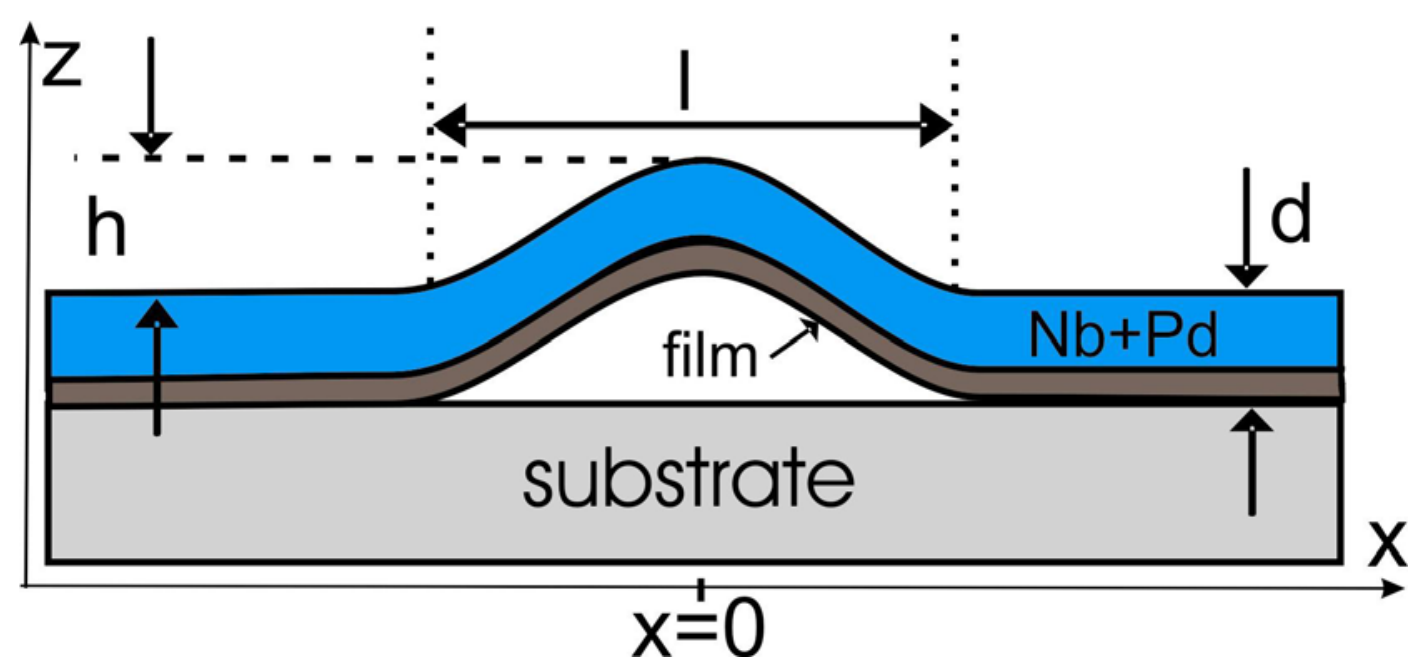

Figure 2.5: Cross-sectional view of a straight-sided buckle as described by Gille and Rau [GR84] resulting from mechanical stress between the substrate and the film of thickness $d$. Buckle height $h$ and buckle width $I$ is controlled by the adhesion energy. 


\subsubsection{White-light interferometry}

In cooperation with Mahr Company the shape of the buckles was measured with a newly designed white-light interferometer (Mahr) [DG94]. The optical setup of the interferometer is similar to the classical Mirau-interferometer. A white light source with low coherency is used. The reference field of the interferometer is integrated in a Mirau objective. During the measurement, the Mirau objective is moved in a direction vertical to the interesting surface, resulting in interferograms of several focussing depths. This sequence of images is used to analyse the measured signals by special software to calculate the 3-D topography of the object. Theoretically, a vertical height resolution of $0.1 \mathrm{~nm}$ can be achieved by this method. Limited by the lateral resolution of the used optical lenses and the CCD-camera, this height resolution can be reduced by lateral sample roughness. In this work, the experimental vertical resolution was \pm 10 $\mathrm{nm}$ [DKG03]. The material of the investigated object is irrelevant for the measurements, but it should be not transparent. By use of an objective $(20 \times)$ this technique allows to study a large sample section of $0.8 \times 0.6 \mathrm{~mm}^{2}$, with a lateral sampling interval of $1.25 \mu \mathrm{m}$. Furthermore, this technique works non-contacting and thus denting of the sample can be excluded. The white-light interferometer is shown in Fig. 2.6.
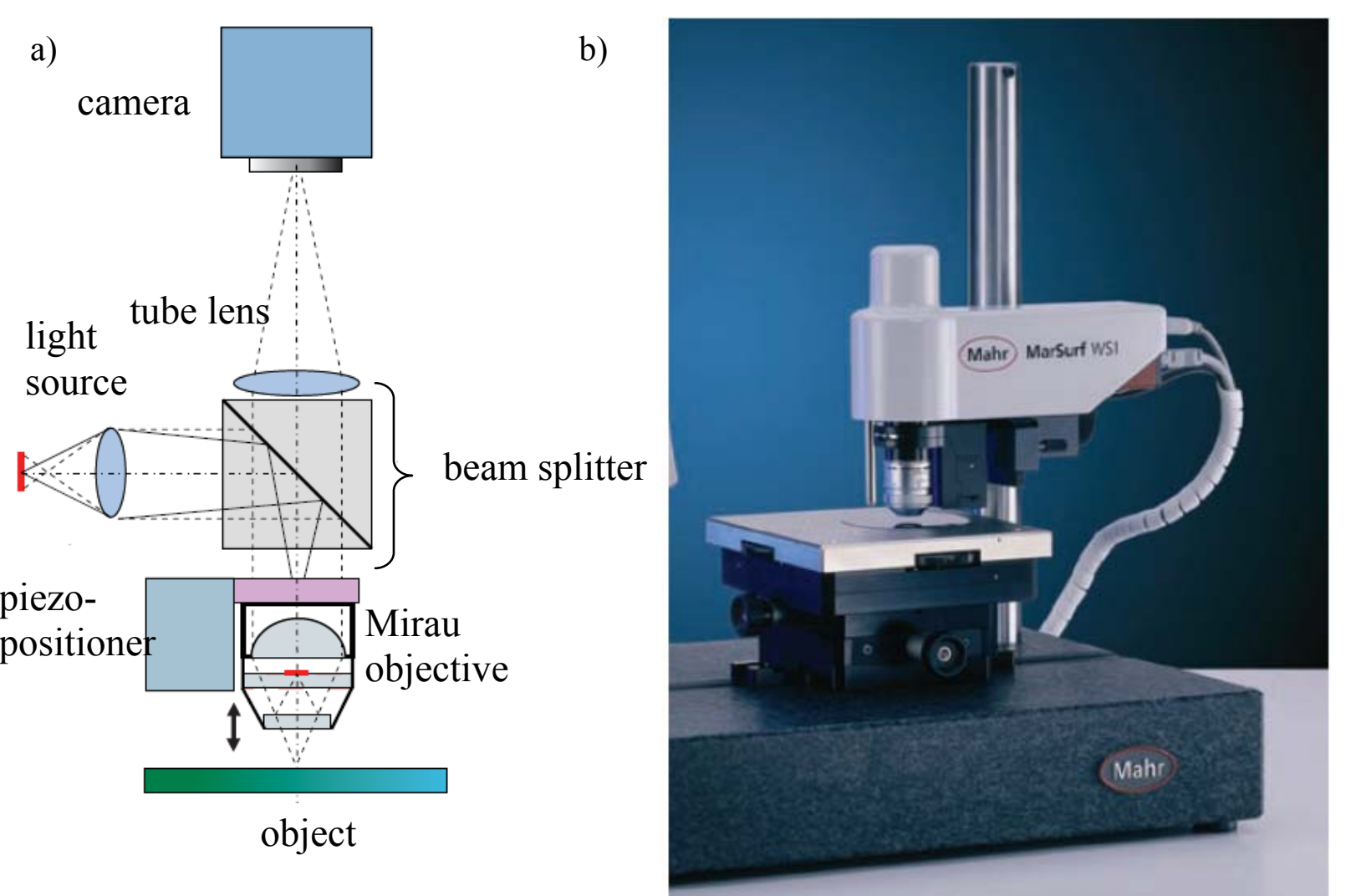

Figure 2.6: a) Principle of a Mirau type white-light interferometer, b) Photo of the white-light interferometer MarSurf WS 1 produced by Mahr GmbH for the nonimpact measurement of the surface structures (taken from MarSurf WS 1 - Flyer, www.mahr.de). 
Buckle shapes can also be obtained by surface sensing techniques like atomic force microscopy or scanning tunnelling microscopy. Because of the limited section monitored by these methods compared to the rather large size of buckles, these AFM- and STM-techniques usually just show a small fraction of the buckles. This makes it difficult to differentiate a pure straight sided buckle from a fraction of a buckle that looks like as if it were a straight sided buckle. Therefore, this technique has not been applied in this work.

\subsubsection{Light microscopy}

The buckling process during hydrogen loading was studied using a Leitz Metallux II optical microscope (light microscope). This is a compound microscope that uses multiple lenses to further increase magnification. The magnification values of objective lenses are $5 \mathrm{x}, 10 \mathrm{x}, 20 \mathrm{x}$, 50x and 100x. The surface-observation during hydrogen loading was performed using 10x objective lenses. This allows to see a surface segment of about $750 \times 900 \mu^{2}$. The objective lens was protected with Plexiglas from electrolyte. This was possible because of the large focal distance of this objective lens. The microscope has been supplied with a digital camera that was used to record digital images of the sample surfaces before, during and after hydrogen loading.

In the optical microscope, buckles show off as dark lines. These dark lines can be related to local film detachment and crack formation, since the film in this area is lifted above the original level. This was verified by changing the light conditions, by interference microscopy and by white light interferometry. An example of how the buckles look like is shown in light microscopy images in Fig. 3.1. This figure presents the images of a buckled $300 \mathrm{~nm} \mathrm{Nb}$ film, observed with different magnifications. It can be seen that the low magnifications and therefore large focal distances of oculars lead to a manifestation of buckles as dark lines as a consequence of light-reflection at the sides of buckles and decreasing light intensity coming from the buckles into the ocular. Despite of buckling, the film is still intact and does not contain holes or cracks. This can be seen in Fig. 2.7 d), the image with the largest magnification. 
a)

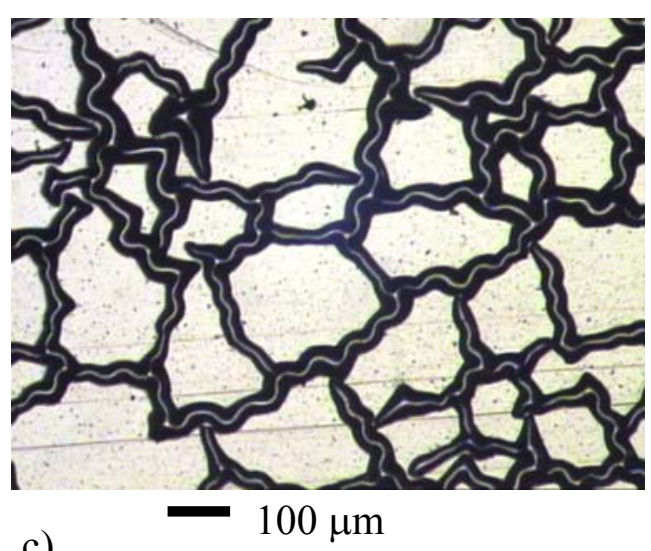

c)

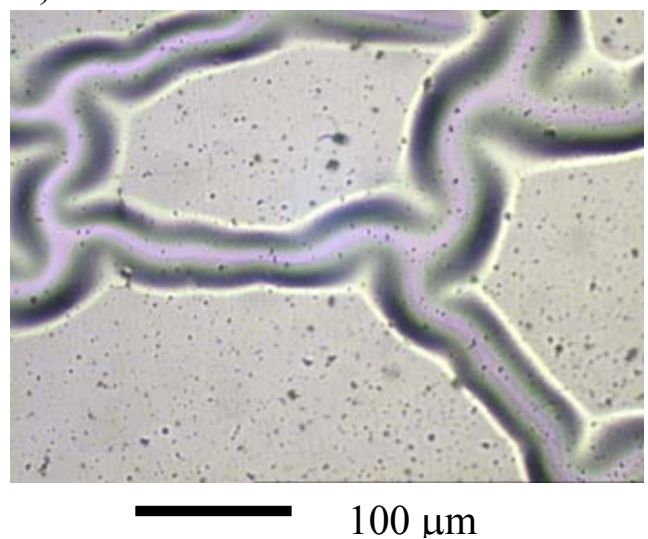

b)

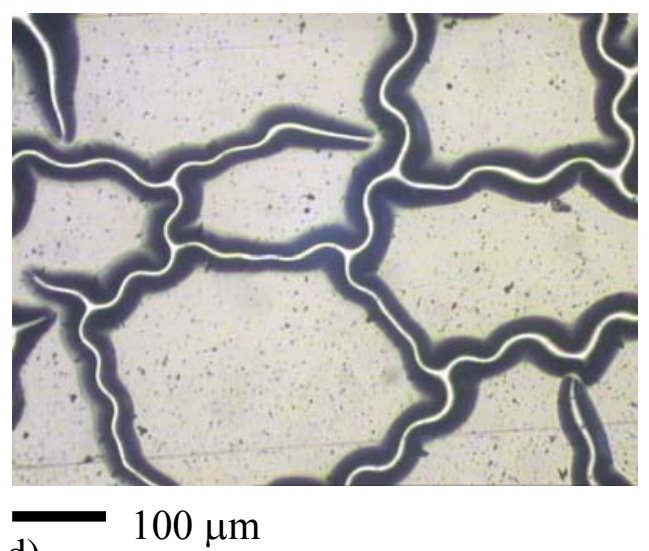

d)

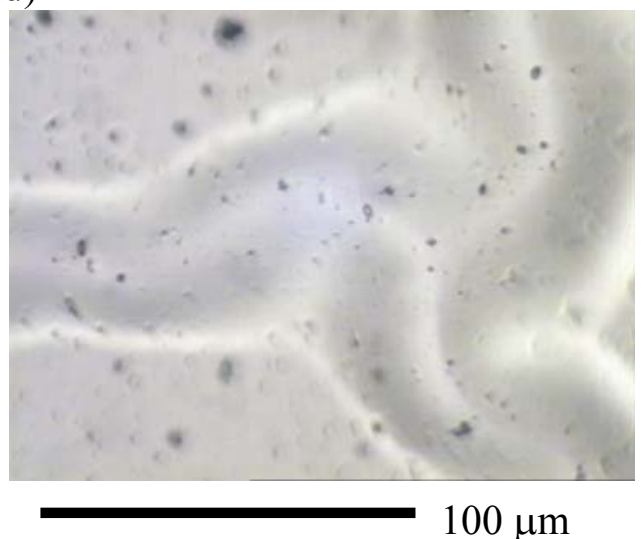

Figure 2.7: Buckles of a $300 \mathrm{~nm}$ Pd film. Light microscopy pictures recorded from the same place of the surface with different magnifications. The pictures verify that the film is still intact but delaminated. Dark lines represent film buckles.

The buckle geometry can be obtained by using interference microscopy, as well, as it is shown in Fig. 2.8. Adjacent two dark (white) lines correspond to a height difference of $0.27 \mu \mathrm{m}$ by using of monochromatic thallium light.

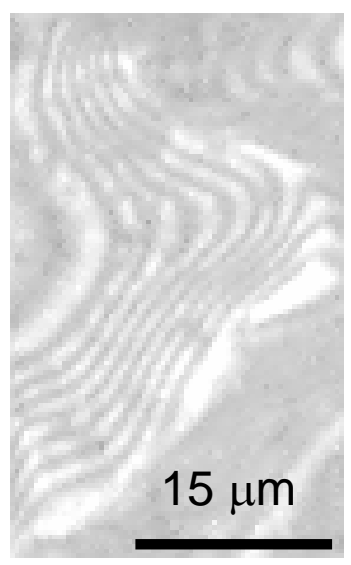

Figure 2.8: interference image of a buckle measured of a Pd/Nb(200 nm)/Pd/PC(1 mm) sample after loading up to $100 \% \mathrm{H} / \mathrm{Nb}$. Adjacent two dark (white) lines correspond to a height difference of 0.27 $\mu \mathrm{m}$. 


\subsection{Peel test}

In cooperation with the group of Prof. F. Faupel, University of Kiel, peel-of measurements at model samples were carried out. With this procedure the samples were peeled from the substrate and the peel-off strength was measured.

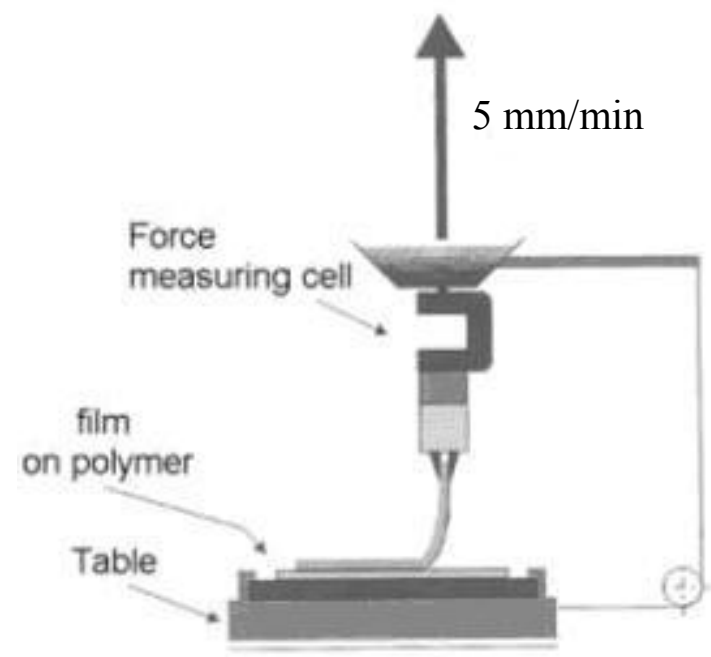

Figure 2.9: Peel-off equipment (schematic).

The fracture energy $\gamma_{\mathrm{a}}$ in this experiment is a function of a peel angle, it is [K71]

$$
\gamma_{a}=\frac{F(1-\cos \alpha)}{w}
$$

where $\alpha$ is the peel angle, $w$ is the width of a peeled film and $F$ the peeling force . During peeling the substrate is moved in a way that film pulling always takes place perpendicularly to the substrate surface. This is possible due to a special construction of the table where the sample is fixed on. The samples were stabilized either with a thick $\mathrm{Cu}$-layer of approx. $4 \mu \mathrm{m}$ and then with an adhesive tape or they were simply covered with an adhesive tape. The peeling with a tape can only be successful if the bonding of the tape to a metal film is stronger than that between the film and a substrate. The width of the films was $6 \mathrm{~mm}$. To be able to remove the film from the substrate a boundary region was contaminated with finger fat on one side by approx. $1 \mathrm{~mm}$ of the substrate (30 mm long) before sputtering the film. It is not possible to calculate the adhesion energy directly from the peel strength, because of the large energy dissipation during the plastic deformation of the peeled films. Therefore, only qualitative conclusions will be made. 


\section{Experimental results}

Electrochemical hydrogen loading of metal films on polymer substrates and sapphire was used to investigate the buckling behaviour of the films and to calculate their adhesion energy. Also the following topics were considered: the investigation of cracking interface, the determination of critical values for buckling and different secondary effects by electrochemical hydrogen loading with the goal of stress measurement optimisation.

\subsection{Hydrogen loading of $\mathrm{Pd} / \mathrm{Nb} / \mathrm{Pd}$ films on polycarbonate}

In this chapter the results of buckle formation by using of optical observations and stress measurements during hydrogen loading are presented. The influence of the film thickness and compliance of substrate was also investigated.

\subsubsection{Buckle formation at the critical hydrogen concentration}

$\mathrm{Pd} / \mathrm{Nb} / \mathrm{Pd}$ on polycarbonate (PC) was used as a model system for the investigation of buckling during hydrogen loading. The thickness of the Nb-layer was varied from 50 to $200 \mathrm{~nm}$. The Pd coping layer comprised about 10-20 $\mathrm{nm}$ on both sides of the $\mathrm{Nb}$ film. The hydrogen concentration was calculated assuming the location of hydrogen atoms only in the Nb-layer, see chapter 2.

In the model of adhesion energy determination applying the controlled hydrogen loading technique (Chap. 1.2) it is assumed that the buckle formation starts locally, i.e. as soon as the elastic energy stored in $\mathrm{Nb}-\mathrm{H}$ by H-loading becomes larger than the adhesion energy. The onset of delamination via buckle formation would be expected by exceeding the critical Hconcentration. In order to confirm this assumption stepwise loading experiments at different samples were carried out. It was found that the buckle density rises sharply within a concentration step of $0.01 \mathrm{H} / \mathrm{Nb}$. The change of the surface morphology during stepwise hydrogen loading is shown in Fig. 3.1, where the controlled H-loading of a $200 \mathrm{~nm} \mathrm{Nb}$-layer on $0.5 \mathrm{~mm}$ PC with loading steps of $\Delta \mathrm{c}_{\mathrm{H}}=0.01 \mathrm{H} / \mathrm{Nb}$ is presented. With low magnification, the asdeposited 200-nm Nb-film surface looks smooth, as shown in Fig. 3.1(a). By a stepwise increase of the hydrogen concentration by $0.01 \mathrm{H} / \mathrm{Nb}$, the in-plane stress increases. Above a hydrogen concentration of $0.08 \mathrm{H} / \mathrm{Nb}$, buckling develops very quickly. 
a) $0 \% \mathrm{H} / \mathrm{Nb}$

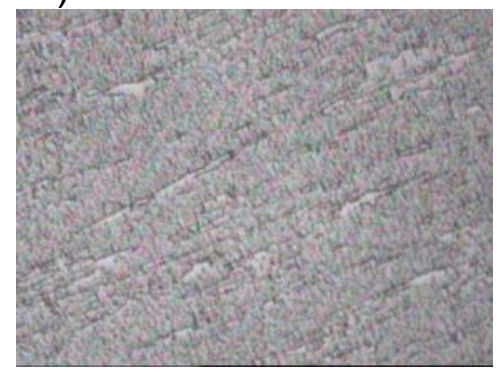

d) $10 \% \mathrm{H} / \mathrm{Nb}$

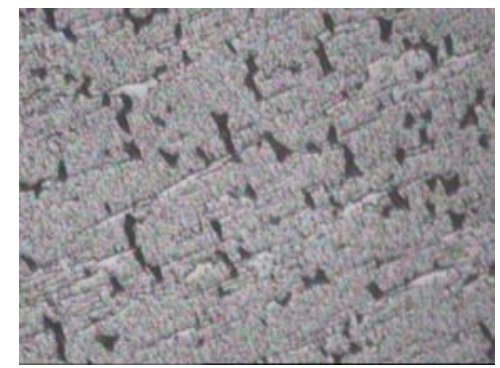

b) $7 \% \mathrm{H} / \mathrm{Nb}$

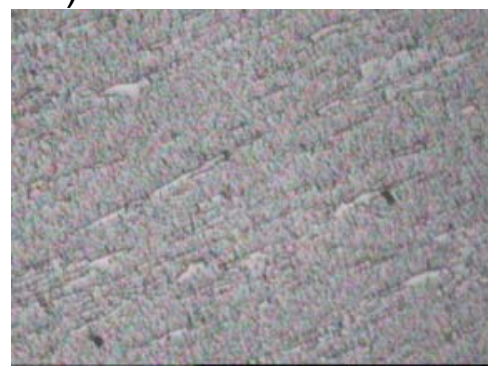

e) $15 \% \mathrm{H} / \mathrm{Nb}$

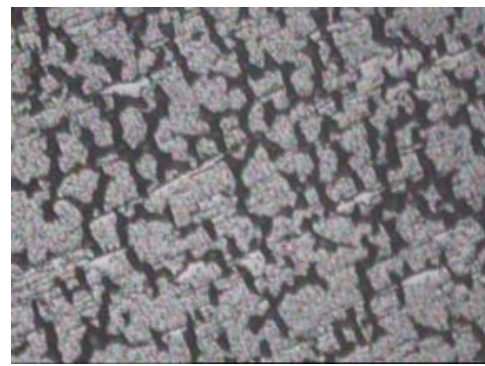

c) $8 \% \mathrm{H} / \mathrm{Nb}$

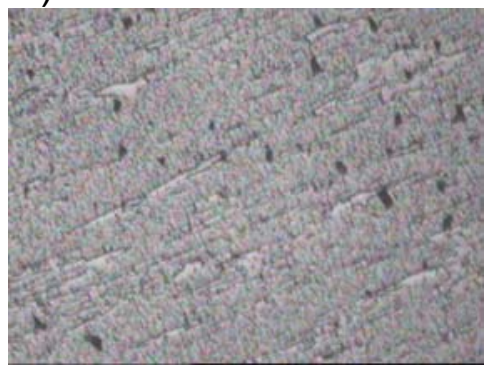

f) $20 \% \mathrm{H} / \mathrm{Nb}$

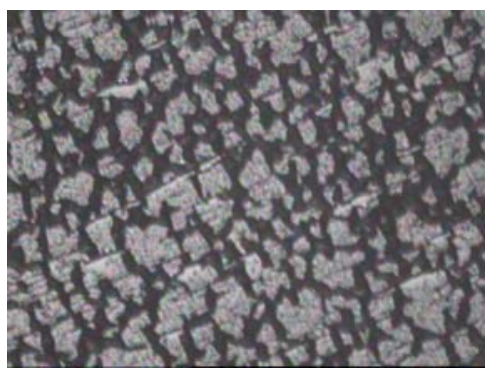

$100 \mu \mathrm{m}$

Figure 3.1: Surface of the sample $\operatorname{Pd}(10 \mathrm{~nm}) / \mathrm{Nb}(200 \mathrm{~nm}) / \operatorname{Pd}(10 \mathrm{~nm}) / / \mathrm{PC}(0.5 \mathrm{~mm})$ at different hydrogen concentrations ( $\mathrm{a}-\mathrm{f}$ ) given as the atomic ratio $\mathrm{H} / \mathrm{Nb}$. The bar corresponds to a length of 100 $\mu \mathrm{m}$.

These light microscopy top view images show a strong increase of the delaminated film fraction during one loading step from 0.07 to $0.08 \mathrm{H} / \mathrm{Nb}$. Therefore, the hydrogen concentration of $8 \%$ $\mathrm{H} / \mathrm{Nb}$ can be defined as the critical hydrogen concentration for delamination. The quantitative evaluation of the critical hydrogen concentration can be obtained by counting the pixels in the dark regions of the light microscopy images. This is possible by applying image analysis software. In Fig. 3.2 the delaminated film surface fraction for increasing hydrogen concentration is shown, as it was determined from the images in Fig. 3.1. 


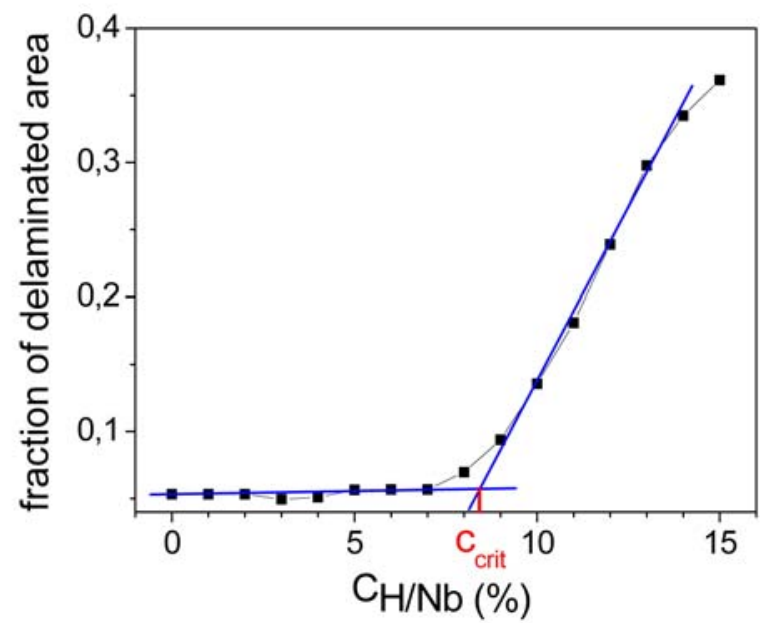

Figure 3.2: Delaminated surface fractions of a $\operatorname{Pd}(10 \mathrm{~nm}) / \mathrm{Nb}(200 \mathrm{~nm}) / \mathrm{Pd}(10 \mathrm{~nm}) / / \mathrm{PC}(0.5 \mathrm{~mm})$ film obtained by pixel counting in the black areas in the light microscopy images of Fig. 3.1. It can bee seen that delamination abruptly sets in at about $0.084 \mathrm{H} / \mathrm{Nb}$.

The increase of the debonded film area is approximated by a straight line. Its intercept with the line representing the initial fraction of the delaminated area yields the critical concentration for delamination of the film. In this case it is $\mathrm{c}_{\mathrm{crit}}=8.4 \% \mathrm{H} / \mathrm{Nb}$.

Another possibility to obtain the critical hydrogen concentration for buckling is the measurement of the mechanical stress evolution in the film during hydrogen loading. The stress curve of $\operatorname{Pd}(10 \mathrm{~nm}) / \mathrm{Nb}(200 \mathrm{~nm}) / \mathrm{Pd}(10 \mathrm{~nm})$ film on $0.5 \mathrm{~mm} \mathrm{PC}$ can be seen in Fig. 3.3. Results in Figs. 3.2 and 3.3 are from the same sample.

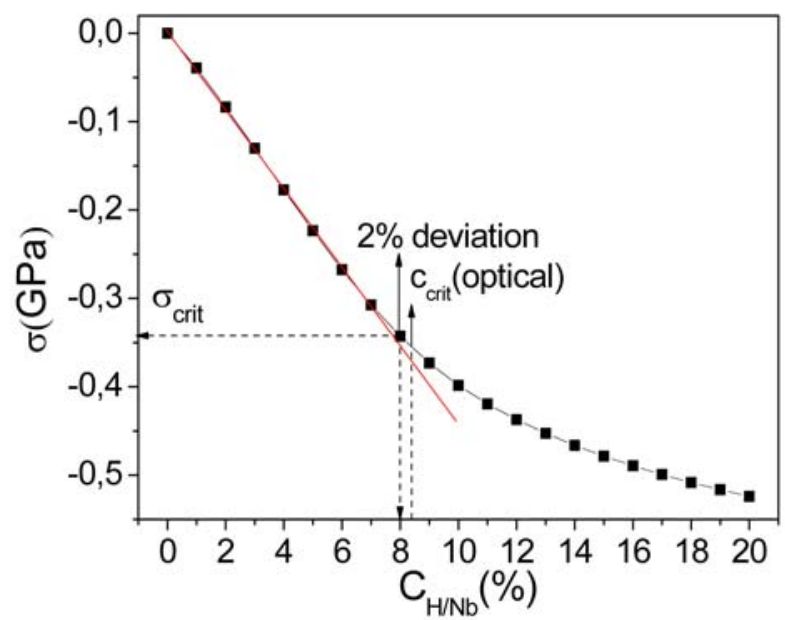

Figure 3.3: Stress curve of $\operatorname{Pd}(10 \mathrm{~nm}) / \mathrm{Nb}(200 \mathrm{~nm}) / \operatorname{Pd}(10 \mathrm{~nm})$ film as a function of hydrogen concentration by stepwise (1\% H/Nb steps) hydrogen loading. $c_{\text {crit }}$ for buckling determined by deviation of $2 \%$ from the linear dependency of the stress curve and optically (see Fig. 3.2) is shown.

The first point of the stress curve that deviates from the initial linear stress evolution shows $2 \%$ positive stress-deviation for $\mathrm{c}_{\mathrm{H}}=8 \% \mathrm{H} / \mathrm{Nb}$. This is due to stress relaxation during buckling, and the determined critical hydrogen concentration is in good agreement with the optically obtained 
value. $2 \%$ deviation was used in this work for the determination of the critical stress and critical hydrogen concentration for buckling.

\subsubsection{Influence of film thickness on critical concentration and critical stress}

The influence of the film thickness on the critical system parameters was investigated for $\mathrm{Nb}$ films with thicknesses between $50 \mathrm{~nm}$ and $200 \mathrm{~nm}$ on PC substrates with thicknesses of $0.5 \mathrm{~mm}$ and $1 \mathrm{~mm}$. According to theory a linear dependency of $\mathrm{c}_{\text {crit }}$ and $\sigma_{\text {crit }}$ for buckling to the square root of inverse film thickness is expected. Thus the critical hydrogen concentration and critical stress for buckling should be smaller for films with larger thickness (see Eq. (1.34)).

In Fig. 3.4, a), b) results on the stress evolution in $\mathrm{Pd} / \mathrm{Nb} / \mathrm{Pd}$ with $50 \mathrm{~nm}$ and $100 \mathrm{~nm} \mathrm{Nb}$ thicknesses, deposited onto $0.5 \mathrm{~mm}$ PC, are shown.

a)

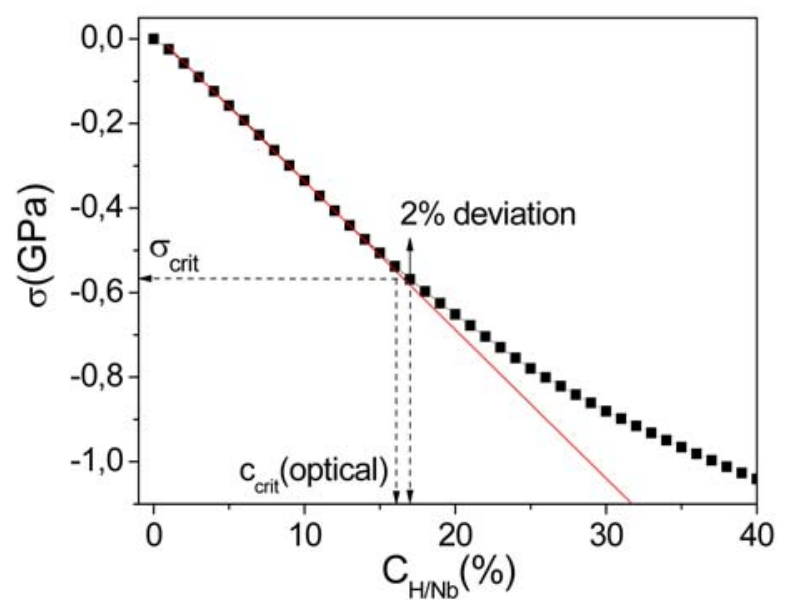

b)

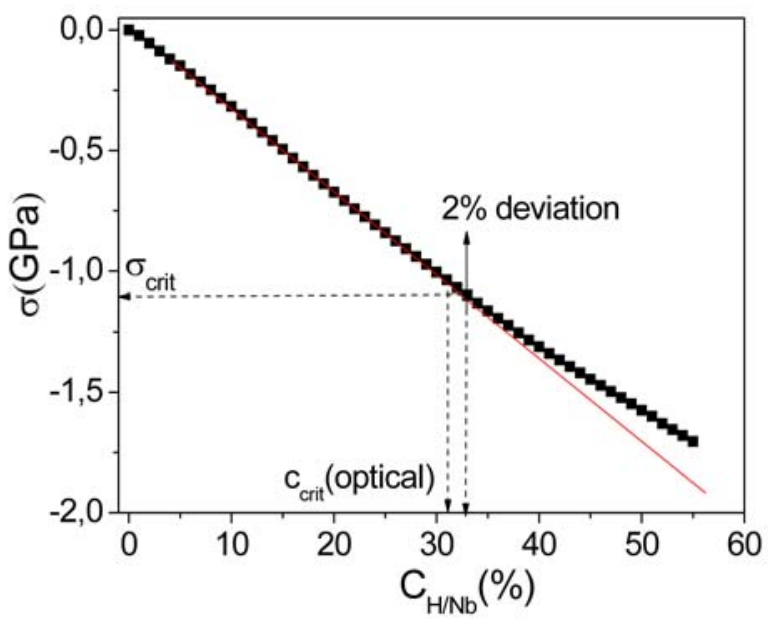

c)

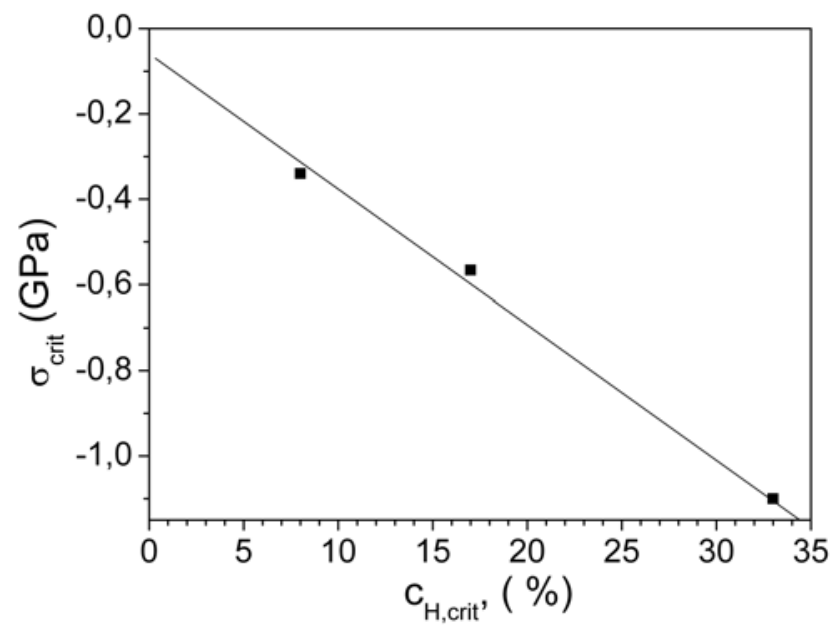

Figure 3.4: Compressive stress within the 100-nm thick Nb-film as a function of hydrogen concentration. The metal film was deposited on a 0.5-mm thick PC-substrate. The onset of buckling of the film is observed when the measured stress deviates from the linear relationship extrapolated from the initial behaviour at low $\mathrm{H}$ concentrations; (a) $100 \mathrm{~nm} \mathrm{Nb}$ film (b) $50 \mathrm{~nm} \mathrm{Nb}$ film, (c) critical stress versus hydrogen concentration 
In all cases compressive stresses increase linearly with increasing hydrogen concentration at the beginning of the loading sequence, as expected from linear elastic theory. The slope of the corresponding straight parts in Fig. 3.4 are in agreement with previous measurements of $\mathrm{Nb}$ on Si-substrates and their theoretical interpretation based on Eq. (1.6) [B98]. The critical stress was set to the value where the stress curve deviates from the linear increase by $2 \%$. In chapter 3.1 .1 it has been shown that the critical hydrogen concentration of $200 \mathrm{~nm} \mathrm{Nb}$ is $8 \% \mathrm{H} / \mathrm{Nb}$ (Fig. 3.1c). For the $100 \mathrm{~nm} \mathrm{Nb}$-layer a crit of $17 \% \mathrm{H} / \mathrm{Nb}$ (optically $16 \%$ ) is observed, for $50 \mathrm{~nm}$ Nb-layer this value increases to $33 \% \mathrm{H} / \mathrm{Nb}$ (optically $31 \%$ ). The initial stress increase is $3.4-4.5 \mathrm{GPa} / \mathrm{c}_{\mathrm{H}}$. Thinner films debond from their substrates at higher hydrogen concentrations and larger stress.

To investigate the influence of the substrate thickness on critical stress and critical hydrogen concentration $\mathrm{Pd}(10 \mathrm{~nm}) / \mathrm{Nb} / \mathrm{Pd}(10 \mathrm{~nm}) / / \mathrm{PC}(1 \mathrm{~mm})$ films with Nb-layer of 50-, 100- and 200$\mathrm{nm}$ thickness have been measured. The stress curves of these samples are shown in Fig. 3.5 for stepwise hydrogen loading.

a)

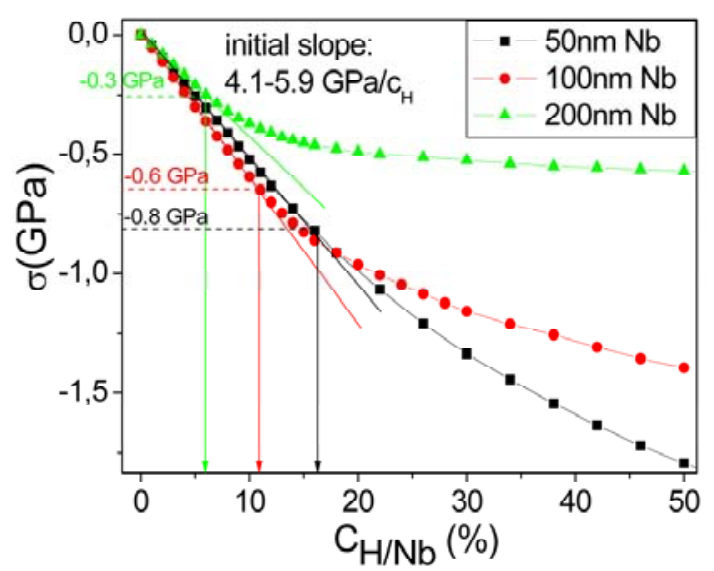

b)

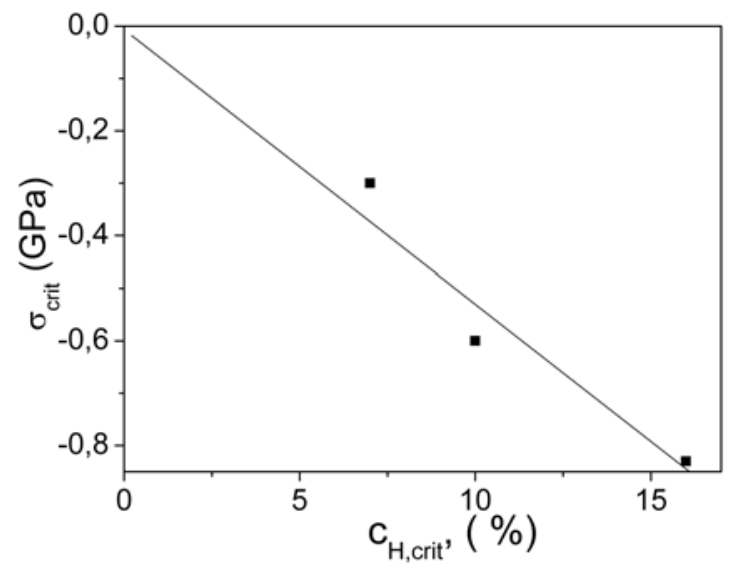

Figure 3.5: Increase of the mechanical stresses in a Pd/Nb/Pd//PC(1 mm) multilayer with different $\mathrm{Nb}$ layer thicknesses (50-200 Nm). The critical stress clearly decreases with decreasing $\mathrm{Nb}$ thickness. The arrows mark the concentrations at which formation of wrinkles was detected by optical lightmicroscopy.

The critical concentration for $200 \mathrm{~nm}$ film was $7 \% \mathrm{H} / \mathrm{Nb}$, for $100 \mathrm{~nm}$ film $11 \%$, for $50 \mathrm{~nm}$ film $17 \%$, determined by $2 \%$ stress-deviation from the initial linear stress increase.

Similar initial slope of the stress for all films on the substrates with the same thickness was expected, but it deviates from sample to sample and amounts 4.1-5.9 GPa/ $\mathrm{c}_{\mathrm{H}}$. These deviations might occur due to different microstructure of the substrates or /and due to the small differences in the substrate thickness, for example. Laudahn [Lau98] has measured for $\mathrm{Nb}$ on silicon the initial linear stress change a value of $6-7 \mathrm{GPa} / \mathrm{c}_{\mathrm{H}}$. 
In Fig. 3.6 the evolution of the buckles morphologies during hydrogen loading is shown for the $\mathrm{Nb}$ films for which the stress measurement has been presented in Fig. 3.5. The width of the buckles is smaller for the thinner films, while the shape of the buckles is similar. The buckles morphology corresponds to the straight-sided Euler buckle morphology.

The light microscopical images confirm the critical hydrogen concentration for delamination derived from the stress curves.

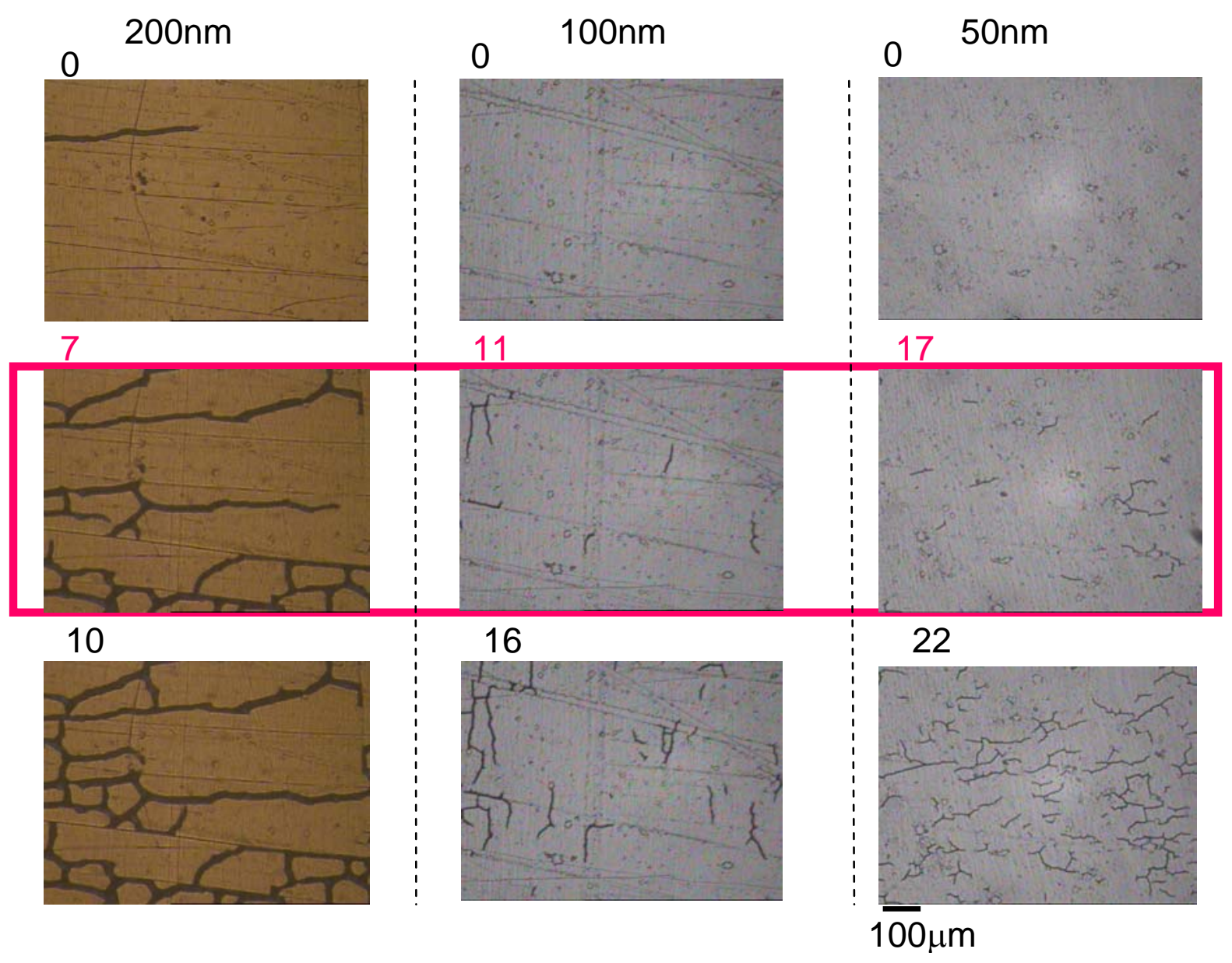

Figure 3.6: Comparison of the buckle morphology during hydrogen loading for Pd/Nb/Pd films with Pd 20nm/ Nb $50 \mathrm{~nm}$ (left), $100 \mathrm{~nm}$ (middle), $200 \mathrm{~nm}$ (right)/ Pd 20nm on 1mm PC. The buckle morphologies at the critical hydrogen concentrations are marked with the red rectangle. The hydrogen content in the metal film is increasing from top to bottom, as marked above each image.

The evaluation of the critical H-concentration for the $\mathrm{Pd}(10 \mathrm{~nm}) / \mathrm{Nb} / \mathrm{Pd}(10 \mathrm{~nm}) / / \mathrm{PC}(1 \mathrm{~mm})$ films from light microscopy is shown in Fig. 3.7. 
a)

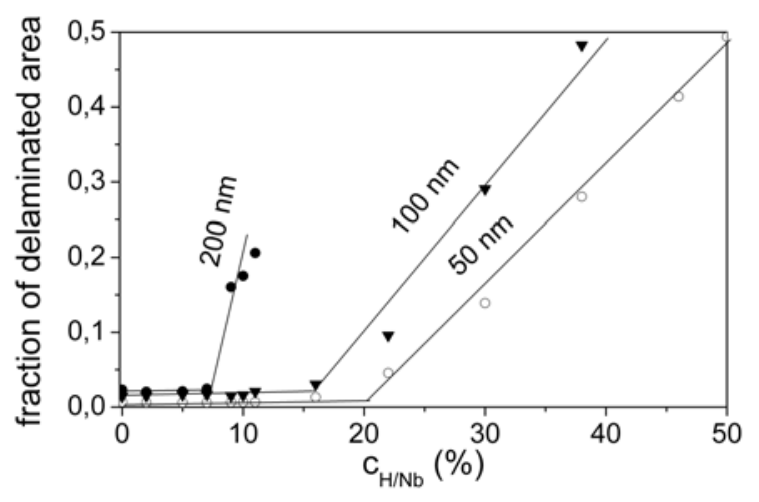

b)

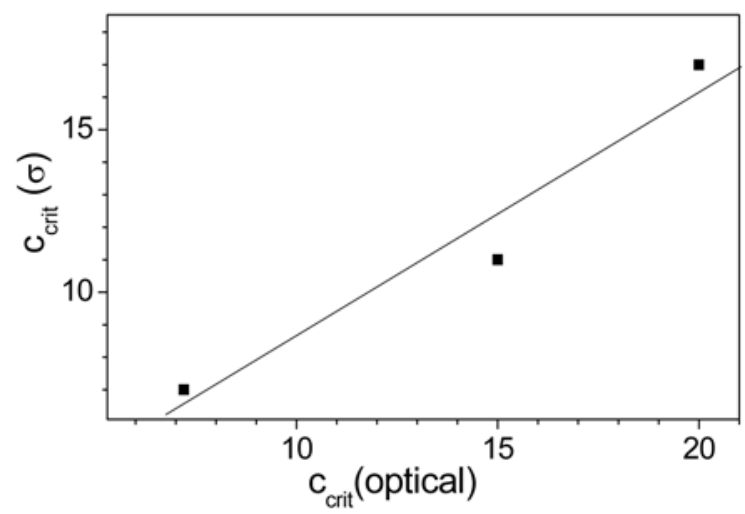

c)

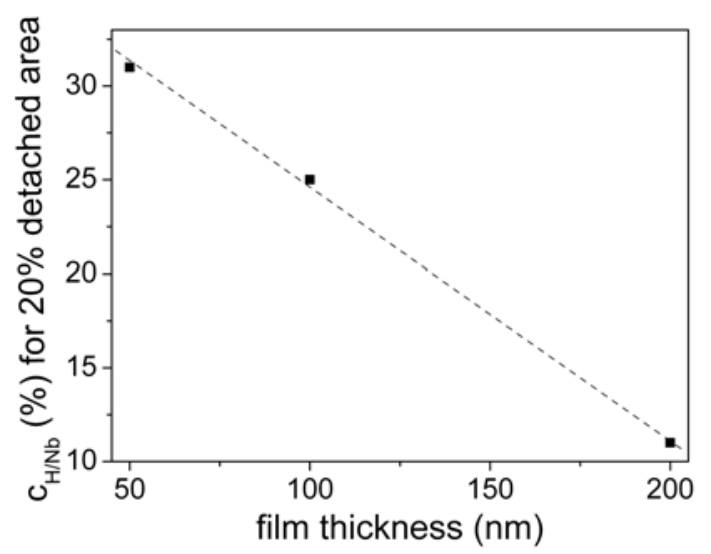

Figure 3.7: a) Fraction of delaminated area of metal films on 1-mm thick PC substrate as a function of H-concentration. The thicknesses of the $N b$ layers are $d=200 \mathrm{~nm}$ (closed circles), $d=100 \mathrm{~nm}$ (triangles) and $d=50 \mathrm{~nm}$ (open circles). The increasing area of detached film is approximated by straight lines and their intercepts with the lines of initial values. These yield a critical H-concentration for the onset of buckling. b) Comparison of critical concentrations determined optically and from stress curves (2\% deviation). c) The relation between hydrogen concentration and film thickness for $20 \%$ fraction of detached area is shown.

As shown in the Fig. 3.7(a), with increasing hydrogen concentration the detached film area increases steadily after the onset of buckling. The critical stresses for these critical concentrations can be determined. The stress values obtained in this way are included in Fig. 3.8. In this figure the critical stresses for delamination are plotted versus inverse square root of film thickness $\mathrm{d}$, as this relation represents Eq. (1.33). From the slope of the resulting curve the adhesion energy can be calculated. 


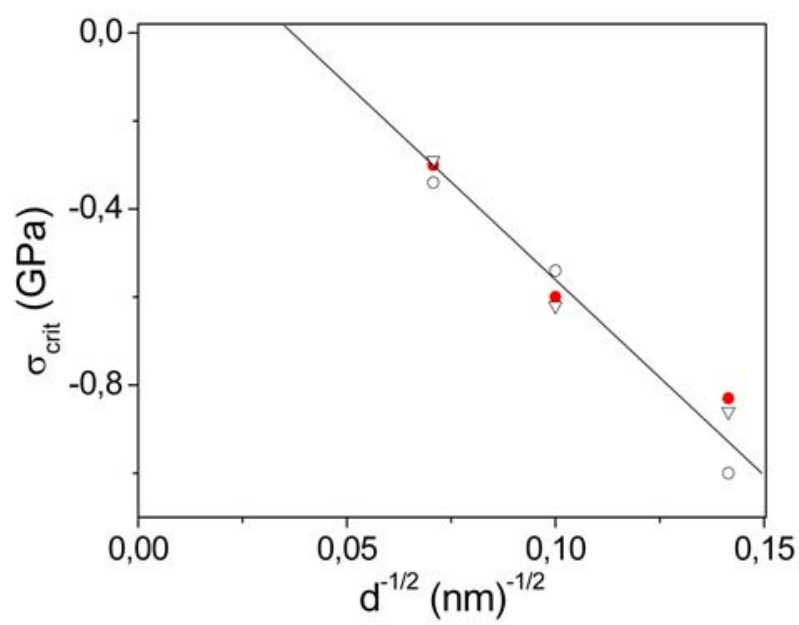

Figure 3.8: Critical stress for delamination of Pd (10 nm)/ Nb (50,100,200 nm) /Pd (10 nm) on $0.5 \mathrm{~mm}$ and $1 \mathrm{~mm} P C$ substrate. The critical stresses for buckling are obtained from stress measurements as presented in Fig. 3.1 and plotted versus the inverse square root of film thickness $d$. (Closed circles: 0.5 mm PC, open circles: $1 \mathrm{~mm}$ PC). The triangles are obtained from light microscopy investigations of the critical hydrogen concentration $\mathrm{H} / \mathrm{Nb}$.

Summarizing the measurements of critical stresses and hydrogen concentrations for films with different film thicknesses it can be pointed out that thinner films require larger critical stress and higher hydrogen concentration for buckling. This result is in accordance with Eq. (1.31), which shows that more elastic energy can be stored in thicker films. The influence of Pd top layer on the critical hydrogen concentration is not significant because of the much higher stresses for buckling than the tensile stress for stretching the Pd layer. However, it should influence the buckle height. This effect of Pd top layer will be discussed in Chap. 3.4.4 by buckle geometry investigations using the white-light interferometer.

\subsubsection{Substrate thickness impact}

The experimental results for the critical hydrogen concentrations determined by loading of samples on substrates with different thicknesses, as they are shown in Figs. 3.3, 3.4 and 3.5 are summarized in Fig. 3.9. All critical concentrations obtained for films on thinner substrates are significantly larger than those obtained for films on thicker substrates. Therefore, it is reasonable to assume that the thin substrate is slightly strained by the film during hydrogen loading. 
a)

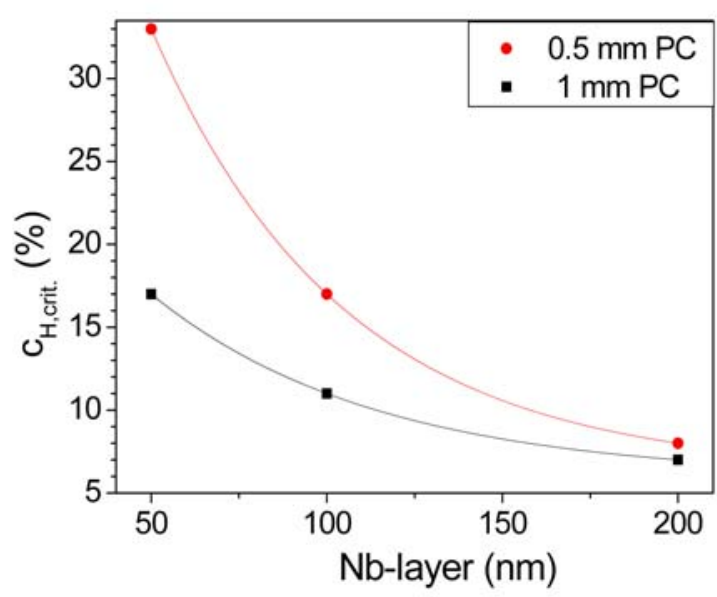

b)

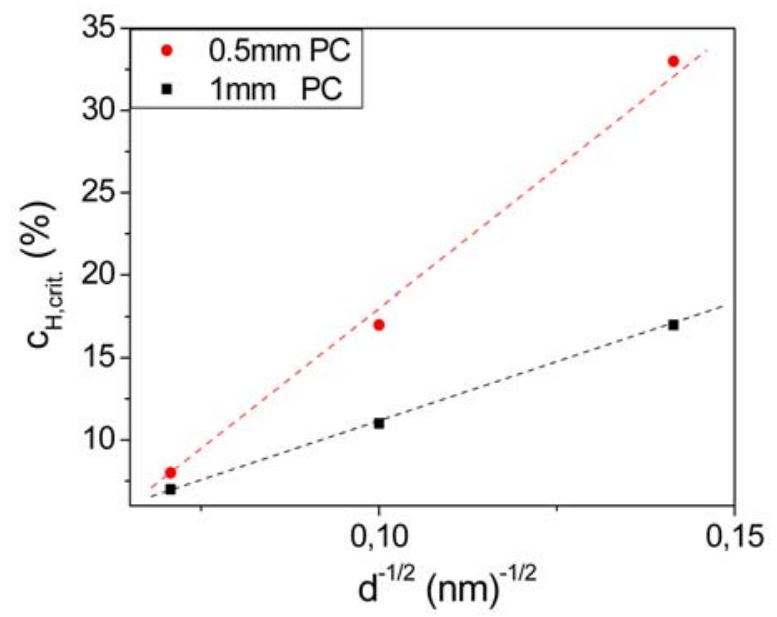

Figure 3.9: Critical hydrogen concentrations for the onset of buckling in $P d / \mathrm{Nb} / \mathrm{Pd}$ films on $1 \mathrm{~mm}$ and $0.5 \mathrm{~mm}$ PC as a function of a) film thickness, b) inverse square root of film thickness. The increase of the critical hydrogen concentration with decreasing $\mathrm{Nb}$ thickness can be seen. The linear relation of $c_{c r i t}$ and $d^{-1 / 2}$ confirms the theoretical prediction in Chap. 1.2.4.

In order to understand the lowering of the critical hydrogen concentration with increasing substrate thickness, hydrogen loading of a $\mathrm{Pd} / \mathrm{Nb} / \mathrm{Pd}$ film on very thin $0.02 \mathrm{~mm}$ PC was performed at HASYLAB. The results are shown in the Fig. 3.10.

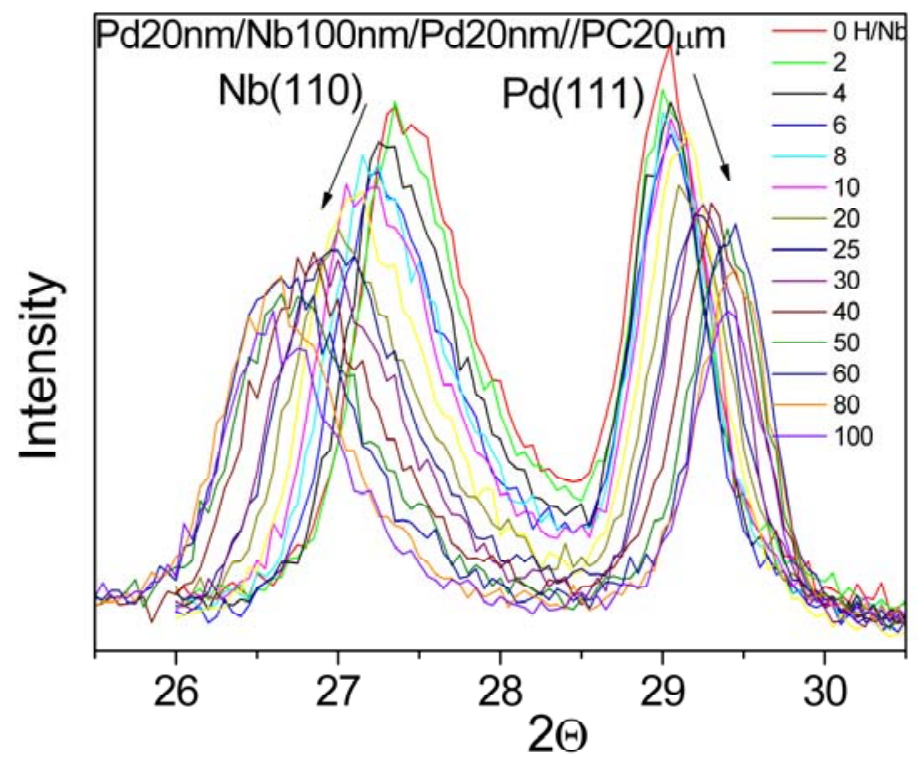

Figure 3.10: $\quad X R D$ spectra of $P d(20 \mathrm{~nm}) / \mathrm{Nb}(100 \mathrm{~nm}) / \mathrm{Pd}(20 \mathrm{~nm}) / / \mathrm{PC}(20 \mu \mathrm{m})$ film measured during hydrogen loading. A shift of the Pd(111) peak to larger angles signifies the lateral stretching of the Pd layer and, at the same time, the stretching of the substrate since the metal film is bonded to the substrate, but does not absorb hydrogen. 
It can be seen that the Pd (111) peak shifts to higher diffraction angels during hydrogen loading. This manifests a decreasing out-of-plane lattice spacing, as it follows from the stretching of the palladium lattice due to in-plane expansion. Thereby, the lateral expansion of the Pd-layer is due to the lateral expansion of Nb-layer, because hydrogen absorbs predominantly into the $\mathrm{Nb}$ layer. Since the film is bonded to the substrate that means stretching of the substrate as well. This observation is sketched in Fig. 3.11, where the expansion behavior of a $\mathrm{Pd} / \mathrm{Nb} / \mathrm{Pd}$ film during hydrogen loading on a hard (a) and on a flexible (b) substrate is illustrated.

a)
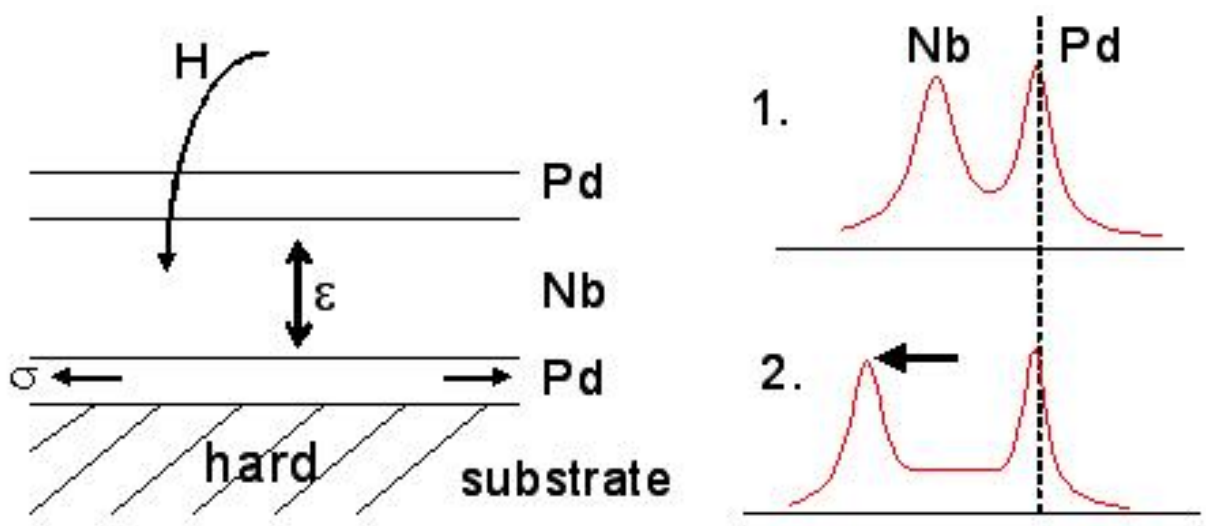

b)

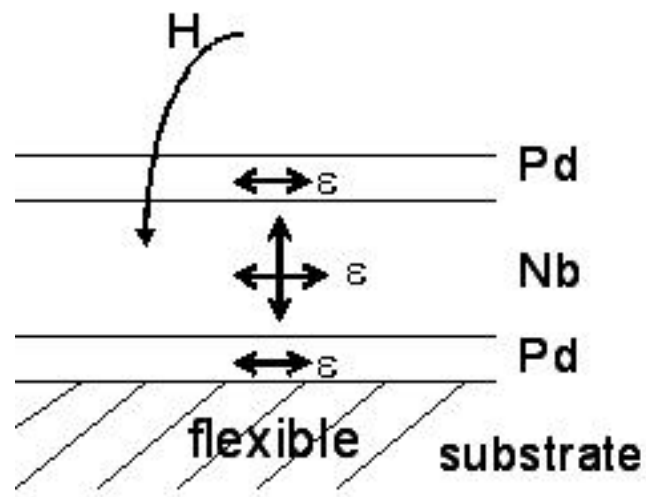

1.

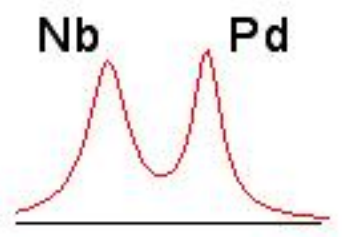

2.

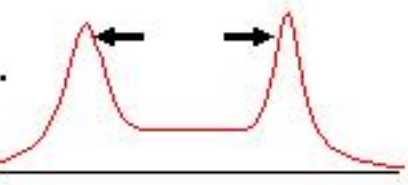

Figure 3.11: Schematic picture of hydrogen loading and XRD scattering of Pd/Nb/Pd films on a) hard and b) flexible substrates. The direction of strains in different layers of a metal film by hydrogen loading due to the different hydrogen solubility is shown. The changes in the XRD scattering figure of $\mathrm{Nb}$ (110) and Pd (111) before (1) and during (2) hydrogen loading are also shown.

In Fig. 3.12 the relative changes of the lattice constant $\left(a-a_{0}\right) / a_{0}$ are given, with the initial values without hydrogen $a_{0}$. 


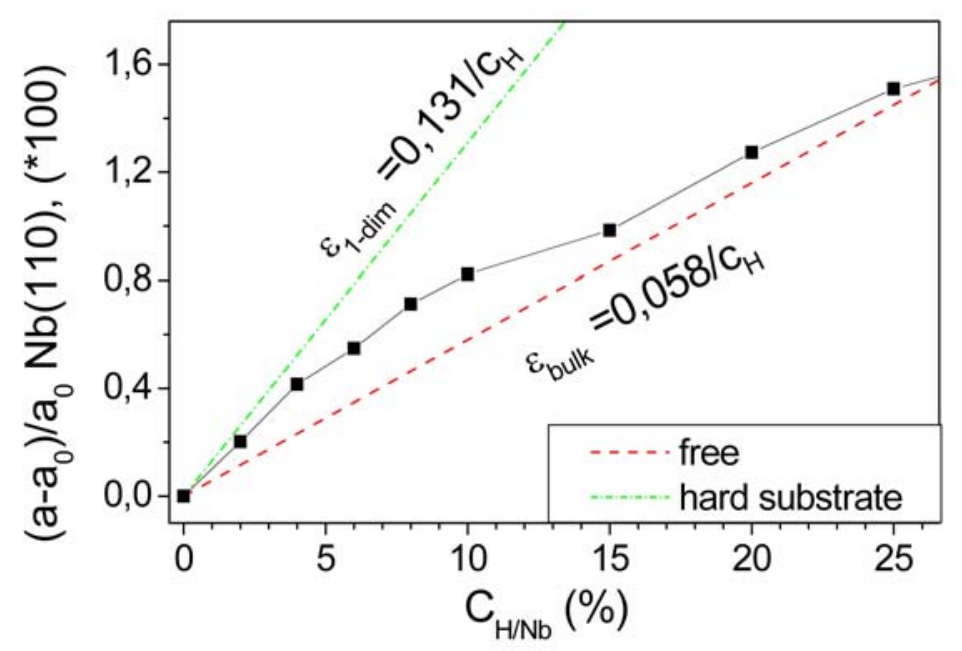

Figure 3.12: Relative changes of the interplanar spacing of $N b(110)$ vs. $H$ content in $P d / N b(100$ $\mathrm{nm}) / \mathrm{Pd} / \mathrm{PC}(0.02 \mathrm{~mm})$ film for low concentrations. The development of the curve up to $4 \% \mathrm{H} / \mathrm{Nb}$ is in good accordance with the theoretical calculations for expansion of a Nb-layer which is ideally bonded to its substrate. Large deviations at higher hydrogen concentrations can be seen.

It can be seen that the lattice constant increases linearly for the first two loading steps. The values in these two steps are close to the values for a Nb-layer that is ideally bonded to a hard substrate (see figure). The further development of this curve shows the large deviation from the lattice expansion of $\mathrm{Nb}$ films on hard substrates.

In Fig. 3.13 the emf of $\operatorname{Pd}(20 \mathrm{~nm}) / \mathrm{Nb}(100 \mathrm{~nm}) / \mathrm{Pd}(20 \mathrm{~nm}) / / \mathrm{PC}(20 \mu \mathrm{m})$ film by hydrogen loading is compared to the emf measurement on $\mathrm{Pd}(20 \mathrm{~nm}) / \mathrm{Nb}(1950 \mathrm{~nm}) / / \mathrm{Al}_{2} \mathrm{O}_{3}$. The decrease of the slope of the emf versus $\log c$ curve indicates the onset of the two phase region, which is for the $1.95 \mu \mathrm{m} \mathrm{Nb}$ film very similar to the bulk values. The solid solution limit for the film on PC is slightly increased due to the initial stress of this film. The $(\alpha+\beta) / \beta$-phase boundary is at larger hydrogen concentration in the film on PC compared to the film on sapphire what can be due to hydrogen loss in the film on PC. A higher stress during hydrogen loading in the film on sapphire also leads to smaller hydrogen concentrations for the completion of $\beta$-phase in comparison to the $100 \mathrm{~nm}$ niobium film on $20 \mu \mathrm{m}$ PC. 


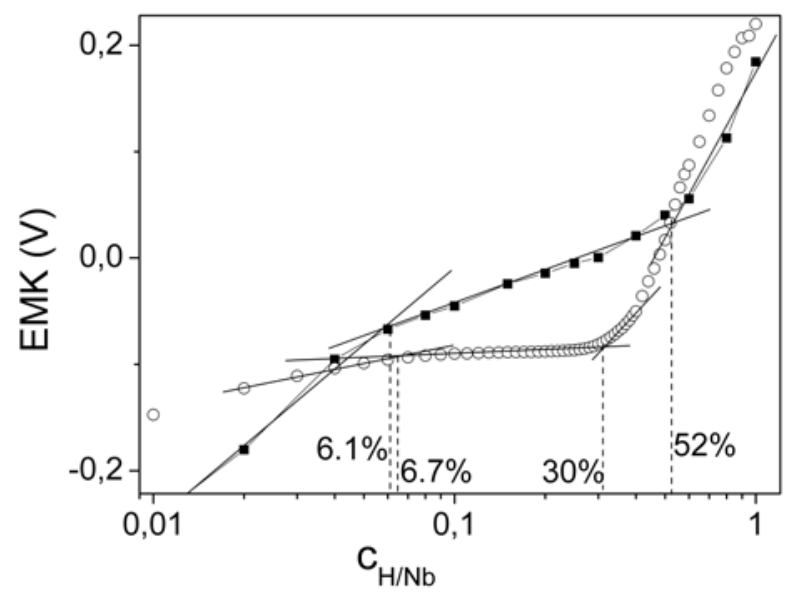

Figure 3.13: Emf of Pd(20nm)/Nb(100nm)/Pd(20nm)//PC(20 $\mu \mathrm{m})$ film (black squares) as a function of hydrogen concentrations; $\mathrm{Emf}$ of $\mathrm{Pd}(10 \mathrm{~nm}) / \mathrm{Nb}(1.95 \mu \mathrm{m}) / / \mathrm{Al}_{2} \mathrm{O}_{3}(0.2 \mathrm{~mm})$ (opened circles), (Ag/AgCl/Cl ${ }^{-}$electrode).

In Figs. 3.14 and 3.15 the relative change of the $\mathrm{Nb}(110)$ and $\mathrm{Pd}(111)$ spacing divided by $\mathrm{c}_{\mathrm{H} / \mathrm{Nb}}$ is plotted versus $\mathrm{c}_{\mathrm{H} / \mathrm{Nb}}$ for $100 \mathrm{~nm} \mathrm{Nb}$ film with $\mathrm{Pd}$ top- and underlayer. Since the buckling process leads to stress relaxation in the metal film the relative change of the lattice parameters should be smaller at, the onset of buckling. The proportionality-factor $k$ of the relative change of $\mathrm{Nb}(110)$ increases during the first two loading steps, shows a maximum at about 0.00075 for 6$10 \% \mathrm{H} / \mathrm{Nb}$, and then steadily decreases. The decrease of $k$ correlates with the onset of hydrideformation, as it was proven by the emf measurement shown in Fig. 3.13 and optical surface observation of $\mathrm{Nb}$ films with thicknesses $1.95 \mu \mathrm{m}$ and larger in Chap. 3.5.

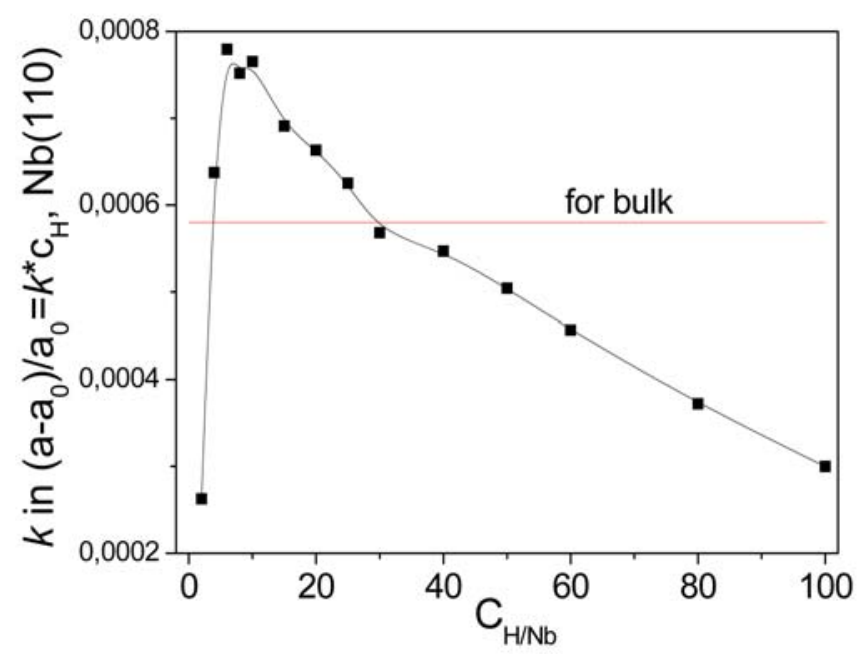

Figure 3.14: Correlation between the relative change of the $N b(110)$ lattice spacing and hydrogen concentration for $100 \mathrm{~nm} \mathrm{Nb}$ film on $20 \mu \mathrm{m} \mathrm{PC}$. 


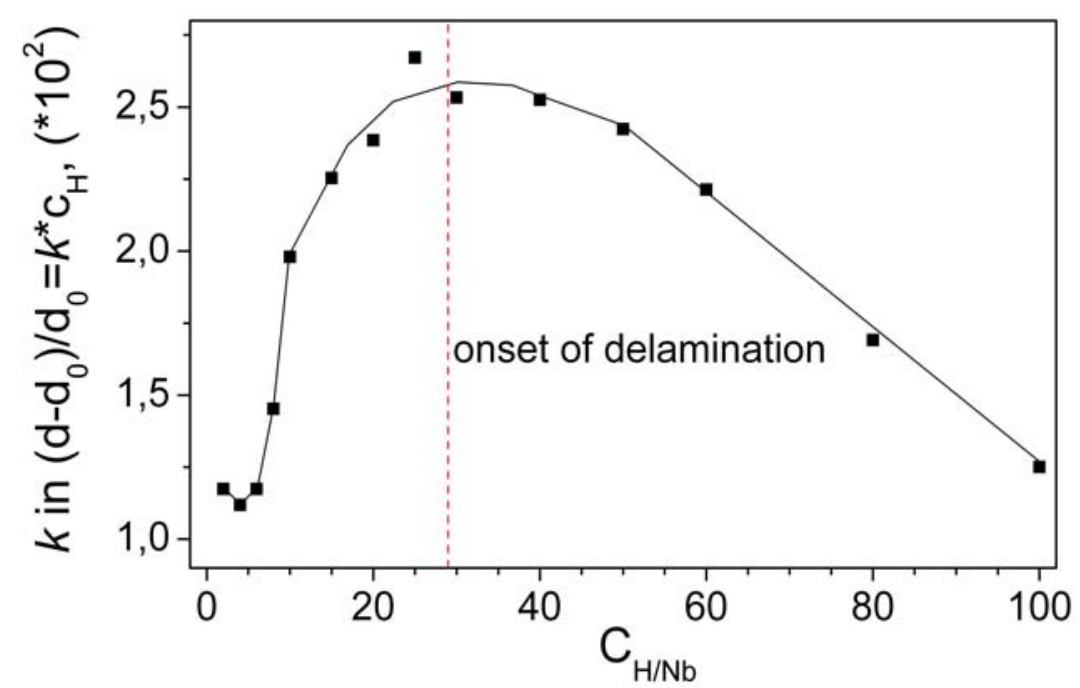

Figure 3.15: Correlation between the relative change of the Pd(111) lattice spacing and hydrogen concentration for $100 \mathrm{~nm} \mathrm{Nb}$ film on20 $\mu \mathrm{m} \mathrm{PC}$.

The decrease of Pd-layer expansion in Fig. 3.15 can be correlated with the onset of buckling. The main parts of delaminated film areas are no longer parallel to the sample surface. Therefore, they are not detected by XRD, as it is explained in the discussion of Fig. 3.16. The decrease of this curve is referred to the stress relaxation in the not-delaminated areas during film buckling. Therefore, from the curve maximum the critical concentration is about $25 \% \mathrm{H} / \mathrm{Nb}$.

To summarize, the hydrogen loaded $\mathrm{Pd} / \mathrm{Nb} / \mathrm{Pd}$ films on thin Polycarbonate substrates do not only expand in vertical direction, as it was expected for a strongly clamped film on a rigid substrate, but they do also expand laterally. This lateral expansion is accompanied by a lateral expansion of the substrate. This extension leads to a reduction of elastic energy in the film and to an increasing critical hydrogen concentration for delamination.

In situ XRD observation of hydrogen loaded $\mathrm{Pd} / \mathrm{Nb} / \mathrm{Pd}$ films on $1 \mathrm{~mm} \mathrm{PC}$ was performed at HASYLAB. The results are shown in Fig. 3.16. The film was buckled very strongly. Therefore it can be seen that the diffracted X-ray intensity strongly decreases, and it was not possible to detect the $\mathrm{Nb}$-hydride phase transition during this measurement. At the end of the measurement the film was not intact in the region of the incident beam any longer. 


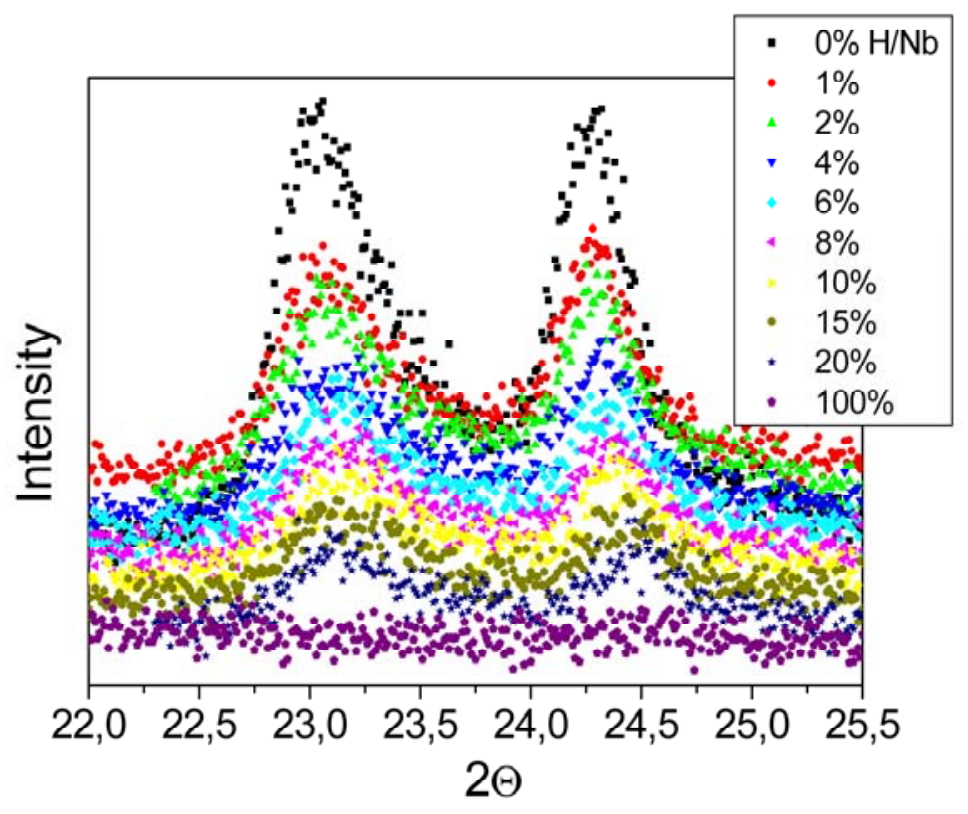

Figure 3.16: XRD measurements performed at HASYLAB of a Pd(10nm)/Nb(200 $\mathrm{nm}) / \mathrm{Pd}(10 \mathrm{~nm}) / / \mathrm{PC}(1 \mathrm{~mm})$. The intensity decreases because of very strong buckling in the region of the $X$-ray incident beam was observed

Summarizing the results obtained for critical stresses and concentrations for films on substrates with different thickness it can be concluded that the critical hydrogen concentrations for films on thicker substrates are significantly smaller. But the critical stresses for the films on substrates with different thickness are similar, as it was shown in chapters 3.1.2 and 3.1.3. The agreement of the critical stresses derived analyzing the deviations of the measured compressive stress from the linear stress evolution and from the fraction of the delaminated film areas show indeed, that these deviations are caused by buckling. 


\subsubsection{Continuous hydrogen loading}

In order to be able to quickly perform adhesion measurements using the hydrogen loading method it was decided to investigate continuous hydrogen loading instead of stepwise loading, as well. Thereby the continuous loading does not only allow fast measurements, but it also allows a fast mathematical analysis of the results. First, continuous hydrogen loading of 3 samples with $\mathrm{Pd} / \mathrm{Nb} / \mathrm{Pd}$ on $1 \mathrm{~mm} \mathrm{PC}$ with $200 \mathrm{~nm} \mathrm{Nb}$-layer and $10 \mathrm{~nm} \mathrm{Pd}$ coping shall be described. The density of the loading current was $0.154 \mathrm{~mA} / \mathrm{cm}^{2}$, so that the time needed for hydrogen loading from 0 to $100 \% \mathrm{H} / \mathrm{Nb}$ was about $19 \mathrm{~min}$ for one sample. The applied current density was approximately two times smaller than that applied during stepwise loading. Such a smaller current density allow a complete absorption and distribution of hydrogen atoms in the sample, even one does not wait for equilibrium after a certain loading step. The complete hydrogen absorption can be assumed, because no hydrogen gas bubbles were observed on the sample surface during this loading. The stress curves for the first hydrogen loading of the three samples can be seen in Fig. 3.17. These samples obtain the same structural film parameters, as they were manufactured in the same preparation run. While one sample exhibits a critical stress of $c_{\text {crit }}=-1.2$ $\mathrm{GPa}$, the value of another sample reaches (only) $40 \%$ of this value. At the same time it can be seen clearly that the same critical concentration values appear. The critical stress value seems to depend strongly on the sample. This stress behaviour was unexpected and required clarification.
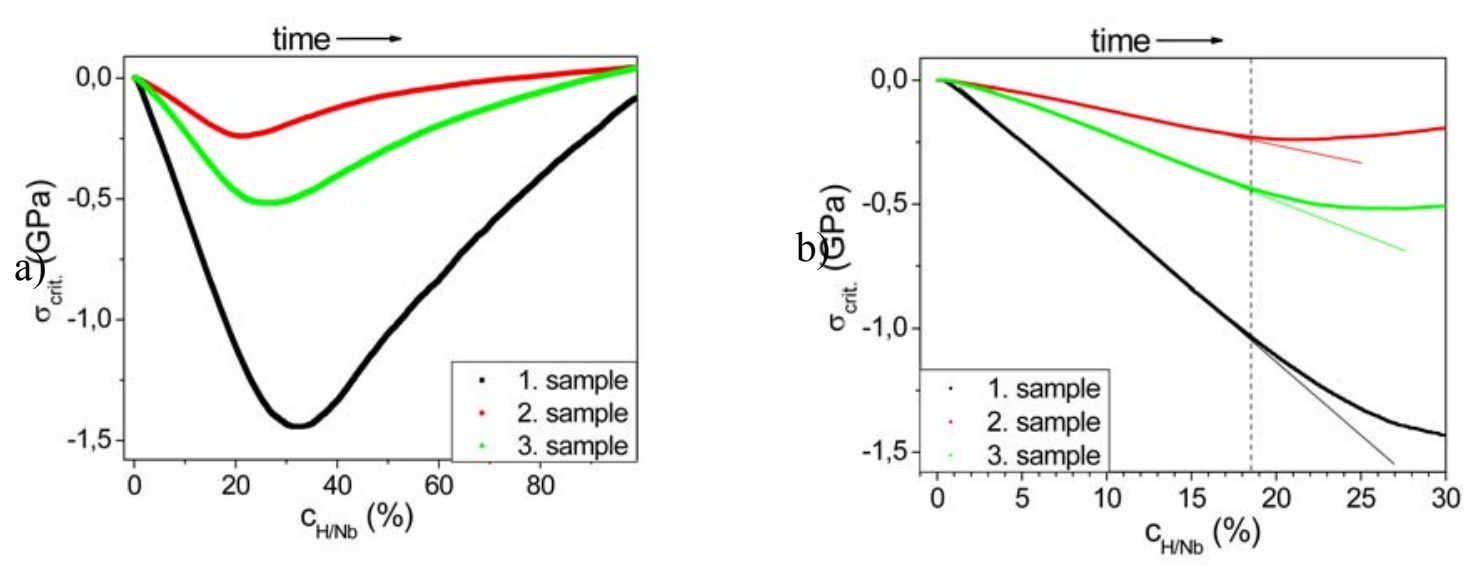

Figure 3.17: Compressive stresses during continuous electrochemical hydrogen loading of three Pd (10 $\mathrm{nm}) / \mathrm{Nb}(200 \mathrm{~nm}) / \mathrm{Pd}(10 \mathrm{~nm}) / / \mathrm{PC}(1 \mathrm{~mm})$ samples prepared during the same deposition run. The critical concentration (proportional to the indicated time) is constant, while the critical stress is strongly changed. Possible reasons for these deviations are discussed in the text; a) concentration range of 0$100 a t \% \mathrm{H} / \mathrm{Nb}$, b) concentration range of $0-30 a t \% \mathrm{H} / \mathrm{Nb}$; the critical hydrogen concentration for delamination is shown by the dashed line.

Some possible reasons for this effect were found: 1) The polymer is relaxing under the detached area. Light microscopy imaging shows a definite optical change of the PC surface in the 
field of original wrinkles after removing the buckled metal layer. The optical changes on the substrate surface represent the buckle areas. More details on this effect are described in Chap. 3.2. 2) The bending method is a one-point-measurement with an inductive sensor. Changes of the sample inclination in the mounting plate cannot be separated from changes in the sample curvature, while it is not possible to mount each sample exactly the same way. Of course these deviations affect the calculated mechanical stresses. To optimize the measuring conditions, the length of the clamped part of the samples was chosen to be not too short, e.g. some millimetres and the samples were clamped with enough force in order to avoid slipping of the sample in the holder during bending. Further, length to width ratio of all the samples was kept constant.

A critical concentration of about 18.5 at $\% \mathrm{H} / \mathrm{Nb}$ for all three samples was found setting the threshold value to be $2 \%$ deviation of the stress curve from linear behavior. This critical concentration is larger than expected. The reason for this effect can be assumed to originate from the larger loading current density. Therefore, the dependence of the critical hydrogen concentration on the current density was examined. It is assumed that the critical hydrogen concentrations are larger for a higher current density by two reasons. On the one hand, it is expected that during fast loading not all hydrogen atoms can be absorbed by the film, leading to hydrogen losses and therefore to a larger nominal critical hydrogen concentration. On the other hand, in case of hydrogen loading with a higher current density hydrogen is enriched in the upper layer of niobium due to diffusion limitations. This effect courses an inhomogeneous stress distribution in the niobium layer and a higher hydrogen concentration for buckling. The approximation of the hydrogen concentration at the interface to the substrate was made by assumption of hydride formation in the upper layer of the film with the thickness $d_{1}$ as shown in Fig. 3.18.

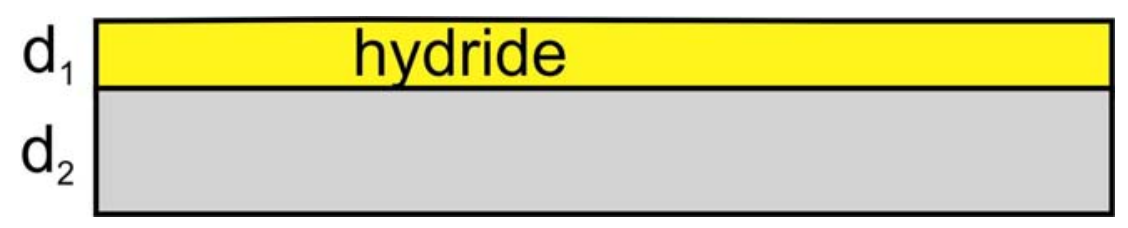

Figure 3.18: Schematical drawing of a sample with hydride formed in the upper layer of thickness $d_{1}$. Assuming that the hydrogen concentration in the top $\mathrm{Nb}$ layer is $c_{1}=60 \%$ (hydride) with a thickness of $\mathrm{d}_{1}=20 \mathrm{~nm}$ and in the underlayer with a thickness $d_{2}=80 \mathrm{~nm}$ the hydrogen concentration is $c_{2}=11 \%$ (expected critical concentration), then the total hydrogen concentration in the metal film is 


$$
c_{H}=\frac{d_{1} \cdot c_{1}+d_{2} \cdot c_{2}}{d_{1}+d_{2}}=20.8 \%
$$

This model explains the higher critical concentration obtained in these measurements.

Each sample was loaded three times, and the results are shown in Fig. 3.19. The loading cycles were performed under the same conditions as described above.

a)

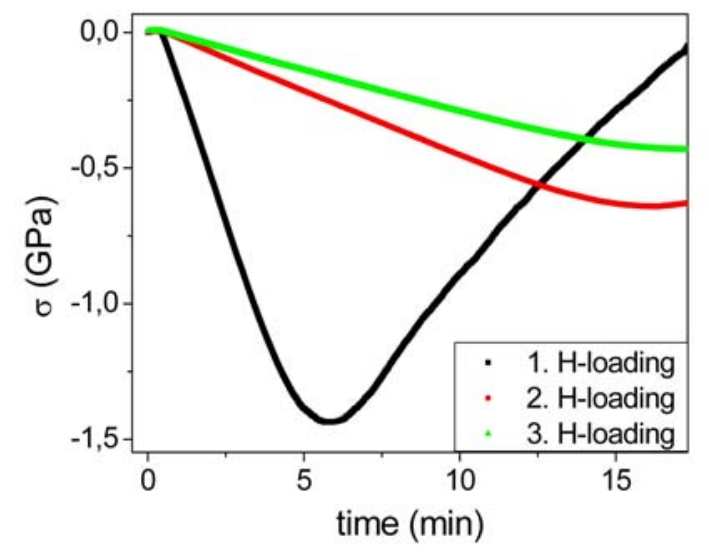

b)

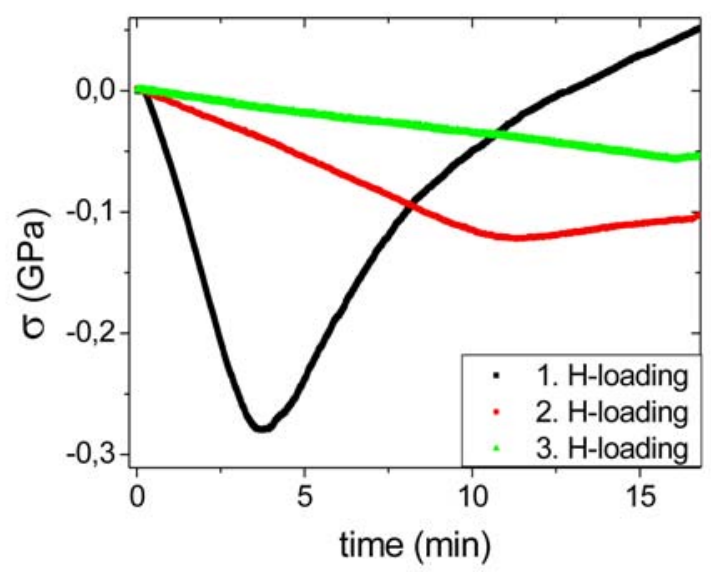

c)

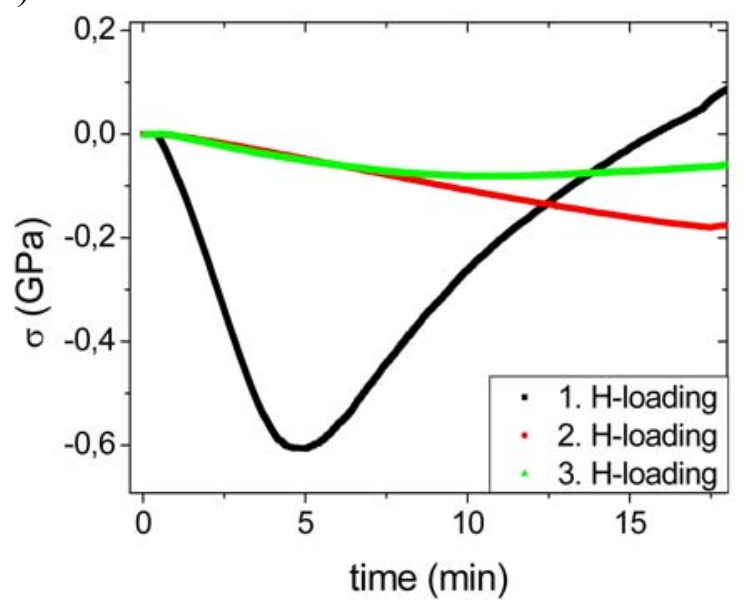

Figure 3.19: H-loading cycles of three Pd/Nb/Pd// $1 \mathrm{~mm}$ PC samples (with $20 \mathrm{~nm} / 200 \mathrm{~nm} / 20 \mathrm{~nm}$ thickness layer respectively) deposited at the same conditions. The stress slope is smaller and the stress relaxation occurs at larger hydrogen concentrations for the second and third hydrogen loadings. The samples are numerated as a), b) and c).

The unloading was performed by applying $0.4 \mathrm{~V}$ voltage for 2 hours. Obviously in each further loading cycle the sample bending is reduced. This effect can be explained by the increase of the delaminated area, reducing the stress development in the samples. Therefore, the critical concentration for the second and third loading is larger than for the first loading, as the critical stress, necessary for the delamination of further film fractions, is reached at higher hydrogen 
concentrations. For clarity, the stored elastic energy is modulated by the existence of buckled and unbuckled film fractions.

To study the impact of the PC-surface conditions on the adhesion, continuous hydrogen loading of $\mathrm{Pd} / \mathrm{Nb} / \mathrm{Pd}$ films with $100 \mathrm{~nm} \mathrm{Nb}$-layer and 10nm Pd layers on $1 \mathrm{~mm}$ differently pretreated PC-substrates was performed. The PC used in this study was protected by a plastic film on its surface. After removal of the film two of the four samples were cleaned by dipping it in isopropanol in an ultrasonic bath for $30 \mathrm{sec}$. The other two were not pre-treated before film deposition. Four different samples were investigated, while each two of these samples with differently pre-treated PC have been prepared in the same deposition run.

The samples were continuously electrochemically charged with hydrogen at a current density $\mathrm{j}$ $=0.0765 \mathrm{~mA} / \mathrm{cm}^{2}$ up to $40 \%$ of hydrogen in niobium. In Fig. 3.20 the stress evolution in the samples during H-loading is shown. It can be seen that the compressive stress increases linearly at the beginning for all samples, but that the initial slopes and the concentration range of compressive stress increase are larger for the samples with the isopropanol-treated surfaces. The deviation from the linear stress increase starts reproducibly at about $18-19 \% \mathrm{H} / \mathrm{Nb}$ for the cleaned samples and about $12.5 \% \mathrm{H} / \mathrm{Nb}$ for the others. The critical stress is also larger for the samples with cleaned surfaces.

a)

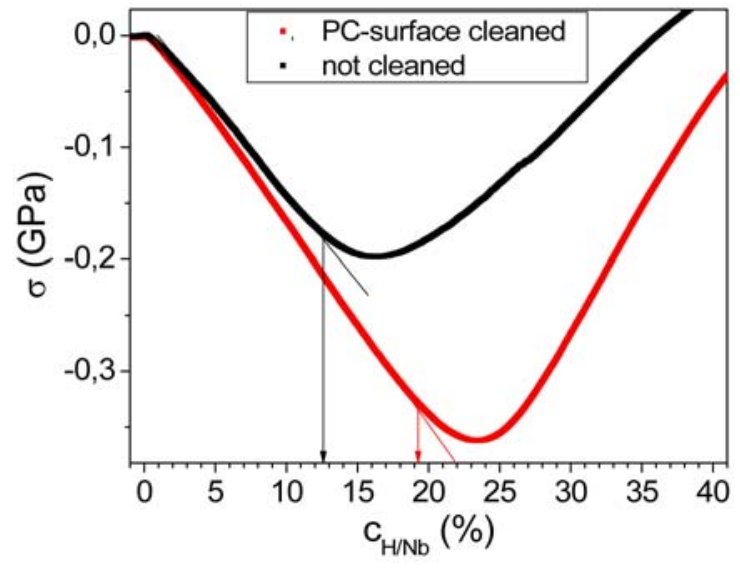

b)

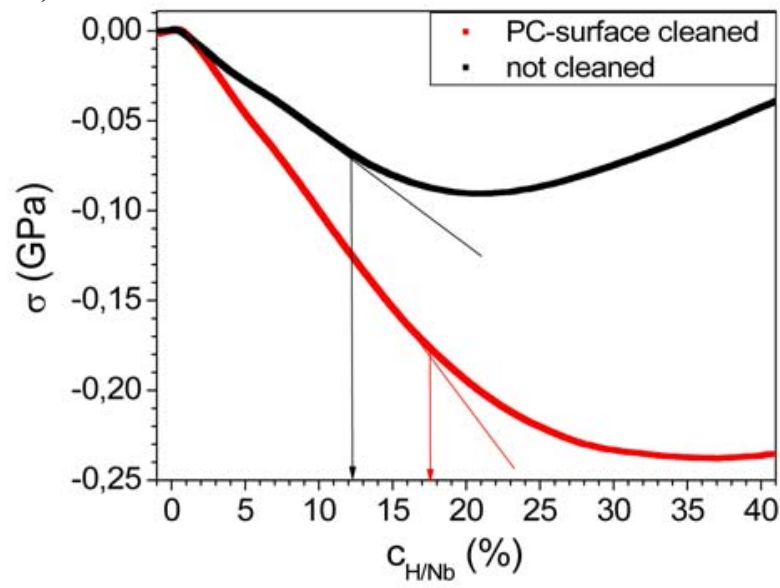

Figure 3.20: Stress vs. concentration of hydrogen by H-loading for a) first two samples b) second two samples. The stress slopes and the critical hydrogen concentrations are larger for the films sputtered on the isopropanol-cleaned PC surfaces. The buckles formation in the second two samples was not so intensive like in the first two samples what can be seen in the relaxation behavior.

The relaxation behavior of the first two samples (see Fig 3.20a), which is accompanied by the buckling process, is stronger than for the second two samples (Fig. 3.20b). Fewer amounts of buckles after H-loading in the second two samples was also seen compared to the first two samples. This is in agreement with the stress curves in Fig. 3.20(b). 
In Fig. 3.21 the emf f $^{*}$ evolution of the first two samples (stress curve is shown in Fig. 3.20a) is compared to the bending data, obtained from the inductive sensor. The emf ${ }^{*}$ represents change of the chemical potential and was measured during continuous H-loading. Since the electrical potential is kept constant, changes are attributed to a change in the chemical potential. The same is shown in Fig. 3.22 for the second two samples (stress evolution is shown in Fig. 3.20b).

a)

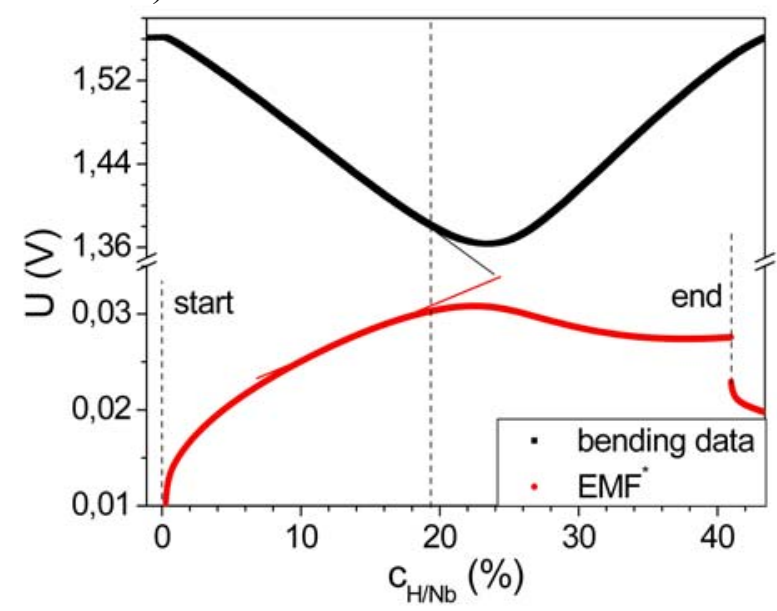

b)

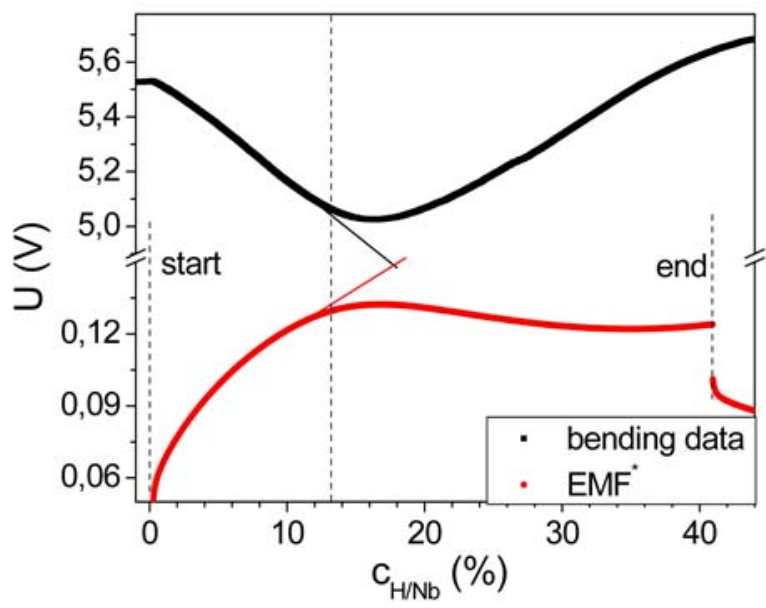

Figure 3.21: Sample bending vs. concentration of hydrogen by H-loading for the first two samples with a) cleaned surface of PC and b) surface as manufactured.

a)

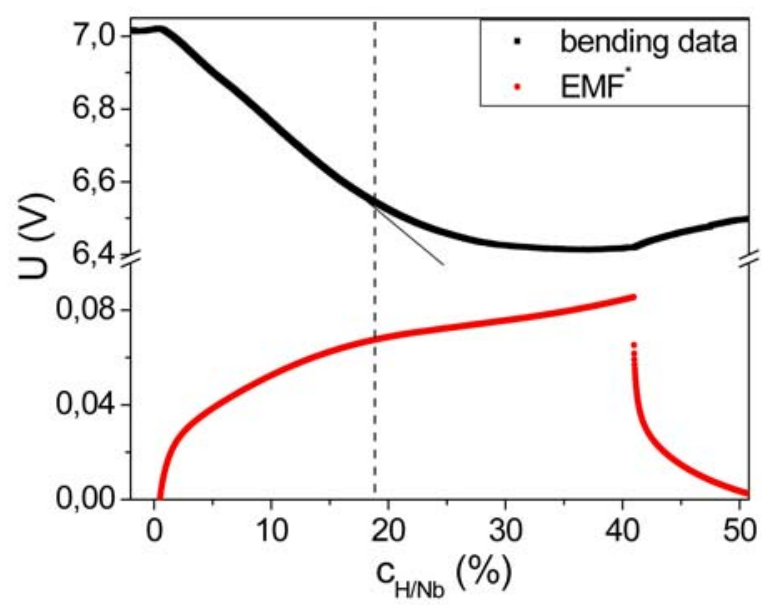

b)

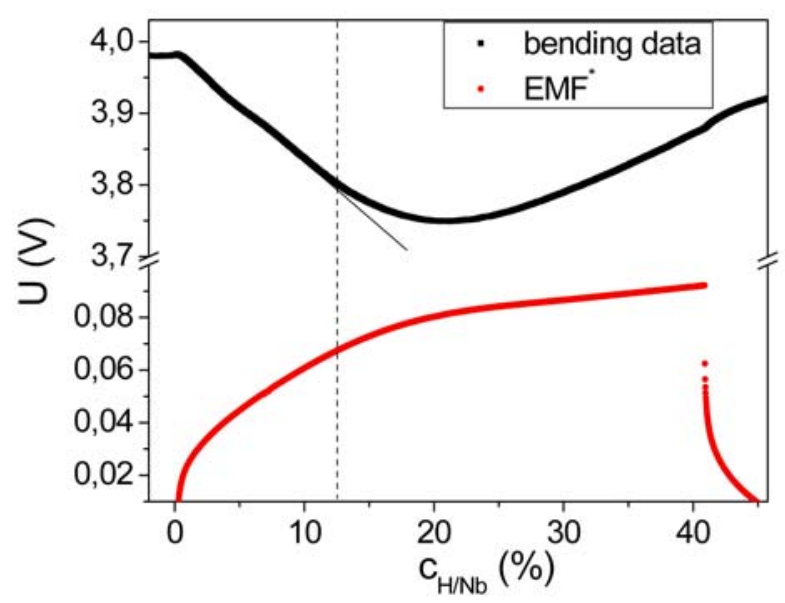

Figure 3.22: Sample bending vs. concentration of hydrogen by H-loading for the second two samples with a) cleaned surface of PC and b) surface as manufactured.

It can be pointed that for the first two samples the formation of buckles was more intensive than for the second two samples and that is also shown in the magnitude of the emf decrease for these samples, which is larger for more buckled films. The films on the PC surface cleaned with isopropanol show larger stress during hydrogen loading and higher critical hydrogen concentrations for buckling. In Chap. 3.4.2 adhesion energies of metal films on differently pre- 
treated PC will be compared. Thereby plasma pre-treated substrates and the substrates without pre-treatment will be discussed.

\subsubsection{Buckle propagation, buckle width}

To study the surface morphology development of the sample surfaces during hydrogen loading, the surfaces were optically monitored, using the loading setup shown in Fig. 2.1.

In. Fig. 3.23 the morphology change of the surface of a $\mathrm{Pd}(10 \mathrm{~nm}) / \mathrm{Nb}(100 \mathrm{~nm}) / \mathrm{Pd}(10 \mathrm{~nm}) / / \mathrm{PC}$ (1mm) sample during loading is shown.

a) 0 at $\% \mathrm{H} / \mathrm{Nb}$

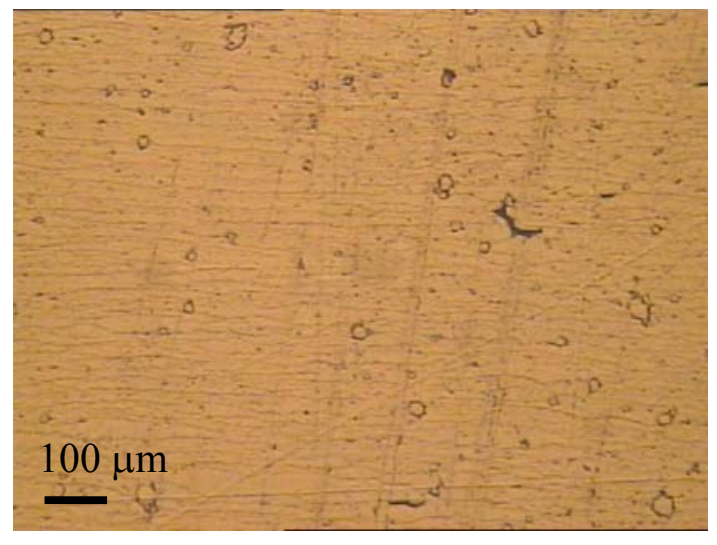

c) 15 at $\% \mathrm{H} / \mathrm{Nb}$

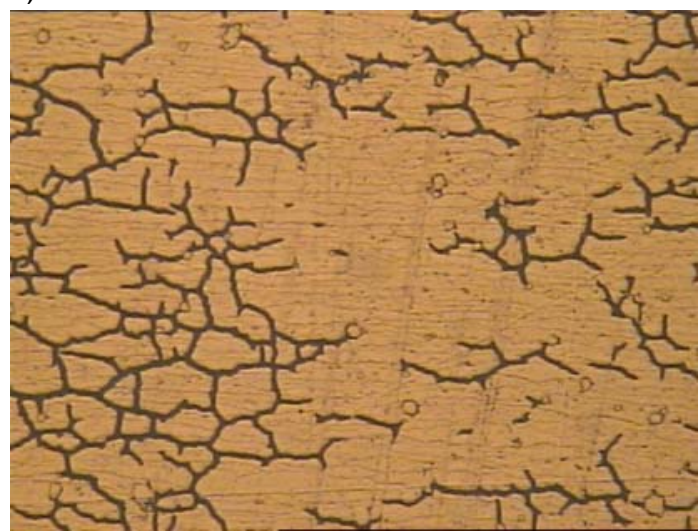

b) 11 at $\% \mathrm{H} / \mathrm{Nb}$

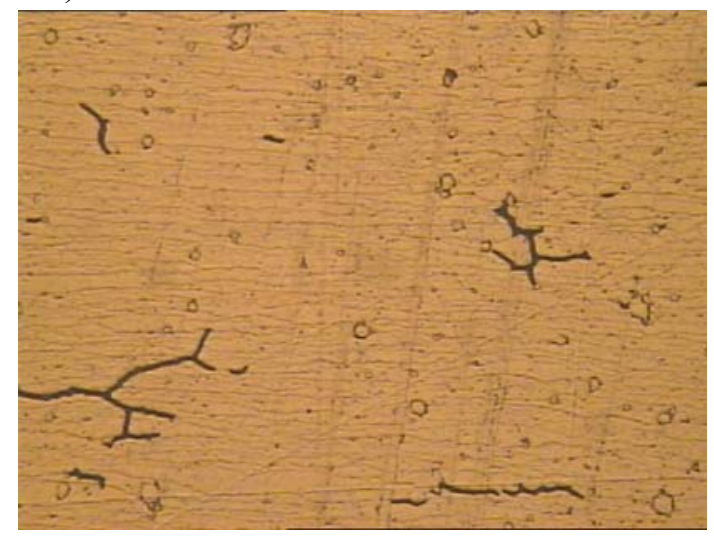

d) 20 at $\% \mathrm{H} / \mathrm{Nb}$

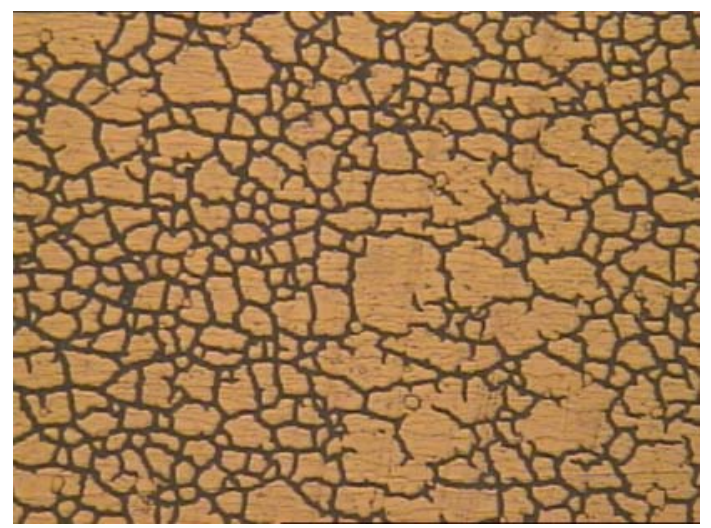

Figure 3.23: Surface of a Pd20nm/Nb100nm/Pd20nm film on $1 \mathrm{~mm}$ polycarbonate at different hydrogen concentrations. The growth of the network of buckles with increase of hydrogen concentration can be seen. The bar corresponds to a length of $100 \mu \mathrm{m}$.

It can be seen that there are already some buckles existing at the surface prior to hydrogen loading. These buckles were produced by the intrinsic stress in the film developing during film preparation. The growth of the existing buckles and the formation of new buckles until $11 \%$ $\mathrm{H} / \mathrm{Nb}$ is negligible, if one compares it to the further buckle evolution. At a hydrogen concentration larger than $11 \% \mathrm{H} / \mathrm{Nb}$, growth and formation of new buckles occurs abruptly. 
The images show that a large fraction of the sample does not delaminate from the substrate by exceeding the critical concentration. Therefore the question arises, if the adhesion energy varies locally or if there are other reasons for this observation. To answer this question the behaviour of the sample was examined at higher hydrogen concentrations. With increasing hydrogen concentration the observed segment is covered by a network of buckles. As can be seen in Fig. 3.24 the subsequent buckles propagation proceeds from already existing buckles by the consecutive formation of junctions. Interestingly the two arms of the junctions do not appear at the same time.

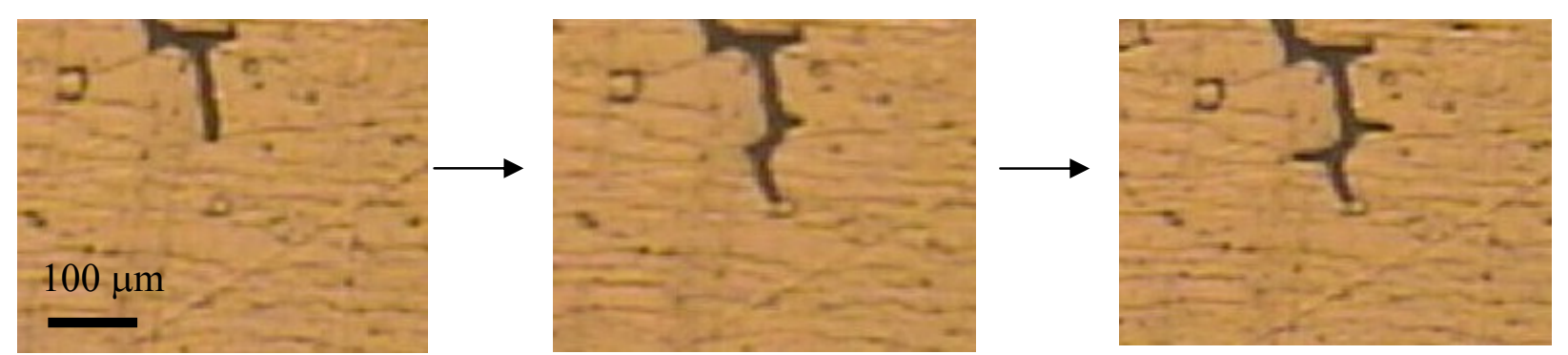

Figure 3.24: buckle growth of a Pd20nm/Nb100nm/Pd20nm film on $1 \mathrm{~mm}$ PC by growth of a new buckles perpendicular to the existing buckle and divided it on a half length.

Another mechanism of buckle growth was observed for a further sample during continuous hydrogen loading. As can be seen in Fig. 3.25 a buckle grows until a length of about three buckle widths is reached and then splits at the end into two new buckles, which grow with an angle of about $80-120^{\circ}$ to each other.
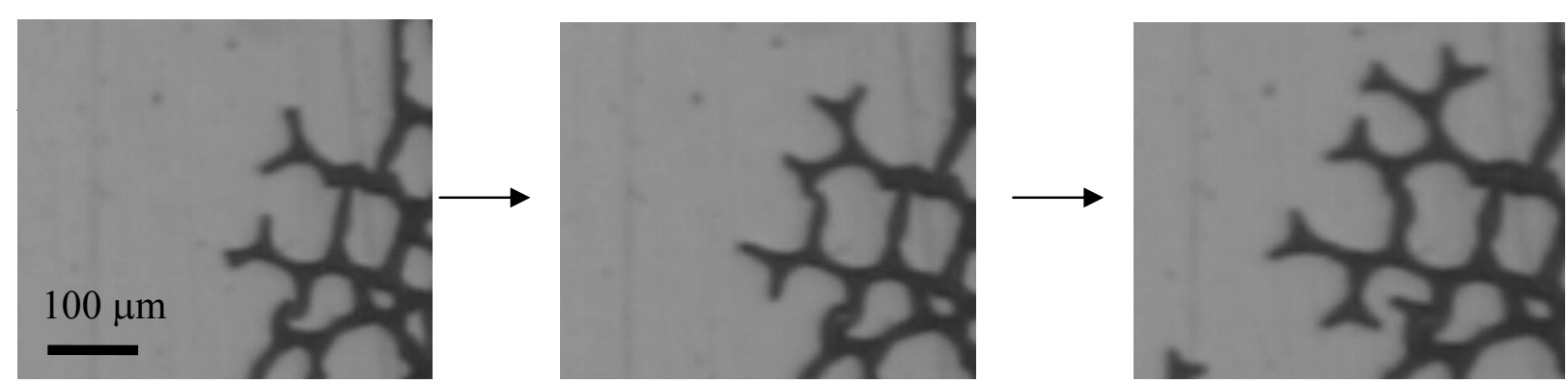

Figure 3.25: The mechanism of a buckle growth by splitting of the buckle end in two new buckles. The mechanism is repeated by the reach of the buckle characteristic length of about 3 times the buckle width.

The later buckle formation clearly depends on the already available buckle morphology. As the buckle morphologies appear to be quite regular, locally modified adhesion energy would not explain this pattern formation. It is suggested that the elastic energy increases homogeneously and is minimized by the film as a whole by local detachment. Probably these detached areas interact by elastic far fields, which are present in the film and in the substrate. 
It is to be expected that there exist small fluctuations in the film adhesion, so that the formation of wrinkles starts at sites of smallest adhesion energy. Since the buckle density rises very quickly, it can be assumed that an average adhesion energy value can be determined which is characteristic for a film-substrate-system.

It is also important to investigate the reproducibility of the critical parameters for experimentally similar prepared layer/substrate systems. Therefore a series of measurements was carried out, which shows that the values can be reproduced very well (see Fig. 3.48 in Chap. 3.4.1).

In case of $30-\mathrm{nm} \mathrm{Nb}$ films, a very dense buckle pattern occurs, which is shown in Fig. 3.26. In this figure the medium and the final state of detachment are displayed. By increasing the hydrogen concentration in the $30 \mathrm{~nm}$ film, the detachment pattern covers about $50 \%$ of the surface area in the maximum concentration state.
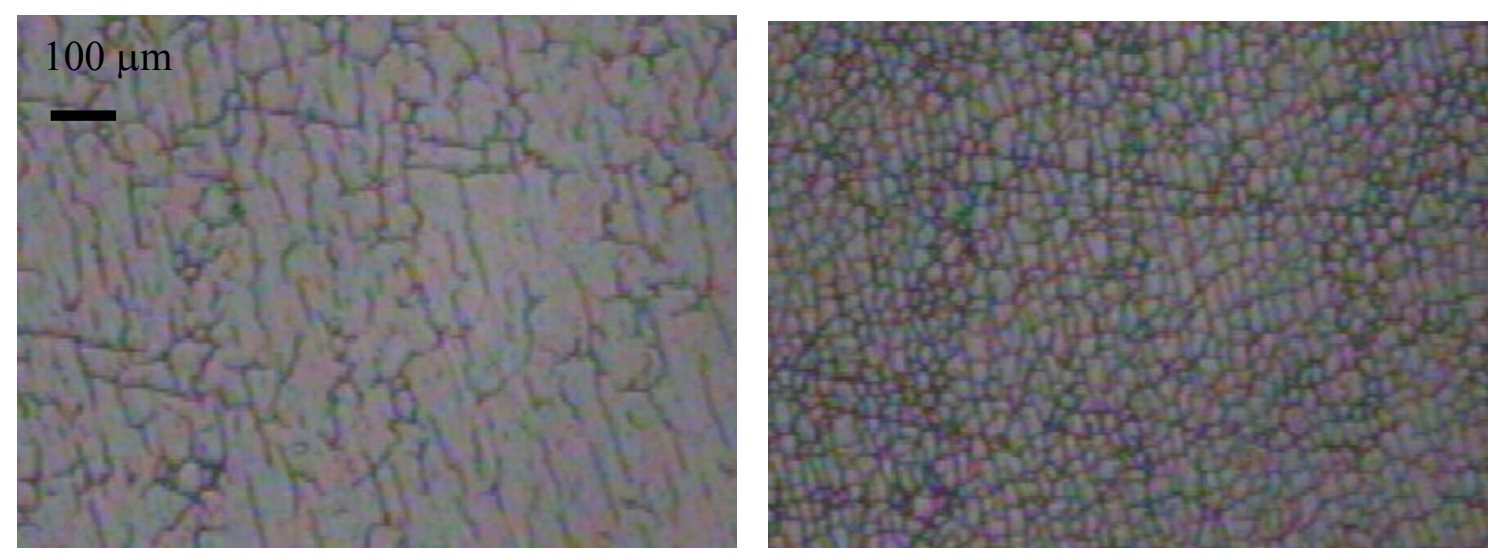

Figure 3.26: Delamination of a $30 \mathrm{~nm} \mathrm{Nb}$ film from $1 \mathrm{~mm} \mathrm{PC}$. The surface morphology at $70 \% \mathrm{H} / \mathrm{Nb}$ (left) and $135 \% \mathrm{H} / \mathrm{Nb}$ (right) is shown. The buckles morphology is analogue to the morphology of $\mathrm{Nb}$ films with larger thickness on PC. The buckles distribution is homogenous over the film surface.

The width of the buckles was measured during hydrogen loading of $\mathrm{Pd}(10 \mathrm{~nm}) / \mathrm{Nb}(100$ $\mathrm{nm}) / \mathrm{Pd}(10 \mathrm{~nm}) / / \mathrm{PC}(1 \mathrm{~mm})$ using light microscopy. A corresponding plot of buckle width versus hydrogen concentration is presented in Fig. 3.27. The critical hydrogen concentration for this film is $10 \%$ of $\mathrm{H} / \mathrm{Nb}$. 


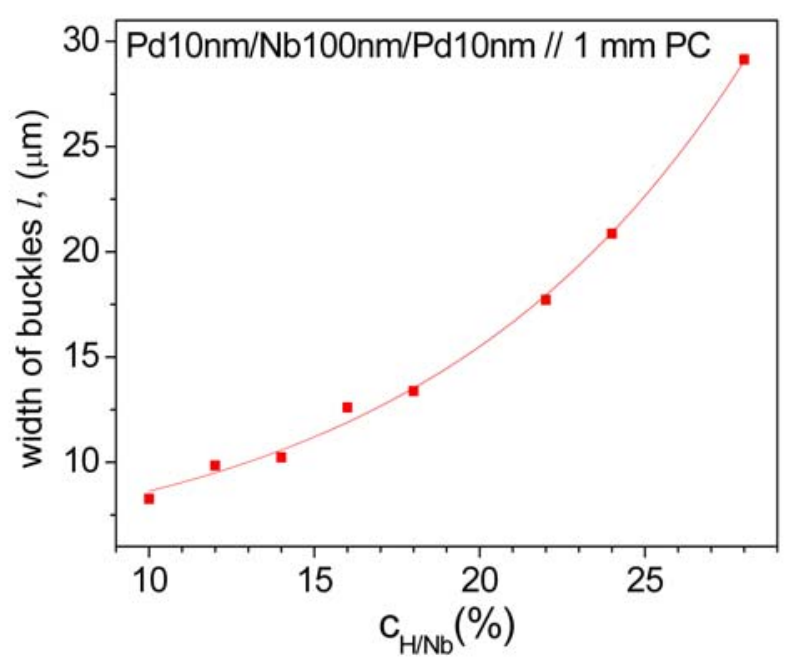

Figure 3.27: Buckles width development of Pd/Nb(100 nm)/Pd during H-loading. Buckle width growth with increasing hydrogen concentration was observed.

This figure shows an exponential increase of the buckle width. Theoretically, the increase of the buckle width is proportional to the square root of the stress, which is proportional to the hydrogen concentration. Thus, from Eq. 1.24

$$
l=\frac{l_{E u}}{\sqrt{\sigma_{E u}}} \sqrt{\sigma}
$$

where $l_{E u}$ is the initial buckle width at the critical Euler stress $\sigma_{E u}$. The stronger increase of the buckle width can be explained by the Gorsky effect. The diffusion of hydrogen atoms into the relaxed buckle regions results in a stress increase also in the buckles and supports their growth.

The initial width $l_{0}$ of the buckles for $\mathrm{Nb}$ films on $0.5 \mathrm{~mm}$ PC measured at the critical hydrogen concentration is compiled in Tab. 3.1. The average values determined by width measuring for buckles at different places of one image are given.

Table 3.1: Width of the buckles for niobium films on $0.5 \mathrm{~mm}$ polycarbonate at critical hydrogen concentration for buckling.

\begin{tabular}{|l|l|l|l|}
\hline Nb Thickness $d(\mathrm{~nm})$ & 50 & 100 & 200 \\
\hline Buckle Width $l_{0}=l_{E u}(\mu \mathrm{m})$ & 4.0 & 6.5 & 12.0 \\
\hline
\end{tabular}

The buckle width increases with increase of the film thickness. This is in a good agreement with the formula (1.23), which predicts that the width of a buckle is proportional to the film thickness and reciprocally proportional to the square root of the critical buckling stress, which is larger for thinner films. 
The relation between buckle width at critical hydrogen concentration and film thickness for $\mathrm{Pd} / \mathrm{Nb} / \mathrm{Pd}$ on 1 and $0.5 \mathrm{~mm} \mathrm{PC}$ is shown in Fig. 3.28. There the measured values are compared to the theoretical prediction by Euler's instability.

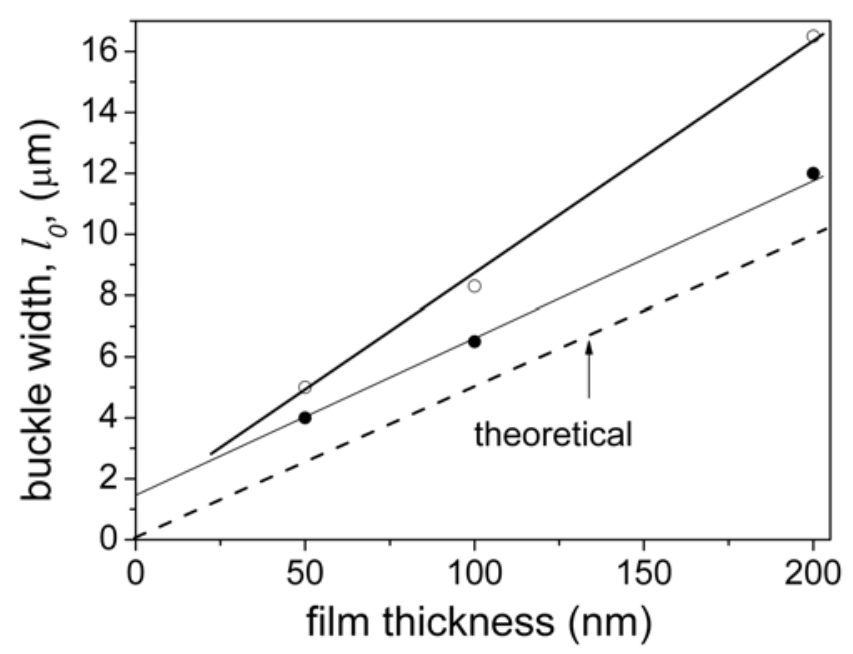

Figure 3.28: Buckle width at critical hydrogen concentration of $\mathrm{Pd} / \mathrm{Nb} / \mathrm{Pd}$ films on $1 \mathrm{~mm} \mathrm{PC}$ (open circles), $0.5 \mathrm{~mm}$ (closed circles) compared to theoretical calculations from Euler's instability (solid lines). The buckle width increases with increase of the film thickness.

From Fig. 3.28 it appears that the buckle width values are slightly larger than the theoretical values. The important point to note is that films on hard substrates need less elastic energy to be stored during hydrogen loading, till delamination occurs. Therefore these films separate from their substrates at lower hydrogen concentrations. The compliance of the substrate also depends on the substrate thickness. The buckle width is larger for the tougher substrates. 


\subsection{Secondary effects}

In this chapter effects were investigated, which appear by applying the controlled buckling technique for films on PC. The influence of electrolyte on the samples results from water absorbing into the polymer and the buoyancy force of electrolyte on the samples. The significance of these effects for the stress measurement by hydrogen loading was investigated. Also the relaxation in the polymer during buckling of the metal films, the interface crack and influence of buckling on the chemical potential was investigated.

\subsubsection{Effect of electrolyte on the stress measurement experiments}

In this work, mainly the electrochemical hydrogen loading was applied. It is important to investigate a possible effect of the electrolyte itself on the measurement and to verify whether this effect significantly influences the adhesion measurement. The buoyancy force of electrolyte on a sample, absorption of water into the polymer and influence of the electrolyte viscosity on the sample bending during stress measurements was expected. Model samples were again $10 \mathrm{nmPd} / 100 \mathrm{nmNb} / 10 \mathrm{nmPd}$ on $1 \mathrm{~mm}$ Polycarbonate.

First the contact of the sample with the electrolyte was investigated. After filling the electrolyte in the loading cell shown in Fig. 3.29, a signal increase of the inductive sensor of about $0.75 \mathrm{~V}$ was observed. This effect is caused by the buoyancy force of the electrolyte (about $3 \mathrm{mN}$ ), which bends the sample. As shown in Fig. 3.29 after that a relaxation of the stress was observed.

a)

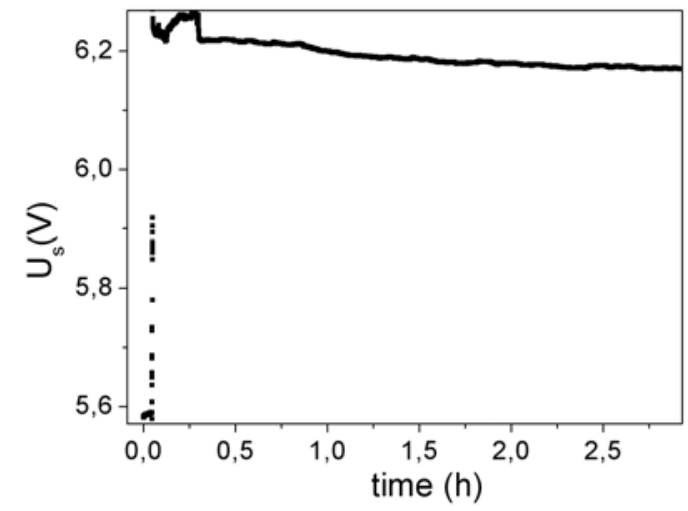

b)

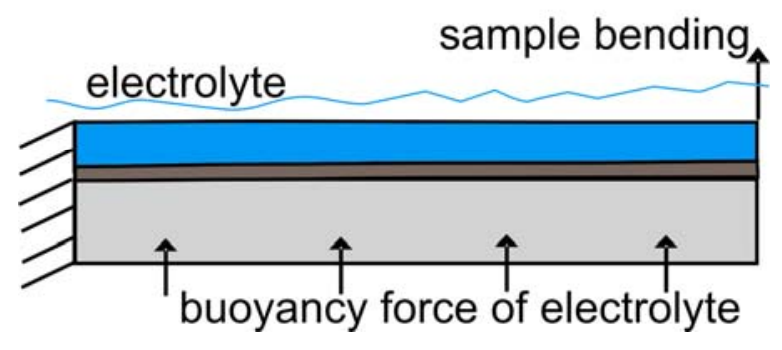

Figure 3.29: a) Bending data of the stress relaxation in the sample, which is under the effect of buoyancy force in electrolyte. The increase of signal from inductive sensor signifies the bending of the sample upstairs due to the buoyancy force of electrolyte. After that the relaxation was observed, b) sample bending in the electrolyte due to the buoyancy force is shown (schematically). 
Relaxation is visible by a decrease of the signal of about $0.1 \mathrm{~V}$ within 3 hours.

Fig. 3.30 shows the signal of the inductive sensor during the first $60 \mathrm{~s}$ after filling the electrolyte into the stress measurement equipment with a mounted sample. The region 1-2 presents the change of the sensor signal by the contact of the inductive sensor with the electrolyte. The possible reasons for this signal change can be the cooling effect of electrolyte on the sensor (a warming effect of the sensor after switching-on was also observed) or/and the change of the electrical field in the vicinity of the sensor due to the polarisation of ions in the electrolyte. The region 2-3 shows the changes of the values due to the lifting of a sample in electrolyte. In the region 3-4 mechanical relaxation of stress caused by buoyancy force is taking place.

a)

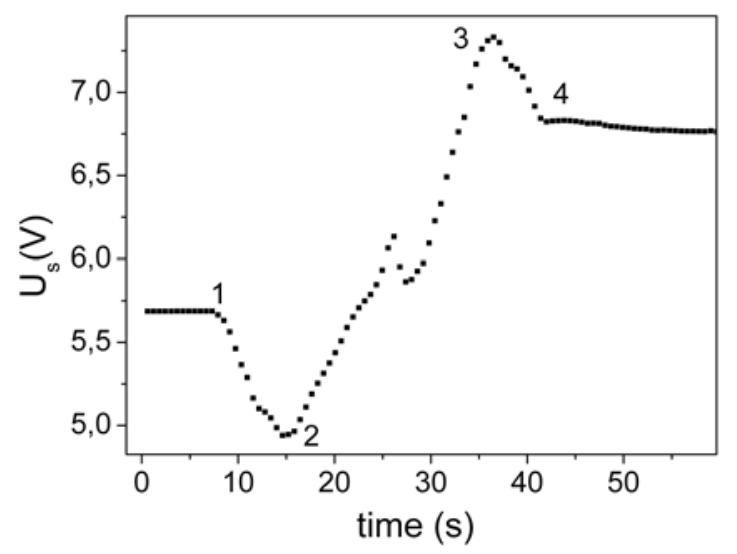

b)

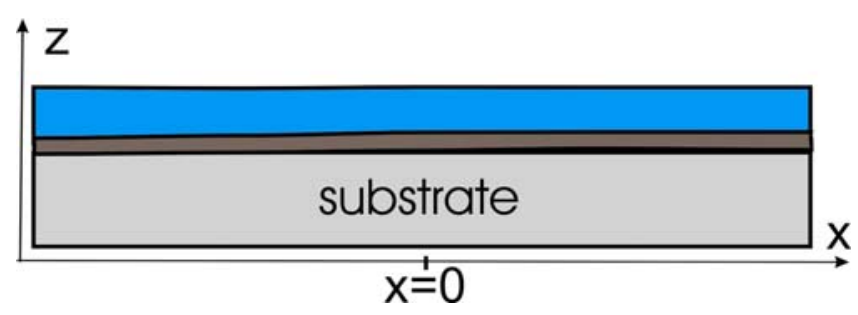

Figure 3.30: a) The insertion of the electrolyte in to the stress measurement equipment, b) the side view of a sample by horizontal geometry of the sample positioning.

The lifting of the sample in the electrolyte can be avoided if the sample is rotated by $90^{\circ}$ around its length axis X (see Fig. 3.30b). In this case there is only an effect of the influence of the electrolyte on the sensor. All measurements described in this chapter were performed with the horizontal geometry of the sample positioning.

Further, the polymer substrate might absorb water. The possible influence of this effect was investigated dipping the sample into pure water. The results are shown in Fig. 3.31. The fast bending up and slow bending down of the sample was observed. 


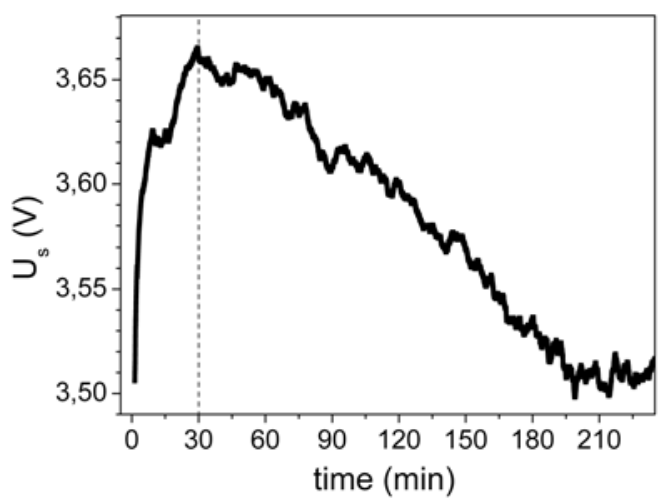

Figure 3.31: Sample bending in the water. The sample bends up for about $30 \mathrm{~min}$, followed by a reverse bending within 3 h. Fast bending occurs within the first 5 min.

Calculating the time, water molecules need to diffuse through a $1 \mathrm{~mm}$ PC plate gives $40.5 \mathrm{~min}$, when the diffusion coefficient of water in PC $D=0.68 \cdot 10^{-6} \mathrm{~cm}^{2} / \mathrm{s}$ [BI89] is used. Thus due to water absorption the free end of the sample bends up by about $10 \mu \mathrm{m}$ within $30 \mathrm{~min}$, as can be seen in Fig. 3.31. This can be explained by water absorption in the bottom side of the sample up to the half width of the substrate. The origin of the following stress relaxation with a signal decrease of about $0.17 \mathrm{~V}$ within 3 hours similar to the process shown in Fig. 3.29 can be as follows: the diffusion of water into the polymer causes softening of the polymer because of a plasticizing effect of the water and/or the scission of water-sensitive bonds such as hydrogen bonds. The stress evolution during the water absorption in the bottom half of the sample and stress relaxation by polymer softening is in the opposite direction and, therefore, both processes counteract. The times for the relaxation process in the electrolyte and the water are similar.

To study the dipping-stress relaxation in the absence of electrolyte the sample was shortly bended down. The subsequent relaxation is plotted in Fig. 3.32. A signal increase of $0.1 \mathrm{~V}$ within 3 hours was observed. 
a)

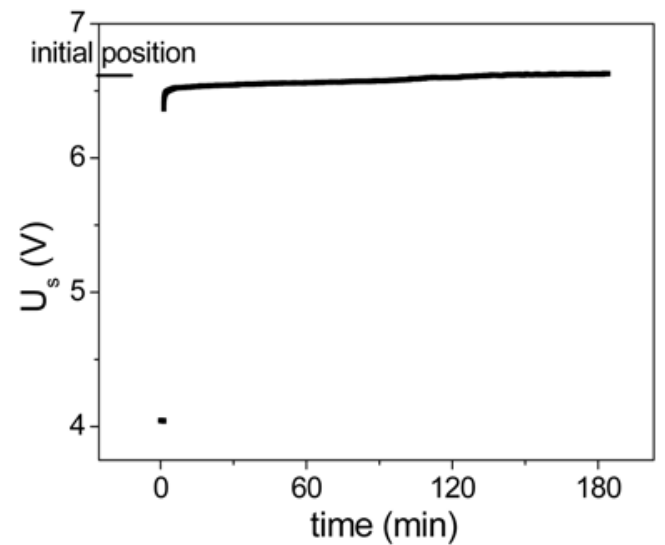

b)

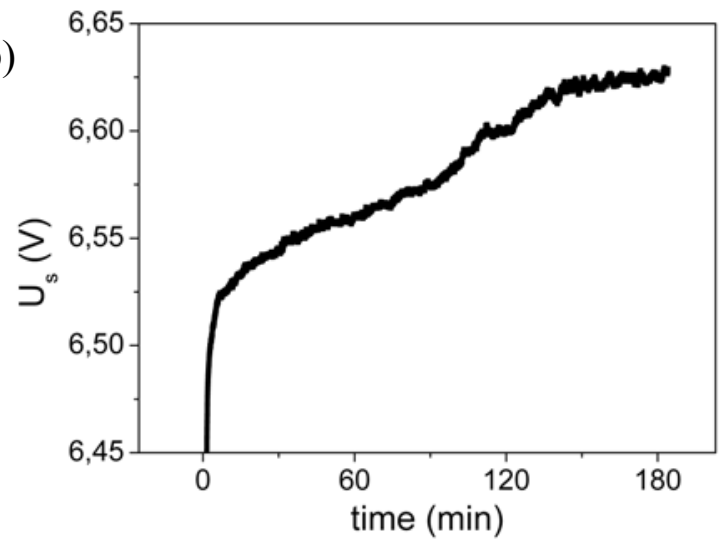

Figure 3.32: Behaviour of the model sample, which was shortly bent down without electrolyte, in order to investigate stress relaxation. The stress relaxation is revealed by the change of the sensor signal of about $0.12 \mathrm{~V}$ during 3 hours. Plot b) shows the same the plot in more detail.

Alternatively the same sample was also shortly bent up, and it showed the same relaxation behaviour. Concluding, the sample relaxation without the electrolyte is of the same order in time as the relaxation in the electrolyte.

The influence of thin protective polymer film which protects the inductive sensor from the electrolyte was also investigated. It was supposed that the protection film might swell in the electrolyte absorbing molecules from the electrolyte and therefore falsify the results of bending measurements. To check that sensor calibration was made without electrolyte, shortly after electrolyte insertion and after some hours in the electrolyte. But this assumption was not confirmed, as it can be seen in Fig. 3.33, where the dependence of the sensor signal on the sensor width is shown with and without electrolyte. The measurements were stable over 24 hours. The slope of the calibration curve was not significantly changed by the presence of electrolyte, meaning no influence of the protection film between the sensor and the sample. The protection film for the inductive sensor has no influence on the measurement. 


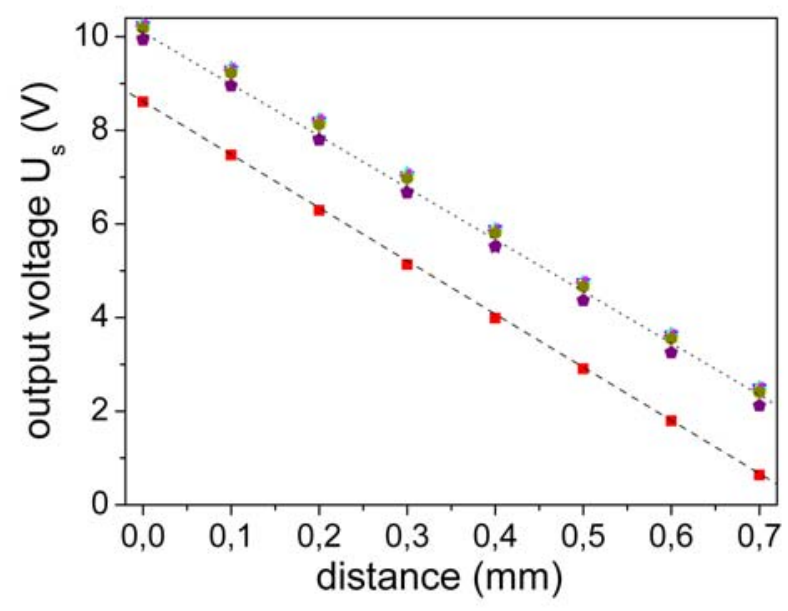

Figure 3.33: Calibration of the inductive sensor for different times during 24 hours. Some datasets were vertically moved for better data illustration. The slope of the calibration curve was not significantly changed during the calibration measurements without electrolyte (red points), after insertion of electrolyte (purple points) and after some hours of electrolyte impact on the protective polymer film (brown points).

As shown in Fig. 3.34, a signal-drift has been observed when the sensor was turned on.

a)

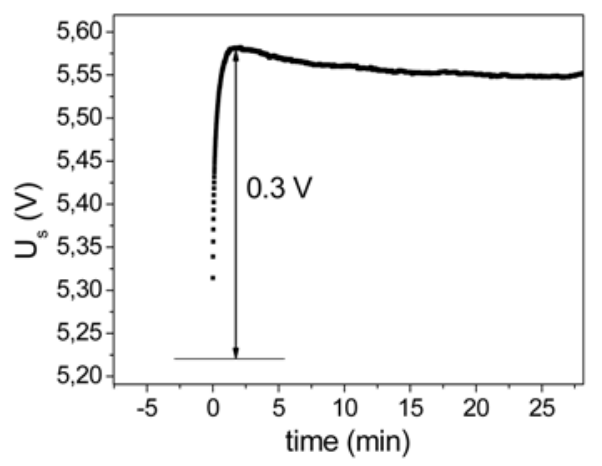

b)

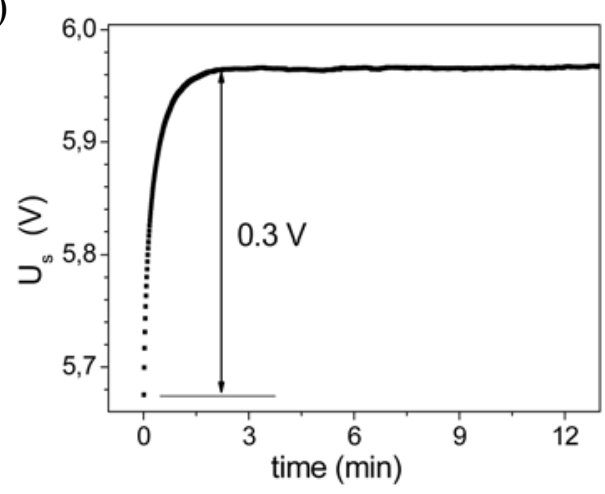

Figure 3.34: Increasing of the measured signal supplied from inductive sensor after turning the inductive sensor on, a) without electrolyte b) in electrolyte.

The voltage change $\Delta \mathrm{U}$ amounts to $0.3 \mathrm{~V}$ within $3 \mathrm{~min}$ in both cases. This effect is ascribed to the warming-up process of the sensor, containing an inductive coil. To avoid this effect the sensor was turned on at least 5 min before the measurement.

Summarizing the electrolyte effects, it is necessary to take the effects of an electrolyte on the measurement results into account and overcome them by calibration. But, the long-time effects such as the stress relaxation in sample lifted in electrolyte, changes of the outside temperature affecting the inductive sensor etc. are not important for short time bending measurements. The water absorption in the bottom side of the sample is also not significant for the bending stress measurements, since the effect is small. 


\subsubsection{Polymer relaxation during buckling process}

Relaxation also occurs during hydrogen loading. As expected the strongest relaxation is found at concentrations above $\mathrm{c}_{\text {crit, }}$, when delamination happens. This can be seen in Fig. 3.35.

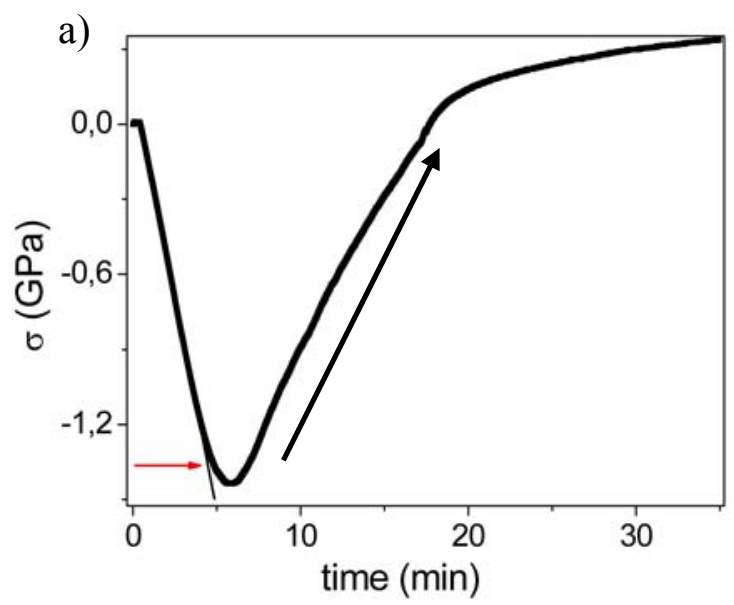

b)

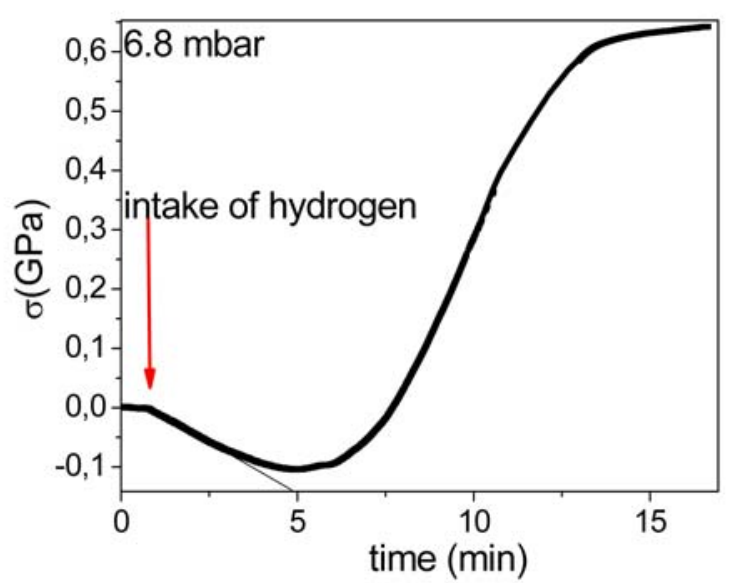

Figure 3.35: Hydrogen loading using electrochemical method (a) and from the gas phase (b) of Pd (10 $\mathrm{nm}) / \mathrm{Nb}(100 \mathrm{~nm}) / \mathrm{Pd}(10 \mathrm{~nm}) / / \mathrm{PC}(1 \mathrm{~mm})$ samples. a) Continuous loading 0-40 \% H/Nb. The arrow marks the start of delamination followed by a bending up of the sample with the stress changing from 1.4 GPa to 0.3 GPa. This bending of the substrate is caused by the relaxation of the polymer. The polymer shows significant changes below the original wrinkles after loading and delamination of the metal layer (see Fig. 3.36). b) Stress curve during hydrogen gas loading at 6.8 mbar.

The polymer relaxation under the area of a buckle was studied directly after setting in of delamination (marked with the red arrow in Fig. 3.35a), where the stress in the film-polymersystem decreases and even turns back above the initial stress. To avoid confusion, this does not mean that tensile stress appears in the film, as the zero point of the compressive stress is arbitrary. Nevertheless it can be expected that relaxation not only occurs in the film itself, but also in the substrate, because the exceeding of the relaxation stress over the zero point with about $50 \%$ detached area is larger than the intrinsic stress of $-280 \mathrm{MPa}$ in the film, which was measured using the $\sin ^{2} \psi$ method by determining the shift of the diffraction peak during the rotation of the sample by an angle $\Psi$. The relaxation of the compressive stress during gas hydrogen loading, which can be seen in Fig 3.35b, is also linked to the buckling of the film.

The film-polymer-system bends during the polymer relaxation. This effect takes place relatively slowly. One idea to test if the PC-relaxation mainly occurs below the buckles is to look at the PC substrate after removing the buckled film. A light microscopy image of the PC substrate after removing the metal film shows significant optical changes of PC in the field of original wrinkles, 
as presented in Fig. 3.36. There dark lines appear in the places of original wrinkles. By SIMSmeasurements it has been shown that there does not remain any $\mathrm{Nb}$ after removing the film, neither on the black lines, nor in the regions in-between, see Fig 3.37. Therefore, the black lines indicate changes in the PC-substrate itself. An interpretation of these observations is given below.

a)

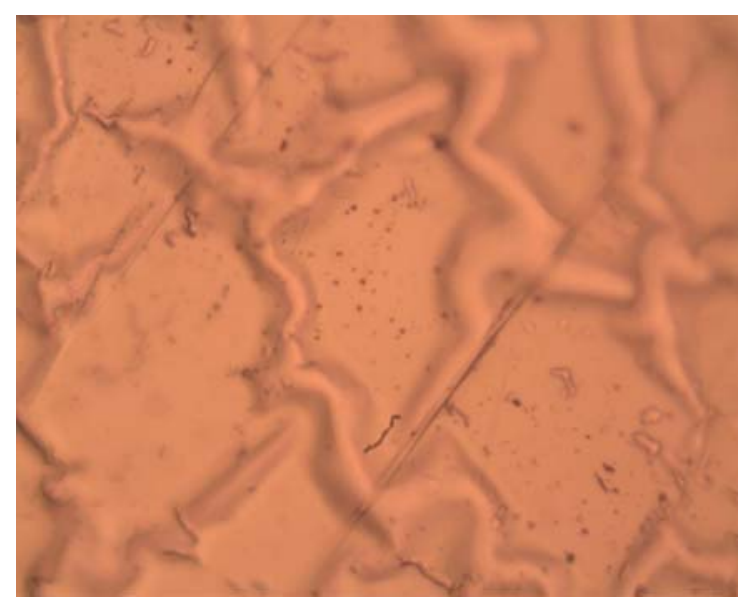

b)

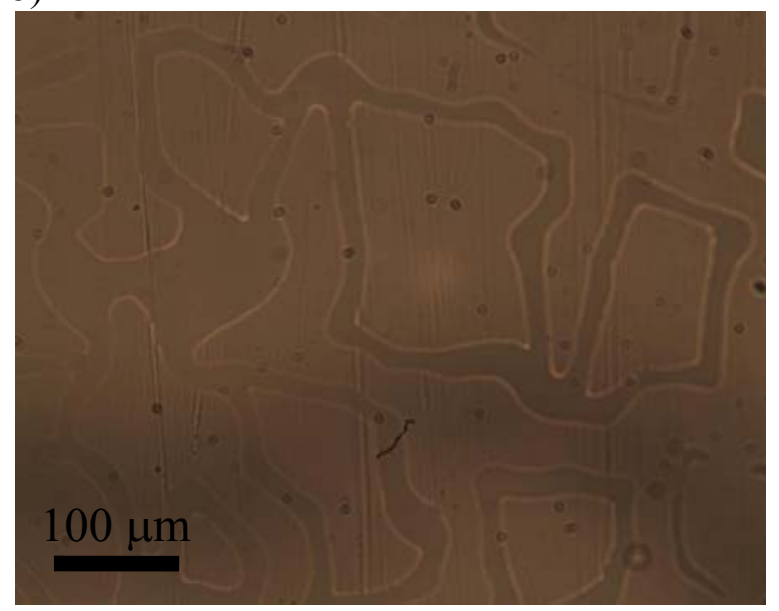

Figure 3.36: A light microscopy image of the PC substrate after removing the metal film shows significant optical changes of PC in the field of original wrinkles a) light microscopically image of a buckled film (top view), b) the PC-surface after removing of the buckled metal film. The dark lines are the regions of buckles. (The two images do not represent the same sample area)

As can be seen in Fig. 3.35, the film-polymer-system relaxes directly after the first wrinkles were formed. Therefore this relaxation effect is directly linked to wrinkles and it can be suggested that the relaxation of the areas in the polymer under the formed buckles occurs. SIMS analysis was performed in order to provide hints of structural changes in the polymer. The metal film inside of the crater shown in Fig. 3.37 was removed by the Ar and Ga ion beam and the analysis continued into the polymer substrate as shown in the SIMS depth profile of Fig. 3.37. 

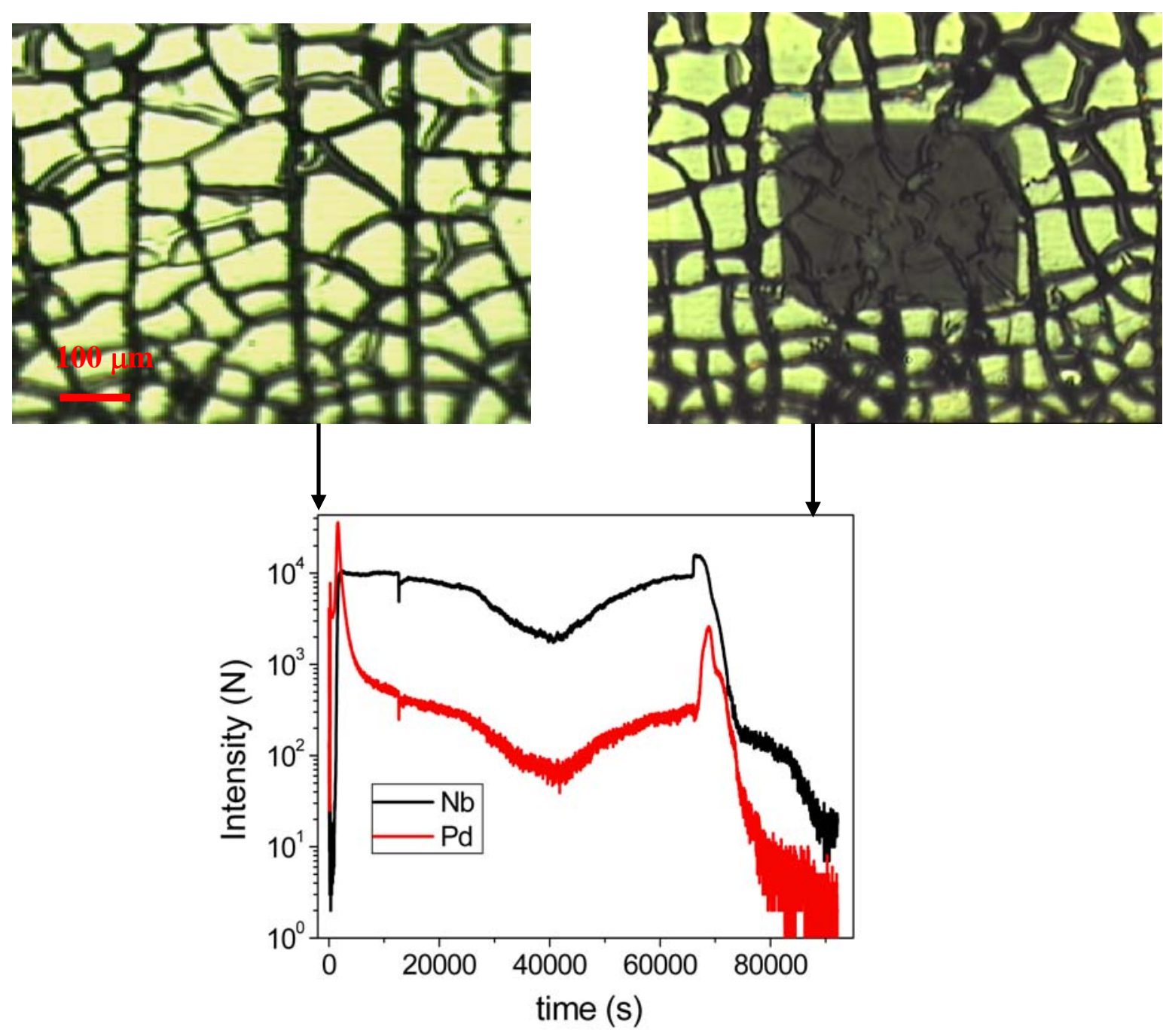

Figure 3.37: Depth profile (SIMS) and light microscopy image of the layer before and after the SIMSmeasurement. In the beginning the metal film layers $\mathbf{P d}, \mathbf{N b}$ and Pd are clearly visible in the depth profile, but from about $9 * 10^{4} \mathrm{~s}$ on they vanish and are not detectible any longer. Therefore, after the depth profiling the surface in the analysed region of analysis (dark square) only consists of polymer; while the wrinkles structures are still remaining. This observation gives evidence for the assumption that the appearance of the dark lines relates to changes in the PC substrate.

The original wrinkle structures are directly visible in the polymer. This effect might be interpreted as an indication for a local modification of the polymer. This modification can be within the polymers, but also on its surface. The modifications in the polymer were studied by polarization microscopy (proof of the rotation of the polarization level of the white light in the polymer by mechanically differently deformed areas). The resulting colored images exhibit a weak dependency of polymer areas under the buckles compared to polymer surface outside the buckles on the polarization angle. This result provides further evidence for the interpretation that the surface topography of the polymer is changed by relaxation. 
The magnitude of the polymer relaxation depends on the width of the buckled area and, therefore, on the film thickness (see Fig 3.7). $\mathrm{Pd}(10 \mathrm{~nm}) / \mathrm{Nb} / \mathrm{Mo}(10 \mathrm{~nm})$ films on $1 \mathrm{~mm} \mathrm{PC}$ with $50 \mathrm{~nm}, 100 \mathrm{~nm}$ and $200 \mathrm{~nm}$ Nb-layers were stepwise electrochemically loaded. The Hconcentration ranged from 0 to $30 \% \mathrm{H} / \mathrm{Nb}$, with loading steps of $1 \% \mathrm{H} / \mathrm{Nb}$. In Fig. 3.38 the bending data of these samples are shown.

a)

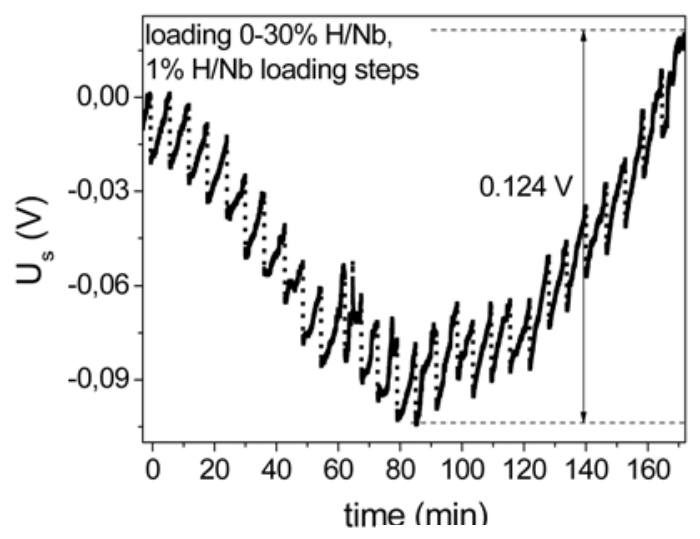

b)

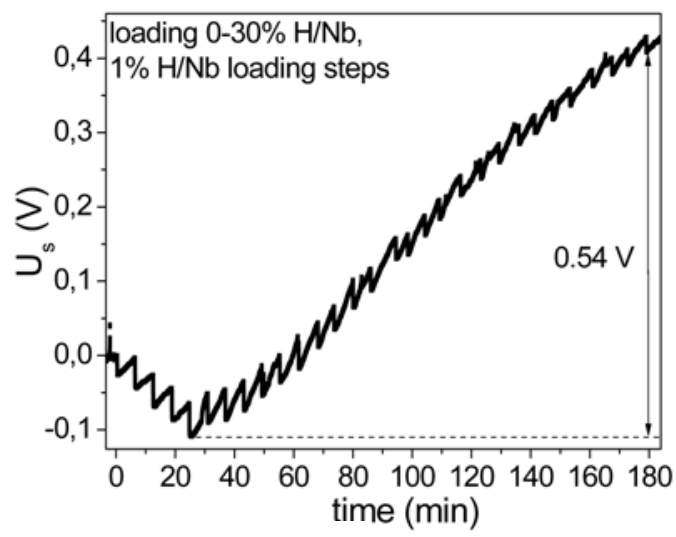

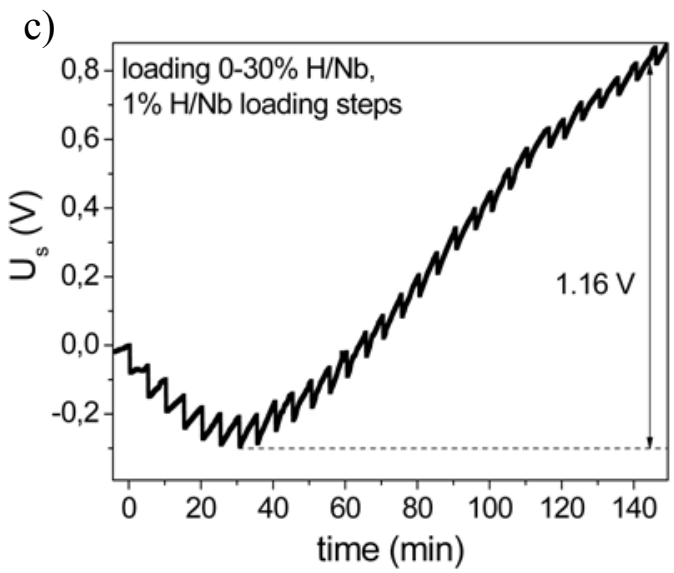

Figure 3.38: Bending data of stepwise electrochemically hydrogen loaded Pd (10nm)/Nb/Mo $(10 \mathrm{~nm}) / / \mathrm{PC}(1 \mathrm{~mm})$ samples with Nb-layer a) $50 \mathrm{~nm}$, b) $100 \mathrm{~nm}$, c) $200 \mathrm{~nm}$. The general curve progression shows a larger relaxation effect for the larger film thickness.

The magnitude of the overall relaxation as shown in Fig. 3.38 increases with increasing film thickness, which affects the intrinsic stress of the films and the width of the buckles. The most important quantity in the relaxation process is the buckle width, which determines the total delaminated area and, therefore, the area where polymer relaxation takes place. The delaminated area at $30 \% \mathrm{H} / \mathrm{Nb}$ is largest for the $200 \mathrm{~nm} \mathrm{Nb}$ layer, because of the smaller critical concentration and a larger buckle width. Therefore the relaxation effect of the $200 \mathrm{~nm}$ film is bigger. 


\subsubsection{Buckle formation observed in the stress curve}

Jumps in the stress curve were detected during stepwise hydrogen loading, which indicated that the formation of single buckles was recorded, see Fig. 3.39. The stress leaps of about 2-3 MPa occurred within $1 \mathrm{sec}$. This process differs from the relaxation phenomena after each loading step shown in Fig. 3.38. A similar picture has been seen when the buckle formation was observed by light microscopy. It was observed that the buckles appeared one after the other, while they occurred at positions amounting about a half length of the already existing wrinkles, as it was shown in Chap. 3.1.5. To verify this interpretation, a special experiment was performed, during which only the area visible in the light microscope (a square of $2 \times 2 \mathrm{~mm}^{2}$ ) was H-loaded, while the rest of the sample was covered with nail polish and was not loaded). Hydrogen loading of the covered film was not considered. The idea was to link the voltage leaps with a single wrinkle formation. However, it was found that the inductive sensor is not sensitive enough to detect the sample bending resulting from the charging of a very small area. Therefore there is a lack of a clear proof of that phenomenon to this day. Nevertheless from the time correlation, as described above, it can be assumed that the origin of the voltage leaps lies in the buckle formation.

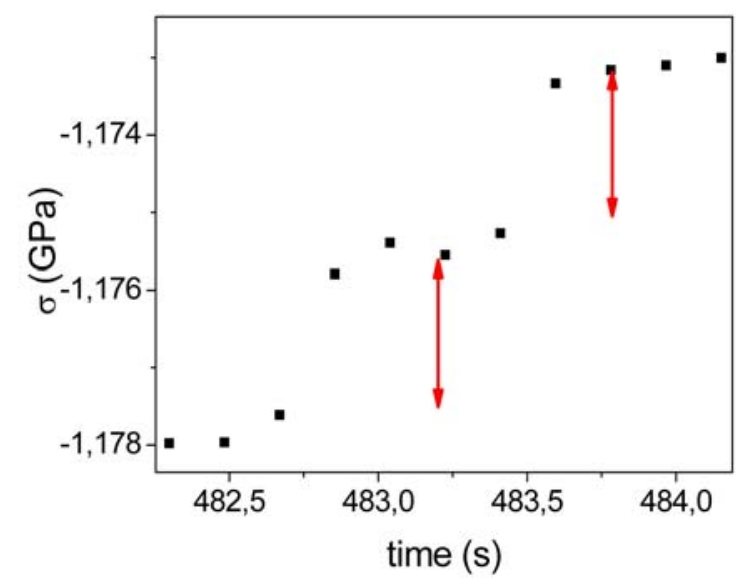

Figure 3.39: Single leaps in the measured mechanical stress, which correlate with buckle formation during electrochemical hydrogen loading. The leap height is about 2-3 MPa, which seems to be a stress value released during the formation of a new wrinkle. 


\subsubsection{Investigation of crack interfaces}

Investigating the metal detachment from the polymer substrate the critical question concerns the cracking interface. The model of adhesion energy measurement by hydrogen loading assumes that the metal-polymer-system breaks right at the interface between the metal and the polymer. If the crack would appear inside the polymer or the metal-layer, it would be impossible to evaluate the interfacial energy.

If the crack propagates in the metal layer Secondary Ion Mass Spectroscopy (SIMS) detect the palladium or niobium ions on the surface after delamination. A highly loaded sample was peeled off from the substrate using an adhesive tape. It turned out that there were no metal ions detected at the polymer afterwards. Thus, cracking in the metal film can be excluded.

If the crack propagates in the polymer, polymer should be left on the backside of the metal film. SIMS analysis of the back side of the detached metal film is not conclusive, as the differentiation between polymer residues and sorbed residual hydrocarbons in the UHV is not possible.

But, it can be argued that, if the crack appeared inside the polymer, the metal-polymer interface itself would be unimportant and the adhesion values would be independent of the metal. It has been shown that this is not the case (see Chap. 3.4). Therefore it is concluded that the metalpolymer system breaks at the interface. 


\subsection{Influence of film delamination on the chemical potential of hydrogen}

An interesting phenomenon detected during hydrogen loading of $\mathrm{Pd} / \mathrm{Nb} / \mathrm{Pd}$ samples on $1 \mathrm{~mm}$ PC regards the decrease of the chemical potential of hydrogen (emf), when the sample detaches. To investigate this effect, the films were stepwise loaded with steps of $1 \% \mathrm{H} / \mathrm{Nb}$ and waiting times of $1 \mathrm{~min}$ between the loading steps. The measured voltage of the inductive sensor during $\mathrm{H}$ loading of a film with $50 \mathrm{~nm} \mathrm{Nb}$ thickness is shown in Fig. 3.40. As it appears from about $27 \%$ $\mathrm{H} / \mathrm{Nb}$ the slope of the voltage-curve increases, revealing film buckling.
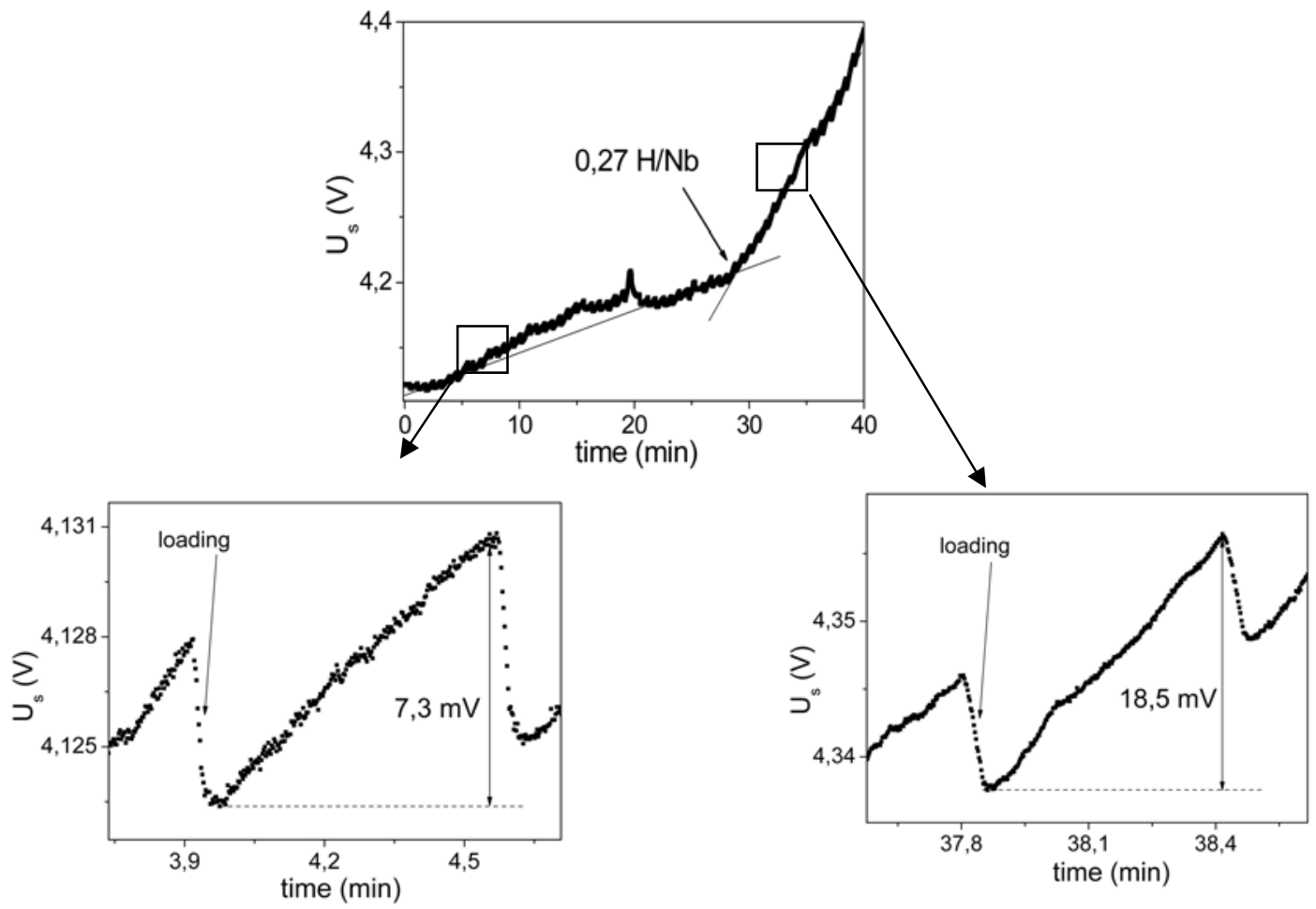

Figure 3.40: H-loading of $\mathrm{Pd} / \mathrm{Nb} / \mathrm{Pd} / / \mathrm{PC}$ sample with $50 \mathrm{~nm}$ Nb-layer. The plots show the inductive sensor signal and, therefore, the bending behavior of the sample during hydrogen loading. Larger relaxation after each loading step was observed at hydrogen concentrations higher than $0.27 \mathrm{H} / \mathrm{Nb}$. a) bending curve of the whole hydrogen loading sequence $0-40 \% \mathrm{H} / \mathrm{Nb}, \mathrm{b}$ ) magnification of a loading step at the beginning of hydrogen loading, c) magnification of a loading step after onset of buckling.

The accompanying emf curve of the sample in the concentration range around $27 \% \mathrm{H} / \mathrm{Nb}$ is shown in Fig. 3.41. 


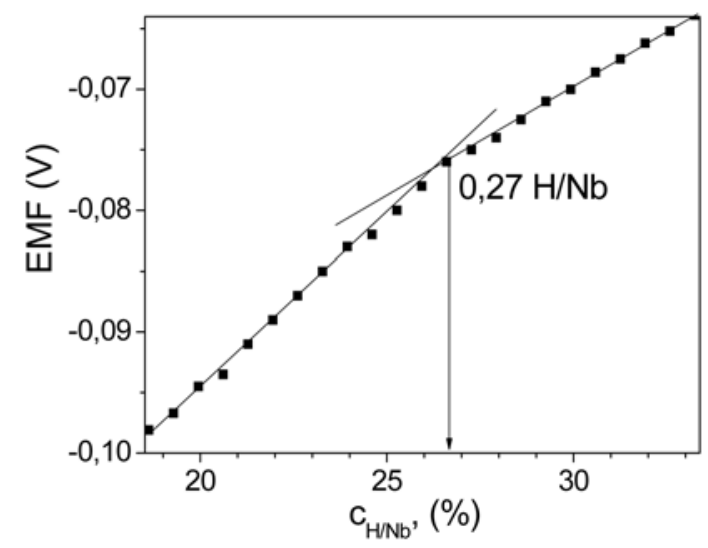

Figure 3.41: $\mathrm{Emf}$ of $\mathrm{Pd} / \mathrm{Nb} / \mathrm{Pd} / \mathrm{PC}$ sample with $50 \mathrm{~nm} \mathrm{Nb}$-layer around $0.27 \mathrm{H} / \mathrm{Nb}$, where film detachment sets in. The emf values were recorded about 1 min after each $\mathrm{H}$-loading step, when the system was assumed to be in thermodynamic equilibrium. As it appears, at the onset of buckling the slope of the emf-curve slightly decreases.

It can be seen that the slope of the emf-hydrogen concentration curve, as shown in Fig. 3.41, at $27 \% \mathrm{H} / \mathrm{Nb}$ slightly decreases, and this effect is clearly correlated with the onset of buckling.

This effect has been observed for $\mathrm{Pd} / \mathrm{Nb} / \mathrm{Pd}$ films with $100 \mathrm{~nm}$ and $200 \mathrm{~nm}$ of Nb-layers as well, and the magnitude of the effect was even larger, see Fig. 3.42.
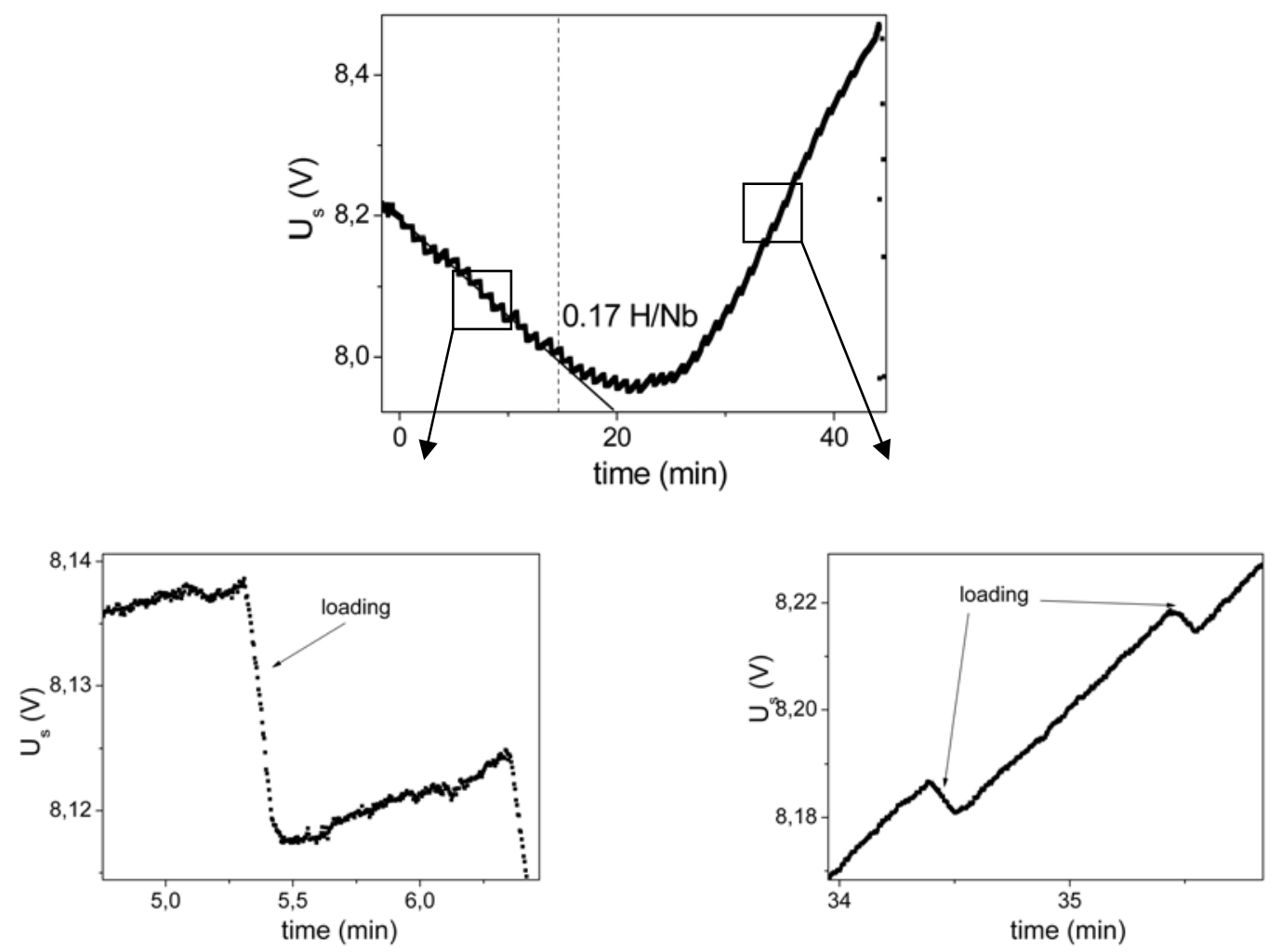

Figure 3.42: Bending behavior of H-loading of $\mathrm{Pd} / \mathrm{Nb} / \mathrm{Pd} / \mathrm{PC}$ sample with $100 \mathrm{~nm} \mathrm{Nb}$-layer. a) bending curve of the whole hydrogen loading sequence $0-40 \% \mathrm{H} / \mathrm{Nb}, \mathrm{b}$ ) magnification of a loading step at the beginning of hydrogen loading, c) magnification of a loading step after onset of buckling. 
The relaxation is stronger in the region of the bending curve in Fig. 3.42 where the buckling rate increases. The change in the emf-slope at the onset of buckling at $17 \% \mathrm{H} / \mathrm{Nb}$ for a $100 \mathrm{~nm} \mathrm{Nb}$ film is shown in Fig. 3.43.

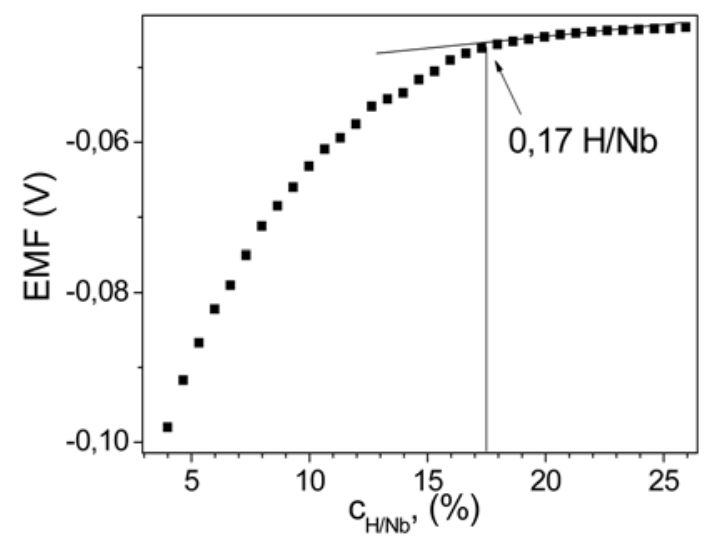

Figure 3.43: Emf of $\mathrm{Pd} / \mathrm{Nb} / \mathrm{Pd} / / \mathrm{PC}$ sample with 100nm Nb-layer nearby of $0.17 \mathrm{H} / \mathrm{Nb}$. The slope of emf-curve changes at this concentration.

The $200 \mathrm{~nm}$ film shows the large relaxation effect of the metal film (and also the polymer relaxation, see Chap. 3.2.2), appearing at $13 \% \mathrm{H} / \mathrm{Nb}$, see Fig. 3.44.
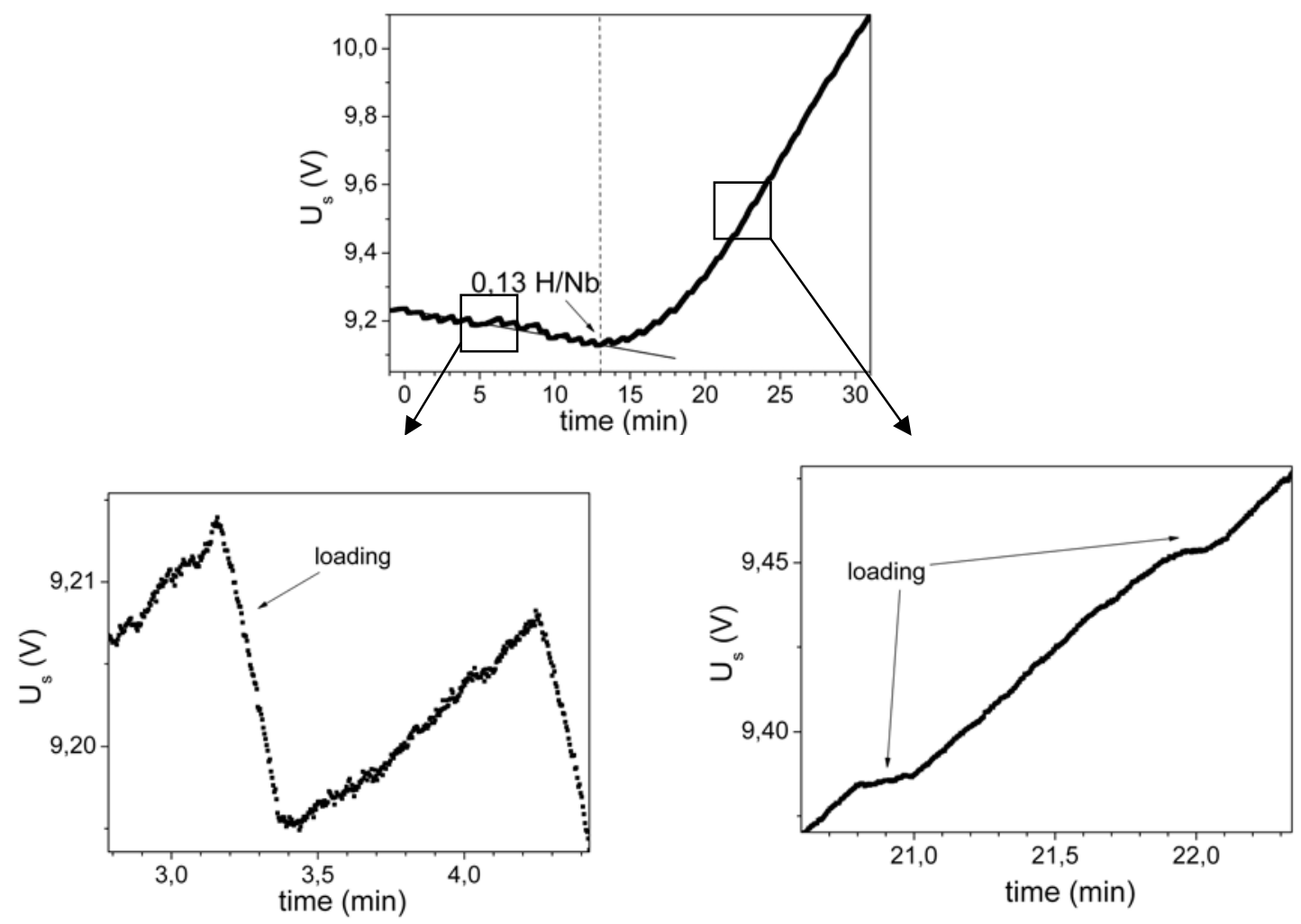

Figure 3.44: stepwise H-loading of Pd/Nb/Pd/PC sample with 200nm Nb-layer. 
The related emf values even have a maximum at $13 \% \mathrm{H} / \mathrm{Nb}$ and decreases afterwards, see Fig. 3.45. This decrease correlates with a film buckling which starts also at hydrogen concentration of $13 \% \mathrm{H} / \mathrm{Nb}$

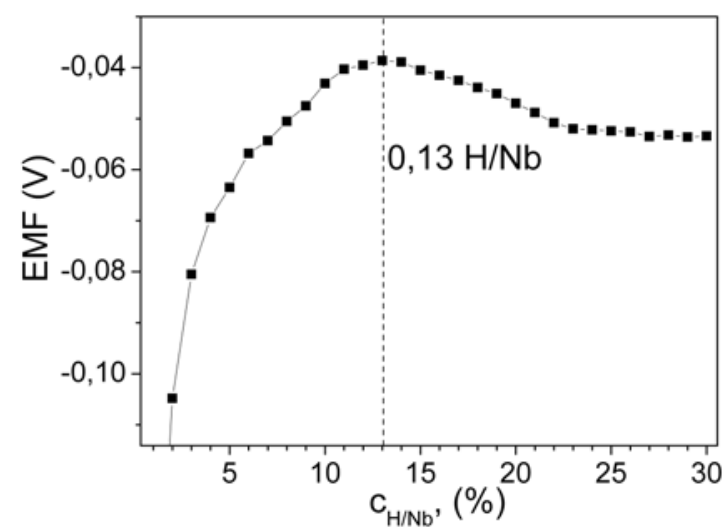

Figure 3.45: Emf of $\mathrm{Pd} / \mathrm{Nb} / \mathrm{Pd} / \mathrm{PC}$ sample with 200nm Nb-layer nearby of $0.13 \mathrm{H} / \mathrm{Nb}$. The decrease of emf values after $13 \% \mathrm{H} / \mathrm{Nb}$ which is correlated with the beginning of buckling was detected.

In Tab. 3.2 the measured slopes of the bending curves for the discussed samples are summarized. All films were loaded with $1 \% \mathrm{H} / \mathrm{Nb}$ per minute.

Table 3.2: The slopes which characterize the general bending curves progression by hydrogen loading of Pd/Nb/Pd films with $\mathrm{Nb}$ thickness of $50 \mathrm{~nm}, 100 \mathrm{~nm}$ and $200 \mathrm{~nm}$ during hydrogen loading are shown. The slope during buckling process is larger for films with larger thickness.

\begin{tabular}{|l|l|l|l|}
\hline \multirow{2}{*}{ Film } & \multicolumn{3}{|l|}{ Slope of the bending curve $\left(* 10^{-3} \mathrm{~V} / \mathrm{min}\right)$} \\
\cline { 2 - 4 } & before buckling & $\begin{array}{r}\text { after beginning of } \\
\text { buckling }\end{array}$ & $\begin{array}{l}\text { after beginning of } \\
\text { buckling (relative to the } \\
\text { slope before buckling })\end{array}$ \\
\hline $\mathrm{Pd} / \mathrm{Nb} \_50 \mathrm{~nm} / \mathrm{Pd}$ & 2.2 & 11 & 8.8 \\
\hline $\mathrm{Pd} / \mathrm{Nb} \_100 \mathrm{~nm} / \mathrm{Pd}$ & -13.5 & 28.7 & 42.2 \\
\hline $\mathrm{Pd} / \mathrm{Nb} 200 \mathrm{~nm} / \mathrm{Pd}$ & -8 & 70 & 78 \\
\hline
\end{tabular}

The slope change in the general progression of the bending curves from the direction which signifies compressive stress increase to the direction of stress relaxation is linked with the onset of buckling. Tab. 3.2 summarizes the slopes of the bending curves before and after onset of buckling. For thicker films the relaxation process is more intensive, since the buckles have larger size and at the same hydrogen concentration the buckled surface of the sample is larger. The origination of this effect was discussed in Chap. 3.2.

Accompanied to the slope of the bending curves also the slope of the related emf-curves changes as can be seen in Tab. 3.3. 
Table 3.3: Comparison of the slopes of emf curves for films with different film thickness. The decrease of emf by buckling process occurs faster for the films with larger thickness.

\begin{tabular}{|l|c|c|c|}
\hline Nb thickness & 50 & 100 & 200 \\
\hline emf slope before buckling, & 2.8 & 2.01 & 3.3 \\
$\mathrm{~s}_{1}, \frac{V}{C_{H / N b}(\%)}\left(\cdot 10^{-3}\right)$ & 1.81 & 0.32 & -1.45 \\
\hline emf slope after onset of & & & \\
buckling, $\mathrm{s}_{2}, \frac{V}{C_{H / N b}(\%)}\left(\cdot 10^{-3}\right)$ & -0.99 & -1.69 & -4.75 \\
\hline $\begin{array}{l}\text { Relative change of slope, } \\
\mathrm{s}_{1}-\mathrm{s}_{2},\left(\cdot 10^{-3}\right)\end{array}$
\end{tabular}

The larger emf decrease for films with more stress release by hydrogen loading of $\mathrm{V}-\mathrm{Fe} 8$ at $\%$ films prepared at different temperature was found by Gemma et al. [G07]. A larger stress release was found for the films with smaller domains. The mechanism of the stress release was assumed to be grain boundary sliding. The mechanism of the stress release for the films in this work is more obvious. It is the buckling process of the metal films under compressive stress during hydrogen loading.

To summarize, the consideration of the buckling process is very important during the investigation of the chemical potential. The stress release caused by the buckling process influences directly the chemical potential of hydrogen, which is decreased. The decrease of the chemical potential increases the solubility of hydrogen in the metal film (see also [B65]). So, a buckled film can absorb more hydrogen atoms compared to film bonded on substrate. 


\subsection{Adhesion energy calculations, results for different interface layers. Alternative methods for adhesion energy calculation: Gille and Rau formula and peel test}

Additionally to the $\mathrm{Pd} / \mathrm{Nb} / \mathrm{Pd}-\mathrm{PC}$ system presented in the previous chapters measurements on system with different metal layers have been performed. For these systems the resulting critical hydrogen concentrations and critical stresses for buckling, determined by means of the controlled buckling technique, will be summarized in the following. From the critical values the respective adhesion energies have been calculated. The results of these calculations will be presented for all different systems analysed in the present work. For comparison, also results on the adhesion energies investigated applying the theory of Gille and Rau will be given. Furthermore, the influence of substrates pre-treatment on the adhesion energies will be analysed.

\subsubsection{Adhesion energy calculation for $\mathrm{Pd} / \mathrm{Nb} / \mathrm{Pd}$ films on $\mathrm{PC}$}

The adhesion energy of metal films on rigid substrates can be calculated modelling the physical constraints of the controlled buckling method, described in Chap. 1.2. Thereby the adhesion energy can be calculated from the slope of the critical values of H-concentration or stress plotted over the square root of the inverse film thickness. Thin $\mathrm{Pd} / \mathrm{Nb} / \mathrm{Pd}$ film packages with $\mathrm{Nb}$ thicknesses of 50, 100, $200 \mathrm{~nm}$ (see Chap. 2.1) deposited onto polycarbonate (PC) substrates of 1 mm thickness (Goodfellow) were hydrogen loaded. PC-substrates of this thickness were found to act like rigid substrates, see Chap.3.1.3. The PC-surface was pre-treated by short dipping it into isopropanol. From XRD-measurements it is known that both Pd and Nb-layers had grain sizes in the 10-100 nm range, which is in fact far below the size of the buckles. In this work delamination occurs at the interface between Pd and PC, see Chap. 3.2.4. Because of the large thickness of the $\mathrm{Nb}$-layer, elastic properties of the metal film package are dominated by the $\mathrm{Nb}$-layer, especially for the $200 \mathrm{~nm}$ layer. For simplification, the contribution of the thin Pd-layers has been neglected here. Controlled hydrogen loading was performed applying loading steps of $\Delta \mathrm{c}_{\mathrm{H}}=0.01 \mathrm{H} / \mathrm{Nb}$. Thereby the onset of film buckling upon hydrogen loading appeared to be rather sharp (Chap.3.1.1): it occurs within $0.01 \mathrm{H} / \mathrm{Nb}$. Therefore, the error for the determined critical hydrogen concentration is estimated to be in this range.

In Chap. 3.1.1 it was shown that the stress evolving due to hydrogen loading deviates from a linear development, when buckling sets in. It was shown that this effect results from stress 
relaxation. The critical values at $2 \%$ and $10 \%$ deviation as shown in Fig 3.46 were applied for comparative adhesion energy calculations.

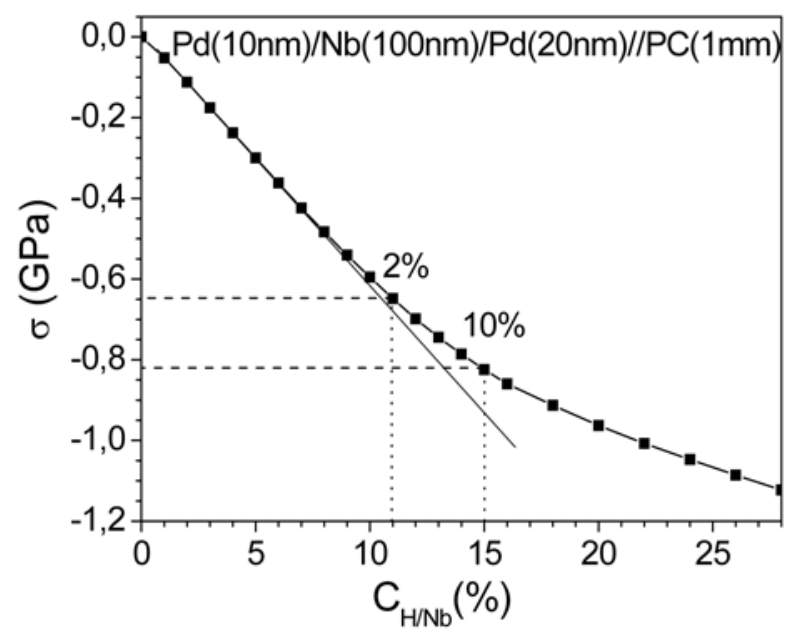

Figure 3.46: Stress curve, measured by stepwise electrochemical hydrogen loading of $\mathrm{Pd} / \mathrm{Nb} / \mathrm{Pd}$ film on $1 \mathrm{~mm}$ PC. Due to relaxation effects, the stress-curve deviates from the initial linear development, when buckling sets in. Deviations of $2 \%$ and $10 \%$ respectively are marked with the dashed lines.

The determination of the critical stress can be performed in two ways: By taking the stress value at $2 \%$ deviation from the extrapolated linear development which corresponds to the onset of delamination by optical observing of the surface in most experiments (see Fig. 3.3). For comparison critical stress were also defined for $10 \%$ deviation and used to calculate adhesion energies.

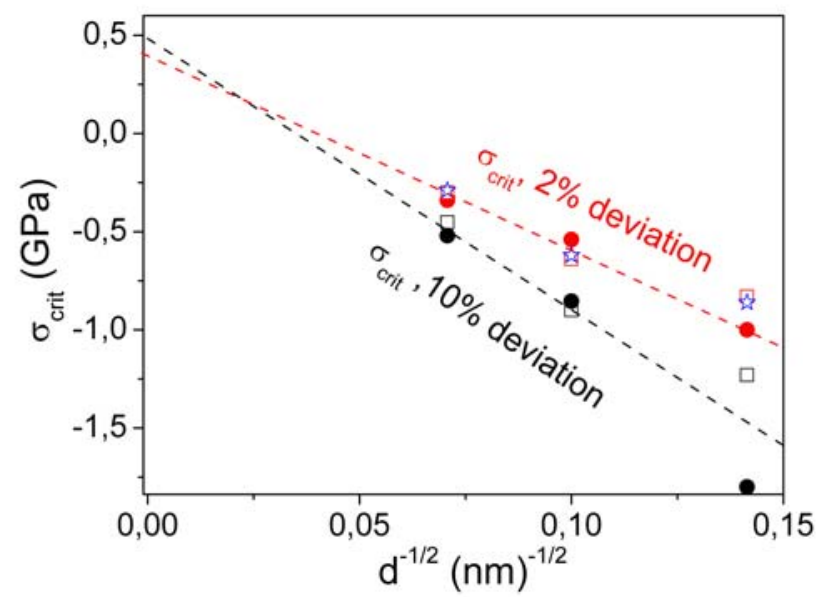

Figure 3.47: Critical stress for delamination for $\mathrm{Pd}(10 \mathrm{~nm}) / \mathrm{Nb}(50,100,200 \mathrm{~nm}) / \mathrm{Pd}(10 \mathrm{~nm})$ on 0.5 mm (closed circles) and $1 \mathrm{~mm}$ (opened squares PC substrate obtained from stress measurements and plotted versus inverse square root of film thickness $d$. The red dots are taken for $2 \%$ deviation of the stress curve from linear behaviour, the black ones for 10\%. The starlets are obtained from measurements of the critical hydrogen concentration $\mathrm{H} / \mathrm{Nb}$ by determining the delaminated area. 
The adhesion energies obtained from the data shown in Fig. 3.47 are given in Tab. 3.4. Thereby the adhesion energies were calculated from the slopes of the dash lines in Fig 3.47 applying Eq. (1.33).

Table 3.4: Adhesion energy values calculated for Pd on PC using critical stress values for buckling.

\begin{tabular}{|l|c|c|}
\hline & \multicolumn{2}{|c|}{$\begin{array}{c}\text { Referring to critical stress } \\
\text { applying Eq. (1.33) }\end{array}$} \\
\hline Deviation of the critical value & $2 \%$ & $10 \%$ \\
\hline Adhesion energy $\gamma,\left(\mathrm{J} / \mathrm{m}^{2}\right)$ & $0.32 \pm 0.08$ & $0.8 \pm 0.2$ \\
\hline
\end{tabular}

Adhesion energy calculation can also be performed from the critical hydrogen concentrations using equation (1.34). These concentrations obtained by hydrogen loading of films on $1 \mathrm{~mm}$ PC are given in Fig. 3.48 as a function of the square root of the inverse film thickness. Thereby the critical hydrogen concentrations were defined as those $\mathrm{c}_{\mathrm{H} / \mathrm{Nb}}$ values where buckling was observed optically. It was shown in Chap. 3.1.1 that these values correspond to the stress values deviating by $2 \%$ from the linear stress development. The obtained critical concentrations vary within $\pm 1 \%$ $\mathrm{H} / \mathrm{Nb}$. Applying equation (1.34) from the slope of the straight line, an adhesion energy $\gamma_{\mathrm{Pd} / \mathrm{PC}}=0.4( \pm 0.1) \mathrm{J} / \mathrm{m}^{2}$ is derived.

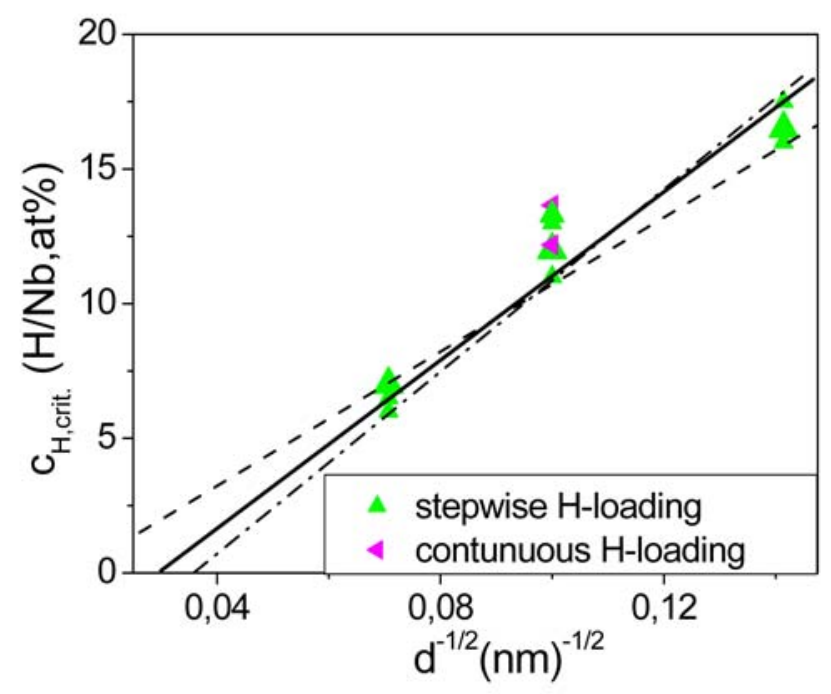

Figure 3.48: Measured critical $\mathrm{H}$-concentrations as a function of the square root of the inverse $\mathrm{Nb}$ film thickness. Sets of data points were obtained for different films on $1 \mathrm{~mm}$ PC that were independently prepared using the same preparation conditions. The obtained critical concentrations vary within \pm 1 at\% $\mathrm{H} / \mathrm{Nb}$.

In Fig. 3.49 the dependency of the critical hydrogen concentrations on the Nb-film thickness of the $\mathrm{Pd} / \mathrm{Nb} / \mathrm{Pd}$ layers on $1 \mathrm{~mm} \mathrm{PC}$ is compared for the two different degrees of deviation. For the assigned stress curves see Fig. 3.5. The adhesion energy values have been calculated from the slope of the resulting curves applying Eq. (1.34). 


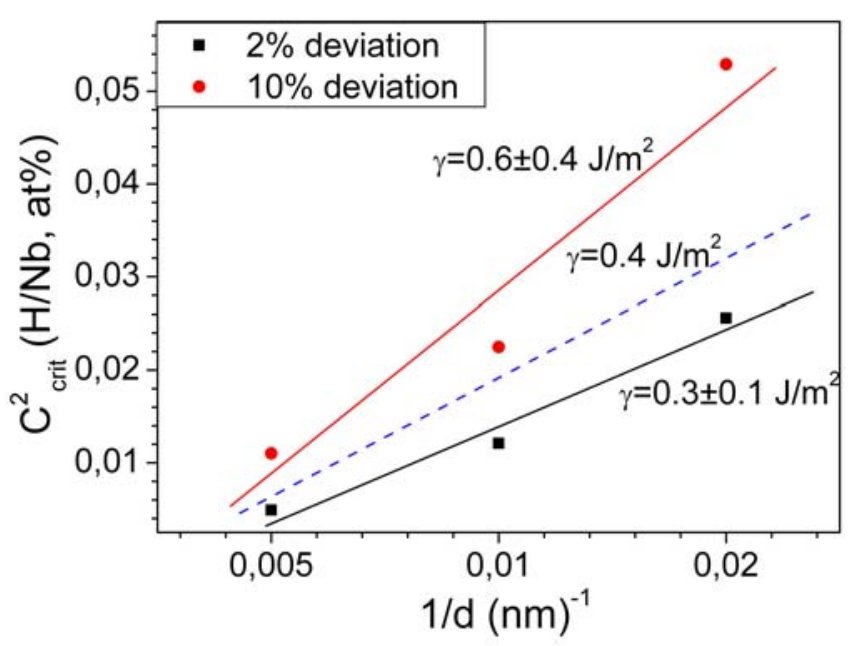

Figure 3.49: $c^{2}{ }_{\text {crit }}$ versus inverse film thickness for $\mathrm{Pd} / \mathrm{Nb} / \mathrm{Pd}$ on $1 \mathrm{~mm}$ PC. For comparison the line for $\gamma=1 \mathrm{~J} / \mathrm{m}^{2}$ is also plotted. The resulting adhesion energy values have been calculated applying Eq. (1.34).

The same plot for $\mathrm{Pd} / \mathrm{Nb} / \mathrm{Pd}$ films on $0.5 \mathrm{~mm} \mathrm{PC}$ is shown in Fig. 3.50.

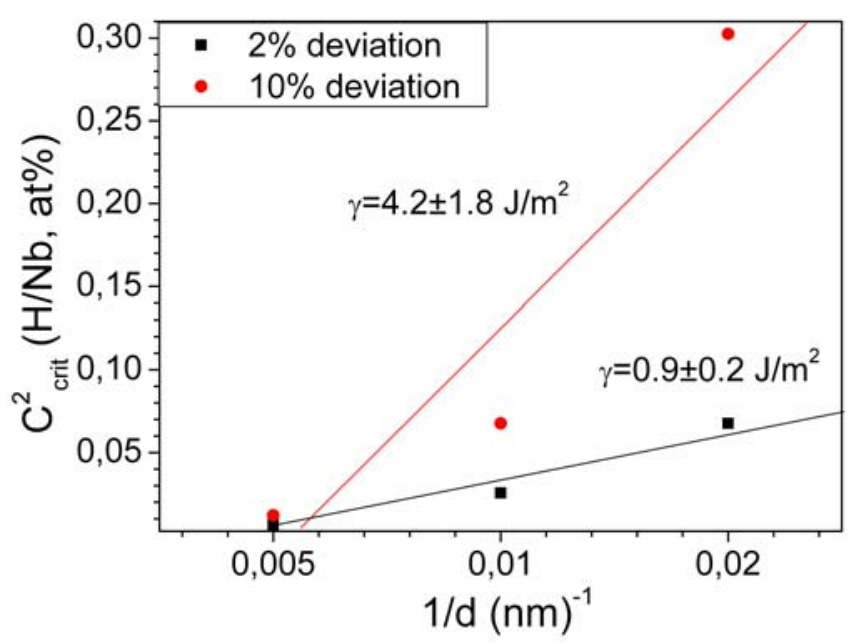

Figure 3.50: $c^{2}{ }_{\text {crit }}$ versus inverse film thickness for $\mathrm{Pd} / \mathrm{Nb} / \mathrm{Pd}$ on $0.5 \mathrm{~mm} \mathrm{PC}$. The critical values for buckling on thinner substrates are larger because of lateral expansion of substrates during hydrogen loading and elastic energy dissipation (see Chap. 3.1.3) leading to large (wrong) adhesion energy values.

The resulting data are summarized in the Tab. 3.5. 
Table 3.5: Critical concentrations for delamination of $P d / N b / P d$ from $0.5 \mathrm{~mm}$ and $1 \mathrm{~mm} P C$ and calculated adhesion energies are shown. Gray shadowed adhesion data were falsified either by substrate thickness effect (stretching) or by taking high critical concentrations at the stress deviation of $10 \%$.

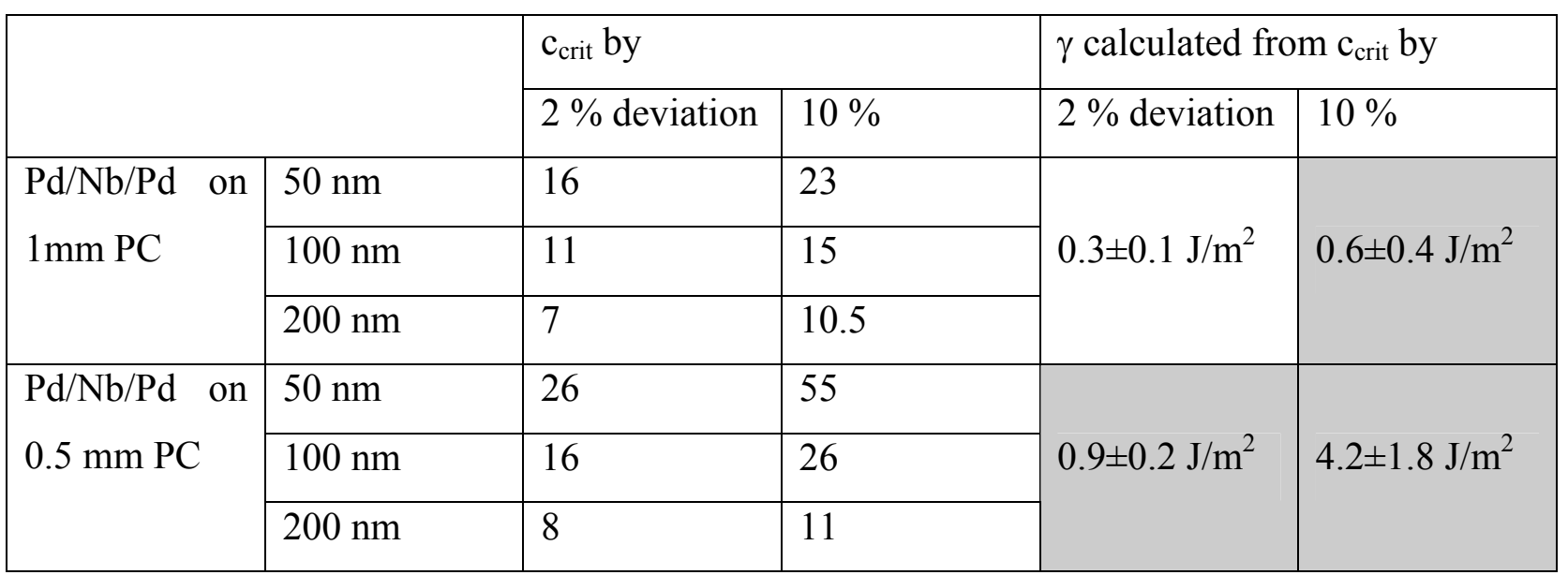

The results by taking the critical H-concentrations at $2 \%$ deviation of the stress curve from linear development is in good accordance with the adhesion energy calculated from the buckle shape (Chap. 3.4.4). On the other hand, the determined adhesion energy value for the $0.5 \mathrm{~mm}$ polycarbonate is higher, than for $1 \mathrm{~mm}$ PC, resulting from the much higher values of the critical hydrogen concentrations for thinner substrates. The reason for that is the straining of substrates as found in Chap.3.1.3. Therefore it can be pointed out that the critical hydrogen concentrations are applicable for the adhesion energy determination only if the substrate is rigid by stress deviation of $2 \%$ which corresponds to critical stress for buckle appearance determined optically. $1 \mathrm{~mm}$ PC substrates were found to act as rigid substrates giving proper results of the adhesion energy.

The adhesion energy values calculated from the critical concentrations at $10 \%$ deviation of the stress are higher than at $2 \%$, as the stress curves for different film thicknesses show different trends of deviating from a linear development after the onset of buckling. The reason for this effects results from the different widths of the buckles. The width of buckles is smaller for thinner substrates and thinner films. This means that the stress curves for the thinner substrates and thinner films are flatter and the $10 \%$ stress deviation corresponds to much higher hydrogen concentrations. This implicates the larger error in the adhesion energy calculation.

The adhesion energy values calculated from the critical stress are similar than those resulting from the critical concentrations. 


\subsubsection{Influence of PC plasma pre-treatment on the adhesion energy values}

To investigate the influence of substrate surface plasma pre-treatment on the adhesion energy, two types of PC-surfaces were prepared before sputtering of $\mathrm{Pd} / \mathrm{Nb} / \mathrm{Pd}$ films. One set of $\mathrm{PC}$ substrates was treated (cleaned) by short dipping it into the isopropanol. Another set was treated with an AC-Ar-plasma at 0.3 mbar for 10 min to enhance the adhesion. This pre-treatment has been performed by the group of Dr. Guido Grundmeier (Max-Planck Institut für Eisenforschung, Düsseldorf). Stress measurements on $\mathrm{Pd} / \mathrm{Nd} / \mathrm{Pd}$ films with $100 \mathrm{~nm}$ and $200 \mathrm{~nm} \mathrm{Nb}$ layers with 10 nm Pd-layers on both sides were carried out. In Fig. 3.51 the stress curves resulting from stepwise hydrogen loading of $\mathrm{Pd} / \mathrm{Nb} / \mathrm{Pd}$ films with $100 \mathrm{~nm}$ Nb-layers on differently pre-treated $1 \mathrm{~mm}$ PC substrates are plotted.

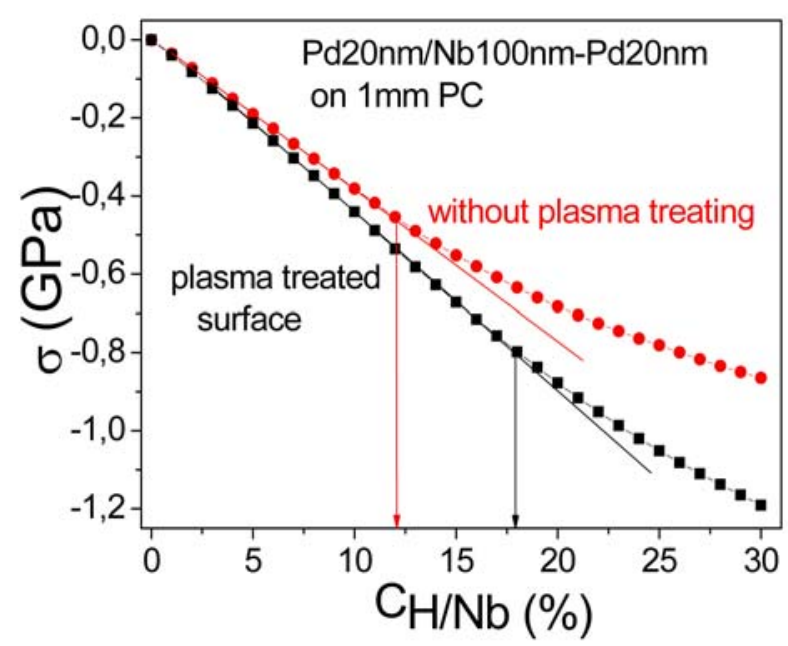

Figure 3.51: Stress evolution in stepwise H-loaded Pd(10nm)/Nb(100nm)/Pd(10nm)//PC (1mm) samples that have been simultaneously sputtered onto differently pre-treated PC-surfaces.

The critical hydrogen concentration for buckling was determined at the point where the stress deviates $2 \%$ from the linear development. As can be seen, the slope of the stress curve and the critical hydrogen concentration for the film sputtered onto the plasma pre-treated PC is higher than for the film without plasma pre-treatment. The critical H-concentration for the sample without plasma treatment is $12 \% \mathrm{H} / \mathrm{Nb}$ and for the plasma pre-treated surface it is $18 \% \mathrm{H} / \mathrm{Nb}$, while the critical stress is $-0.45 \mathrm{GPa}$ and $-0.8 \mathrm{GPa}$, respectively. This already shows that the adhesion between a metal film and the plasma pre-treated PC is better than that of films on PC substrates cleaned by isopropanol only. The plasma pre-treatment generates a clean and chemically active PC surface that enables the formation of chemical bonds between the metal films and PC, so that the adhesion is enhanced. The surface free energy of the plasma-treated polymer can be increased significantly [W08]. Also, it is assumed that the plasma pre-treated 
surface of PC is softer than before, enabling a deeper penetration of metal atoms into the PC during sputtering. This assumption has to be proved in the future investigations. As a result the metal-polymer bond is stronger and therefore already the initial slope of the stress curve is larger. Similar results are obtained for the $200 \mathrm{~nm} \mathrm{Nb-film,} \mathrm{see} \mathrm{Fig.} \mathrm{3.52.}$

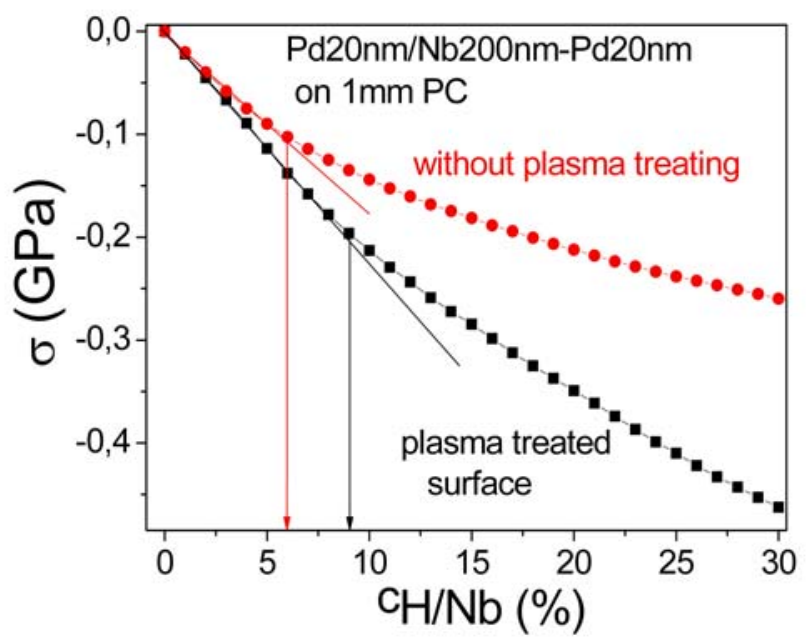

Figure 3.52: Stress evolution in stepwise H-loaded Pd/Nb/Pd (10 nm / $200 \mathrm{~nm} / 10 \mathrm{~nm})$ films on differently pre-treated $P C$-surfaces, sputtered in the same deposition run.

The results of the assigned adhesion energy calculated from the critical values of $\mathrm{H}$-concentration and stress, respectively, are given in Tab. 3.6, which shows the critical stresses and hydrogen concentrations for buckling obtained from these measurements, as well.

Table 3.6: Critical values for buckling by hydrogen loading of $\mathrm{Pd} / \mathrm{Nb} / \mathrm{Pd} / \mathrm{PC}$ samples with different pre-treated PC surfaces and calculated adhesion energies of Pd on PC are shown. The adhesion energies have been calculated applying Eq. (1.33) and Eq. (1.34).

\begin{tabular}{|c|c|c|c|c|c|c|c|c|}
\hline & \multicolumn{4}{|c|}{ Plasma pre-treated surface } & \multicolumn{4}{|c|}{ Surface cleaned with isopropanol } \\
\hline & $\begin{array}{l}\mathrm{c}_{\mathrm{H}, \mathrm{crit}}, \\
(\%)\end{array}$ & $\begin{array}{l}\gamma, \\
\left(\mathrm{J} / \mathrm{m}^{2}\right)\end{array}$ & $\begin{array}{l}\sigma_{\text {crit }}, \\
(\mathrm{GPa})\end{array}$ & $\begin{array}{l}\gamma \\
\left(\mathrm{J} / \mathrm{m}^{2}\right)\end{array}$ & $\begin{array}{l}\mathrm{c}_{\mathrm{H}, \mathrm{crit}} \\
(\%)\end{array}$ & $\begin{array}{l}\gamma, \\
\left(\mathrm{J} / \mathrm{m}^{2}\right)\end{array}$ & $\begin{array}{l}\sigma_{\text {crit }} \\
(\mathrm{GPa})\end{array}$ & $\begin{array}{l}\gamma \\
\left(\mathrm{J} / \mathrm{m}^{2}\right)\end{array}$ \\
\hline $\mathrm{Pd} / 100 \mathrm{~nm} \mathrm{Nb} / \mathrm{Pd}$ & 18 & \multirow{2}{*}{$1 \pm 0.1$} & -0.8 & \multirow{2}{*}{$1.6 \pm 0.15$} & 12 & \multirow{2}{*}{$0.3 \pm 0.03$} & -0.45 & \multirow{2}{*}{$0.5 \pm 0.05$} \\
\hline $\mathrm{Pd} / 200 \mathrm{~nm} \mathrm{Nb} / \mathrm{Pd}$ & 9 & & -0.2 & & 6 & & -0.1 & \\
\hline
\end{tabular}

Summarising the results of hydrogen loading of $\mathrm{Pd} / \mathrm{Nb} / \mathrm{Pd}$ films on differently pre-treated $\mathrm{PC}$ substrates it can be pointed out that the critical stresses and hydrogen concentrations for delamination are higher for the films on plasma pre-treated PC-substrates. The adhesion energies were calculated according to equations (1.33) and (1.34) and the resulting values are similar by using of the critical stresses and the critical hydrogen concentrations. 
3.4.3 Stress measurement during hydrogen loading and adhesion energy calculation for different metals on PC

Thin $\mathrm{Me}(10 \mathrm{~nm}) / \mathrm{Nb}(\mathrm{x} \mathrm{nm}) / \mathrm{Pd}(10 \mathrm{~nm})$ with $\mathrm{x}=50 \mathrm{~nm}, 100 \mathrm{~nm}$ and $200 \mathrm{~nm}$ films were sputterdeposited at room temperature onto polycarbonate substrates of $1 \mathrm{~mm}$ thickness. The metal layers of these thicknesses were found to be closed [Wag05]. Molybdenum, iron, chromium and niobium were the investigated (Me)-layers. The PC-surface was pre-treated by short dipping it into isopropanol. The investigated interface lies between PC and the metal film, see Chap. 3.2.4. In Fig. 3.53 the stress curves of stepwise electrolytic hydrogen charged films with different metal sublayers are shown.

a)

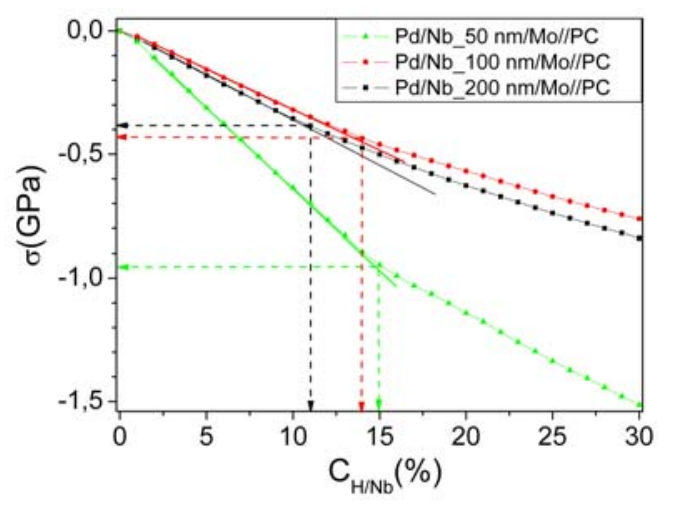

c)

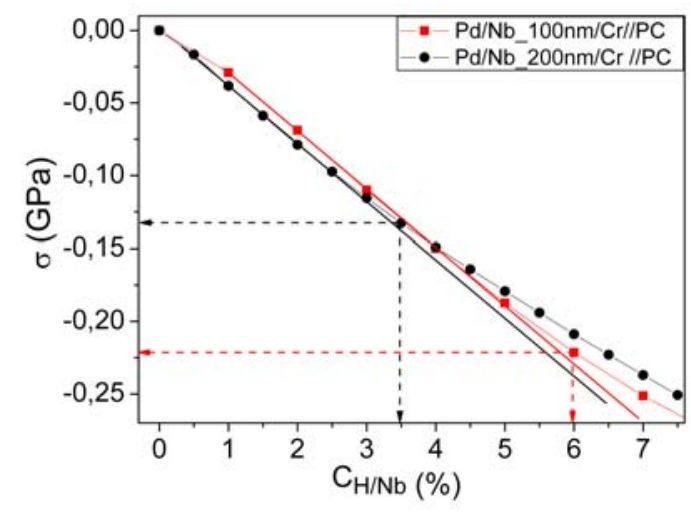

b)

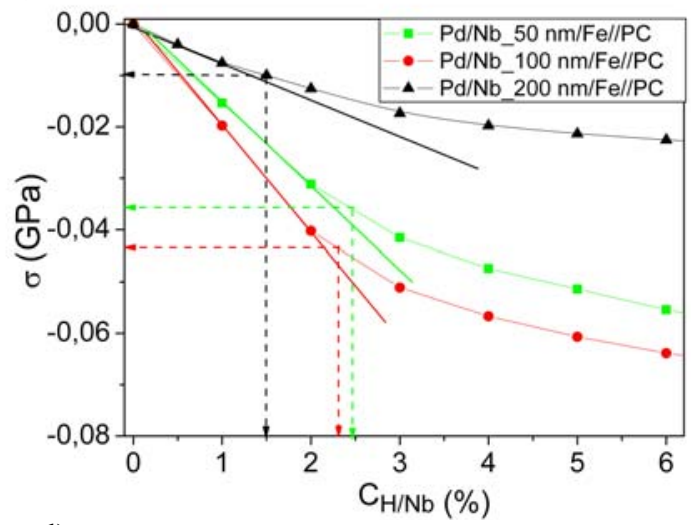

d)

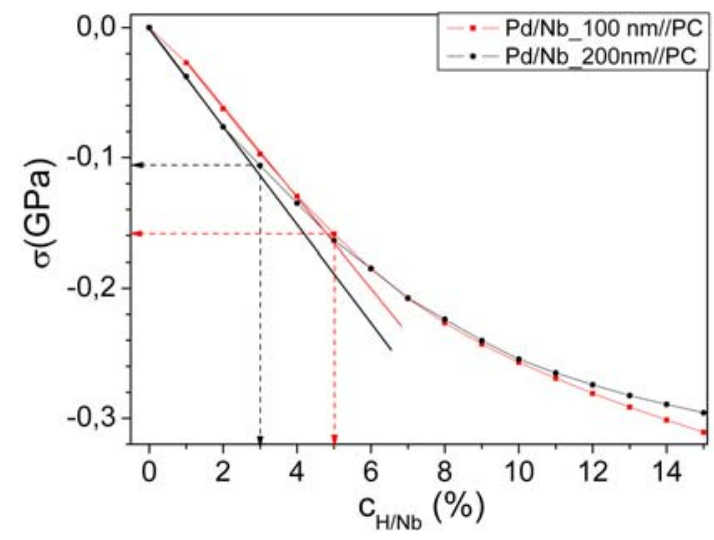

Figure 3.53: Stress curves of $\mathrm{Pd} / \mathrm{Nb} / \mathrm{Me}$ films on PC. Arrows show the critical stresses and hydrogen concentrations for buckling; a) Pd/Nb/Mo//PC, b) Pd/Nb/Fe//PC, c) Pd/Nb/Cr//PC, d) Pd/Nb//PC.

Referring to the critical stresses and hydrogen concentrations obtained from the stress measurements for $2 \%$ deviation of stress from the linear development, adhesion energies can be calculated applying equations (1.34) and (1.33). The data underlying the adhesion energy calculations are plotted in Figs. 3.54 and 3.55. Thereby it has to be taken into account with the exception of molybdenum; other metals (iron, chromium and niobium) oxidize at the interface to the PC surface. This was proved by SIMS analysis of a $\mathrm{Pd} / \mathrm{Nb} / \mathrm{Cr} / / \mathrm{PC}$ sample, see below. 


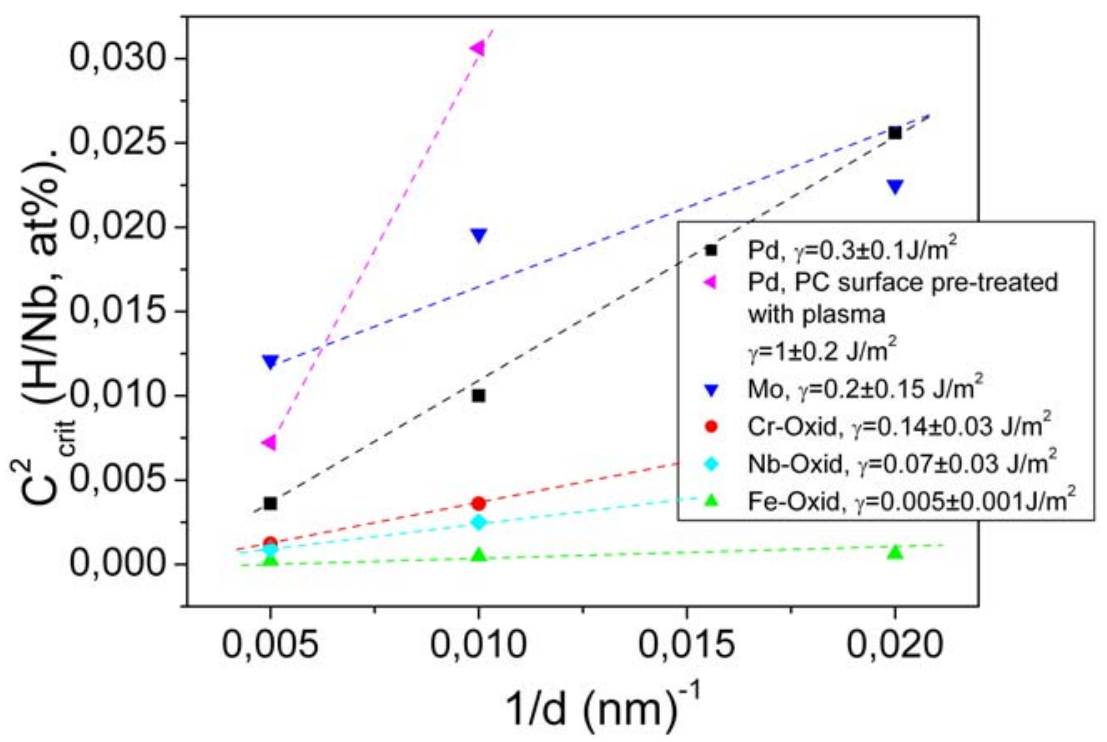

Figure 3.54: $c^{2}{ }_{\text {crit }}$ versus inverse film thickness for $\mathrm{Me} / \mathrm{Nb} / \mathrm{Pd}$ on $1 \mathrm{~mm}$ PC. Resulting adhesion energies $\gamma$ calculated from the critical $\mathrm{H}$-concentrations are given in the label.

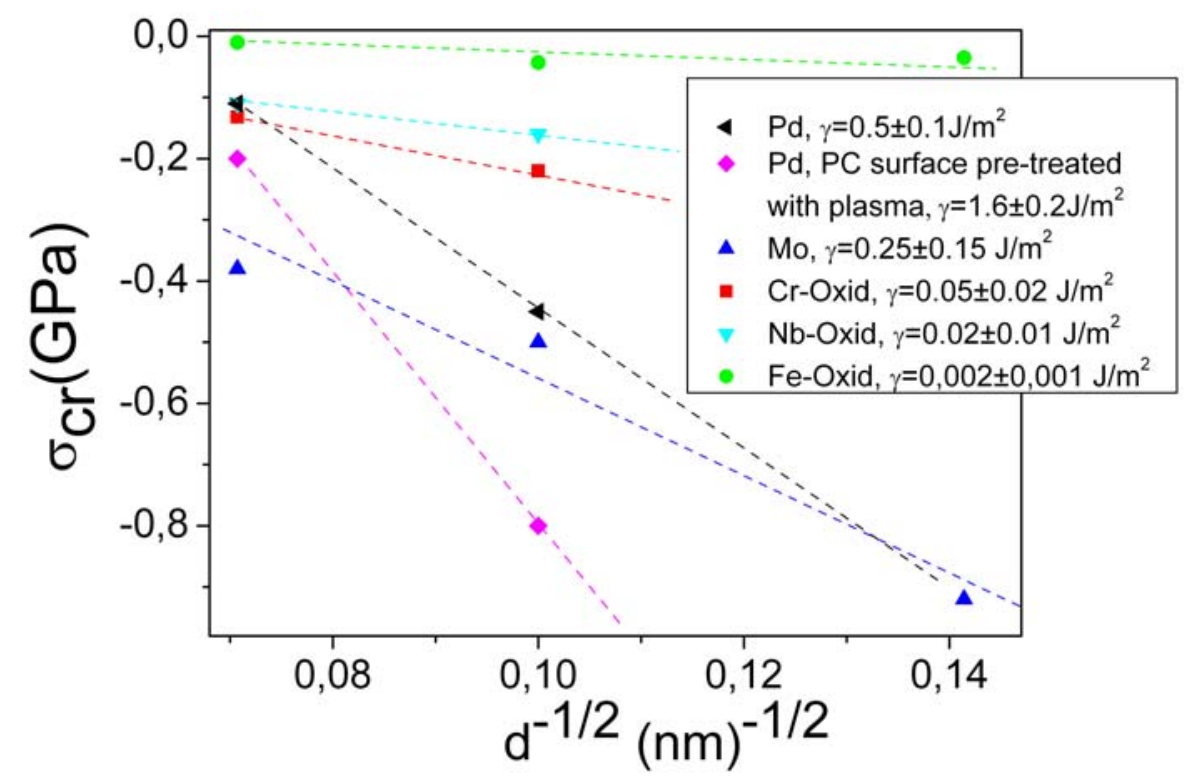

Figure 3.55: $\sigma_{\text {crit }}$ versus inverse film thickness for $\mathrm{Me} / \mathrm{Nb} / \mathrm{Pd}$ on $1 \mathrm{~mm}$ PC. Resulting adhesion energies $\gamma$ calculated from the critical stresses are listed as well.

The resulting adhesion energies for the different metal layers on PC substrates are summarized in Tab. 3.7. 
3. Experimental results

Table 3.7: Adhesion energies of different materials on PC, calculated from the critical H-concentration and the critical stress, respectively. Pd* means Pd deposited onto plasma pre-treated PC surface.

\begin{tabular}{|l|l|l|l|l|l|l|}
\hline Metal & Fe-oxide & Nb-oxide & Cr-oxide & Mo & Pd & Pd * \\
\hline $\begin{array}{l}\gamma\left(\mathrm{c}_{\text {crit }}\right), \\
\left(\mathrm{J} / \mathrm{m}^{2}\right)\end{array}$ & $0.005 \pm 0.001$ & $0.07 \pm 0.03$ & $0.14 \pm 0.03$ & $0.2 \pm 0.15$ & $0.3 \pm 0.1$ & $1 \pm 0.2$ \\
\hline $\begin{array}{l}\gamma\left(\sigma_{\text {crit }}\right), \\
\left(\mathrm{J} / \mathrm{m}^{2}\right)\end{array}$ & $0.002 \pm 0.001$ & $0.02 \pm 0.01$ & $0.05 \pm 0.02$ & $0.25 \pm 0.15$ & $0.5 \pm 0.1$ & $1.6 \pm 0.2$ \\
\hline
\end{tabular}

From Tab. 3.7 it can be extracted that the adhesion energy values for investigated metal oxides are by one order of magnitude lower than those obtained for pure metals. Further, the adhesion energy value of Pd on plasma pre-treated PC is largest.

The results of the SIMS analysis of a $\mathrm{Pd} / \mathrm{Nb} / \mathrm{Cr} / / \mathrm{PC}$ sample concerning the interface oxidation are shown in Fig. 3.56.

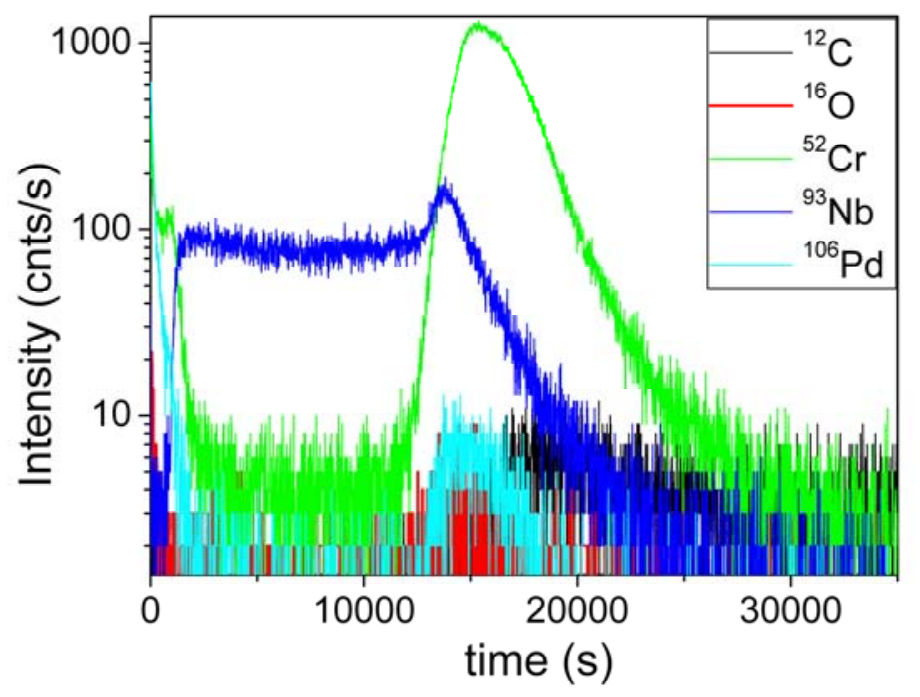

Figure 3.56: SIMS profile of $\mathrm{Pd} / \mathrm{Nb} / \mathrm{Cr} / \mathrm{PC}$ sample. Oxygen enrichment in the Cr-layer (green line) can be seen at the PC surface, which is visualized by the carbon (black line).

In this figure the presence of oxygen, which is plotted as a red line at about $15000 \mathrm{~s}$, in the Crlayer (green line) is proven. PC is detected via its carbon atoms (black lines up to $17000 \mathrm{~s}$ ). This means that the Cr-layer oxidizes at the PC surface. As the Cr-layer was prepared in UHV the oxygen enters the sample after exposing samples to air. As the solubility and mobility of oxygen molecules in PC is large enough, the oxygen is provided by permeation through the polymer. Noble metals like Pd are not oxidized and, therefore, a polymer/metal interface is maintained. Metals which oxidize at the polymer surface are characterized with low adhesion to the substrates, as the oxidation of the metal film alters the nature of the chemical bonds at the interface. 
The difference in adhesion values for metal oxides for adhesion energy calculation from critical stress and critical hydrogen concentrations is about one order of magnitude. Electrochemical hydrogen loading of the films with low adhesion has to be performed by very small loading steps for better determination of critical values for film buckling.

The reliability of the evaluated adhesion energy can be remarkably improved measuring the initial stresses in the films, e.g., by measuring substrate bending during film deposition. In this case the problem of the effect of a thickness dependent initial stress and/or the effect of a remaining compressive stress in the delaminated film can be solved. Those measurements should be performed in the future to improve the adhesion results.

\subsubsection{Adhesion energy measurements by means of white-light interferometry}

Buckle shapes of the $\mathrm{Pd} / \mathrm{Nb} / \mathrm{Pd} / / \mathrm{PC}$ packages were measured using a white-light interferometer. Images of buckles were obtained right at the critical concentration, which was $7 \% \mathrm{H} / \mathrm{Nb}$ for 200 $\mathrm{nm} \mathrm{Nb}, 10 \% \mathrm{H} / \mathrm{Nb}$ for $100 \mathrm{~nm}$ and $16 \% \mathrm{H} / \mathrm{Nb}$ for $50 \mathrm{~nm} \mathrm{Nb}$ with the focus on straight sided buckles. These critical concentrations were determined during hydrogen loading of other similar samples.

A $\mathrm{Pd} / \mathrm{Nb} / \mathrm{Pd}$ film with $100 \mathrm{~nm}$ Nb-layer was loaded until $10 \% \mathrm{H} / \mathrm{Nb}$. In Fig. 3.57 the 3-Dvisualisation of two different straight-sided buckles of this film in height and colour scales are shown.

a)

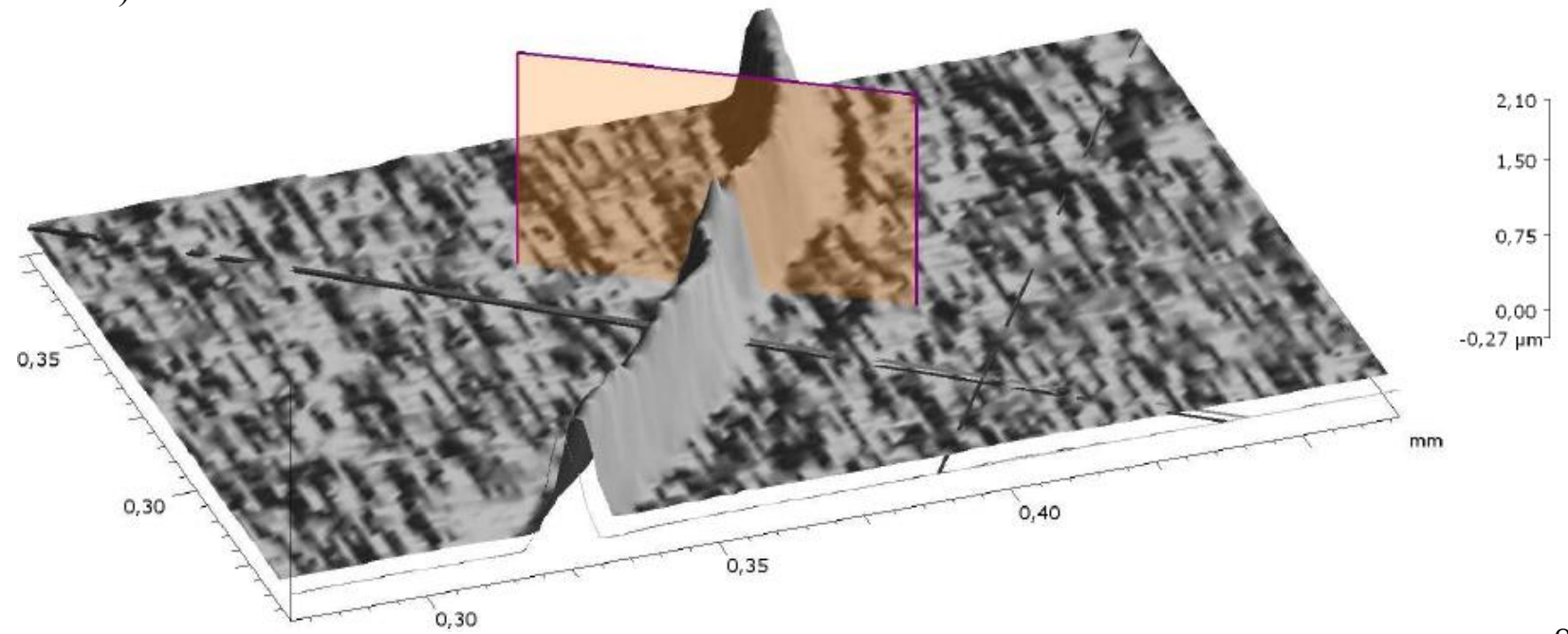




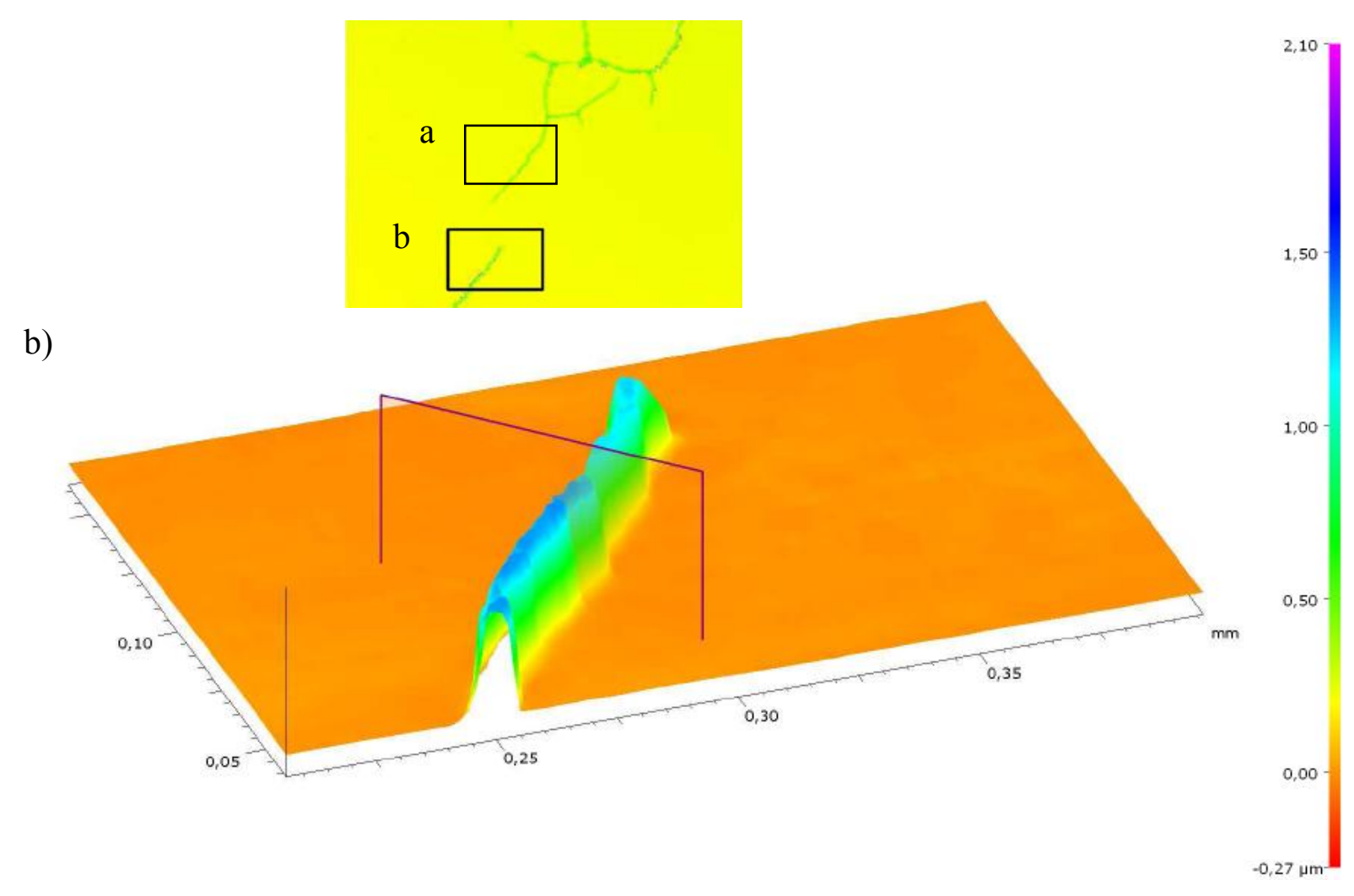

Figure 3.57: a) 3-D-visualisation of a straight-sided buckle of a $\mathrm{Pd} / \mathrm{Nb}(100 \mathrm{~nm}) / \mathrm{Pd} / \mathrm{PC}$ sample, as measured using white-light interferometry $(10 \% \mathrm{H} / \mathrm{Nb})$. A buckle width $\mathrm{I}=12.37 \mu \mathrm{m}$ and a buckle height $h=0.96 \mu \mathrm{m}$ is determined. b) 3-D-visualisation in colour scale of a straight-sided buckle. A buckle width $l=12.37 \mu \mathrm{m}$ and $a$ buckle height $h=1.31 \mu \mathrm{m}$ is determined. The top view of the sample surface is shown in the middle of the figure. Rectangles in the top view show the measured areas.

These buckles have a small height variation and their cross-section is close to the sinusoidal one. The calculation of the adhesion energy for this film from the buckle geometry using Gille and Rau theory gives $\gamma=1.05( \pm 0.45) \mathrm{J} / \mathrm{m}^{2}$. This value is in a good agreement with the value obtained by the controlled-buckling technique, $\gamma=0.4( \pm 0.1) \mathrm{J} / \mathrm{m}^{2}$. The error by measuring with the optical interferometer is $1.5 \mu \mathrm{m}$ for lateral displacements and $0.01 \mu \mathrm{m}$ for the height measurements.

The image of a $20 \% \mathrm{H} / \mathrm{Nb}$ loaded film is shown in Fig. 3.58. The large variations in the height of buckles can be seen, which are caused as was assumed by plastic deformations. 


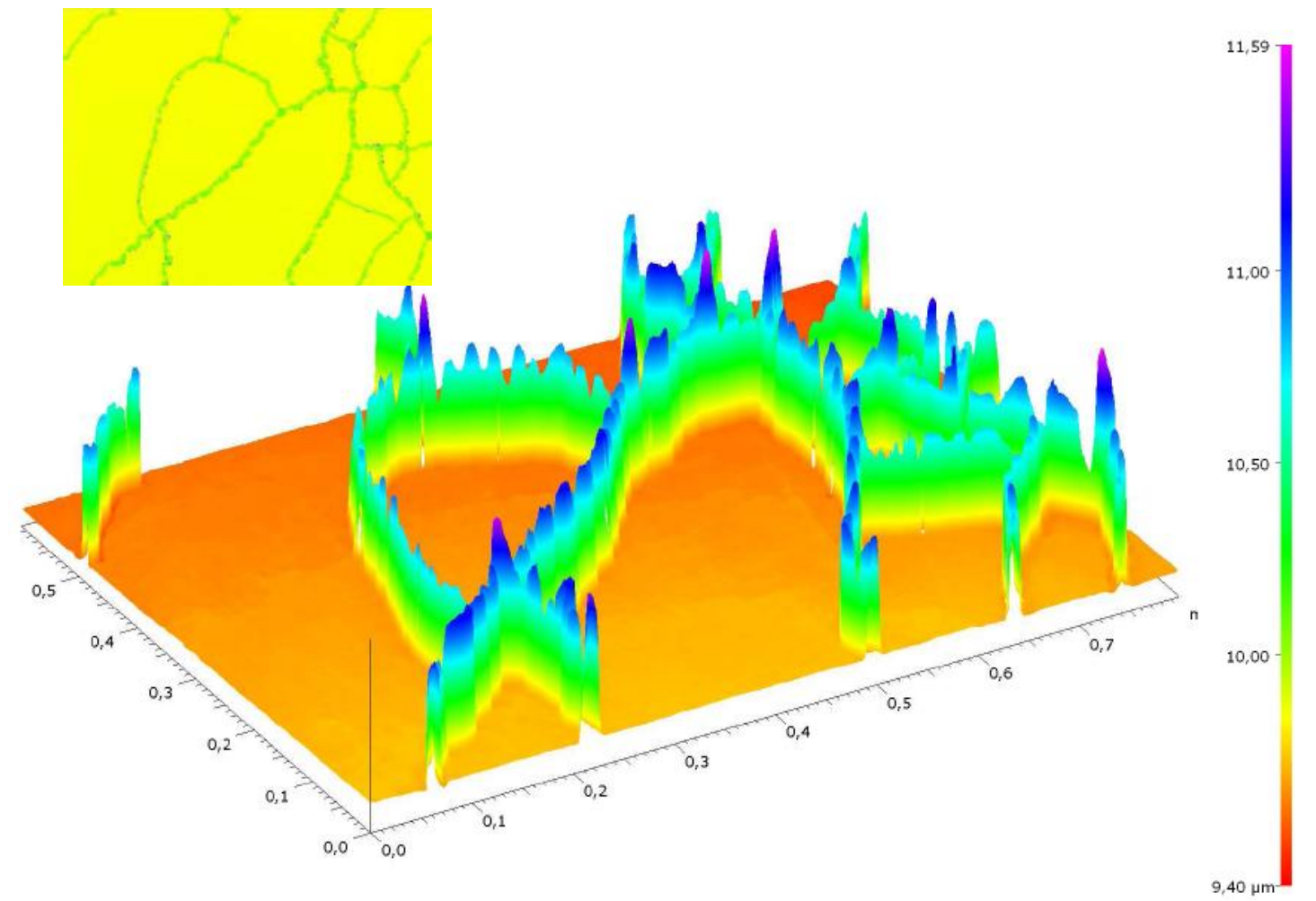

Figure 3.58: 3-D-visualisation in colour scale of a buckled surface of a $P d / N b(100 \mathrm{~nm}) / \mathrm{Pd} / / \mathrm{PC}$ package, as measured using white-light interferometry $(20 \% \mathrm{H} / \mathrm{Nb})$. The large variation in the height of the buckles is assumed to result from a plastic deformation of the buckles.

The surface of a typical straight-sided buckle (the bold black line follows the straight side) of a 7 \% loaded $\mathrm{Pd} / \mathrm{Nb} / \mathrm{Pd}$ film with $200 \mathrm{~nm} \mathrm{Nb}$ is shown in 3-D-visualisation in Fig. 3.59. The crosssectional shape of the buckle is close to the ideal one. A buckle width of about $16 \mu \mathrm{m}$ is obtained. The buckle height varies between $1 \mu \mathrm{m}$ and $2 \mu \mathrm{m}$.

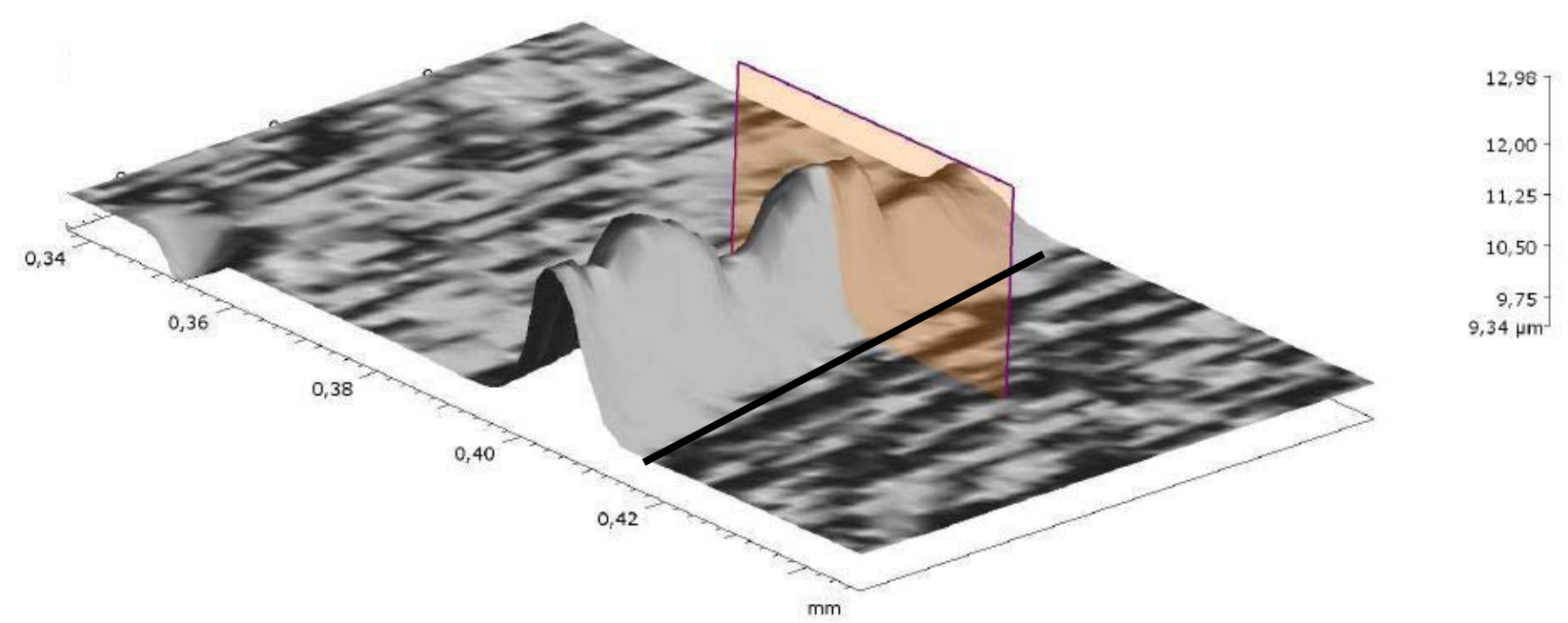

Figure 3.59: 3-D-visualisation of a straight-sided buckle of a Pd/Nb(200 nm)/Pd/PC sample at 7\% $\mathrm{H} / \mathrm{Nb}$, as measured using white-light interferometry. The cross-sectional form of the buckle is close to the sinusoidal form. A buckle width of about $16 \mu \mathrm{m}$ is obtained. The buckle height varies between $1 \mu \mathrm{m}$ and $2 \mu \mathrm{m}$. 
The height variation is quite large, reaching about $100 \%$ of the minimum height. This effect results in large variations of calculated adhesion energies using Gille und Rau equation (2.3). Taking $l=16.0( \pm 1.5) \mu \mathrm{m}$ and $\mathrm{h}=1.99( \pm 0.01) \mu \mathrm{m}$ an adhesion energy of $\gamma=8.7( \pm 4.1) \mathrm{J} / \mathrm{m}^{2}$ results. Taking a cross section in the minimum region with $l=16.0( \pm 1.5) \mu \mathrm{m}$ and $\mathrm{h}=1.22$ $( \pm 0.01) \mu \mathrm{m}$ gives $\gamma=1.4( \pm 0.6) \mathrm{J} / \mathrm{m}^{2}$. The variation in the calculated adhesion energies $\gamma$ is large because the term $h / l$ contributes with an exponent of four to the result of Eq. 2.3. This problem arises because the experimental buckle morphologies can be much more complicated than the sample geometries assumed in the model. But, the obtained adhesion energy for the minimum buckle height, $\gamma=1.4( \pm 0.6) \mathrm{J} / \mathrm{m}^{2}$ is in agreement with the value obtained by the controlledbuckling technique. Thus, consistent results can be obtained when plastic deformation does not affect the morphology measurements. The buckle shape in Fig. 3.59 is very similar to the varicose mode shown in Fig. 1.7, which is the transition mode between the Euler mode and the telephone cord mode of buckles. Further measurements are needed taking more surface pictures after small hydrogen loading steps to investigate the phenomena of modes transition and buckles propagation. The transformation of the varicose mode in to the telephone cord mode at higher hydrogen concentrations for this sample (see Fig. 3.62) was not observed.

3-D-images of a film with $50 \mathrm{~nm} \mathrm{Nb}$ - layer loaded until $16 \% \mathrm{H} / \mathrm{Nb}$ are shown in Figs. 3.60 and 3.61 .

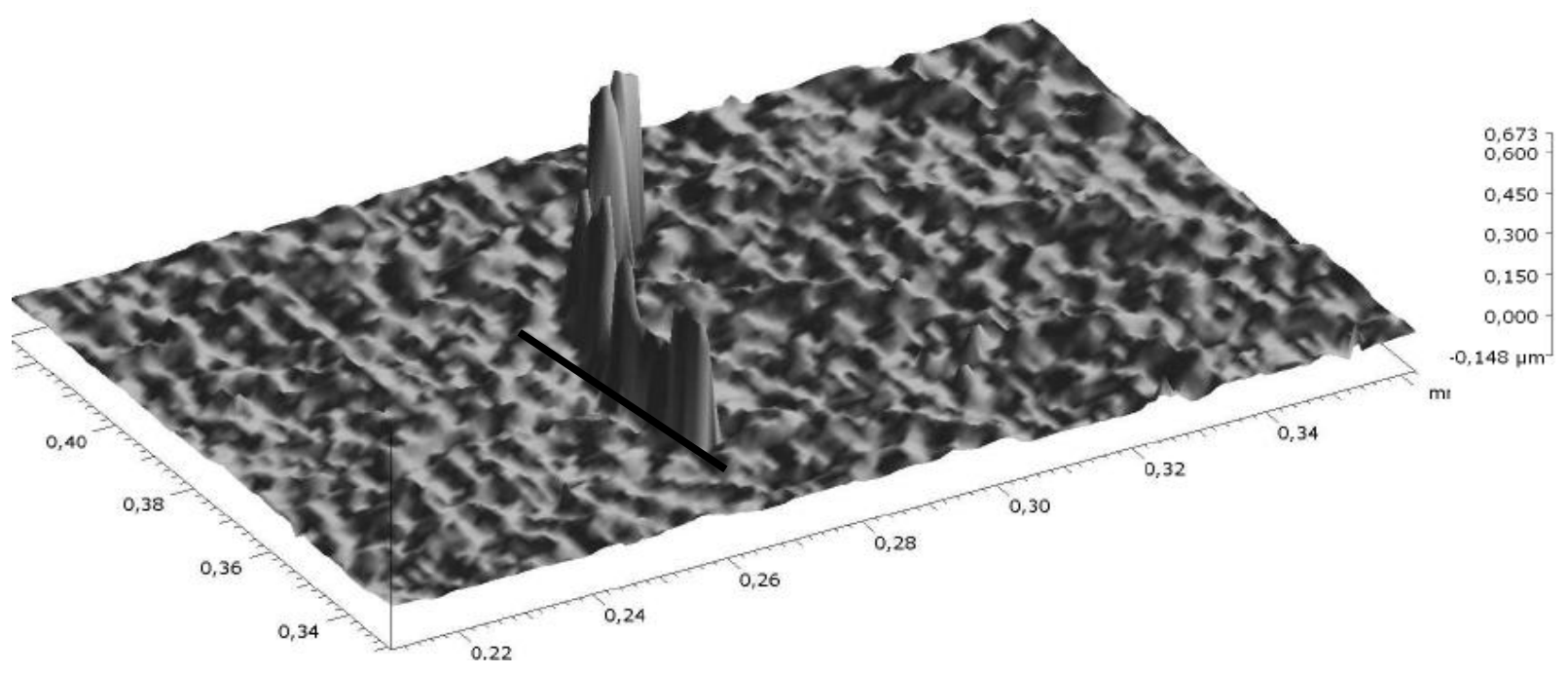

Figure 3.60: 3-D-visualisation of a slightly bended, straight-sided buckle of a $\mathrm{Pd} / \mathrm{Nb}(50 \mathrm{~nm}) / \mathrm{Pd} / / \mathrm{PC}$ sample at $16 \% \mathrm{H} / \mathrm{Nb}$, as measured using a white-light interferometer. A buckle width of about $9 \mu \mathrm{m}$ is obtained. The buckle height varies between $0.4 \mu \mathrm{m}$ and $0.7 \mu \mathrm{m}$. 


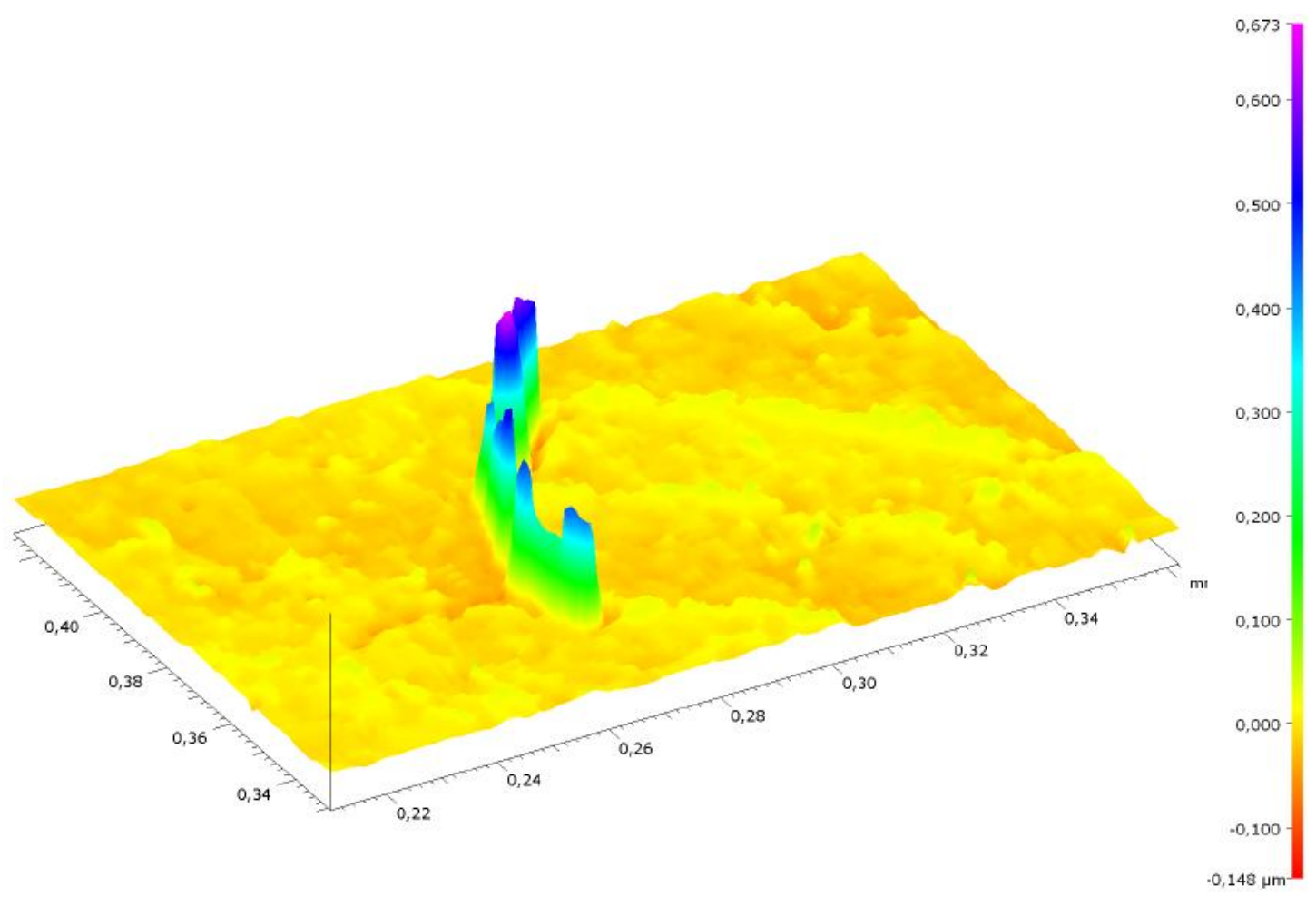

Figure 3.61: Surface 3-D-visualisation in colour scale of a slightly bended, straight-sided buckle (the same than in Fig. 3.60) of a $\mathrm{Pd} / \mathrm{Nb}(50 \mathrm{~nm}) / \mathrm{Pd} / \mathrm{PC}$ sample at $16 \% \mathrm{H} / \mathrm{Nb}$, as measured using a whitelight interferometer.

The buckle height varies between 0.4 and $0.7 \mu \mathrm{m}$. Taking a cross section in the minimum region with $l=9 \mu \mathrm{m}$ and $h=0.4 \mu \mathrm{m}$, the adhesion energy follows as $\gamma=0.05( \pm 0.03) \mathrm{J} / \mathrm{m}^{2}$. Such very small adhesion energy values can be explained by influence of the Pd layers on the buckle formation. For $50 \mathrm{~nm} \mathrm{Nb}$ layer the influence of the $20 \mathrm{~nm}$ Pd layers on both sides is much more significant than for thicker $\mathrm{Nb}$ layers. This influence results in a reduction of the buckle height and in the smaller adhesion values.

Other images of the $\mathrm{Pd} / \mathrm{Nb}(200 \mathrm{~nm}) / \mathrm{Pd} / / \mathrm{PC}$ package show that the elevated regions of the buckles act as growth centres for new buckles. These grow almost perpendicular to the original buckles direction to minimize elastic energy in two dimensions. This is illustrated in Fig. 3.62. New buckles are mainly straight sided and of constant height. Their height is similar to the minimum height of the buckles in Fig. 3.59. Thus, it can be assumed that the minimum buckle height value is approaching the initial height of the straight-sided buckle. The strong height increase of about $100 \%$ in some areas was interpreted as resulting from plastic deformation and distortion of the buckle geometry from the straight-sided buckle form with a constant height. Plastic deformation is assumed to strongly change the local morphology of the buckle. 


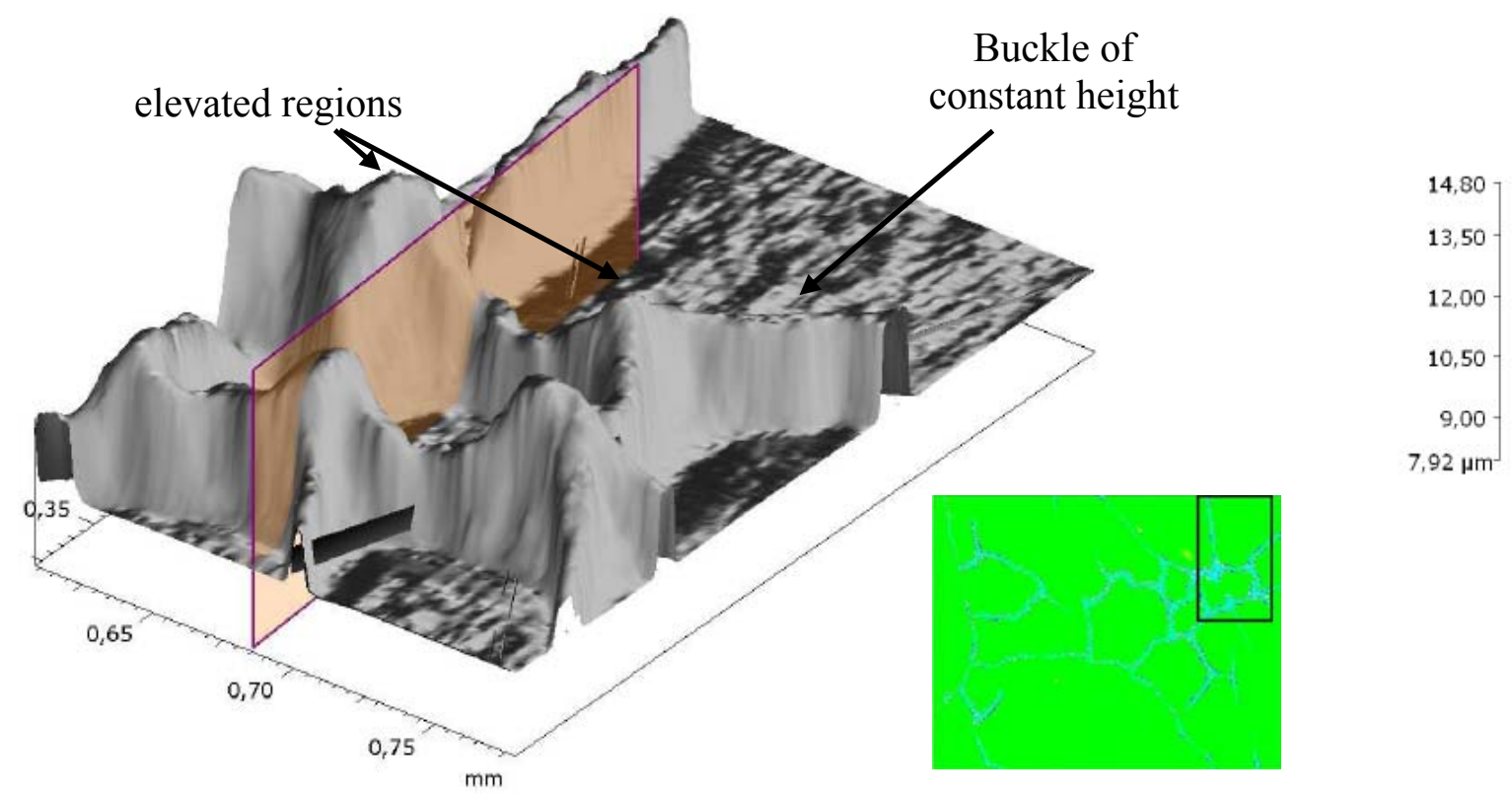

Figure 3.62: 3-D-visualisation of a buckle region of a $\mathrm{Pd} / \mathrm{Nb}(200 \mathrm{~nm}) / \mathrm{Pd} / \mathrm{PC}$ sample at about $7 \%$ $\mathrm{H} / \mathrm{Nb}$ obtained using a white-light interferometer. Locally large height areas of buckles act as centres for new buckles evolving in perpendicular directions. Locally low height areas have comparable height to those buckles, which just have been formed. The large buckle heights are interpreted with plastically deformed buckles (down right: top view of the sample and the measured segment in the black rectangle).

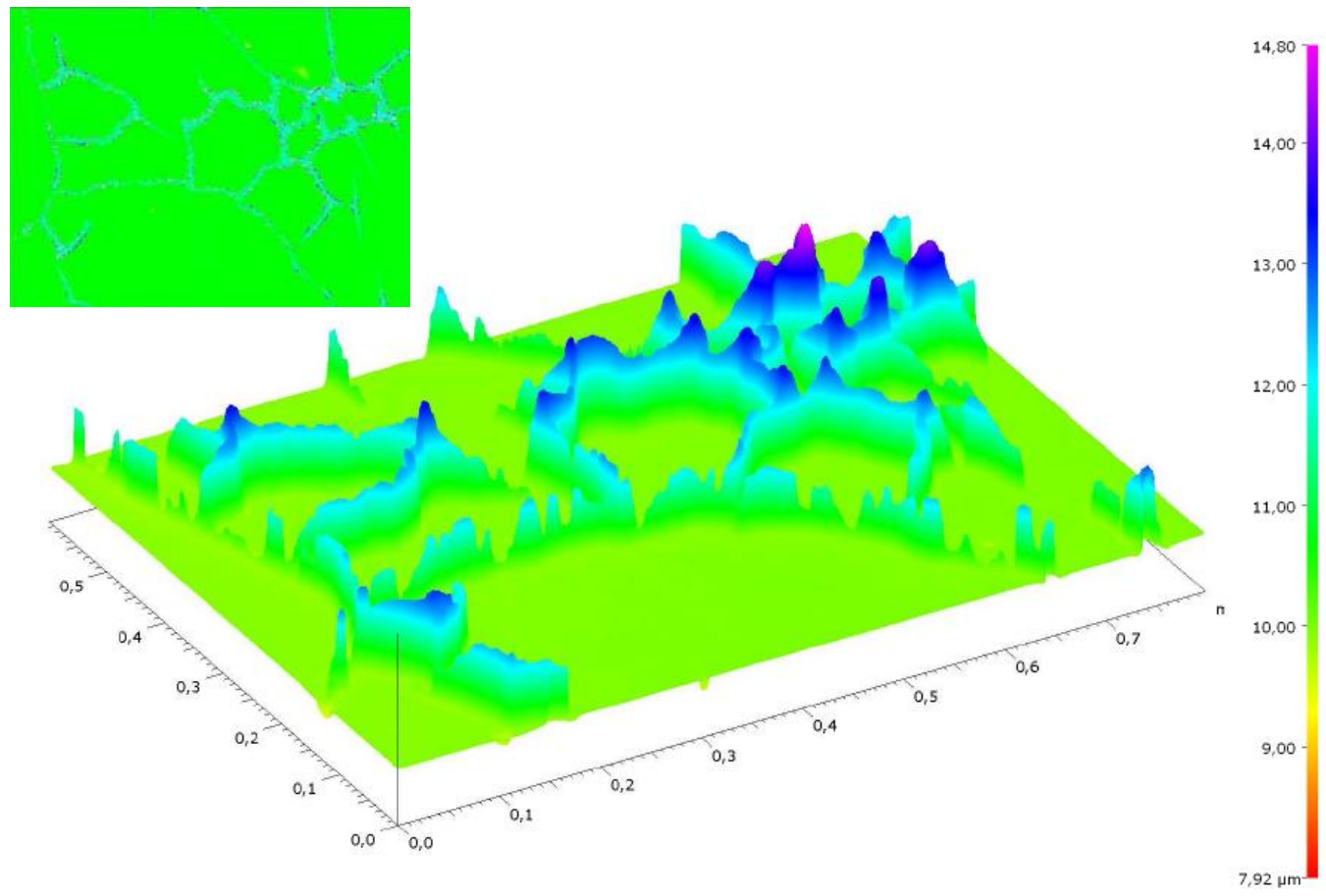

Figure 3.63: 3-D-visualisation of the $\mathrm{Pd} / \mathrm{Nb}(200 \mathrm{~nm}) / \mathrm{Pd} / \mathrm{PC}$ sample at about $0.07 \mathrm{H} / \mathrm{Nb}$, as measured using a white-light interferometer. The maximal height difference is about $4.3 \mu \mathrm{m}$ (top left: top view of the sample and the measured segment). 
It can be assumed that buckle morphologies obtained by hydrogen loading are different from those obtained by using other methods, e.g. by developing of thermal stress between film and substrate during sample cooling. These intrinsic buckles occur during film deposition when the substrate is not artificially cooled. The local sample temperature is not known in this case. 3-D images of intrinsic buckles that resulted from such thermal stress between film and substrate for the $\mathrm{Pd} / \mathrm{Nb}(200 \mathrm{~nm}) / \mathrm{Pd} / / \mathrm{PC}$ package have been analysed as well. By the surface observation of the unloaded film with $200 \mathrm{~nm}$ Nb-layer intrinsic buckles were found. One 3-D-visualisation of such intrinsic straight-sided buckle is shown in Fig. 3.64.

a)
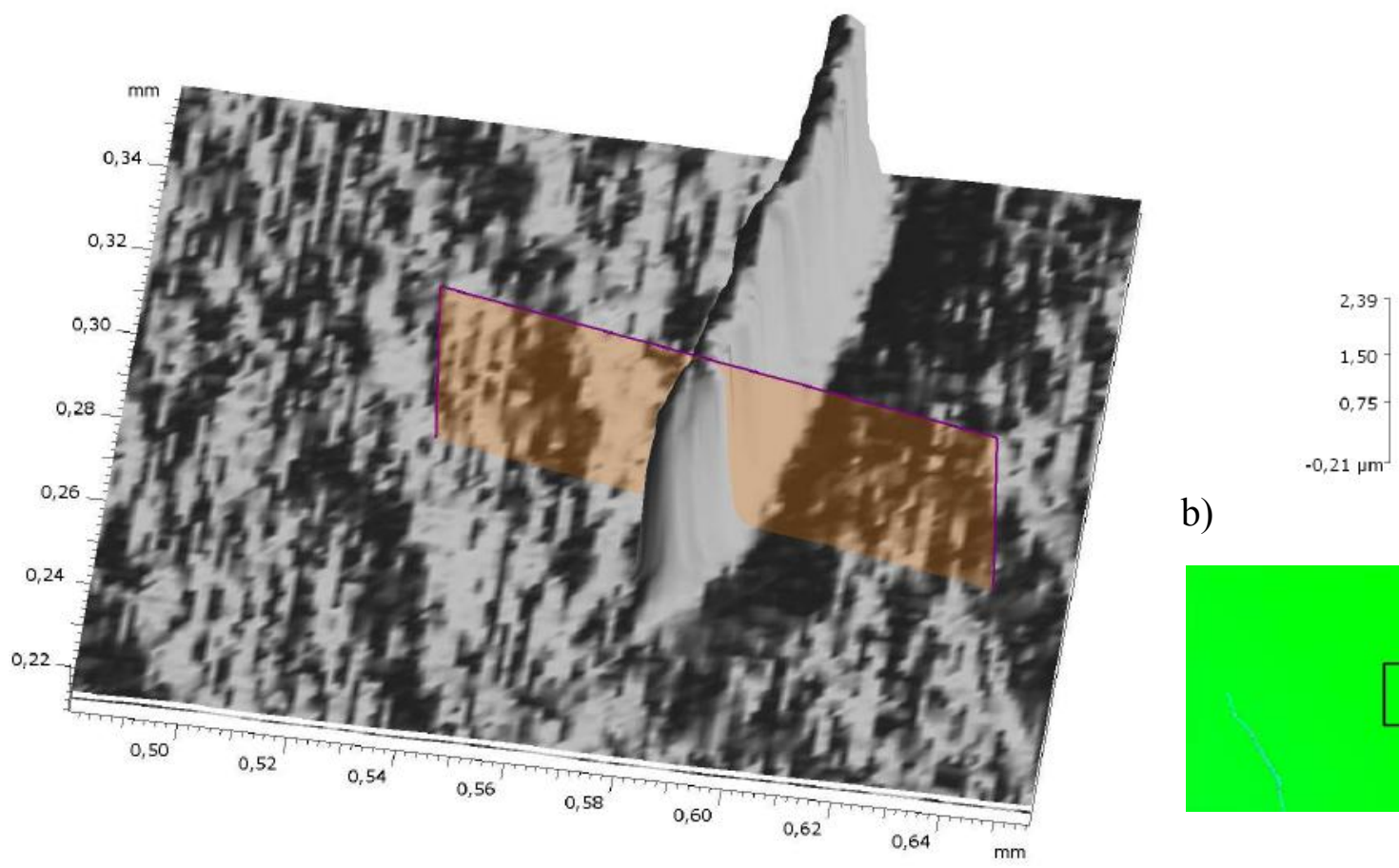

b)

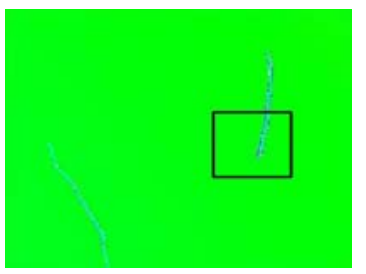

Figure 3.64: a) 3-D-visualisation of a straight-sided intrinsic buckle of a $\mathbf{P d} / \mathrm{Nb}(200 \mathrm{~nm}) / \mathbf{P d} / \mathbf{P C}$ sample, as measured using white-light interferometer (without hydrogen loading). A buckle width $I=$ $14.1 \mu \mathrm{m}$ and a buckle height $h=2.42 \mu \mathrm{m}$ is determined. b) The top view of the sample surface with selected area for buckle visualisation.

It can be seen that only small buckle height variations are present for intrinsic buckles, but they are not as large as after hydrogen loading of the $\mathrm{Nb}$-layer.

The height of this intrinsic buckle is much larger than the buckle in Fig. 3.59. Taking buckle height $h$ and width $l$ of the intrinsic buckle (see Fig. 3.64) and implementing this in equation 2.3 gives $\gamma_{\mathrm{Pd} / \mathrm{PC}}=31( \pm 18) \mathrm{J} / \mathrm{m}^{2}$ which is far above the values obtained before. Also, this value is unexpectedly large and inconsistent to the values discussed before. The origin of this effect can be explained by plastic deformation. Plastic deformation modifies the buckle's morphology apart from the ideal morphology. The plastic deformations are not implemented in the formula of Gille and Rau. Adhesion energy calculated from the geometry of plastically deformed buckle is larger, 
as if the critical stress for buckling is higher. Thus, also intrinsic buckles can be affected by plastic deformation and, in this case, application of Eq. 2.3 gives wrong results.

In Tab. 3.8 the data obtained for buckles of three different film thicknesses are summarized. For the $200 \mathrm{~nm}$ film the height and the width of buckles for different hydrogen concentrations are determined as well as those of intrinsic buckles. Grey shaded columns are interpreted as those resulting from plastically deformed buckles. The adhesion energies shown in Tab. 3.8 are smaller for thinner films. The reason for that might be the influence of the Pd top layer on the thinner films resulting in a reduction of the buckle height.

Table 3.8: Buckle heights $h$ and lengths $l$, and the resulting adhesion energies $\gamma$ for the Pd/PC interface obtained by the theory of Gille and Rau. Adhesion energies resulting from the geometry of plastic deformed buckles are shown in grey shaded columns.

\begin{tabular}{|c||c||c|c|c|}
\hline film thickness & $\begin{array}{c}\text { Hydrogen } \\
\text { concentration } \\
\text { in a metal } \\
\text { film }\end{array}$ & $\begin{array}{c}\mathrm{h}[\mu \mathrm{m}] \\
( \pm 10 \mathrm{~nm})\end{array}$ & $\begin{array}{c}1[\mu \mathrm{m}] \\
( \pm 1,5 \mu \mathrm{m})\end{array}$ & $\gamma\left[\mathrm{J} / \mathrm{m}^{2}\right]$ \\
\hline $200 \mathrm{~nm}$ & $7 \mathrm{at} \% \mathrm{H} / \mathrm{Nb}$ & 1.22 & 15.8 & $1.4 \pm 0.6$ \\
\hline & $15 \mathrm{at} \% \mathrm{H} / \mathrm{Nb}$ & 2.42 & 24.8 & $3.3 \pm 0.9$ \\
\hline $100 \mathrm{~nm}$ & $10 \mathrm{at} \% \mathrm{H} / \mathrm{Nb}$ & 1.04 & 14.1 & $1.05 \pm 0.45$ \\
\hline $50 \mathrm{~nm}$ & $16 \mathrm{at} \% \mathrm{H} / \mathrm{Nb}$ & 0.4 & 9.0 & $0.05 \pm 0.03$ \\
\hline
\end{tabular}

To summarize, this method gives good results as long as buckles are of uniform height and plastic deformation of the buckles can be excluded. For the Pd/PC-interface the adhesion energies $\gamma=1.05( \pm 0.45) \mathrm{J} / \mathrm{m}^{2}$ and $\gamma=1.4( \pm 0.6) \mathrm{J} / \mathrm{m}^{2}$, obtained for $100 \mathrm{~nm} \mathrm{Nb}$ layer and for 200 $\mathrm{nm} \mathrm{Nb}$ layer at the critical concentrations are in good accordance with the value obtained by the controlled-buckling technique $\gamma=0.4( \pm 0.1) \mathrm{J} / \mathrm{m}^{2}$. The similarity of the results obtained using both methods allows a conclusion about the correctness of the calculated adhesion energies. However, there remains the problem if in real buckles plastic deformations can be excluded completely.

It was also shown that large height variations up to $100 \%$ of the original buckle height appear at the top of the buckle. These height variations were interpreted to result from local plastic deformation. If a plastically deformed buckle is measured, adhesion energies can be largely overestimated when the Gille-and-Rau theory is applied. Therefore, the total 3-D visualisation of 
the buckle morphology is important when adhesion energies are determined using the Gille-andRau theory.

To conclude, complicated buckle morphologies with large height variations even for straightsided buckles were observed using white light interferometer. The diversity of buckle morphologies and the content of plastic deformed regions might be reasons for the large variety of measured adhesion energies.

\subsubsection{Comparative measurements at the model system $\mathrm{Pd} / \mathrm{Nb} / \mathrm{Pd} / / \mathrm{PC}$ by} determination of the peel strength

In cooperation with the group of Prof. F. Faupel, University of Kiel, peel-of measurements at certain model samples were performed.

The peel-off strength derived from the different measurements shows a small dispersion, as it is seen in Fig 3.65. This demonstrates a good reproducibility of the adhesive properties of our layers. However, it is not possible to directly calculate the adhesion energy from the peel strength. Therefore only qualitative conclusions can be drawn.
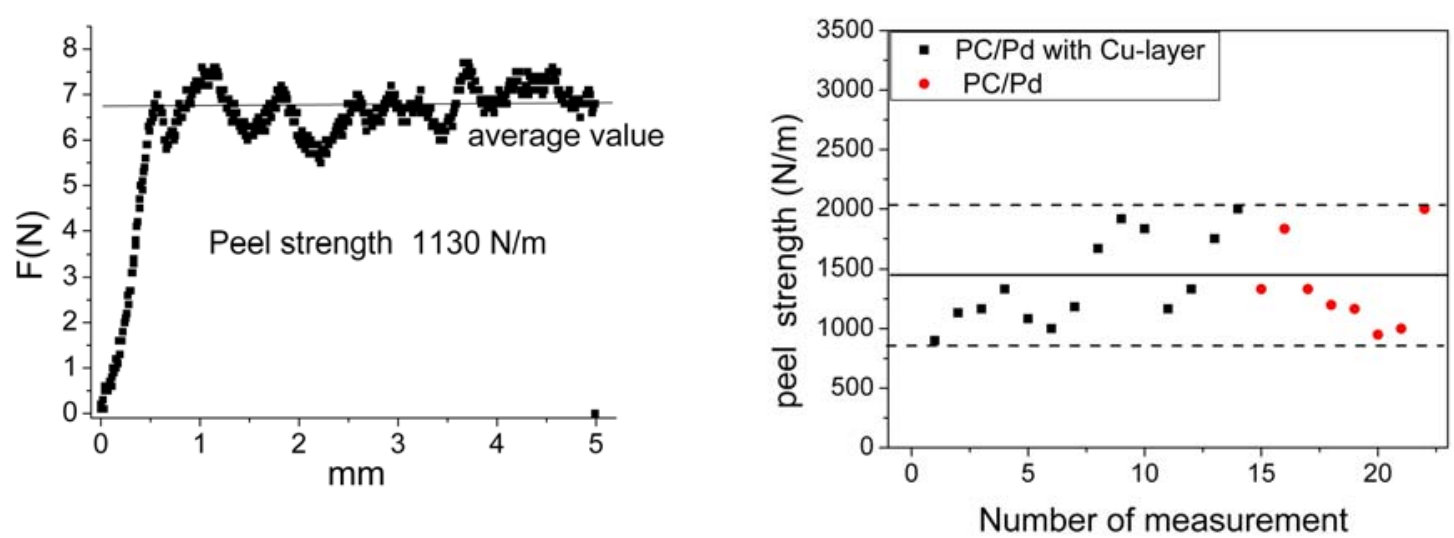

Figure 3.65: Peel-off measurement at the model system Pd/Nb/Pd//PC in cooperation with Prof. F. Faupel, University of Kiel. On the left a typical measurement is represented. The peel-off forces obtained in 22 individual measurements are summarized in the right field. The data strew about $40 \%$ around the average value of $1500 \mathrm{~N} / \mathrm{m}$.

The adhesion energy of Pd on PC can be calculated assuming that the peel strength work is equal to the work of separation. Then the work of separation $W_{S}$ can be expressed as

$$
W_{S}=\frac{F_{p} \cdot l_{p}}{A},
$$

where $F_{p}$ is the peel strength, $l_{p}$ is the way of peel force acting, $A$ is the detached area. For the average peel-off strength of $F=1500 \mathrm{~N} / \mathrm{m}, W_{S}$ is $113 \mathrm{~J} / \mathrm{m}^{2}$. This energy is much higher than 
the adhesion energy values of Pd on PC deduced by the other techniques applied in this work, and it includes mostly the energy dissipated for plastic deformation in the peel arm due to bending. Also the elastically stored energy in the peel arm and the energy dissipated within the substrate influences the peel force. Kendall has reported about the influence of the peel velocity on the peel force [K71]. The slower is the peel velocity the smaller is the peel force as well. Kendall has observed a difference of about $50 \%$ in the peel force by peeling with $0.3 \mathrm{~mm} / \mathrm{min}$ compared to $1.8 \mathrm{~mm} / \mathrm{min}$. The peel velocity applied in the measurements in the present work was $5 \mathrm{~mm} / \mathrm{min}$. This high velocity also might be a reason for the large peel strength values.

3.4.6 Buckling of $\mathrm{Pd} / \mathrm{Nb} / \mathrm{Pd}$ film on $\mathrm{PC}$ observed by $\mathrm{X}$-ray irradiation of the sample

Structural changes can be produced in polymers when these are irradiated in the solid state. The major effects observed refer to the formation of crosslinks and to the fracture of the main chain, depending on the particular polymer studied [M02]. This leads to the evolution of stress at the interface between a metal film and a polymer and therefore to adhesion weakening. This effect was observed by $\mathrm{X}$-Ray irradiation of $\mathrm{Pd} / \mathrm{Nb} / \mathrm{Pd}$ film on $\mathrm{PC}$ at HASYLAB. 
a)

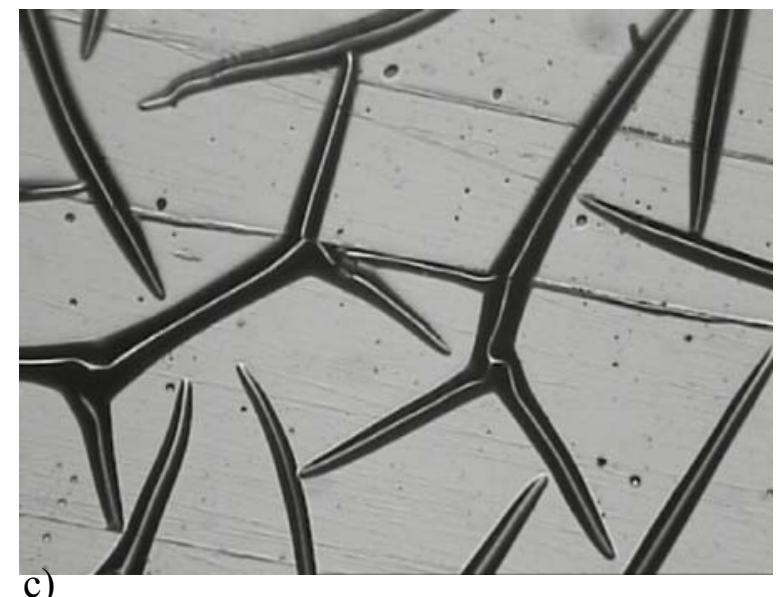

c)

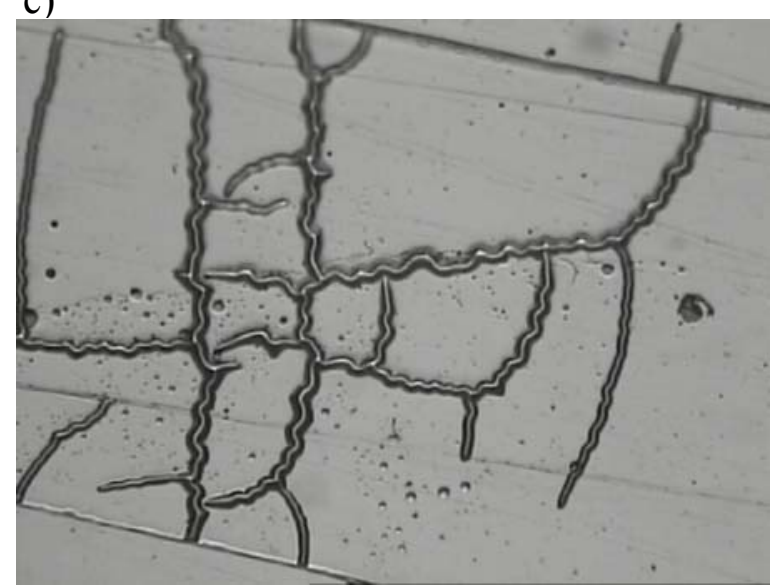

b)
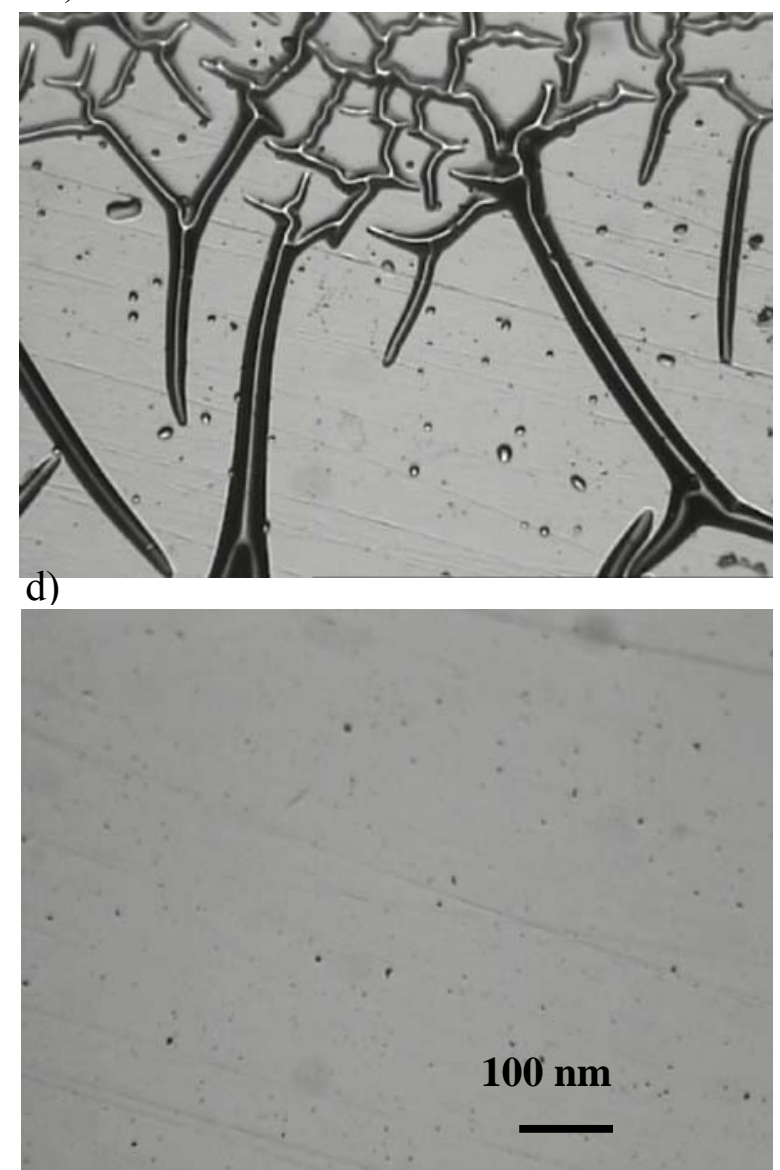

Figure 3.66: a) Large buckles in the region of the beam treated surface, b) at the edge of the beam, c) place close to beam d) $2 \mathbf{m m}$ millimetres apart from the beam-treated surface.

In Fig. 3.66 the appearance of the large buckles with $40-90 \mu \mathrm{m}$ width after 30 min beam treatment of the sample is shown. Buckles with a width about $16 \mu \mathrm{m}$ were found at the edge of the beam treated region. There again some millimetres from the beam treated region no buckles were found. The large buckles are a result of the compressive stress in the film.

Coffey et al. have reported [C98] about X-Ray radiation damage of PC in a scanning transmission X-Ray microscope. Nevertheless the energy of the synchrotron beam at DESY is much larger than that in a scanning transmission X-Ray microscope. Therefore a destroying influence of the beam on the polymer can be expected. For clarity, the effect of the beam treating is found in the breakage of bonds between neighbouring atoms, leading to the weakening of the adhesion energy and stress release in the metal film via buckling. Also the sample heating in the X-ray beam leads to stress increase at the metal-polymer interface and provides the buckle formation. 


\subsection{Hydrogen loading of niobium films on sapphire substrates}

In this chapter results on the electrochemical hydrogen loading of $\mathrm{Nb}$ films on sapphire will be presented. This system was used to investigate the stress development with respect to the delamination of $\mathrm{Nb}$ films, while their adhesion to sapphire is known to be very strong [EKR94, S97]. Also the bending behavior of the samples during hydrogen loading, emf measurements and observations linked to hydride formation will be described.

\subsubsection{Hydrogen loading of $800 \mathrm{~nm} \mathrm{Nb}$ films on sapphire}

The bonding of $\mathrm{Nb}$ to sapphire is a subject of experimental research since many years. These two materials bond strongly and the thermal expansion coefficients are similar, so that the residual stress at the interface after the sample preparation is minimized. The bonding depends on the orientation relationship in the cleavage plane of these two materials. The strongest bonding was observed by measurements of $\mathrm{Nb}(110)$ on (0001)-oriented sapphire substrates [EKR94]. The fracture energy of $\mathrm{Nb}(110)$ on (0001)-oriented sapphire substrates was measured to be $2107 \mathrm{~J} / \mathrm{m}^{2}$, while for the $\mathrm{Nb}(110)$ on (1-100)-orientated sapphire it is only $77 \mathrm{~J} / \mathrm{m}^{2}$.

$800 \mathrm{~nm} \mathrm{Nb}$ films on (0001)-orientated sapphire substrates with $0.2 \mathrm{~mm}$ thickness were sputtered using UHV cathode beam sputter technique. Even thicker $\mathrm{Nb}$ films of up to $4.7 \mu \mathrm{m}$ were prepared on similar substrates using the magnetron sputter technique (Chap. 2.1). Electrochemical hydrogen loading of these samples was performed with the focus on the mechanical stress, emf and buckling of these films. $0.2 \times 7 \times 30 \mathrm{~mm}^{3}$ dimensions of the sapphire substrates were chosen to be able to perform the bending measurements. The surface of $\mathrm{Nb}$ films was observed during hydrogen loading using a CCD camera.

The stress curve by the continuous electrochemical hydrogen loading of $\operatorname{Pd}(15 \mathrm{~nm}) / \mathrm{Nb}(800$ $\mathrm{nm}) / / \mathrm{Al}_{2} \mathrm{O}_{3}(0.2 \mathrm{~mm})$ with current density of $0.15 \mathrm{~mA} / \mathrm{cm}^{2}$ is shown in Fig 3.67. 
a)

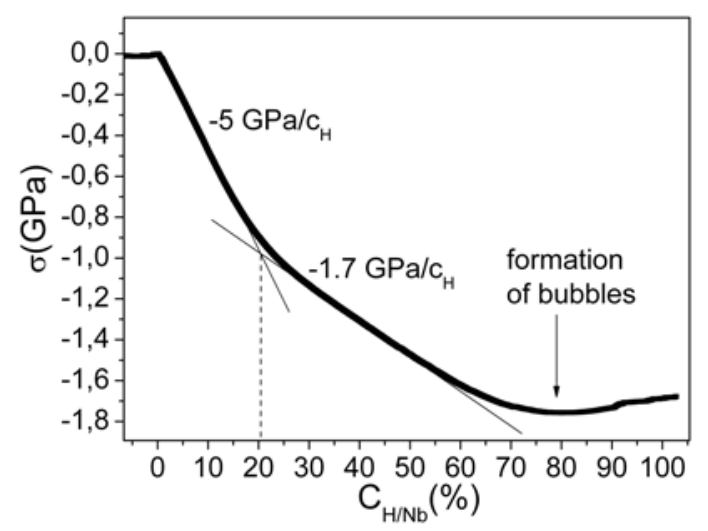

b)

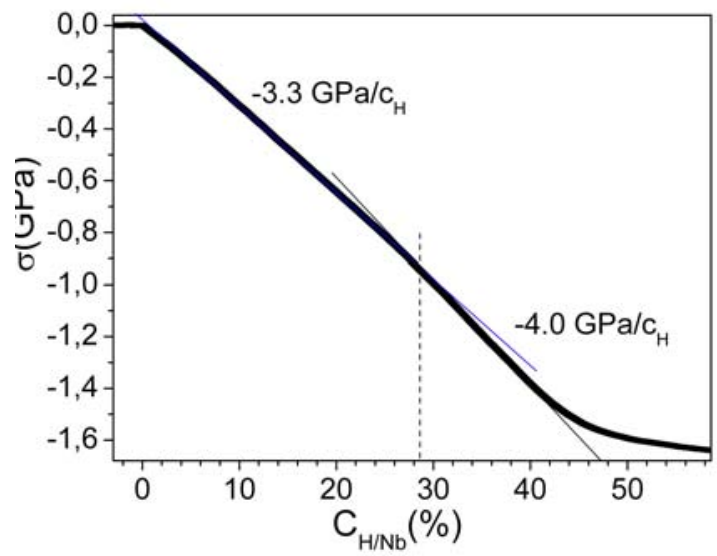

Figure 3.67: Stress curve during continuous hydrogen loading of $800 \mathrm{~nm}$ Nb-layer on (0001)-oriented sapphire substrate; a) first loading, b) second loading. In part a) the onset of the formation of hydrogen bubbles is marked, depicting that the hydrogen is not fully absorbed by the sample in this concentration range.

Two regions in the stress curve can be distinguished clearly. The first region presents the $\alpha$ phase region with a slope of $-5 \mathrm{GPa} / \mathrm{c}_{\mathrm{H}}$. In the two-phase region the slope is decreased and amounts $-1.7 \mathrm{GPa} / \mathrm{c}_{\mathrm{H}}$. At about $80 \% \mathrm{H} / \mathrm{Nb}$ the bubbles formation of hydrogen at the film surface is observed. The second loading shows an increase of the slope with maximum value of $-4 \mathrm{GPa} / \mathrm{c}_{\mathrm{H}}$. This maximum value relates to a rather low $\mathrm{H}$-content $\mathrm{c}_{\mathrm{H} / \mathrm{Nb}}$.

Fig. 3.68 shows emf ${ }^{*}$ of the sample (see Chap. 3.1.4, p.51) measured during the continuous hydrogen loading. An increase of the chemical potential in the $\beta$-phase region of $\mathrm{Nb}-\mathrm{H}$ system is determined. The emf was further increased as can be seen in Fig. 3.68 up to $70 \% \mathrm{H} / \mathrm{Nb}$ by about $70 \mathrm{mV}$. This can be explained by Pd-hydride formation. The formation of Pd-hydride was observed for $190 \mathrm{~nm} \mathrm{Nb}$ film with $30 \mathrm{~nm}$ Pd coping layer at about $100 \% \mathrm{H} / \mathrm{Nb}$ [Lau98]. The increase of the electrochemical potential by formation of $\mathrm{Pd}-\mathrm{H}$ by about $110 \mathrm{mV}$ was measured by Yang et al. [Y96] (reference: saturated calomel electrode). 


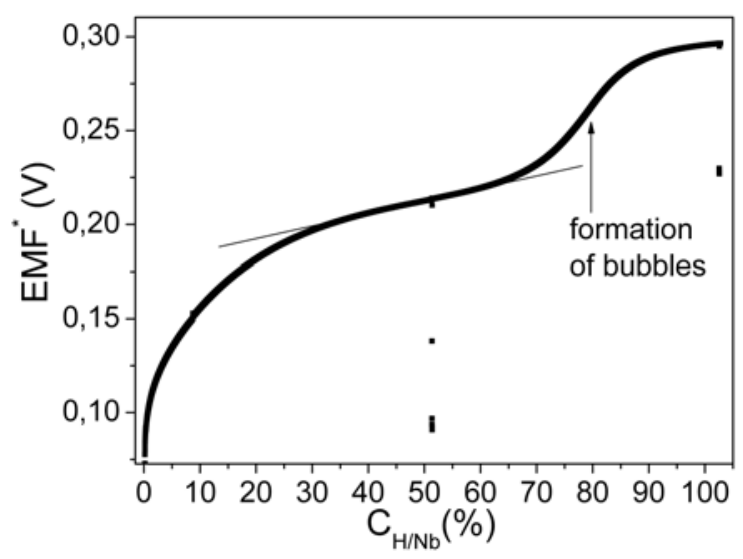

Figure 3.68: Emf of the $800 \mathrm{~nm}$ Nb-layer with $30 \mathrm{~nm}$ Pd top layer on (0001)-oriented sapphire substrate during hydrogen loading.

Fig. 3.69 presents the stress relaxation of the $800 \mathrm{~nm} \mathrm{Nb}$ on sapphire after the first loading.
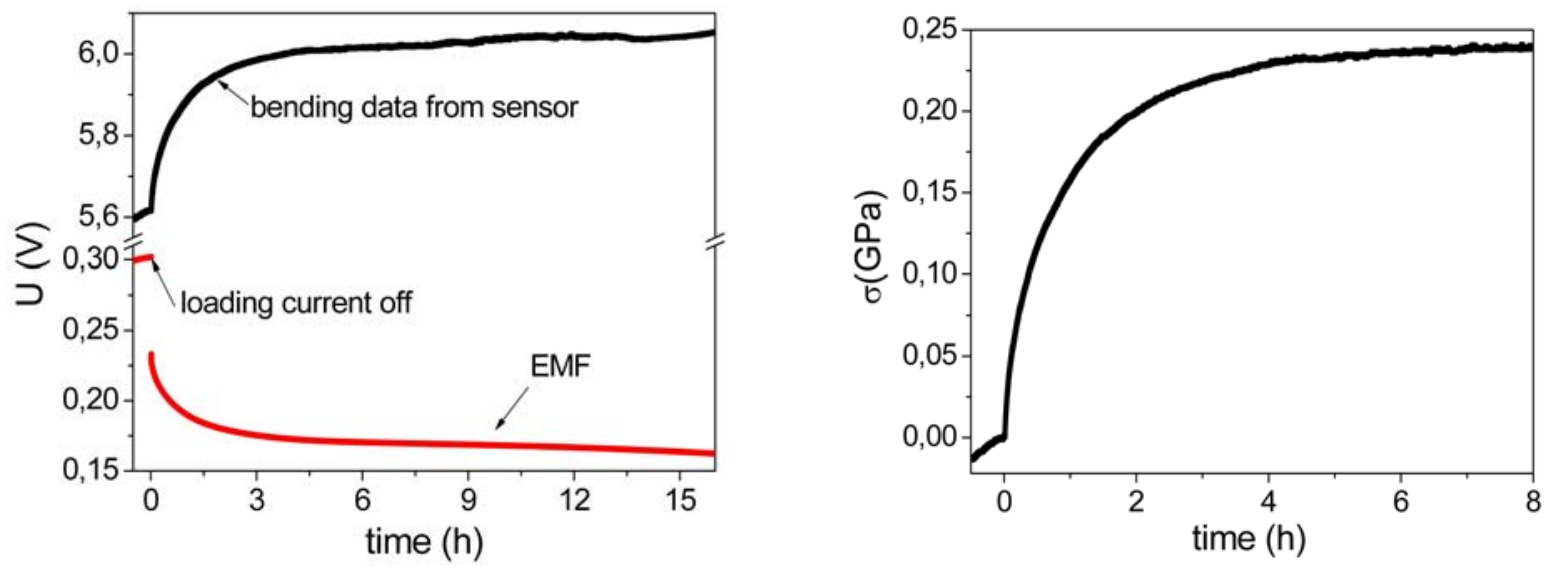

Figure 3.69: Stress relaxation after the first hydrogen loading of $800 \mathrm{~nm} \mathrm{Nb}$ on sapphire. a) Emf related to the data of the bending measurement, b) stress change by relaxation.

The maximal mechanical stress reached during the first loading sequence was about $-1.75 \mathrm{GPa}$. Within 8 hours the stress relaxes by about $0.23 \mathrm{GPa}$ (about $13 \%$ of the maximal value). $90 \%$ of the stress relaxation occurs within the first 4 hours. Thereby the stress relaxation is caused by the hydrogen loss. Taking into account the upper limit of hydrogen content in this sample of $80 \%$ and hydrogen loss proportional to the stress relaxation, the hydrogen loss was about $10 \% \mathrm{H} / \mathrm{Nb}$ Buckles formation was not observed in these studies. As can be seen in Fig 3.70 (b), small black regions appears after hydrogen loading in the light microscopically image. These are film extrusions caused by dust particles on the sapphire substrate prior to sputtering (see Chap. 3.5.5) 
a)

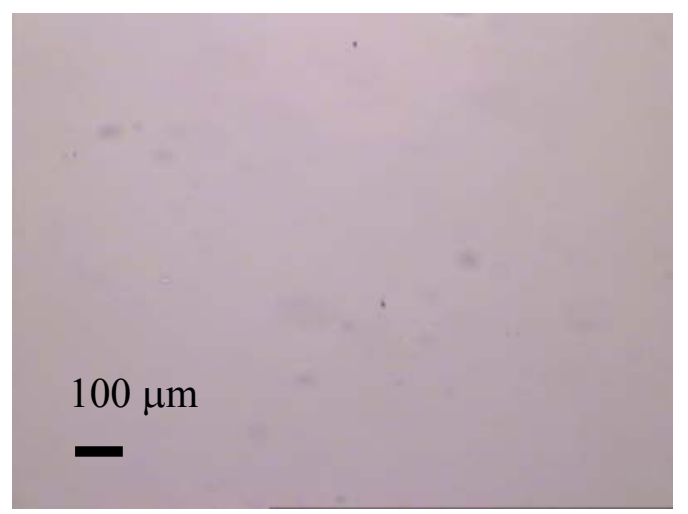

b)

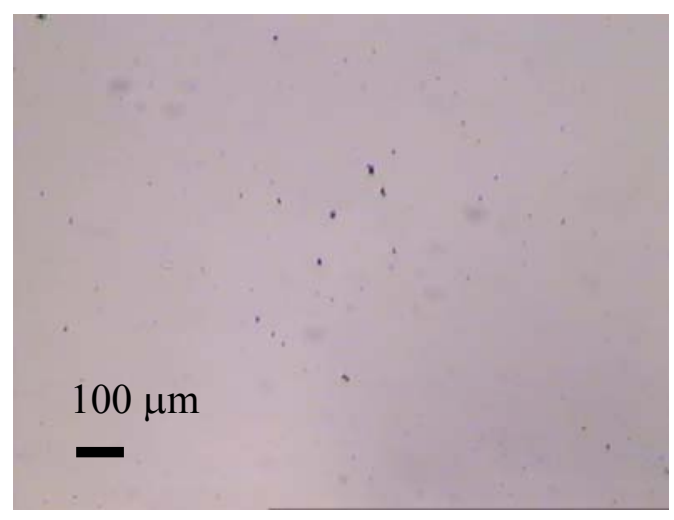

Figure 3.70: Light microscopy images of $800 \mathrm{~nm}$ Nb-layer on (0001)-oriented sapphire substrate, a) before hydrogen loading, b) after hydrogen loading.

Due to the fact that no buckle formation was observed during hydrogen loading of $800 \mathrm{~nm} \mathrm{Nb}$ layer on sapphire substrate, thicker films were prepared. This was done to force the film towards detachment, because the elastic energy that can be stored in the film is proportional to the film thickness (see Eqs. 1.27, 1.31).

\subsubsection{Hydrogen loading of $1.95 \mu \mathrm{m}$ and $2.5 \mu \mathrm{m} \mathrm{Nb}$ film on sapphire}

In order to exceed the delamination limit, $1.95 \mu \mathrm{m} \mathrm{Nb}$ were loaded with hydrogen until $100 \%$ $\mathrm{H} / \mathrm{Nb}$ using a current density of $0.15 \mathrm{~mA} / \mathrm{cm}^{2}$. The resulting stress curve for stepwise hydrogen loading is plotted in Fig. 3.71. A bending point at $8 \% \mathrm{H} / \mathrm{Nb}$ can be seen. The $\alpha-(\alpha, \beta)$ phase transition was found to set in at $6.7 \% \mathrm{H} / \mathrm{Nb}$, as the investigation of the related emf-curve in Fig. 3.74 shows.

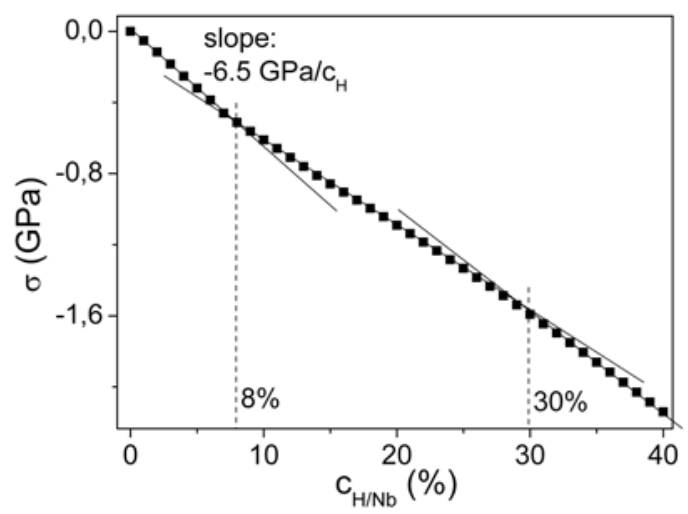

Figure 3.71: Stress curve for stepwise electrochemical hydrogen loading of $1.95 \mu \mathrm{m} \mathrm{Nb}$ film on sapphire. 
Fig. 3.72 presents the bending data recorded from the inductive sensor during the stepwise electrochemical hydrogen loading.
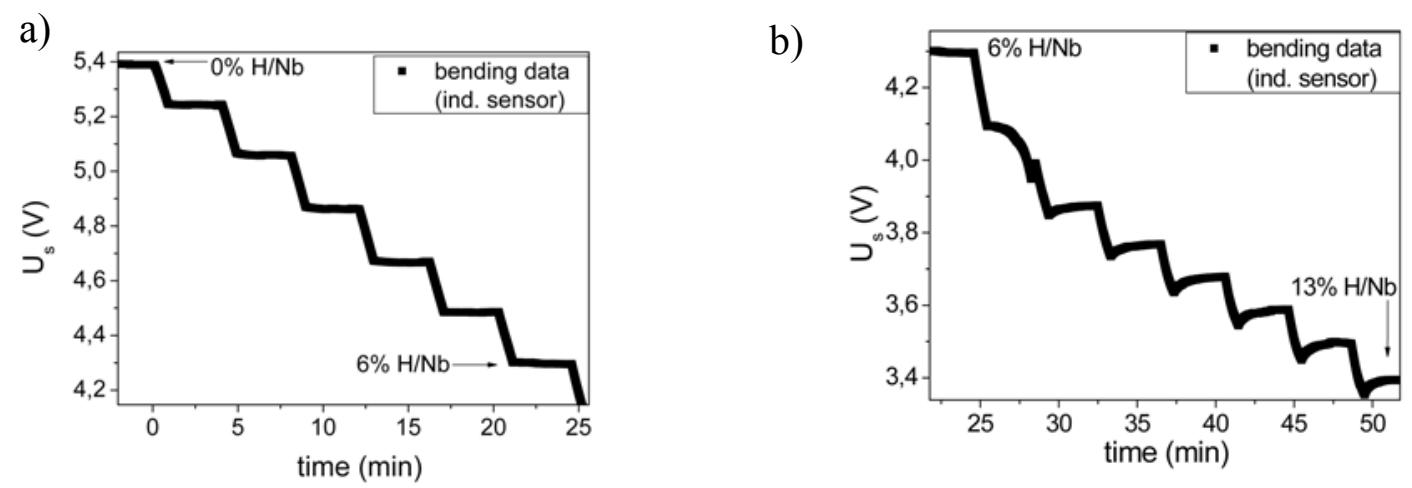

c)

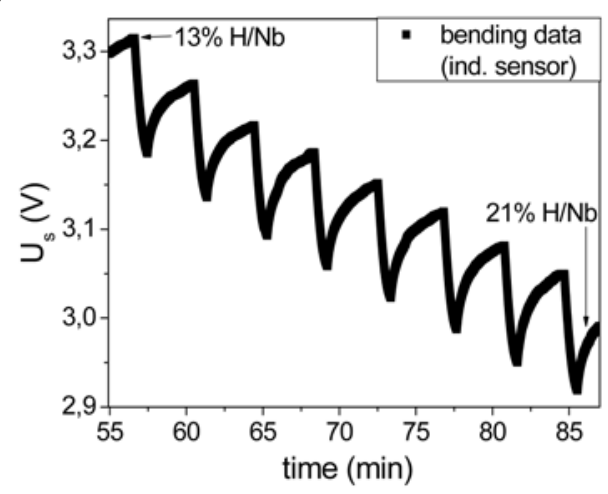

Figure 3.72: Bending data during hydrogen loading of 1.95 $\mu \mathrm{m} \mathrm{Nb}$ film on sapphire, a ) 0-6\% $\mathrm{H} / \mathrm{Nb}$, b)6-13\% $\mathrm{H} / \mathrm{Nb}$, c) $13-21 \% \mathrm{H} / \mathrm{Nb}$.

During $1-7 \% \mathrm{H} / \mathrm{Nb}$-loading the sample does not relax between the loading steps. In the further hydrogen loading sequences a decreasing bending of the sample between the loading steps was observed, showing relaxation.

In the second loading cycle of this sample no relaxation process during hydrogen loading was observed, as can be seen in Fig. 3.73.

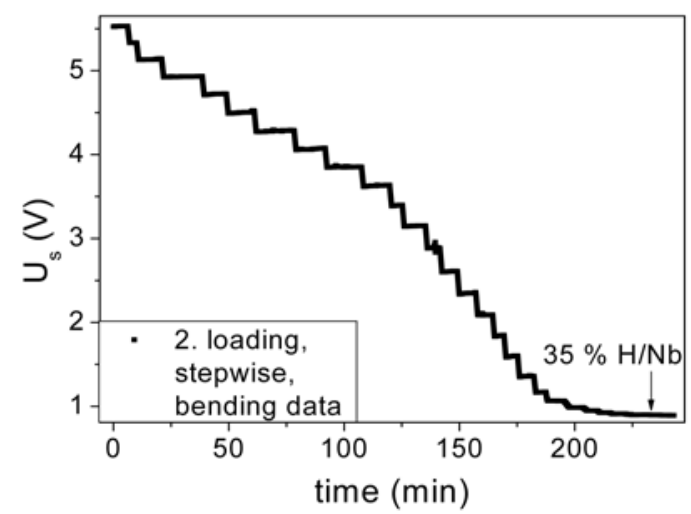

Figure 3.73: Bending data recorded during the second hydrogen loading of $1.95 \mu \mathrm{m} \mathrm{Nb}$ layer on 0.2 mm sapphire substrate. 
The rate of the increase of compressive stress is reduced at about $35 \% \mathrm{H} / \mathrm{Nb}$ and in principle no further stress increase was observed.

The emf values during stepwise electrochemical hydrogen loading are shown in Fig. 3.74.

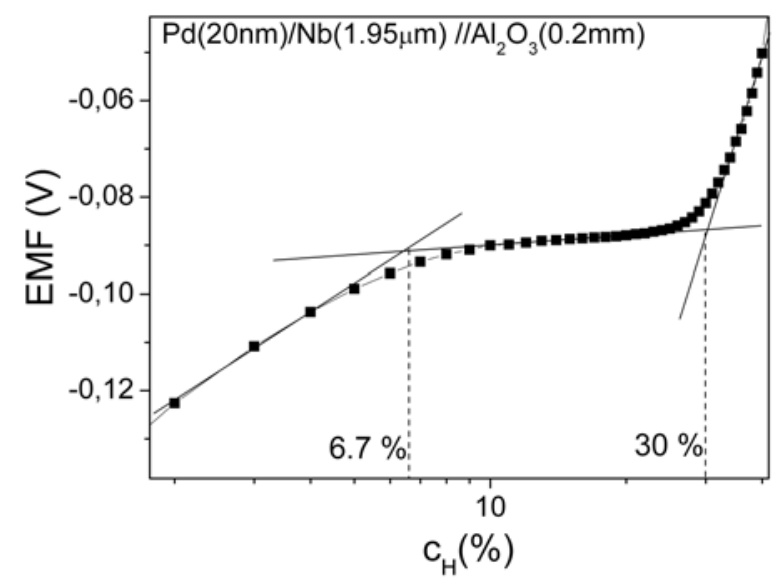

Figure 3.74: Emf of $1.95 \mu \mathrm{m} \mathrm{Nb}$ film on sapphire during first loading sequence on a logarithmical scale.

Emf curve for the first hydrogen loading shows a two-phase plateau region. From Fig. 3.74 the phase boundaries in the virgin sample are determined as $6.7 \% \mathrm{H} / \mathrm{Nb}$ for the $\alpha-(\alpha, \beta)$ transition and $30 \% \mathrm{H} / \mathrm{Nb}$ for the $(\alpha, \beta)-\beta$ transition.

Fig. 3.75 shows the bending data during unloading of the sample after the first loading sequence. After 4.5 hours unloading was nearly complete and sample-bending deviated by about $5 \%$ only from its start value before hydrogen loading.

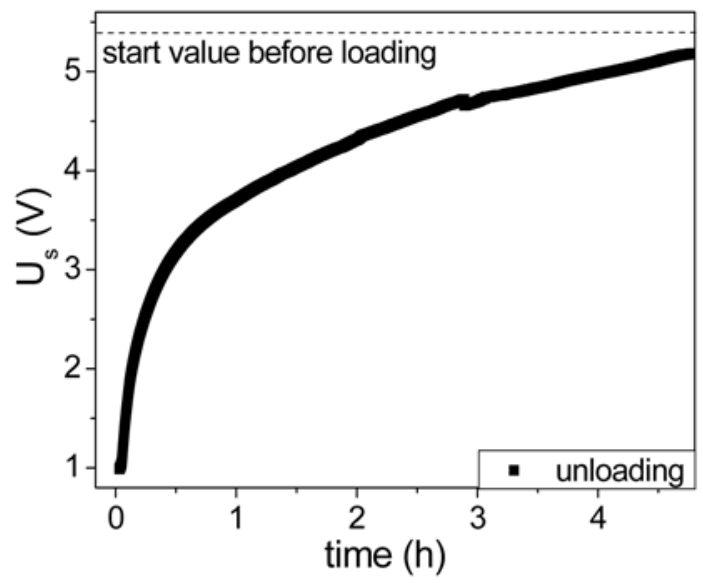

Figure 3.75: Bending data during hydrogen unloading process. 95\% of stress after hydrogen loading is relaxed after 4.5 hours unloading. 
To summarise shortly, the inflection points of the stress curve (Fig. 3.71) are correlated with the phase transitions in $\mathrm{H}-\mathrm{Nb}$, which were determined by emf measurements shown in Fig. 3.74. The relaxation after loading steps is stronger at the hydrogen concentrations in the two phase region and there was no relaxation during the second loading, as it is shown in Fig. 3.73. Stress measurement is very helpful for the investigation of the hydrogen unloading process to estimate the degree of unloading.

It has to be pointed out that no buckling was observed during the hydrogen loadings of 1.95 $\mu \mathrm{m} \mathrm{Nb}$ on sapphire.

Next, hydrogen loading of $2.5 \mu \mathrm{m}$ Nb-layer on sapphire applying a current density of $0.15 \mathrm{~mA} / \mathrm{cm}^{2}$ was performed. Fig. 3.76 shows the bending data for the hydrogen loading of this sample.

a)

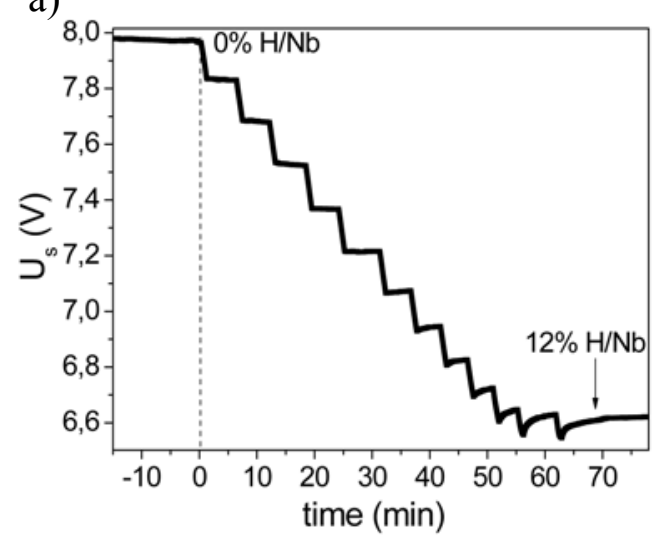

b)

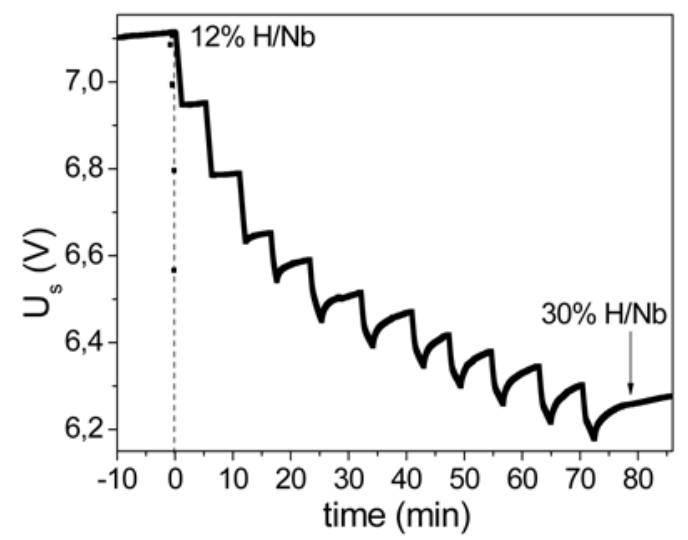

Figure 3.76: Bending data for the hydrogen loading of $2.5 \mu \mathrm{m} \mathrm{Nb}$-layer on $0.2 \mathrm{~mm}$ sapphire substrate; a) 0-12\% loading, b) 12-30 \% loading.

The loading sequence of this sample was interrupted at $12 \% \mathrm{H} / \mathrm{Nb}$ for 24 hours to perform REM investigations of this sample. The total emf curve is shown in Fig. 3.77.

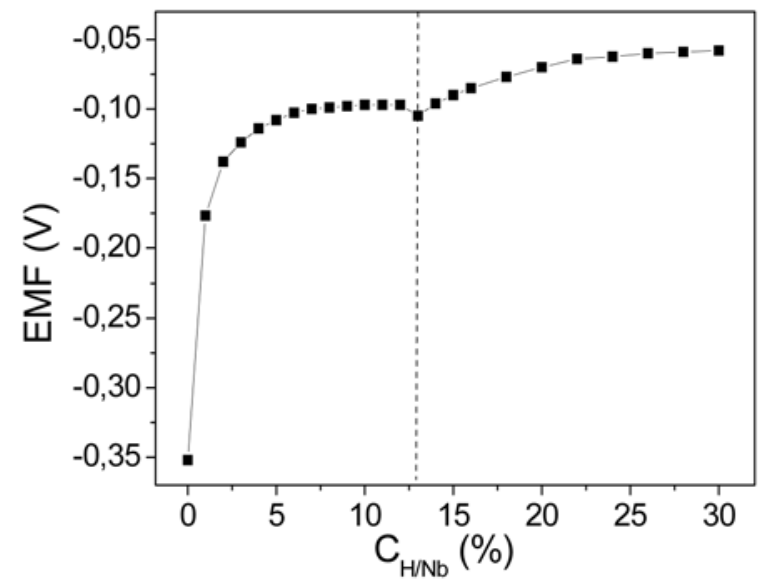

Figure 3.77: Emf curve during the stepwise electrochemical hydrogen loading of $2.5 \mu \mathrm{m} \mathrm{Nb}$-layer on $0.2 \mathrm{~mm}$ sapphire substrate. The loading sequence was interrupted at $12 \% \mathrm{H} / \mathrm{Nb}$ for one day. 
The emf value after the interruption of hydrogen loading visible in the emf curve is comparable with the emf value of $6 \% \mathrm{H} / \mathrm{Nb}$. Therefore hydrogen loss amounting to 3-6\% $\mathrm{H} / \mathrm{Nb}$ is assumed, which might be the reason why the bending data depicted in Fig. 3.76(b) do not show relaxation in the first steps, while the stress relaxation during the further loading steps are typical for the two phase region.

Again, no buckles are formed during the loading sequence of this sample.

Afterwards hydrogen unloading of this sample was performed to test the reversibility of the optical surface morphology changes (see appendix A.1, Fig. A.2). The unloading was carried out via changing the polarity of the sample and the reference electrode and applying a current density of $\mathrm{j}=0.35 \mathrm{~mA} / \mathrm{cm}^{2}$. The bending data during the unloading process are shown in Fig. 3.78.

a)

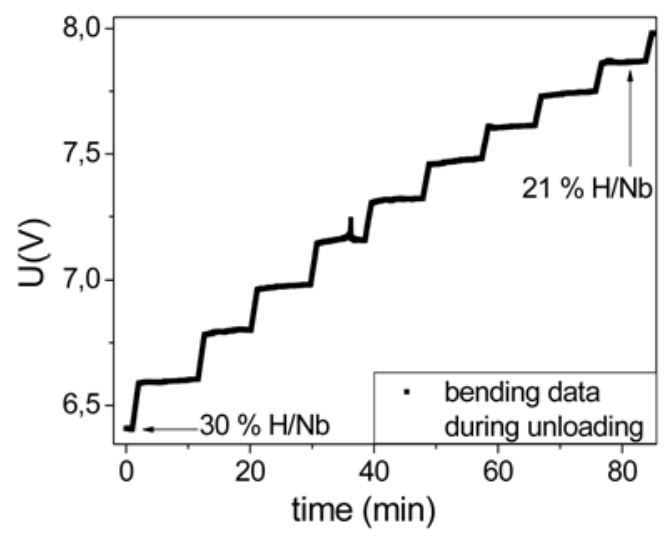

c)

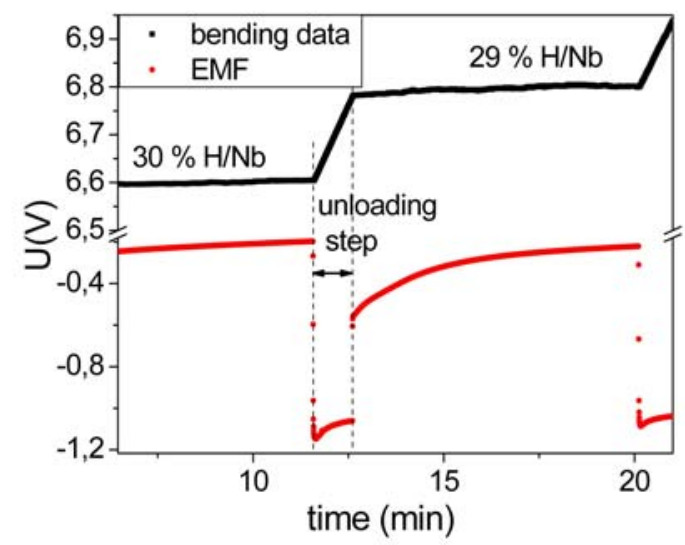

b)

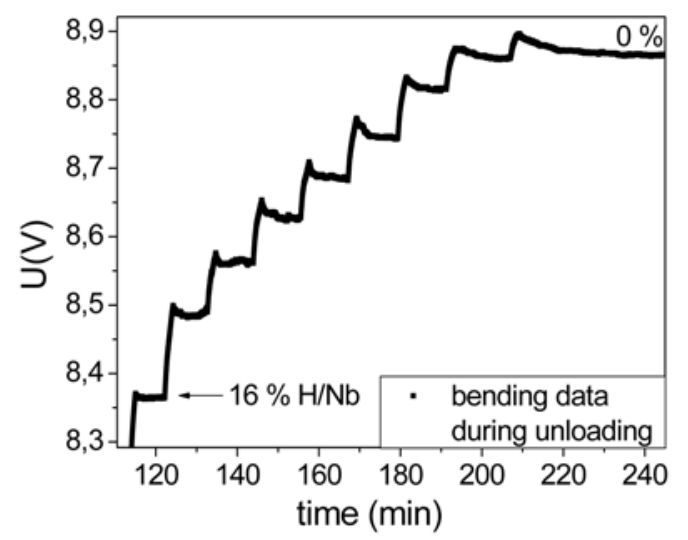

d)

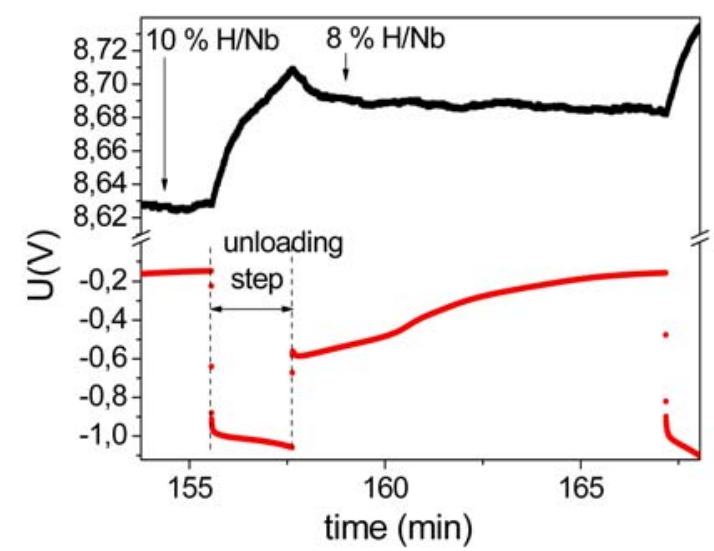

Figure 3.78: Bending data recorded during the unloading process of $2.5 \mu \mathrm{m}$ Nb-layer on $0.2 \mathrm{~mm}$ sapphire substrate; a) unloading $30-21 \% \mathrm{H} / \mathrm{Nb}$, b) $16-0 \% \mathrm{H} / \mathrm{Nb}$, c) $30-29 \% \mathrm{H} / \mathrm{Nb}$, d) $10-8 \% \mathrm{H} / \mathrm{Nb}$.

The optical monitoring of the unloading process has shown no reversibility in the appearance of the surface roughness, which was detected by using the light microscopy during hydrogen loading. It is questionable if the unloading process is fully reversible and if the estimated 
hydrogen concentrations during unloading are correct. The concentrations are given assuming the reversibility of electrochemical hydrogen loading. While no relaxation process was detected during the unloading from 30 to $21 \% \mathrm{H} / \mathrm{Nb}$, the tendency of relaxation increased with the further unloading procedure. The relaxation took place within 1-4 min. It is assumed to be faster if more hydrogen atoms are still in the metal film and to take more time if the amount of hydrogen in the sample is small because of larger driving forces (difference in the chemical potential of $\mathrm{H}$ in the film and in the electrolyte) in the first case.

\subsubsection{Hydrogen loading of $4 \mu \mathrm{m}$ and $4.75 \mu \mathrm{m} \mathrm{Nb}$ on sapphire}

$4 \mu \mathrm{m}$ thick Nb-layers on sapphire were loaded with hydrogen: One sample was loaded continuously, another sample was loaded stepwise. The current density for these loading sequences was $0.35 \mathrm{~mA} / \mathrm{cm}^{2}$. The stress curves for these loading sequences are shown in Fig. 3.79. The slopes of these stress curves were unexpectedly small. Further measurements are needed to understand this unexpectedly small stress.

a)

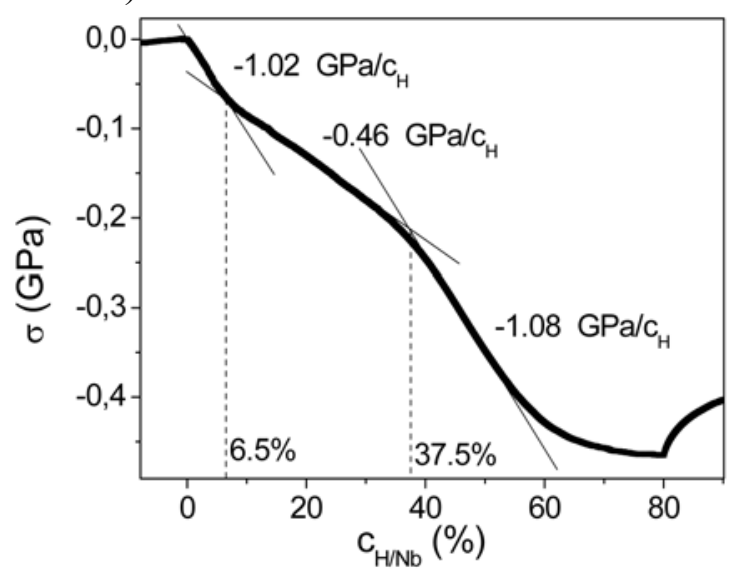

b)

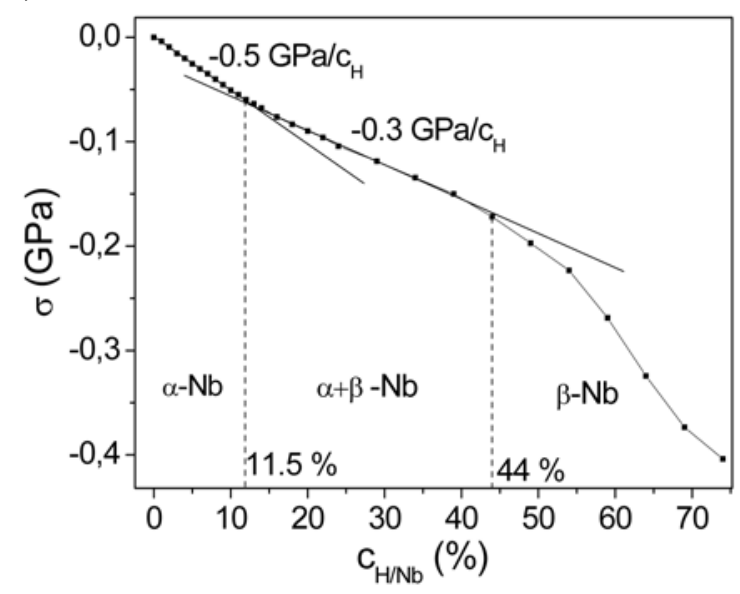

Figure 3.79: Stress curves for hydrogen loading of $4 \mu \mathrm{m}$ Nb-layer on $0.2 \mathrm{~mm}$ sapphire substrate; a) continuous loading, b) stepwise loading.

Two bending points are visible in the stress curves. The deflection points during stepwise loading thereby occur at higher concentrations than during continuous loading. This effect can be 
explained by the formation of hydrides (which is most probably a surface hydride) at lower concentrations than by stepwise loading, because the continuous loading was faster.

Images of the related surface morphology changes at comparable H-concentrations for stepwise and continuous loading are compared in Fig. 3.80.

a) $31 \% \mathrm{H} / \mathrm{Nb}$, continuous loading

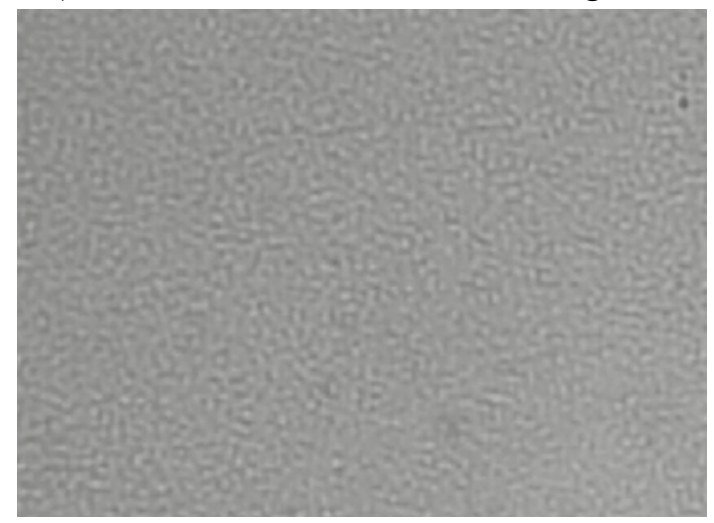

b) $39 \% \mathrm{H} / \mathrm{Nb}$, stepwise loading

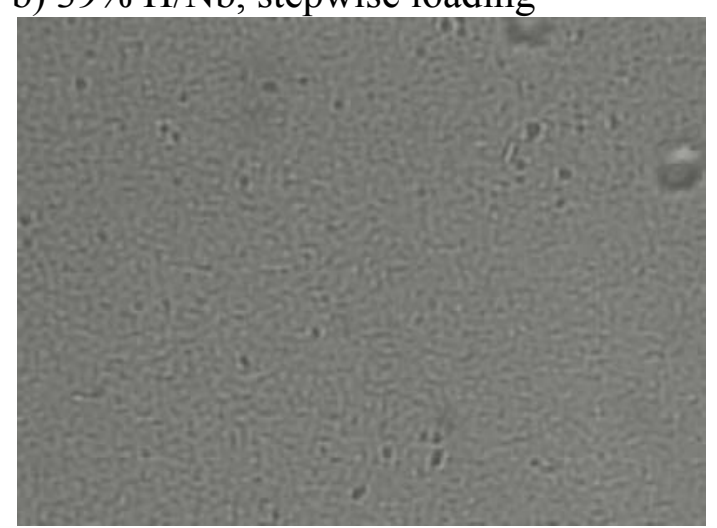

Figure 3.80: Light microscopically images of $4 \mu \mathrm{m}$ Nb-layer on $0.2 \mathrm{~mm}$ sapphire substrate during hydrogen loading; a) surface at $31 \% \mathrm{H} / \mathrm{Nb}$ during continuous hydrogen loading, b) Surface at $39 \%$ $\mathrm{H} / \mathrm{Nb}$ by stepwise hydrogen loading.

The change of the surface morphology which is assumed to be caused by hydride formation occurs at the same time when the stress curve deflects. At $78 \% \mathrm{H} / \mathrm{Nb}$ during continuous loading crack formation in the film was observed, while in the stepwise loaded film cracks appeared at $76 \% \mathrm{H} / \mathrm{Nb}$. The film was destructed in the buckle region during buckle formation. This delaminated area is shown in Fig. 3.81.

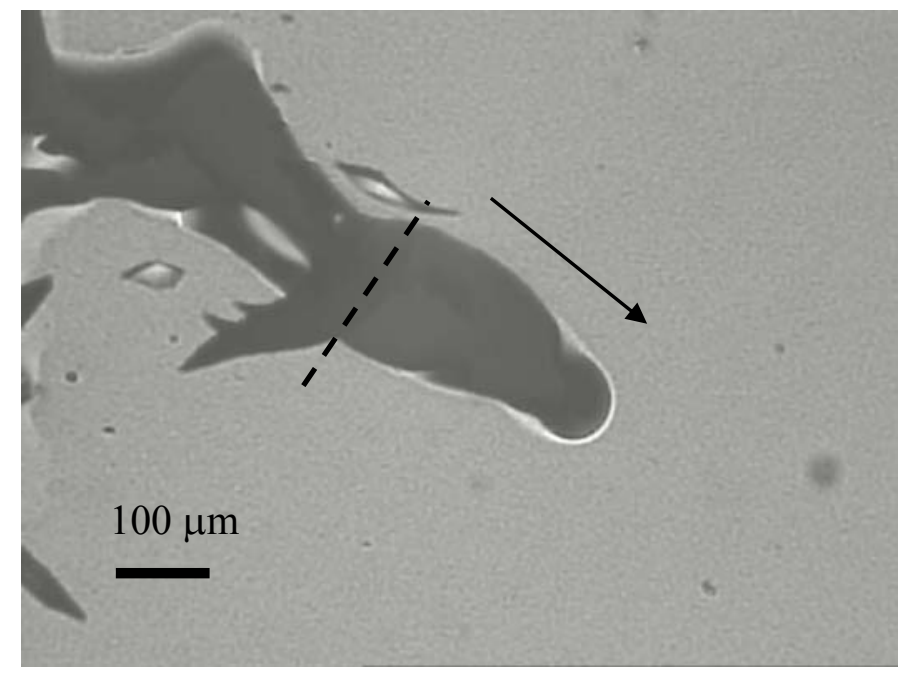

Figure 3.81: Delamination with destruction of $4 \mu \mathrm{m}$ Nb-layer on $0.2 \mathrm{~mm}$ sapphire substrate during hydrogen loading. The arrow shows the direction of buckle propagation.

The buckle in Fig. 3.81 has a width of about $120 \mu \mathrm{m}$, measured along the dotted line. The film residues from the destructed film in the buckle region are also visible in the figure. Applying Eq. 
(1.23) and taking the critical stress value from Fig. 3.79 (a), a theoretical buckle width of $119 \mu \mathrm{m}$ from Euler mode was calculated. This is in a very good agreement with the measured buckle width.

It can also be pointed out that the completion of the $\beta$-phase formation occurs at about $40 \% \mathrm{H} / \mathrm{Nb}$, while this value for bulk niobium is $72 \% \mathrm{H} / \mathrm{Nb}$. The reason for this is a high mechanical stress in the metal films. This effect was investigated by Dornheim [Dor02].

The maximal thickness of $\mathrm{Nb}$ films investigated in this work was $4.75 \mu \mathrm{m}$. The bending data during electrochemical loading of such a film are shown in Fig. 3.82.

a)

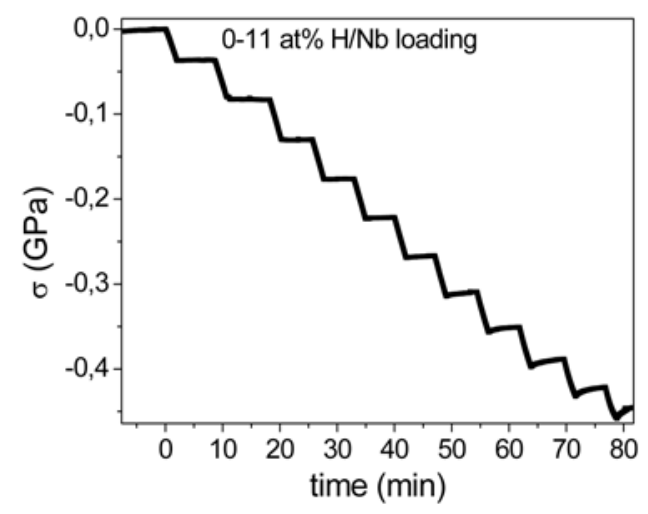

b)

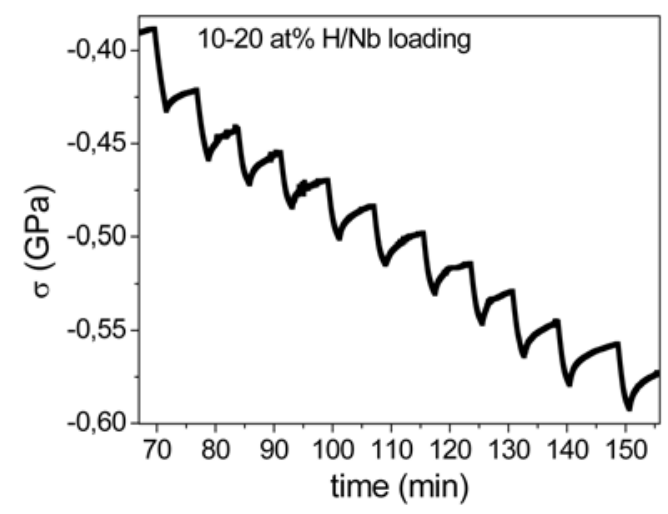

Figure 3.82: Bending data during hydrogen loading of $4.75 \mu \mathrm{m} \mathrm{Nb}$ film on sapphire, a) $0-11 \% \mathrm{H} / \mathrm{Nb}, \mathrm{b}$ ) $10-20 \% \mathrm{H} / \mathrm{Nb}$.

In Fig. 3.82 again an increase of the stress relaxation at the hydrogen concentrations in the two phase region is visible. The upper boundary of the two phase region was found to be $43 \% \mathrm{H} / \mathrm{Nb}$, as shown in Fig. 3.83 .

a)

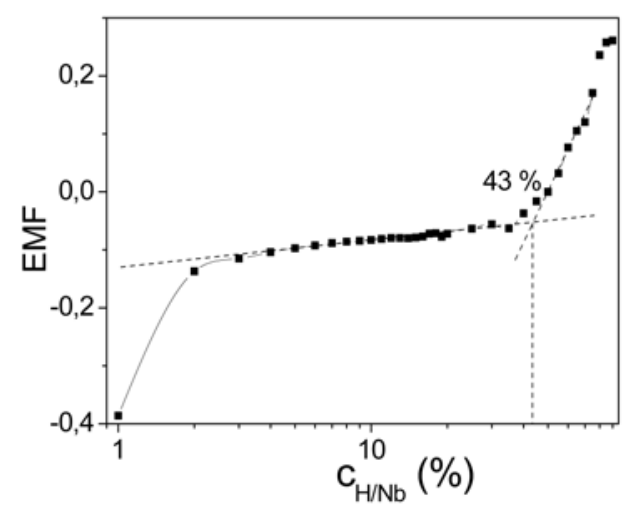

b)

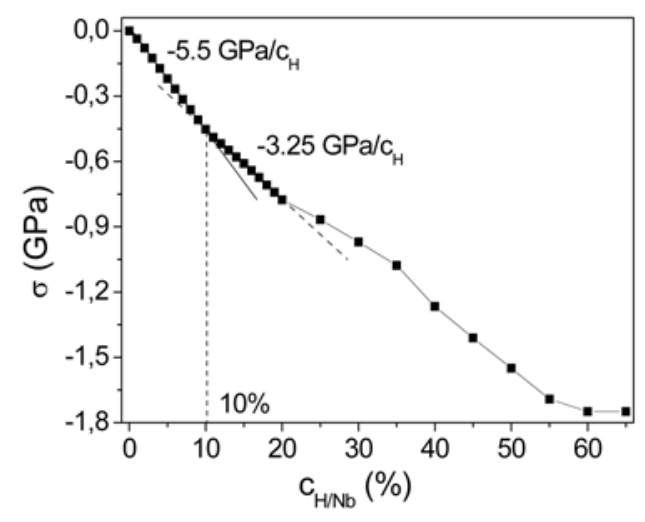

Figure 3.83: Stepwise hydrogen loading of $4.75 \mu \mathrm{m} \mathrm{Nb}$ film on sapphire. a) Emf curve on logarithmical scale, b) stress curve. 
An inflexion point can be seen at $10 \% \mathrm{H} / \mathrm{Nb}$ in the stress curve (Fig. 3.83b), which correlates with optical changes of the surface morphology at $11 \% \mathrm{H} / \mathrm{Nb}$ (see appendix A.1, Fig. A.4). The delamination of this film appeared at $63 \% \mathrm{H} / \mathrm{Nb}$. The delaminated regions are shown in Fig. 3.84 .

a)

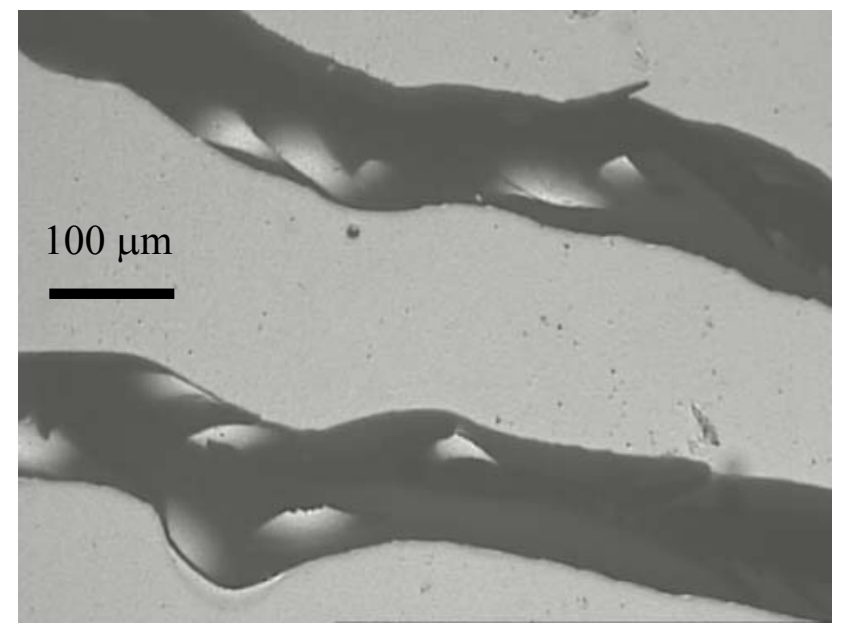

c)

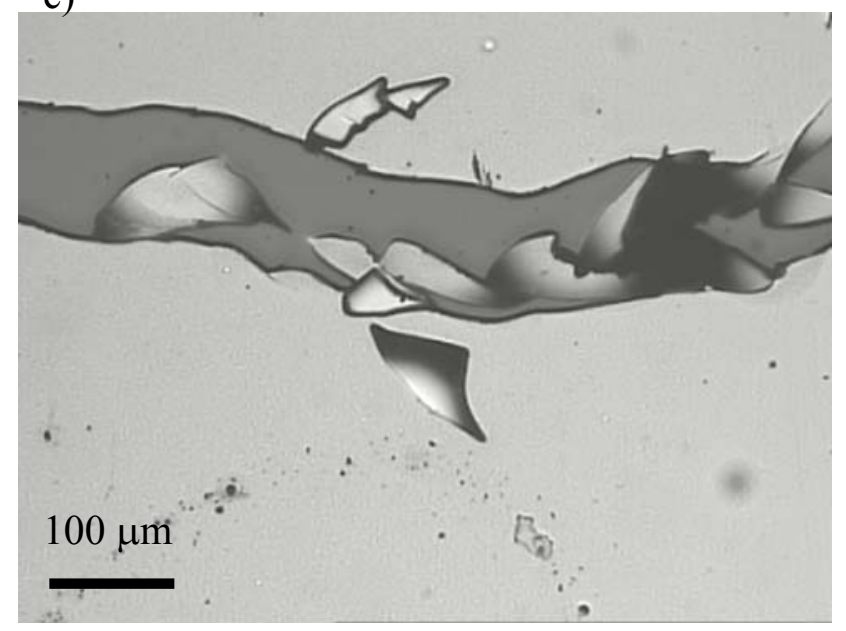

b)

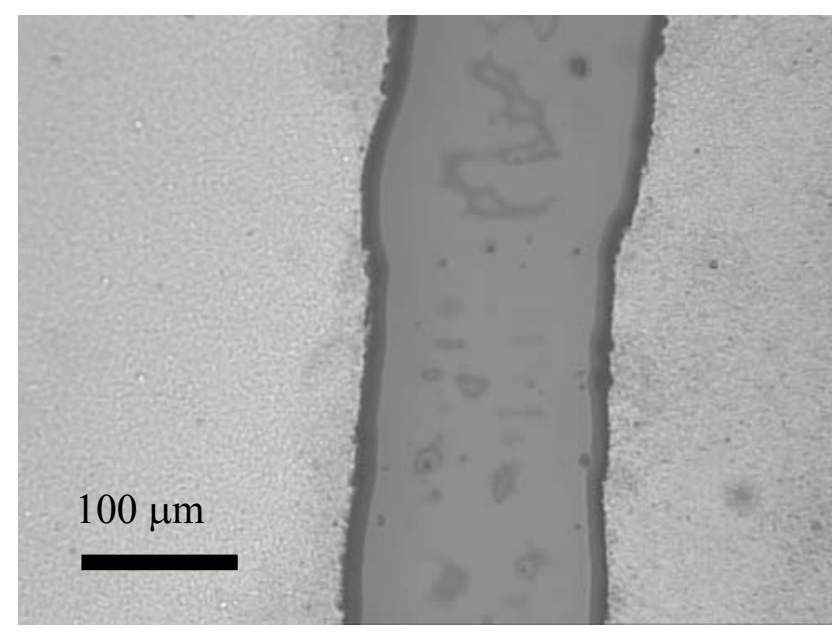

d)

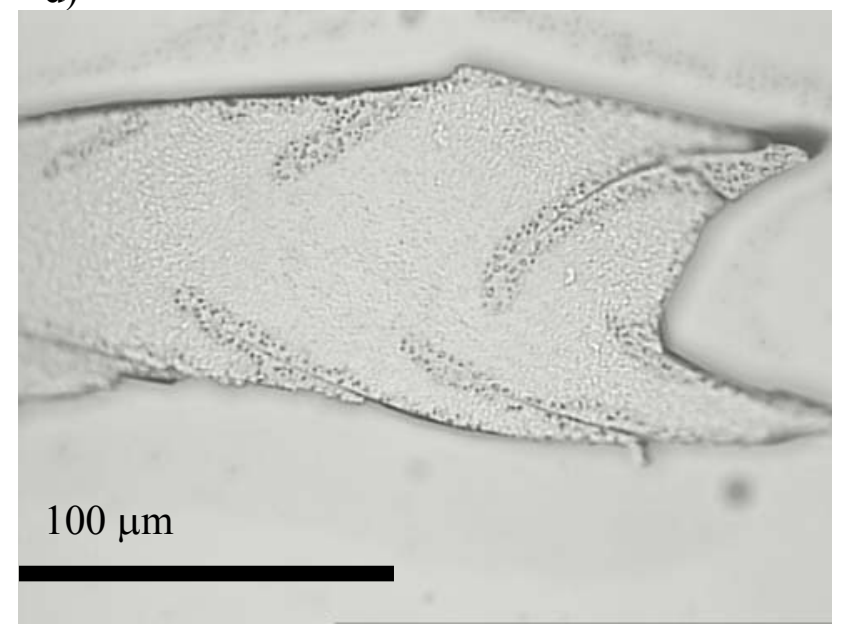

Figure 3.84: Buckle formation of $4.7 \mu \mathrm{m} N \mathrm{~N}$ film on sapphire during hydrogen loading with film cracking. a), b), c) regions of buckles; d) residues of $\mathrm{Nb}$ film after destruction of the film in the delaminated region.

\subsubsection{Adhesion energy between $\mathrm{Nb}$ and sapphire}

The adhesion energy of $\mathrm{Nb}$ films on sapphire can be estimated by using Eq. 1.34 and the critical concentrations of niobium layers with $4 \mu \mathrm{m}$ and $4.7 \mu \mathrm{m}$ film thicknesses on $0.2 \mathrm{~mm}$ sapphire (0001). The critical concentrations are shown in Tab. 3.9. 
Table 3.9: critical hydrogen concentrations for buckling of $\mathrm{Nb}$ on sapphire

\begin{tabular}{|l|l|l|}
\hline Thickness of Nb-layer, $(\mathrm{nm})$ & 4000 & 4700 \\
\hline $\mathrm{c}_{\mathrm{H} / \mathrm{Nb}, \mathrm{crit}},(\%)$ & $78 \pm 2$ & $63 \pm 2$ \\
\hline
\end{tabular}

The plot of $c_{\text {crit }}^{2}$ versus inverse film thickness is shown in Fig. 3.85.

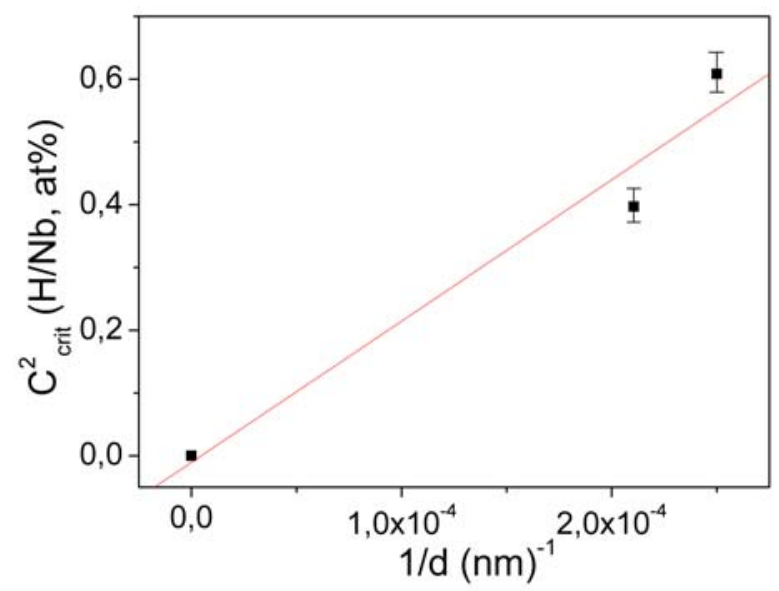

Figure 3.85: $c_{c r i t}^{2}$ as a function of the inverse film thickness for $4 \mu \mathrm{m}$ and $4.7 \mu \mathrm{m} \mathrm{Nb}$ films on sapphire with a linear fit through the zero point.

As the third point in the plot the zero point was used. This assumption was made because it is assumed that the critical hydrogen concentration for buckling for films with infinite thickness should be very small. The adhesion energy calculation following (1.34) then gives an adhesion energy of $\gamma=450 \mathrm{~J} / \mathrm{m}^{2}$. The condition expressed in Eq. 1.42, which determinates if the critical stress for buckling provides the information about adhesion energy was checked using the buckle width from Fig. 3.81 and the theoretical adhesion energy $\gamma_{\mathrm{Nb} / \mathrm{saphire}}=10 \mathrm{~J} / \mathrm{m}^{2}$ (see [B00]. The condition is fulfilled. This large adhesion value of $\mathrm{Nb}$ on sapphire was expected, since it was not possible to detach $\mathrm{Nb}$ films with thicknesses below $4 \mu \mathrm{m}$ from their substrates.

Elssner et al. [EKR94] have found a large dependency of the crystallographic orientations of sapphire and $\mathrm{Nb}$ on the fracture energy (see Fig. 3.86). They found that the adhesion energy of the $\mathrm{Nb}$ /sapphire interface depends on the interface orientation of both materials. The energy of plastic deformations of $\mathrm{Nb}$-film by delamination also depends on the relation of the glide planes and glide directions to the interface and on the direction of the crack propagation. Fracture energy values for the different niobium-sapphire interface orientations estimated by Elssner are shown in Fig. 3.86. 


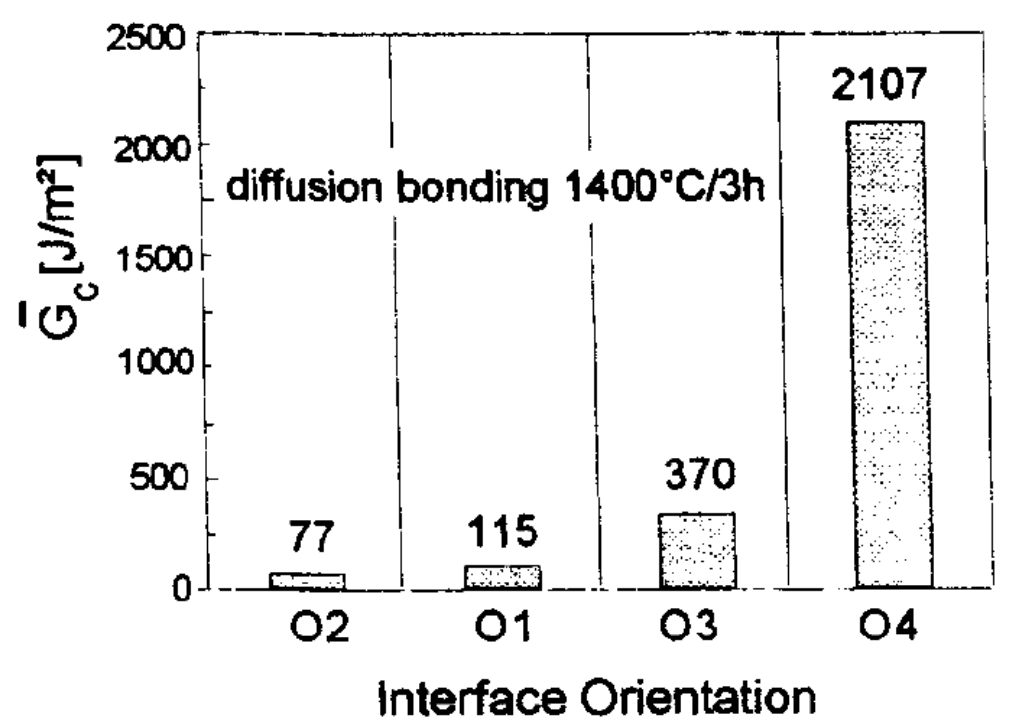

Figure 3.86: Fracture energy as a function of the crystallographic orientations 01 to 04 of niobium/sapphire interfaces (from [EKR94]). The samples in the work of Elssner were prepared by diffusion bonding carried out at $1400^{\circ} \mathrm{C}$ for 3 hours under a compressive stress of $10 \mathrm{MPa}$.

The $\mathrm{O} 4$ orientation is for the $\mathrm{Nb}$ (110) [001]//Sapphire (0001) [1-100]. For the $\mathrm{Nb}$ films investigated in this work the texture has been determined and it is shown in Fig. 3.87 in a (110) pole figure for a $4 \mu \mathrm{m} \mathrm{Nb}$ film on sapphire (0001).

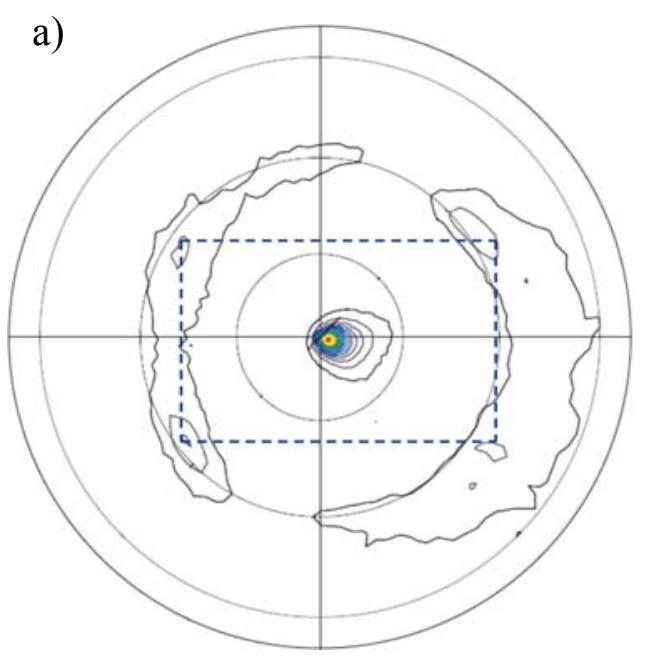

b)

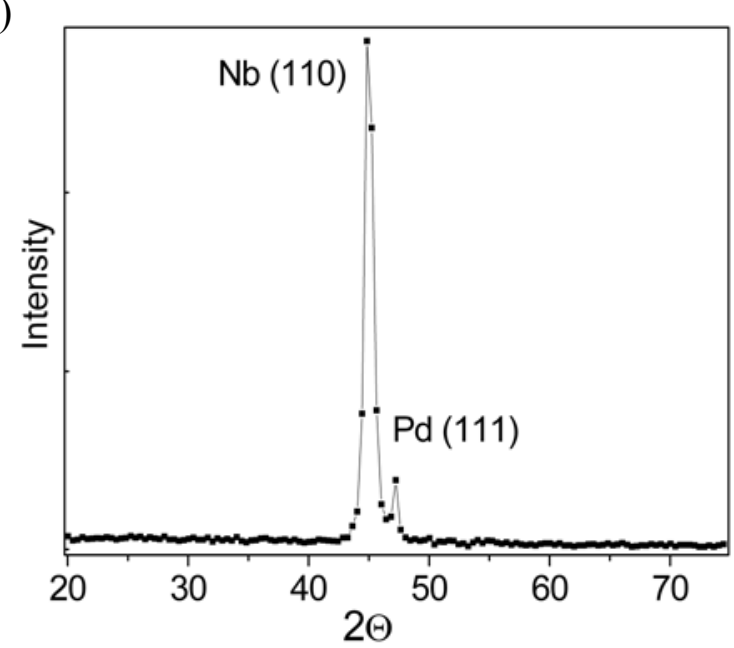

Figure 3.87: $X$-ray texture investigation of a $\mathrm{Nb}$ film on sapphire. a) $\mathrm{Nb}(110)$ pole figure of a $4 \mu \mathrm{m} \mathrm{Nb}$ film on sapphire; dotted lines shows a domain of the Nb lattice; b) XRD overview scan of a $4 \mu \mathrm{m} \mathrm{Nb}$ film on sapphire.

The texture measurement shows a strong $\mathrm{Nb}(110)$-reflex, the other reflexes of this group are very weak but they can be indicated as well. 


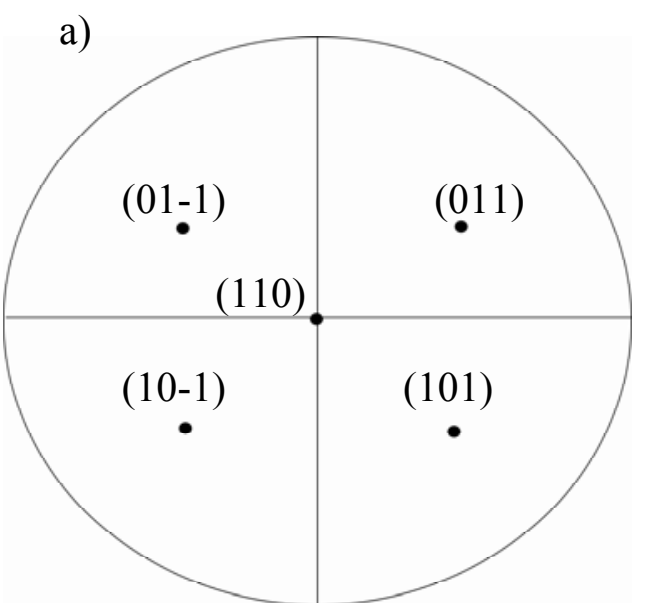

b)

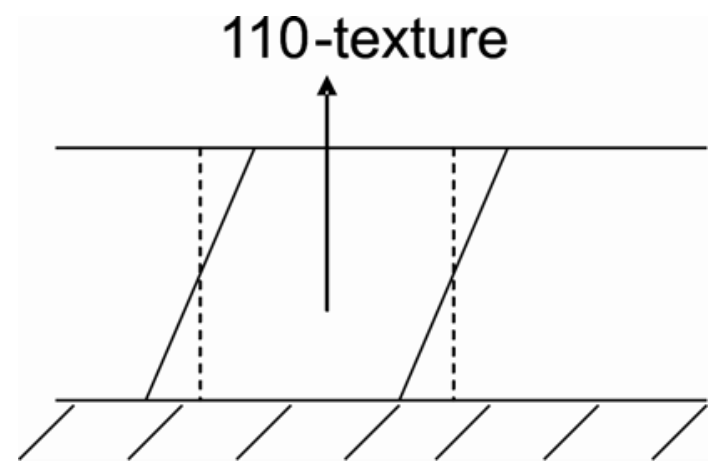

Figure 3.88: Analyses of the X-ray texture measurement. a) Schematical $N b$ (110) pole figure, b) grains of $\mathrm{Nb}$ film are tilted out of the substrate normal.

The texture measurement verified that the crystallographic interface orientation of the $\mathrm{Nb}$ sapphire samples investigated in this work is similar to the interface orientation O4 in Fig. 3.86, which exhibits large fracture energy of the niobium-sapphire interface of $2107 \mathrm{~J} / \mathrm{m}^{2}$. The tilting of the grain orientations also explains the smaller adhesion energy measured in this work.

Because of the destruction of the film during delamination (see Fig. 3.84) the dissipated work of plastic deformation in the $\mathrm{Nb}$ film is large. Therefore the energy calculated from the data of Fig. 3.85 reflects not exclusively adhesion energy, but includes the fracture energy of niobium on sapphire for the given interface crystallographic orientation, as well.

To summarise this part of the work it can be concluded that it was possible to determine the fracture energy of $\mathrm{Nb}$ /sapphire interface, which is very large in comparison to the adhesion energy values of polymer-metal interfaces. However, for the systems with strong bonding films with large thickness are needed. The detachment of such films is linked with large plastic deformations. Thus, it was not possible to detach the $\mathrm{Nb}$ films in soft manner.

\subsubsection{Influence of stress in $\mathrm{Nb}$ films on hydride formation}

Hydride formation observed using optical microscope occurs predominantly around the defects in the film. This can be seen in Fig. 3.89, where the black points are the film defects in form of film extrusions, as can be seen in Fig. 3.90. Lower stress around the defects leads to higher hydrogen concentration in these areas due to the Gorsky effect. 
a)

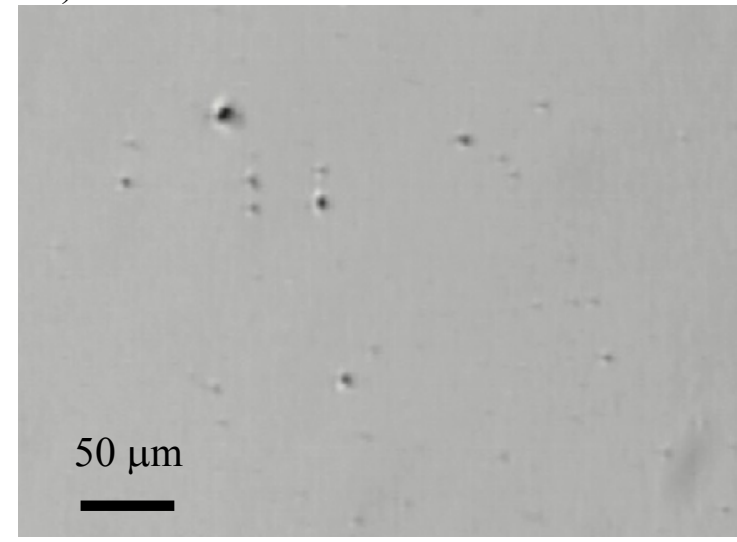

b)

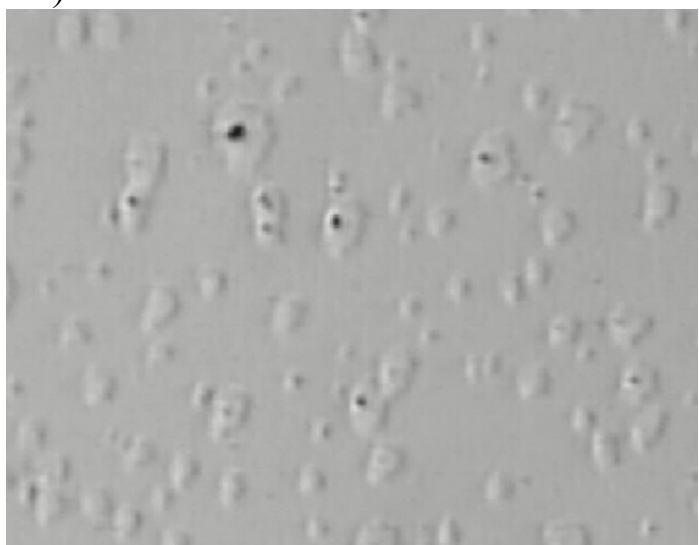

Figure 3.89: Optical observation of niobium hydride formation in $P d(20 \mathrm{~nm}) / \mathrm{Nb}(2.5 \mu \mathrm{m})$ on $0.2 \mathrm{~mm}$ sapphire substrate. a) $10 \% \mathrm{H} / \mathrm{Nb}, \mathrm{b}) 11 \% \mathrm{H} / \mathrm{Nb}$

The size of the black areas in Fig. 3.89 is about 1-16 $\mu \mathrm{m}$. These black regions in the optical image occur in the centers of the hydride precipitates. Magnifications of the black areas with the Scanning Electron Microscopy (SEM) are shown in Fig. 3.90.
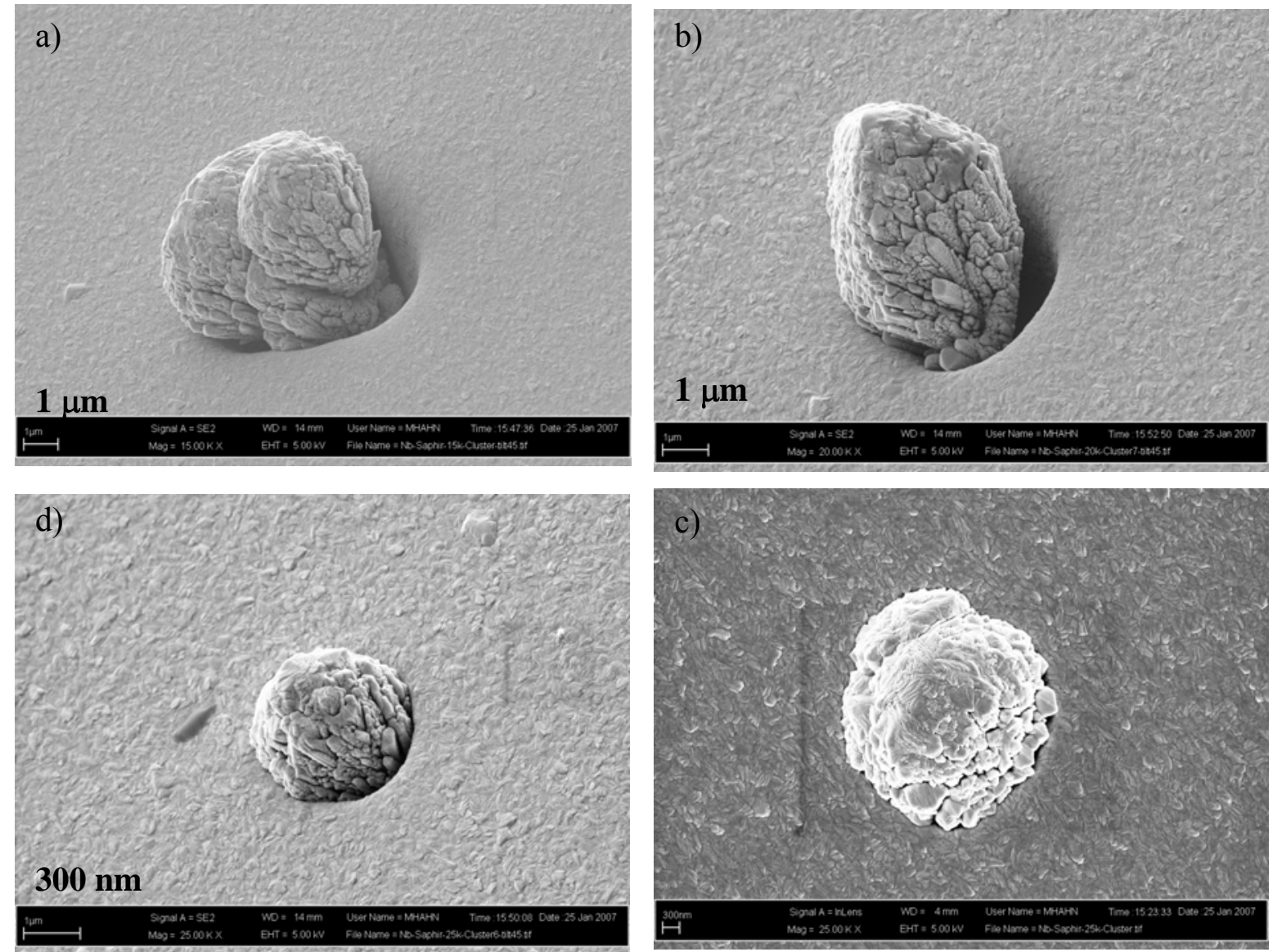

Figure 3.90: SEM images of the $\operatorname{Pd}(20 \mathrm{~nm}) / \mathrm{Nb}(2.5 \mu \mathrm{m})$ on $0.2 \mathrm{~mm}$ sapphire sample; a), b), c) sample is tilted at $45^{\circ}, d$ ) top view. 
To conclude, the black areas in the optical images of the surface after film preparation are the film extrusions depicted in Fig. 3.90. The surface of the extrusions is rough, explaining the dark colored appearance of the extrusions in the optical images. It is supposed that the formation of the extrusions was caused by dust particles present on the sapphire substrate prior to sputtering. The bonding of the film sputtered on the dust particles is much lower and therefore these parts were pressed out of the film probably after the sputtering process by film absorption of a small amount of hydrogen in air. The lower film stress around the extrusion causes larger hydrogen concentrations in these areas (Gorsky effect) and supports hydride formation in these areas, shown in Fig. 3.89. 


\section{Discussion}

Hydrogen loading of the investigated $\mathrm{Pd} / \mathrm{Nb} / \mathrm{Pd} / / \mathrm{PC}$ model system is discussed, focussing on buckling and the evaluation of the critical parameters for buckling.

\subsection{Hydrogen loading of model films on polycarbonate}

Electrochemical hydrogen loading of the model system $\mathrm{Pd} / \mathrm{Nb} / \mathrm{Pd}$ on $\mathrm{PC}$ with respect to buckling was presented in Chap. 3.1. The large hydrogen solubility of the $\mathrm{Nb}$ layer allows the introduction of high stresses in this system. Thin Pd layers prevent oxidizing of the $\mathrm{Nb}$ layer and act as a catalyst for hydrogen loading. Thus, a $\mathrm{Nb}$ film with $\mathrm{Pd}$ coping layer can absorb a large amount of hydrogen producing high compressive stress if this system is adhering to the substrate. The influence of film- and substrate-thicknesses on such measurements was investigated. It was found that thinner films delaminate at higher hydrogen concentrations as it is shown in Chap. 3.1.2. The critical hydrogen concentrations for $\mathrm{Pd} / \mathrm{Nb} / \mathrm{Pd}$ films on $1 \mathrm{~mm} P C$ with $50 \mathrm{~nm}, 100 \mathrm{~nm}$, $200 \mathrm{~nm} \mathrm{Nb}$-layers and $10 \mathrm{~nm} \mathrm{Pd}$ layers were $17 \% \mathrm{H} / \mathrm{Nb}, 11 \% \mathrm{H} / \mathrm{Nb}$ and $7 \% \mathrm{H} / \mathrm{Nb}$, respectively. Such behaviour is expected when the elastic energy stored in the film is proportional to the film thickness, as calculated in Eq. (1.27). The critical hydrogen concentrations are independent of the $\alpha-\beta$ phase transition, which was found to occur at $20 \% \mathrm{H} / \mathrm{Nb}$ for $200 \mathrm{~nm} \mathrm{Nb}$ film on sapphire [Lau98]. The size of hydride-precipitates does not correspond to the size of buckles, which is about one order of magnitude larger [N06]. A linear relation between the critical hydrogen concentrations to the inverse film thicknesses as it is shown in Eq. 1.34 was confirmed experimentally. Further it was found that the thickness of the substrate affects $\mathrm{c}_{\text {crit. }}$. For thinner substrates $\mathrm{c}_{\text {crit }}$ is higher. A reason for that was found in the straining of thin polymer substrates due to the stress in the Nb-films during hydrogen loading. This effect changes the stress and, therefore, the critical hydrogen concentration.

A critical parameter has been proposed for adhesion energy determination, which is the critical stress for buckling. The critical stress for buckling can be used if the stress measurement parameters are chosen properly. This was confirmed in Tab. 3.6 and can be achieved when a high accuracy of the geometry of the substrates is kept with exactly the same geometry of all films and substrates which are used for the adhesion energy determination. The clamping of samples in the measuring setup should be optimized for each substrate thickness (the clamping part of the sample should be not to short). It should be pointed out that the critical hydrogen concentrations are easier to measure than stress. The stress measurement equipment is not necessary in this case 
and the sample geometry is not important. Therefore, the measurement of the critical hydrogen concentrations for buckling is recommended in this work to determine adhesion energies of films on mechanically rigid substrates. Films on rigid substrates give reproducible results, which can be used for the calculations of adhesion energy, because no elastic energy dissipates by substrate stretching, observed during hydrogen loading of films on $20 \mu \mathrm{m}$ PC substrates in Chap.3.1.3. The polycarbonate substrates with a thickness of more than $1 \mathrm{~mm}$ were found to act as rigid substrates, see Chap. 3.1.3.

For data analysis, deviation of $2 \%$ from the linear dependency of the stress curves and the resulting critical hydrogen concentrations for buckling gave results that were in accordance with the values obtained by optical monitoring of the sample surfaces. The critical concentrations taking for more than $2 \%$ deviation from the linear dependency of the stress curves result in larger adhesion energy values. However, the related absolute stress values were not reproducible in our case as it is shown in Fig. 3.17.

The cracking interface by the detachment of the metal from the polymer substrate was shown in chap. 3.2.4 to be the interface between the metal and the polymer. Crack formation in the metal is very improbable because of the much stronger metal/metal bonding than the polymer/metal bonding. If the crack propagates inside of the polymer, the metal-polymer interface should have no effect and adhesion values independent of the metal would be expected, which was not observed for the films with different metal layers on PC.

Two types of electrochemical hydrogen loading were applied in this work: stepwise hydrogen loading and continuous loading. Both types have advantages and disadvantages for the investigation of buckling and adhesion energy determination. The continuous loading allows better evaluation of the stress development and a more reliable determination of the phase transitions and the buckling processes. Also the total time of the hydrogen loading sequence is shorter. Thus a long contact time between the sample and the electrolyte is avoided, which can weaken the interface by the dissolution of water, and which can reduce hydrogen loss from the metal film (see Chap. 3.1.4). Grundmeier [GS05] described the mechanisms of water adsorption at polymer/metal interfaces leading to de-adhesion, and he proposed experimental methods for investigation of this phenomenon. The disadvantages are inhomogeneous hydride formation (surface hydride) and incomplete hydrogen absorption by applying high loading current. Stepwise hydrogen loading can be useful if a detailed investigation of the sample after each loading step is required. It has to be pointed out that hydrogen loading of $4 \mu \mathrm{m} \mathrm{Nb}$ film on sapphire shows the hydride formation at higher hydrogen concentration during stepwise hydrogen loading compared to continuous loading as it is shown in Fig. 3.79. The reason for that 
is a longer time for the stress relaxation after each loading step and homogenous hydride formation in the whole volume of the $\mathrm{Nb}$ film by stepwise loading. The quicker the stress will be increased in a film the smaller the critical hydrogen concentrations for buckling. Also a larger hydrogen loss can occur during stepwise loading. The larger hydrogen concentration in the $\mathrm{Nb}$ upper layer and the hydride formation "from top to bottom" of the $\mathrm{Nb}$ film characterises the continuous loading. These differences in hydrogen distribution in metal films by applying of continuous and stepwise hydrogen loadings are more significant for the thicker films and higher loading currents.

The density of the loading current has to be chosen individually for each substrate-film bonding type. Laudahn [Lau98] reported a current density of $j=0.35 \mathrm{~mA} / \mathrm{cm}^{2}$ as optimal. However, this current density is too large for film-substrate systems with small adhesion energy, such as metal films on polymers. It leads to quick stress increase and buckling process on the large area of the film. In this case it is difficult to determine the critical values for buckling with a high accuracy. Therefore, a smaller current density is needed for the weakly adhered systems. In this work $\mathrm{j}=0.15 \mathrm{~mA} / \mathrm{cm}^{2}$ was used for continuous loading. It has shown an optimized buckling-loading relationship for metal films on PC.

\subsection{Polymer substrates: sorption and relaxation}

Secondary effects of hydrogen loading of metal films on polymer substrates with respect to buckling and adhesion energy evaluation are discussed. These effects are the sorption of water from the electrolyte into the polymer and the polymer relaxation during the buckling process.

Since the electrochemical loading was the main hydrogen loading method in this work, the influence of the electrolyte on the measurements was investigated. It was found in Chap. 3.2.1 that water absorption in the polymer substrate is a long-time effect in comparison to the hydrogen loading. Further, the change of stress in the metal-film system is small compared to stress caused by hydrogen loading. The change of stress caused by water absorption is only about $0.7 \%$ of the stress caused by hydrogen loading. But, absorption of water at the interface of the metal and the polymer might weaken the adhesion.

The relaxation of the polymer is related to the delamination of a metal film and can be attributed to the relaxation processes in the polymer below the buckle. This process is clearly visible in the change of stress in the metal-polymer system. However, the physical and chemical details of this process are unknown and should be investigated in future work. 


\subsection{Adhesion energy calculations of different interface layers using the controlled buckling method and buckle geometry}

The adhesion energy evaluation of different metals on PC using the controlled buckling technique and Gille and Rau theory is discussed. The adhesion energy values are compared to literature values.

Surface energies of polymers are small and lay in the range of 10 to $50 \mathrm{~mJ} / \mathrm{m}^{2}$ [L91]. Polycarbonate belongs to the group of polymers with a relatively high surface energy which has been determined by Lee [L68] as $\gamma=42 \mathrm{~mJ} / \mathrm{m}^{2}$. Surface energies of metals are well documented and generally vary between 1 and $5 \mathrm{~J} / \mathrm{m}^{2}$. The surface free energy of $P d$ is $1.90 \mathrm{~J} / \mathrm{m}^{2}$ and 2.05 $\mathrm{J} / \mathrm{m}^{2}$ for $\mathrm{Pd}(001)$ and $\mathrm{Pd}(111)$ respectively [Kim06], [Sir05]. The adhesion energy of $\mathrm{Pd} / \mathrm{PC}$ interface of $\gamma_{\mathrm{Pd} / / \mathrm{PC}}=0.4( \pm 0.1) \mathrm{J} / \mathrm{m}^{2}$ as determined in this work is in-between the surface energy values of $\mathrm{Pd}$ and $\mathrm{PC}$ and therefore in good agreement with the Young-Dupre equation (1.3). For the $\mathrm{Pd} / / \mathrm{Al}_{2} \mathrm{O}_{3}$ interface Hansen [H99] has obtained a value of $\gamma=2.8 \pm 0.2 \mathrm{~J} / \mathrm{m}^{2}$, using a scanning tunnelling microscopy (STM) study of Pd nanoclusters deposited on $\mathrm{Al}_{2} \mathrm{O}_{3}$. Graoui et al. have found an adhesion energy of $\gamma=0.91 \mathrm{~J} / \mathrm{m}^{2}$ for Pd clusters on $\mathrm{MgO}(110)$ [GGH01]. Thus, the adhesion energy of $\mathrm{Pd} / / \mathrm{PC}$ interface obtained in this work using the controlled buckling technique has similar values as the Pd/oxide interface.

The investigation of $\mathrm{Fe}, \mathrm{Nb}, \mathrm{Cr}$ and Mo metal interfaces with $\mathrm{PC}$ show lower adhesion energies than the $\mathrm{Pd} / / \mathrm{PC}$ interface. The lower adhesion can be explained by oxidation of the metal film at its boundary with PC causing the chemical bonds to be broken at this interface. Tan et al. have reported about stress corrosion cracking observed at ACF/metal interface [TCC03]. ACF is a composite material, which consists of an adhesive matrix and conductive particles, which are made of solid metal or metal-coated polymers. PC substrates used in this work need some hours to be pumped down in the pre-chamber before sputtering to achieve a good pressure. That demonstrates the presence of absorbed gases in the polymer. Namely oxygen can react with a metal at the metal-polymer interface.

Abdallah et al. [A06] have found that oxygen plasma treatment of polymer substrates (Arylite ${ }^{\mathrm{TM}}$ ) prior to layer deposition enhances buckling of the $\mathrm{Si}_{3} \mathrm{~N}_{4}$ layer. It can indicate the oxidation of the ceramic layer at the interface and adhesion weakening.

The investigations of a metal surface using white light interferometry in Chap. 3.4.4 have shown the power of this method to obtain the buckle geometry. The resolution was not optimized in these measurements. The use of the ocular with a larger magnification should enhance the 
lateral resolution, which was, in our case, only $1.5 \mu \mathrm{m}$. The vertical resolution of $10 \mathrm{~nm}$ was more than sufficient for these measurements.

Large height variations of about $1 \mu \mathrm{m}$ were detected in the $200 \mathrm{~nm} \mathrm{Nb}$ film at $7 \% \mathrm{H} / \mathrm{Nb}$. Such height variations are typical for the varicose mode of the buckle shape (see Fig. 1.7) with an elastically deformed film in the buckle region. The transition to the telephone cord mode was not observed. Due to the large buckle height variations it is assumed these height variations originate from plastic deformations which occur at strongly bent regions of the buckles such as the buckle rim and the buckle onsets. Also if the hydrogen loading occurs very fast, large plastic deformations are the result of stress release in a metal film. The $50 \mathrm{~nm} \mathrm{Nb}$ film also shows large height variations between $0.4 \mu \mathrm{m}$ and $0.7 \mu \mathrm{m}$ resulting also from the plastic deformations and the increased influence of the $20 \mathrm{~nm} \mathrm{Pd}$ coping layer on the $50 \mathrm{~nm} \mathrm{Nb}$ film in comparison to the films with larger thickness.

The adhesion energy evaluation using white light interferometry data and the theory of Gille and Rau is a very promising method. However, appropriate results can only be achieved analysing buckles of regular shape (e.g. with a sinusoidal profile and constant height along a buckle column). Such buckles were seen for Pd $(10 \mathrm{~nm}) / \mathrm{Nb}(100 \mathrm{~nm}) / \mathrm{Pd}(10 \mathrm{~nm})$ on PC (1mm) at the critical hydrogen concentration of $10 \% \mathrm{H} / \mathrm{Nb}$ as it is shown in Fig. 3.57. The calculated adhesion energy $\gamma=1.05( \pm 0.45) \mathrm{J} / \mathrm{m}^{2}$ is in a good agreement with the adhesion energy value $\gamma_{\mathrm{Pd} / \mathrm{PC}}=0.4( \pm 0.1) \mathrm{J} / \mathrm{m}^{2}$ obtained using the controlled buckling technique.

The elastic energy per area stored in the unbuckled film which was used in the mathematical model of the controlled-buckling technique for the condition of the energetic possibility of buckling can be calculated in two ways: 1. the elastic energy of a film under equi-biaxial compression (Eq. 1.27) with equi-biaxial stress release during buckle formation, 2. The elastic energy per area in the unbuckled film to release under plane strain conditions (Eq. 1.30) used for the Euler mode buckling (uniaxial stress release during buckle formation). In this work straight sided buckles were mostly observed. Therefore, it was favoured the description with uniaxial stress release in the data discussions and Eqs. (1.33) and (1.34) were applied for adhesion energy calculation using the controlled buckling method. However, the adhesion energy calculations applying the elastic energy formulation according to Eq. 1.27 give results which are larger and in better agreement with adhesion energy calculations from the buckle geometry. It is reasonable to assume that every buckle contains plastically deformed regions. Thus, the height-to-width ratio of every real buckle deviates from the theoretical value. It was shown that plastic deformations lead to increase of the value. Thus, applying the formula of Gille and Rau to real buckles always results in adhesion energies which are larger than the real value. 
The adhesion energies calculated assuming a buckle formation according to the Euler mode are about $30 \%$ smaller than the energies calculated using the elastic energy formulation in the unbuckled film in Eq. (1.27). The adhesion energies calculated by using of $\mathrm{c}_{\text {crit }}$ and $\sigma_{\text {crit }}$ as a critical parameter for buckling is similar (see Tab. 3.7).

A bonding weakening effect of about $33 \%$ for $\mathrm{Cu} / / \mathrm{Al}_{2} \mathrm{O}_{3}$ with $1 / 3 \mathrm{ML}$ of interfacial $\mathrm{H}$ is reported [WSS02]. The influence of $\mathrm{H}$ at the interface can be excluded when a non-permeable layer for hydrogen is added to the film package between the $\mathrm{Nb}$ layer and the metal layer, which bonding to the substrate should be measured.

\subsection{Investigation of $\mathrm{Nb} /$ sapphire bonding by using of controlled buckling technique.}

For the investigation of the metal/ceramic adhesion the system $\mathrm{Nb} / \mathrm{Al}_{3} \mathrm{O}_{3}$ was examined. Extraordinary strong adhesion of niobium to sapphire was reported [EKR94], [S97]. The reason for the strong adhesion was explained by the atomic structure of the interface. If the $\mathrm{Al}_{2} \mathrm{O}_{3}$ surface is oxygen terminated, $\mathrm{Nb}$ atoms fill the positions which otherwise would be taken by $\mathrm{Al}$ atoms with formation of a strong ionic bond and large strain amplitudes as it was observed by Song et al. [S97]. Elastic energy can be stored during thin films growth, linearly increasing with increasing film thickness, as it was included in Eq. (1.31). Therefore, $\mathrm{Nb}$ films with large thickness were needed to be able to store enough $\mathrm{H}$-induced elastic for film delamination. No delamination was observed by Laudahn [Lau98] during hydrogen loading of $200 \mathrm{~nm} \mathrm{Nb}$ films on sapphire. In the present work it was possible to detach $\mathrm{Nb}$ films with thickness larger than $4 \mu \mathrm{m}$ from sapphire substrate.

The work of adhesion of the $\mathrm{Nb} /$ sapphire interface of $450 \mathrm{~J} / \mathrm{m}^{2}$ for the $\mathrm{Nb}(110)$ [001]//Sapphire (0001) [1-100] orientation obtained by using the controlled buckling technique in Chap. 3.5.4 is much larger in comparison to the work of separation values of 2.7 to $12.7 \mathrm{~J} / \mathrm{m}^{2}$ which were calculated by Batyrev et al. [B00] using first-principles calculations for nine different combinations of the cleavage planes at the $\mathrm{Nb} /$ sapphire interfaces. But the value of $450 \mathrm{~J} / \mathrm{m}^{2}$ is smaller than the fracture energy $2107 \mathrm{~J} / \mathrm{m}^{2}$ obtained by Elssner [EKR94] for the similar interface orientation of $\mathrm{Nb} /$ sapphire used in this work. The samples in the work of Elssner were prepared by diffusion bonding carried out at $1400^{\circ} \mathrm{C}$ for 3 hours under a compressive stress of $10 \mathrm{MPa}$. The difference in the sample preparation influences the bonding energy of $\mathrm{Nb} /$ sapphire system.

For future investigations of the $\mathrm{Nb}$ /sapphire interface $\mathrm{Nb}$ films with larger thickness could be sputtered. It would be ideal if the film buckling occurs at hydrogen concentrations in the $\alpha$-phase 
range of $\mathrm{H}-\mathrm{Nb}$ system excluding an influence of hydride formation. Taking into account the critical concentrations obtained in this work and the decrease of the $c_{H, \text { crit }}$ by increase of film thickness, the $\mathrm{Nb}$ film thickness for critical hydrogen concentration for buckling $c_{H, c r i t}=6 \% \mathrm{H} / \mathrm{Nb}$ can be calculated. The $\mathrm{Nb}$ film thickness should be about $50 \mu \mathrm{m}$.

\subsection{Single buckling events during hydrogen loading}

The process of single buckle formation can be visualized, as it was shown in Fig. 3.39. Single leaps in the measured mechanical stress, which correlate with buckle formation, were observed during electrochemical hydrogen loading and from the gas phase. The visualisation of single buckle formation event can be compared with single Barkhausen steps [B71] in magnetism.

Many processes take place during formation of buckles which all sum up in the resulting form of the stress curves. Firstly, the formation of buckles results in stress release, which can be observed by the stress reduction in stress curve. Secondly, the buckle formation and stress decrease in the buckle region generate further stress reduction by the Gorsky effect. The Gorsky effect is an inelastic relaxation phenomenon which occurs in metals containing mobile dilatation defects such as hydrogen. If the dilatation gradient occurs in the metal film by buckle formation, the spatial gradient in the chemical potential of the defects will be induced, too. Relaxation will then occur by long-range diffusion of the defects (hydrogen atoms) along this gradient. The diffusion time of hydrogen for reaching a balance of hydrogen concentration between the buckle region and bonded film after buckle formation is theoretical much smaller than $1 \mathrm{~s}$. Gorsky effect leads to stress increase in the buckle region after the buckle formation and further buckle growth. So, the formation of buckles is accompanied by many processes such as dislocations formation and movement during film buckling, hydrogen diffusion into the stress relaxed buckle region and related further extension of the buckle region.

The general development of stress shown in Fig. 3.35 is also affected by many processes such as the polymer relaxation process, formation of new buckles and growth of the already existing buckles. The general stress progression after the onset of buckle formation decreases in the direction of compressive stress. The condition for that is the predominance of the relaxations processes over the compressive stress increase by hydrogen loading. In Fig. 4.1 a sketch of the polymer relaxation process is shown. The polymer swells below the buckled film due to the adhesion forces between the polymer and metal film and buckle formation. The swelling leads to bending of the sample in the direction corresponding to the stress relaxation in the metal film. 


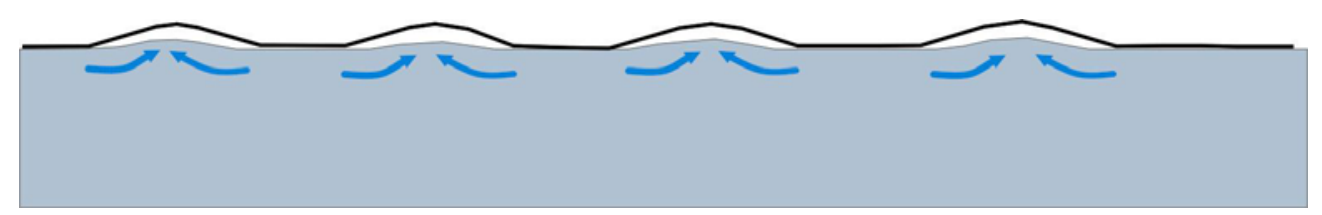

Figure 4.1: Swelling of polymer below a formed buckle. This process can as well be responsible for the bending of a sample in a direction which corresponds to a decrease of the compressive stress in the metal film during hydrogen loading. The change in the polymer morphology was observed in the region below the buckles (see Chap. 3.2.2)

It can also be pointed out that during the buckling processes the saturation limit of hydrogen in the sample increases successive by reducing the mechanical stress in the system and by hydrogen absorption in the regions of a lower stressed film of the buckled region.

\subsection{Hydrogen loading of niobium films on sapphire}

Stress measurement and optical observation of the surface at the same time during hydrogen loading of $\mathrm{Nb}$ films with large thickness have shown large stress relaxation during hydride formation and optically visible hydride formation.

Reasons for the stress relaxation observed during the hydrogen loading were first proposed by Laudahn. Firstly, these are generation and movement of misfit dislocations. Secondly, this is stress induced diffusion of $\mathrm{Nb}$ atoms in grain boundaries to the surface and segregation at the grain boundaries. It can also be assumed that the relaxation process is due to surface hydride formation. The mechanism of such type of relaxation is shown in Fig. 4.2. The volumes of the surface hydrides are pressed out due to the high local compressive stress. According to K. Nörthemann the height change at the surface has a largest value if the hydride is positioned directly under the surface and has a cylindrical form (Fig. 3.20 in [N06]).

a)

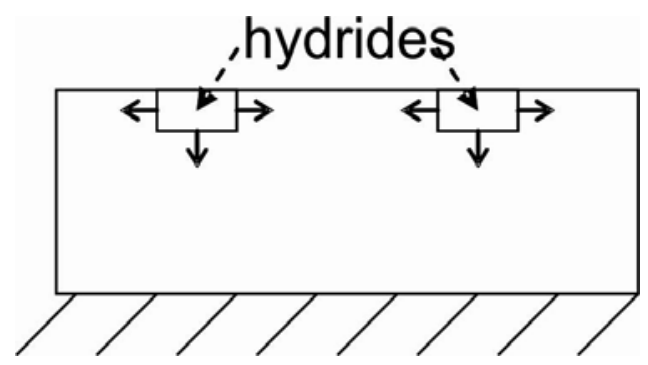

b)

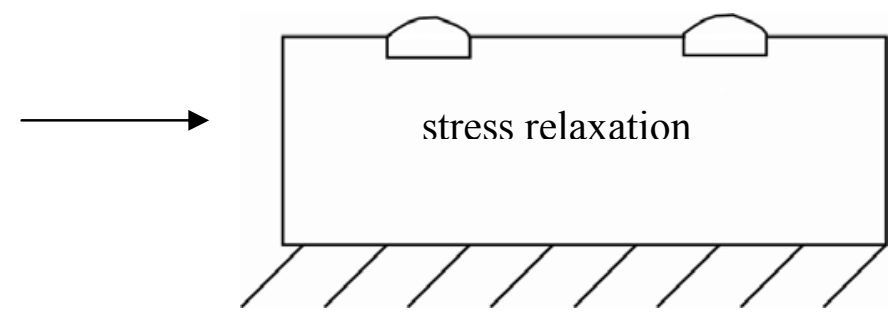

Figure 4.2: Stress relaxation due to surface hydride formation. a) Hydride formation with stress appearance in the vicinity of the hydrides. b) The hydride precipitates are pressed out resulting in stress relaxation.

Morphological changes of the surface were observed by light microscopy for samples with $\mathrm{Nb}$ layer thickness larger than $2 \mu \mathrm{m}$. The appearance of these surface patterns is in correlation with 
inflection points in the stress curves which are correlated to phase transformations. This can be clearly seen in Fig. 3.79 for the $4 \mu \mathrm{m}$ sample during stepwise and continuous hydrogen loading. The second inflection point in each stress curve is shifted to about $6 \% \mathrm{H} / \mathrm{Nb}$ and the formation of new surface patterns was observed with the same concentration difference, what can be seen in Fig. 3.80. This correlation suggests that these surface morphologies are hydride precipitates in the $\mathrm{Nb}$ films. The surface fraction covered with hydride of a $4.75 \mu \mathrm{m} \mathrm{Nb}$ film at $\mathrm{c}_{\mathrm{H}}=12 \% \mathrm{H} / \mathrm{Nb}$ is about $40 \%$ (see Fig. A.4, Appendix A). The $(\alpha, \beta)-\beta$ phase boundary was determined for this film measuring the EMF; it is $43 \% \mathrm{H} / \mathrm{Nb}$. With a simple calculation it can be shown that the hydrides can not be columns through the whole niobium film as it was observed for thinner films [N06]. If the hydride would form in columns ranging through the complete film, the surface coverage of $40 \%$ would result in a mean $\mathrm{H}$-concentration of $23 \% \mathrm{H} / \mathrm{Nb}$. This is well above the measured concentration of $12 \% \mathrm{H} / \mathrm{Nb}$. Therefore it results that the hydride is only partially formed in the film. Most probably it is located at the surface, as shown in Fig. 4.2b. The hydride layer covering the whole sample surface was observed at $18 \% \mathrm{H} / \mathrm{Nb}$ and is schematically shown in Fig. 4.3. From the relationship of hydrogen concentrations in the hydride and $\alpha$-phases of H$\mathrm{Nb}$ system the thickness of the surface hydride layer at $18 \% \mathrm{H} / \mathrm{Nb}$ can be estimated. It is about $1.15 \mu \mathrm{m}$.

a)

b)

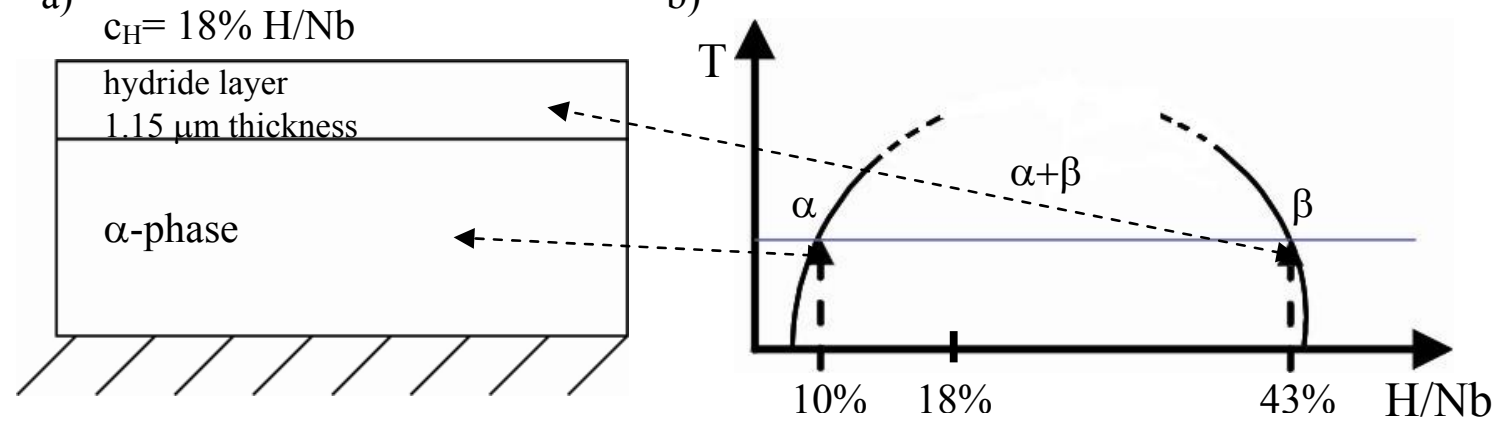

Figure 4.3: Estimation of the thickness of the hydride layer in $4.75 \mu \mathrm{m} \mathrm{Nb}$ film on sapphire substrate at $18 \% \mathrm{H} / \mathrm{Nb}$ from the phase boundaries of the thin film system and the observation of surface covering with the hydride. a) Sketch of the $\alpha$-phase and hydride distribution in the thick $N b$-film. b) Schematical $N b$-H phase diagram with measured phase boundaries.

The fine structures observed on the $\mathrm{Nb}$ surface at $\mathrm{c}_{\mathrm{H}}=32-42 \%$ are linked with the hydridisation of the complete film, transition of cubic structure of $\mathrm{Nb}$ lattice in the $\alpha$-phase to orthorhombic structure in the $\beta$-phase. The resolution of light microscope was about $0.25 \mu \mathrm{m}$. The theoretical total volume increase in the vertical direction which can be achieved in $1.15 \mu \mathrm{m} \mathrm{Nb}$ layer by increase of hydrogen concentration from 0 to $43 \% \mathrm{H} / \mathrm{Nb}$ is $0.065 \mu \mathrm{m}$ (from [Lau98], Eq. 3.14). 
This value is smaller than the resolution of light microscope. Therefore, most probably, the visibility of hydrides is based on the change of optical reflection properties of hydrides.

The upper limit of the hydrogen solubility in niobium was about $80 \% \mathrm{H} / \mathrm{Nb}$. This is smaller than the one obtained by Dornheim [Dor02]. A larger hydrogen loss during the stepwise loading than during continuous loading can be assumed. Continuous electrochemical hydrogen loading using very low current densities should be used in future investigations to achieve larger hydrogen concentrations in $\mathrm{Nb}$ films.

Laudahn [Lau98] has measured an increase of mechanical stress between $-5.7 \mathrm{GPa} / \mathrm{c}_{\mathrm{H}}$ and -9.7 $\mathrm{GPa} / \mathrm{c}_{\mathrm{H}}$ in nanocrystalline and epitaxial Nb-layers on sapphire substrates. The linear elastic model predicts for perfectly adhered $\mathrm{Nb}$ films on substrates an isotropical stress with the slope of $-9.7 \mathrm{GPa} / \mathrm{c}_{\mathrm{H}}$. The slope values measured in this work for $\mathrm{Nb}$ films on sapphire substrates are smaller. They range from $-5 \mathrm{GPa} / \mathrm{c}_{\mathrm{H}}$ to $-6.5 \mathrm{GPa} / \mathrm{c}_{\mathrm{H}}$. The films investigated in this work show (110)-texture, as it is shown in Fig. 3.87. The decrease of hardness leads to a decrease of stress during hydrogen loading. The reasons for anomaly small stress slopes of about $-1 \mathrm{GPa} / \mathrm{c}_{\mathrm{H}}$ by hydrogen loading of $4 \mu \mathrm{m} \mathrm{Nb}$ film could not be clearified. Presumably, the stress measurement for this sample was incorrect. A possible reason for hydrogen losses could be an electrical contact to the metal plate, which was used to enhance the sensitivity of the inductive sensor during the bending measurements. In some samples, the measured resistance between the metal film and the metal plate was about $2.5 \cdot 10^{6} \Omega$. This is quite high resistance. And the measured phase boundaries do not show large hydrogen loss. Nevertheless, one has to be sure in the future that this effect is avoided.

The $\alpha-(\alpha, \beta)$ phase boundary was found at $6.5-11.5 \% \mathrm{H} / \mathrm{Nb}$, which is in good accordance with $6 \% \mathrm{H} / \mathrm{Nb}$ for bulk $\mathrm{Nb}$ and shifting of the phase boundary to higher hydrogen concentrations for thinner films. The $(\alpha, \beta)-\beta$ phase boundary was at $30-43 \% \mathrm{H} / \mathrm{Nb}$. This is much smaller than $72 \%$ for bulk $\mathrm{Nb}$. The reason for that is the high compressive stress in the films, which affect the phase boundaries [Dor02] and leads to narrowing of the two-phase-region. This was also verified in Chap. 3.3, where the influence of stress on the chemical potential was determined. A decrease of the chemical potential in $\mathrm{Nb}$ linked to the onset of buckling and stress relaxation was determined. 


\section{Summary}

In the present work it was shown that buckling of metal films on different substrates can be generated by hydrogen loading of the metal films and, thereby, increasing compressive in-plane stresses. This concept can be applied to all solutes expanding a material which is deposited as a thin film on any other material. However, if the solute induced strain relaxes before the critical stress of detachment is reached or the solubility of the solute in the metal film is low to reach a critical stress, no buckling will be observed. In order to detach a film from its substrate, the critical stress for Euler's instability has to be exceeded. The stress for Euler's instability should be smaller than the critical stress for buckling for adhesion energy measurement. This condition can be easily fulfilled by reducing of film thickness.

Adhesion measurements of different polymer/metal combinations can be performed, because the metal investigated towards adhesion properties can always be deposited as a thin film between polymer and hydrogen absorbing metal film. The investigated metal film should be closed. The bonding of the investigated metal to the hydrogen absorbing metal film should be stronger than to the polymer. For the combination of $\mathrm{Pd} / \mathrm{Nb} / \mathrm{Pd} / \mathrm{PC}$, studied in this work, the $\mathrm{Pd} / / \mathrm{PC}$ interface is the weakest one and hydrogen is absorbed predominantly in niobium.

High compressive stresses by absorption of hydrogen in $\mathrm{Nb}$ films were produced and their relaxation by partial delamination of the film from the substrate with formation and propagation of buckles was investigated. The compressive stress in thin metallic films on polymer substrates was increased stepwise and continuously by electrochemical hydrogen loading and was determined by the substrate curvature experimentally. The critical stress and hydrogen concentration for buckling is larger for thinner films. The adhesion energy was determined by using a critical H-concentration and/or a mechanical stress for buckle formation. Films on rigid substrates should be used for adhesion energy evaluation to avoid elastic energy losses by substrate stretching which lead to wrong critical buckling parameters. The results at different metal/polymer layer systems are presented. For Fe, Nb, Mo, Cr and Pd on PC adhesion energies were determined using the controlled buckling technique. For $\mathrm{Pd}$ on $\mathrm{PC}$ it was $\gamma_{\mathrm{Pd} / \mathrm{PC}}=0.4( \pm 0.1)$ $\mathrm{J} / \mathrm{m}^{2}$. Oxygen from the air and remaining oxygen in the PC react with the metal at the polymer interface leading to much lower adhesion energy values compared to $\mathrm{Pd}$, which is resistant to oxidation. The adhesion energy $\gamma=1.05( \pm 0.45) \mathrm{J} / \mathrm{m}^{2}$ of the $\mathrm{Pd} / / \mathrm{PC}$ interface was also determined according to the theory of Gille and Rau using the geometry of buckles, which was measured applying white-light-interference microscopy. The results of both methods are in good agreement. However, the theory of Gille and Rau is very sensitive to morphological variations of the buckles. 
Large local plastic deformation of films in the buckle regions were observed by exceeding the critical hydrogen concentration for buckling. Thus, the buckle geometry for the adhesion energy calculation has to be measured right at the critical hydrogen concentration at the onset of buckling to minimize plastic deformations content. It is suggested that every real buckle contains plastically deformed regions and, therefore, the adhesion energy values are slightly too large.

It was shown that the critical mechanical stress depends on the loading speed. That indicates a time-dependent component, which has its origin in the behaviour of the polymer. This behaviour was examined in detail and its physical origin was clarified. The origin lays in substantial relaxation processes in the polymer, which particularly takes place underneath the delaminated areas. SIMS investigations show a wrinkle pattern at the polymer surface reproducing the wrinkle pattern of the delaminated film.

The decrease of the chemical potential of hydrogen by onset of buckling was observed, confirming experimentally this dependence and making it possible to apply it as a new method for detecting the first buckles, if the surface observation during stress measurements and the stress measurement are not possible.

A huge adhesion energy of $\mathrm{Nb} /$ /sapphire interface was confirmed by controlled hydrogen loading, by which the detachment of the $\mathrm{Nb}$-films was not possible for film thickness under $4 \mu \mathrm{m}$. Fracture energy of $450 \mathrm{~J} / \mathrm{m}^{2}$ was determined for the given $\mathrm{Nb} /$ sapphire interface. It was also observed that the hydride formation occurs predominantly in the regions of lower stress. Electrochemical hydrogen loading of 0.8-4.7 $\mu \mathrm{m}$ (110)-textured $\mathrm{Nb}$ films on (0001) sapphire substrates shows a large narrowing of $(\alpha+\beta)$ miscibility gap in $\mathrm{Nb}-\mathrm{H}$ system. This is evidence for large stresses introduced by hydrogen loading. Hydride formation was observed using light microscopy. The optical observations of hydride formation are in good correlation with the EMF measurements for the phase boundary determination.

Generally, it can be pointed out that hydrogen loading is a powerful tool to study stress and buckle formation in thin metal films on substrates. 


\section{Appendix A}

\section{A.1 Morphological changes of $\mathrm{Nb}$ surface during hydrogen loading}

Surface monitoring of $\mathrm{Nb}$ films with the thickness from $1.95 \mathrm{~mm}$ to $4.75 \mu \mathrm{m}$ during electrochemical hydrogen loading is performed. Morphological changes which are correlated with the phase transformations of $\mathrm{Nb}-\mathrm{H}$ system were observed.

\section{A.1.1 $1.95 \mu \mathrm{m} \mathrm{Nb}$ film on sapphire}

Morphological changes of the sample surface of $1.95 \mu \mathrm{m} \mathrm{Nb}$ film on sapphire were monitored by light microscopy. The relating images of the surface are shown in Fig. A.1.

a) $9 \%$

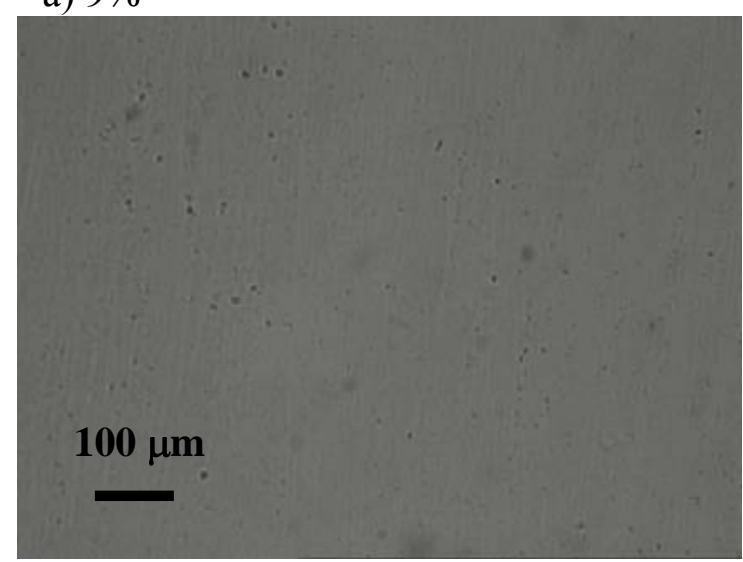

c) $13 \%$

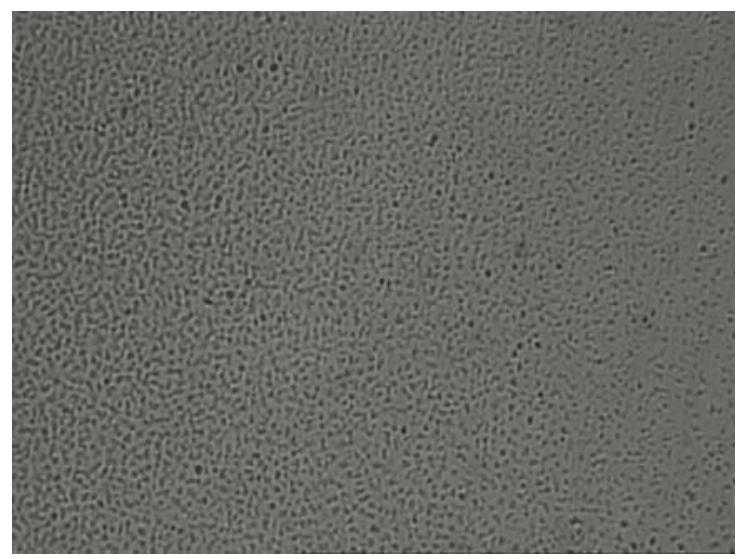

b) $11 \%$

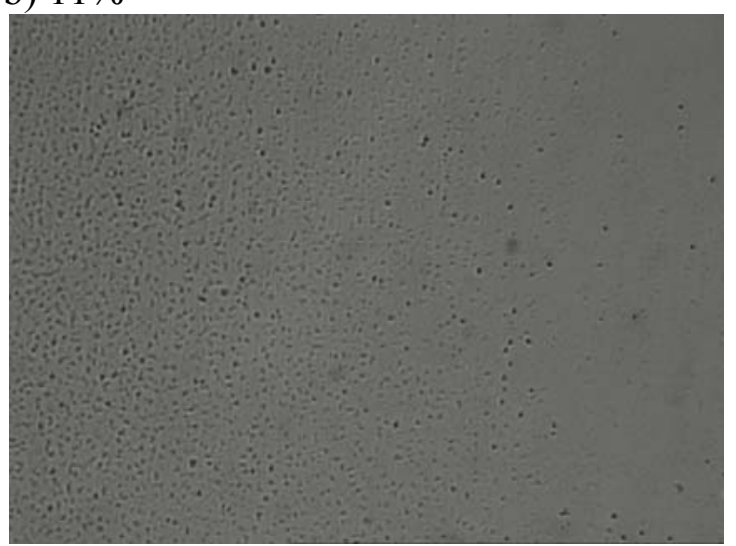

d) $17 \%$

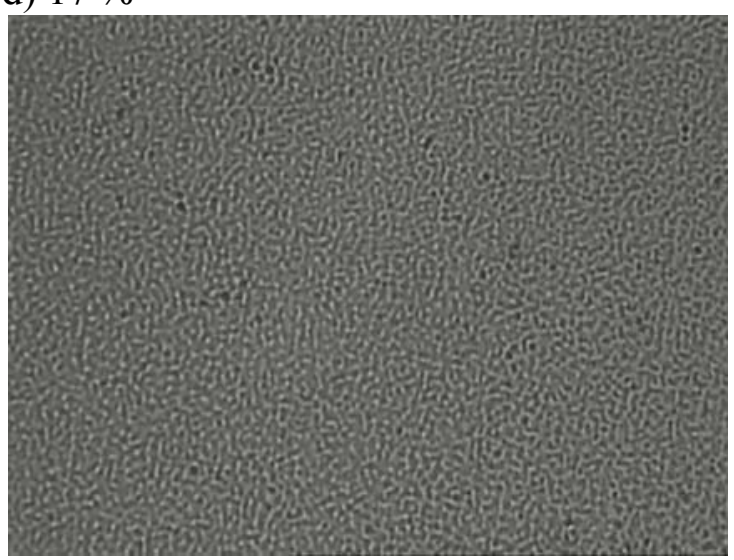


e) $22 \%$

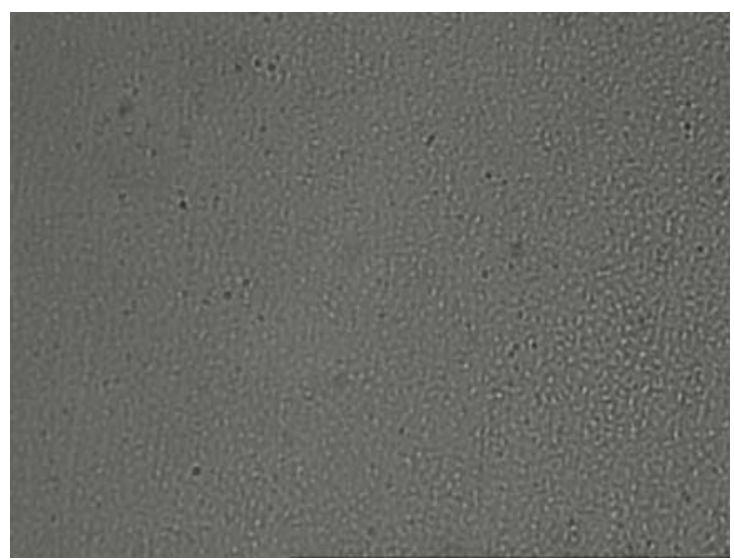

f) $28 \%$

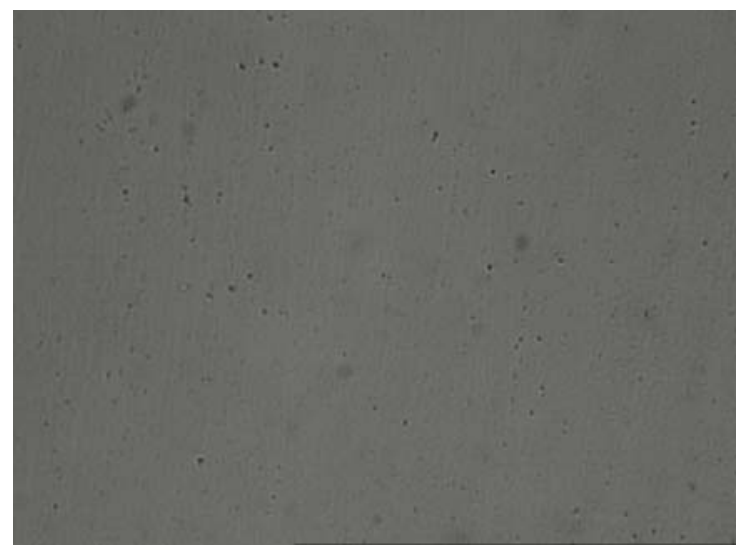

Figure A.1: Light microscopically images of $1.95 \mu \mathrm{m} \mathrm{Nb}$ film on sapphire during the first hydrogen loading sequence. The surface morphology changes observed in the concentration range of 11-23\% $\mathrm{H} / \mathrm{Nb}$ were assumed to be due to formation of niobium hydride.

The changes of the surface morphology at hydrogen concentrations between 10-28 \% $\mathrm{H} / \mathrm{Nb}$ are in good correlation with the existence of the two phase region of $\mathrm{H}$ in $\mathrm{Nb}$. Therefore it can be concluded that the formation of Nb-hydride was observed optically. The height of the $\mathrm{Nb}$ hydrides is expected to be about $45 \mathrm{~nm}$. The lateral sizes of the morphologies, which are visible in Fig. A.1(d), are about 4-5 $\mu \mathrm{m}$. This is much larger than the size of grains, which was calculated applying Scherrers formula to XRD-patterns of the film, which gave about $20 \mathrm{~nm}$.

\section{A.1.2 2.5 $\mu \mathrm{m} \mathrm{Nb}$ film on sapphire}

The light microscopically images of hydrogen loading sequence of the $2.5 \mu \mathrm{m} \mathrm{Nb}$ on sapphire are shown in Fig. A.2.

a) $10 \%$

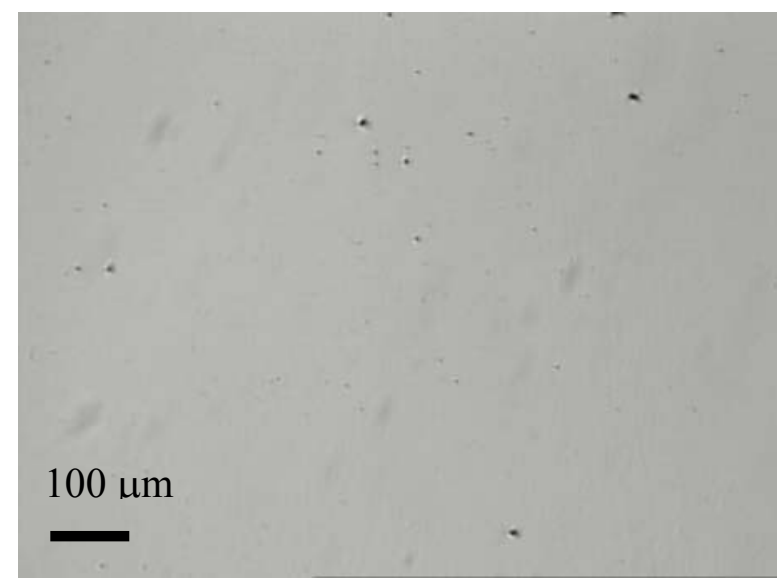

b) $11 \%$

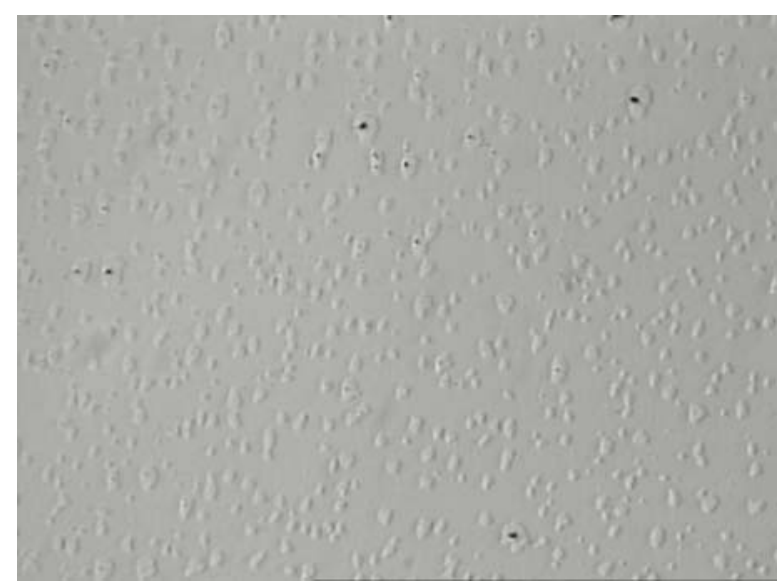



c) $12 \%$

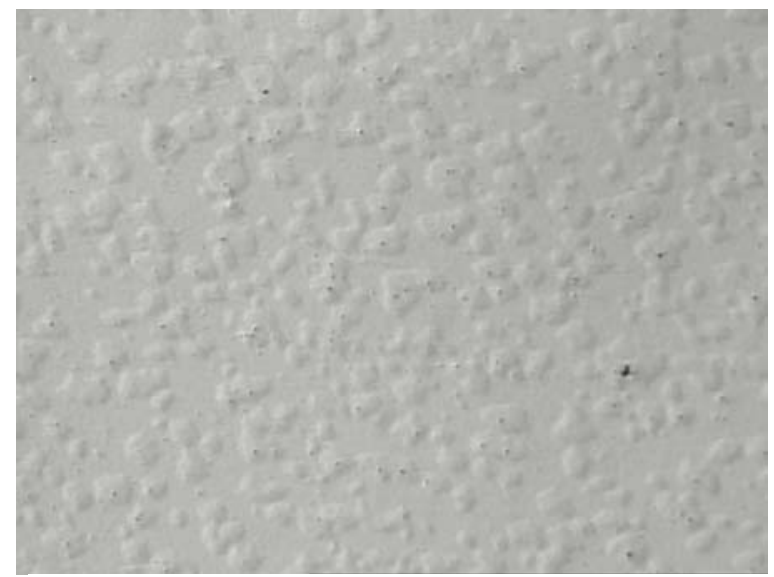

d) $12 \%$ (another place of the sample)

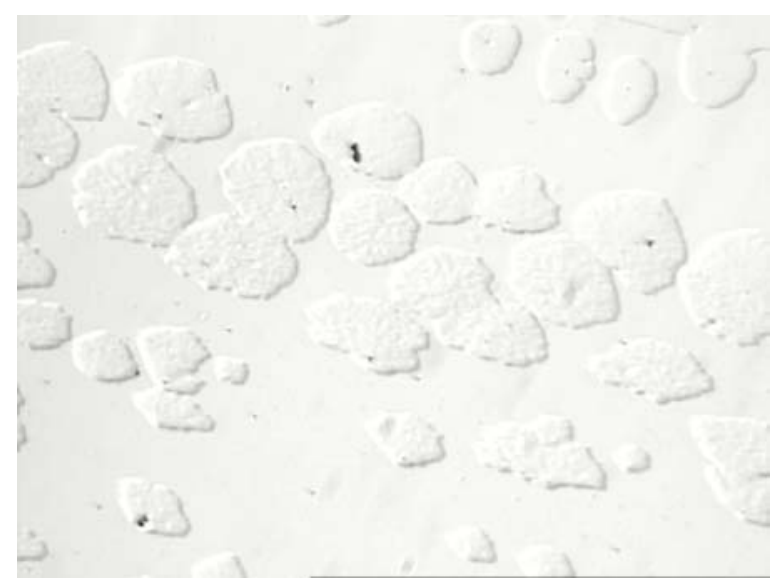

Figure A.2: Light microscopically images of the surface morphology change during hydrogen loading of $2.5 \mu \mathrm{m} \mathrm{Nb}$-layer on $0.2 \mathrm{~mm}$ sapphire substrate.

Again, like for the $1.95 \mu \mathrm{m} \mathrm{Nb}$ sample the surface morphology changes at $11 \% \mathrm{H} / \mathrm{Nb}$. On the other hand, applying SEM it was not possible to see the newly formed regions, probably due to the very smooth slopes between the not-hydrided and the hydrided metal. The further surface morphology changes during hydrogen loading are shown in Fig. A.3.

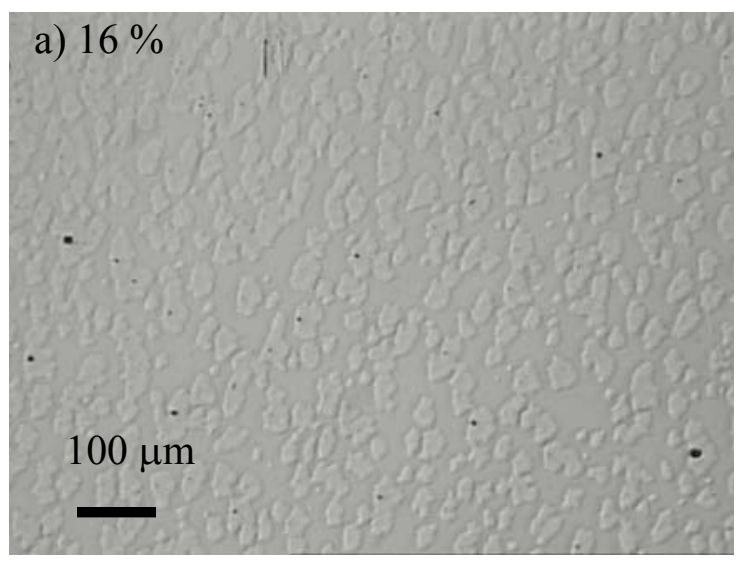

c) $20 \%$

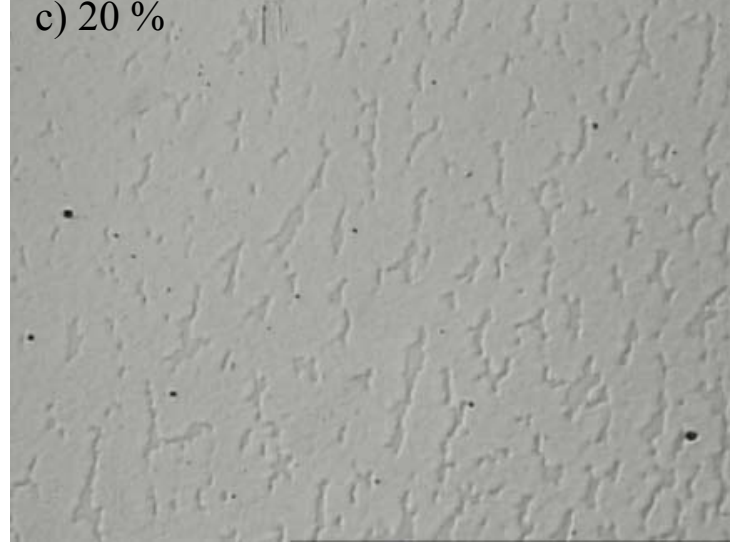

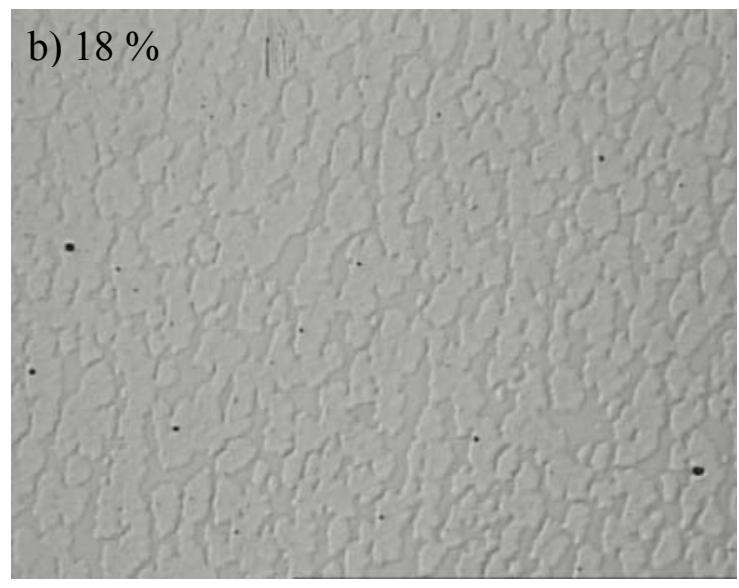

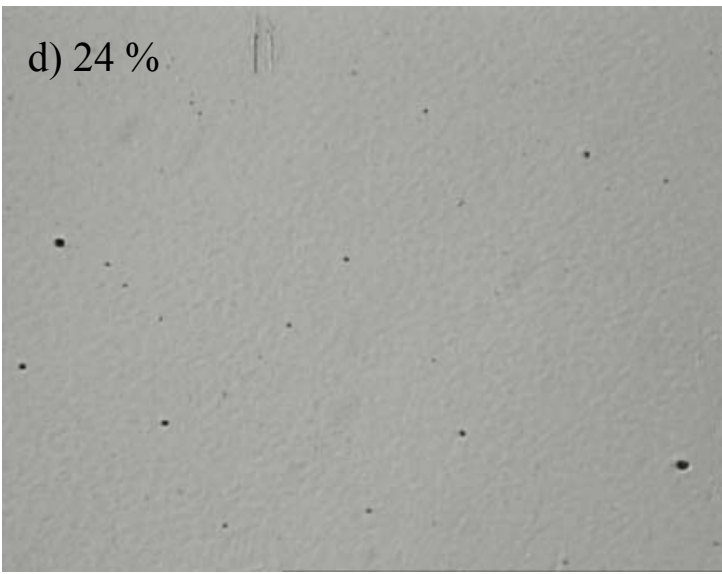

Figure A.3: Light microscopically images of $2.5 \mu \mathrm{m} \mathrm{Nb-layer} \mathrm{on} 0.2 \mathrm{~mm}$ sapphire substrate during hydrogen loading. The hydrogen concentrations are given without taking the small hydrogen loss into account, which is revealed in the emf curve of Fig. 3.77. 
The hydrogen concentrations in Fig. A.3 are given without taking the small hydrogen loss into account, which is present in the emf curve of Fig. 3.77. The newly formed regions, which look light-colored in Fig. A.3(a), grow together and at a nominal concentration of $24 \% \mathrm{H} / \mathrm{Nb}$ the surface looks uniform. It is assumed that at this hydrogen concentration the surface hydride layer is closed. The hydrides do not form throughout the whole niobium film because for this larger H-concentrations are required. It can be only a hydride layer near the surface.

\section{A.1.3 $4.75 \mu \mathrm{m} \mathrm{Nb}$ film on sapphire}

a) $0 \%$

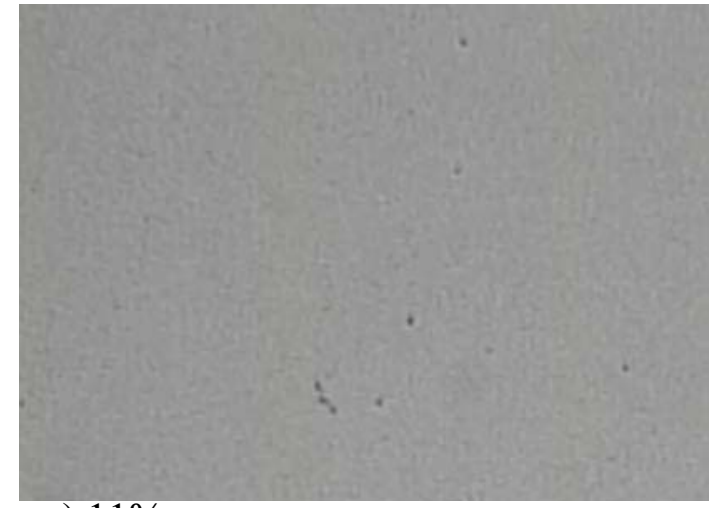

c) $11 \%$

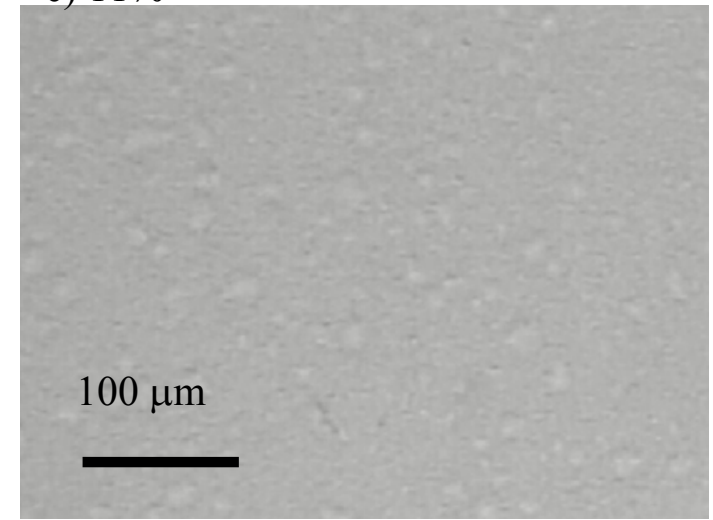

e) $18 \%$ b) $10 \%$

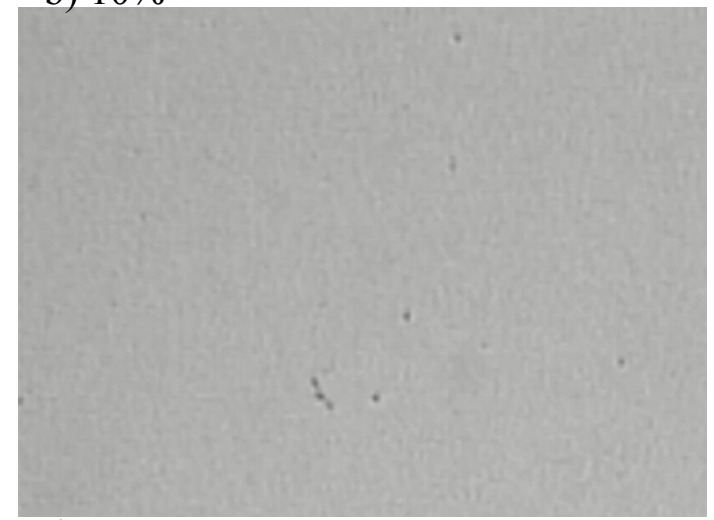

d) $12 \%$

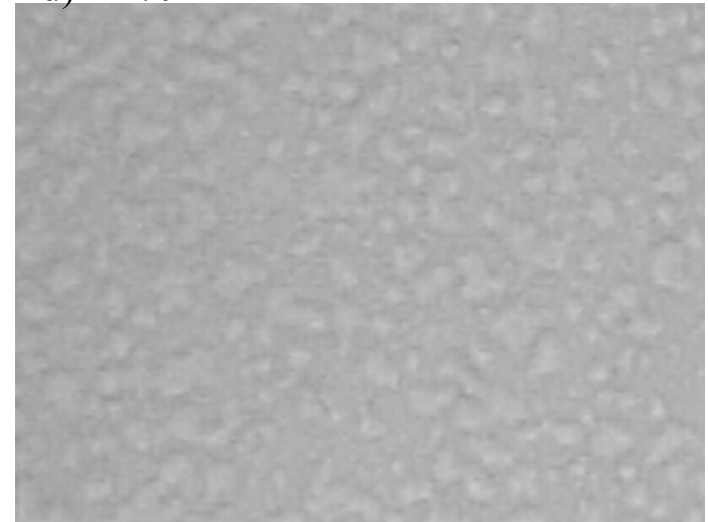

f) $40 \%$

Figure A.4: Light microscopically images of $4.75 \mu \mathrm{m} \mathrm{Nb}$-layer on $0.2 \mathrm{~mm}$ sapphire substrate during stepwise hydrogen loading. a) $0 \% \mathrm{H} / \mathrm{Nb}$, b) $10 \% \mathrm{H} / \mathrm{Nb}$, c) $11 \% \mathrm{H} / \mathrm{Nb}$, d) $12 \% \mathrm{H} / \mathrm{Nb}$, e) $25 \% \mathrm{H} / \mathrm{Nb}$, f) $40 \% \mathrm{H} / \mathrm{Nb}$. 
The first optical changes are visible at $11 \% \mathrm{H} / \mathrm{Nb}$, and at $18 \% \mathrm{H} / \mathrm{Nb}$ the surface is uniform again. Furthermore, other fine structures were observed for $4 \mu \mathrm{m} \mathrm{Nb}$ films at about 36-46\% $\mathrm{H} / \mathrm{Nb}$ and for $4.7 \mu \mathrm{m} \mathrm{Nb}$ films at about 32-42\% $\mathrm{H} / \mathrm{Nb}$. At these hydrogen concentrations a second bending point in the stress curves can be seen (see Fig. 3.79). This region can be correlated to the transition from the $\alpha-\beta$-phase mixture to the $\beta$-phase of Nb-hydride. Two reasons are assumed that could be responsible for the appearance of these structures: a) the surface hydride which covers the total surface of the film at $18 \% \mathrm{H} / \mathrm{Nb}$ dissolves in the lower layers of the film. This process makes again the surface hydride visible at $40 \%$, b) hydridisation of the complete film and transition of cubic structure of $\mathrm{Nb}$ lattice in the $\alpha$-phase to orthorhombic structure in the $\beta$-phase produce stresses in the film and lattice deformations.

The hydrogen concentrations regions, at which visible changes were observed on the surface by light microscopy are given in Tab. A.1.

Table A.1: Hydrogen concentrations at which optical changes (hydride formation) were observed using light microscopy.

\begin{tabular}{|c|c|c|c|c|c|}
\hline $\begin{array}{c}\text { Nb film } \\
\text { thickness, } \\
\mu \mathrm{m}\end{array}$ & 0.8 & 2 & 2.5 & 4 & 4.7 \\
\hline$\Delta \mathrm{c}_{\mathrm{H} / \mathrm{Nb}, \%}$ & - & $11-28$ & $11-23$ & $11-24$ & $11-18$ \\
\hline
\end{tabular}




\section{References}

[ASTM] ASTM D907-70, Definition of Terms Relating to Adhesion, American Society for Testing and Materials.

[A06] Abdallah A. A. et al, Thin Solid Films 503, 167-176, 2006.

[AV78] Alefeld G., Völk1 J., in Hydrogen in Metals I, edited by G. Alefeld and J. Völk1, Springer-Verlag, Berlin, 1978.

[B00] Batyrev I. G., Alavi A. and Finnis M. W., Equilibrium and adhesion of Nb/sapphire: The effect of oxygen partial pressure Phys. Rev. B 62 468-4706, 2000.

[B71] Becker J. J., J. Appl. Phys. 42 1537, 1971.

[B98] Bicker M. et al. Rev Sci Instrum, 69:460, 1998.

[B65] Bockris J. O’M, McBreen J., Nanis L., J. Electrochem. Soc., 112, 1025, 1965.

[BI89] Brandrup J., H.Immergut E., Polymer Handbook, 1989.

[C98] T. Coffey et al. "X-ray radiation damage of polymers in a scanning transmission xray microscope.” In EXPO: Microscopy and Microanalysis, Atlanta, Georgia. Springer-Verlag (Conference Proceedings), 1998.

[CNC99] Coupeau C., Naud J.F., Cleymand F., Goudeau P.and Grilhé J., Thin Solid Films 353, 194, 1999.

[DG94] Deck L., P. de Groot, Appl. Opt., 33:7334-7338, 1994.

[DMT75] Derjaugin B. V., Muller V. M., Toporov Y. P., J. Colloid Interface Sci., 53, $314-$ 326, 1975.

[Dor02] Dornheim Martin, Spannungen, Dehnungen und Lage der Phasengrenzen in dünnen $\mathrm{Nb}$ - und Y-Schichten bei Wasserstoffbe- and - entladung. Dissertation, Institut für Materialphysik, Uni. Göttingen, 2002.

[DKG03] Droste U., Körner K., Goretzky A., Osten W., Lehmann P., Annual Report Inst. Techn. Optik, Universität Stuttgart, 2003/2004.

[EKR94] Elssner G., Korn D. and M. Rühle Scripta Metallurgica et Materialia, Vol. 31, No. 8, pp. 1037-1042, 1994.

[G07] Gemma R., Al-Kassab T., Kirchheim R., Pundt A., Journal of Alloys and Compounds 446-447, p534-538, 2007.

[GR84] Gille G, Rau B. Thin Solid Films, 120,109, 1984.

[GGH01] Graoui H., Giorgio S., Henry C.R., Philos. Mag. B 81, 1649, 2001. 
[GS05] Grundmeier G., Stratmann M.,Adhesion and de-adhesion mechanisms at polymer/metal interfaces, Annu. Rev. Mater. Res. 35, 571-615, 2005.

[H99] Hansen K. H. et al., Phys. Rev Lett. 83, 4120, 1999.

[H96] Huiberts J. N. et al, Nature (London) 380, 231, 1996.

[HS92] Hutchinson J.W., Suo Z., Adv Appl Mech; 29:63, 1992.

[JKR71] Johnson K. L., Kendall K., Roberts A. D., Proc. Roy. Soc. A., 324, 301-313, 1971.

[K71] Kendall K., J. Phys. D: Appl. Phys., Vol. 4, 1971.

[Kim06] Kim S. H. et al. Journal of the Korean Physical Society, 49, 4, October, 1524-1529, 2006.

[KL80] Kirchheim R., McLellan R.B., J. Electrochem. Soc. 27, 11, 2419-2425

[KSSch82] Kirchheim R., Sommer F., Schluckebier G., Acta Metall,30:1059,1982.

[K00] Klein C. A., J. of Appl. Phys., 88, 5487-5489, 2000.

[LL86] Landau L., Lifshitz E., Theory of Elasticity. Oxford: Pergamon Press; 1986.

[Lau98] Laudahn U.: Spannungen und Dehnungen von mit $\mathrm{H}$ beladenen Nb-Einfach und Pd-Nb-Vielfachschichten., Universitaet Göttingen, Dissertation, 1998.

[LPB99] Laudahn U., Pundt A., Bicker M., von Hülsen U., Kirchheim R., et al. J Alloys Comp;293:490-94, 1999.

[LL94] Lee C., Lin K., Japan J Appl Phys 33:2684, 1994.

[L91] Lee Lieng-Huang, Fundamentals of Adhesion, Springer Verlag, ISBN 0306434709, 1991.

[L68] Lee L. H., J. Appl. Polym. Sci. 12, 719, 1968.

[LCE98] Lipkin D.M., Clarke D.R., Evans A.G., Acta Mater, 46:4835, 1998.

[M92] Maugis D.J., J. Colloid. Interface Sci. 150, 243, 1992.

[MBK81] Matuda N., Baba S., Kinbara A., Thin Solid Films 81, 301, 1981.

[M95] Mittal K. L., Adhesion measurement of films and coatings, VSP, Utrecht, p. 5, 1995.

[M02] Moharram M. A et al, J. of Appl. Polymer Science., 85, Iss.8, 1619-1623, 2002

[M04] Moon M-W et al, Acta Mat 52: 3151-3159, 2004. 
[NKP05] Nikitin E., Kirchheim R., Pundt A. Determination of adhesion energies by means of hydrogen loading, Journal of Alloys and Compounds 404-406, p 477-480, 2005.

[Not95] Notten P. H. L.: in Interstitial Intermetallic Alloys, 281, Chap. 7.

[N06] Nörthemann K., Wasserstoffabsorption in epitaktischen Niobschichten: eine STMStudie. Dissertation, Institut für Materialphysik, Uni. Göttingen, 2006.

[O94] van Oss C.J., Interfacial Forces in Aqueous Media, Marcel Dekker, New York, 1994, ISBN 0-8274-9168-1

[P78] Peisl H., Hydrogen in Metals I. Topics in Applied Physics Vol. 28. Herausgegeben von G. Alefeld und J. Voelkl, Springer-Verlag, Berlin, p. 53, 1978.

[PNPK04] Pundt A., Nikitin E., Pekarski P., Kirchheim R., Acta Mat., 52, 1579-1587, 2004.

[P07] Pundt A., Brekerbohm L., Niehues J., Wilbrandt P-J., Nikitin E, Adhesion-energy measurements by means of white-light interferometry and controlled-buckling technique. Scr. Mat. 57, 889-892, 2007.

[PGB00] Pundt A, Getzlaff M, Bode M, Wiesendanger R, Kirchheim R. Phys Rev B, 61:9964, 2000.

[PLG00] Pundt A, Laudahn U, Geyer U, Getzlaff M, Bode M, Wiesendanger R, Kirchheim R. Mater Res Soc Symp Proc, 594:75, 2000.

[RDR90] Reimanis I. et al, Acta Metall, 38:2645, 1990.

[S05] Siau S. et al, Journal of The Electrochemical Society, 152 (6), C442-C455, 2005.

[Sir05] de Siervo A. et al. Surface Science, 575, iss. 1-2, 217-222, 2005.

[San99] Sander D., The correlation between mechanical stress and magnetic anisotropy in ultrathin films. Rep. Prog. Phys., 62:809-858, 1999.

[S05] Siau S. et al, Journal of The Electrochemical Society, 152 (6) 442-455, 2005.

[SchW78] Schober T., Wenzl H. in: Hydrogen in Metals II. Topics in Applied Physics 29, Springer-Verlag, Berlin, p. 12, 1978.

[Sht03] Shtaya M: Size- selective syntesis of nanometer-sized palladium clusters and their hydrogen salvation behaviour, Diss. Univ. Göttingen, 2003.

[S97] Song G., Remhof A., Theis-Bröhl K., Zabel H., Phys. Rev. Let. 79, 25, 5062-5065, 1997.

[SH88] Steinmann P.A., Hintermann H.E. J. Vac. Sci. Technol. A 7(3), 1989

[Sto09] Stoney G.G., Proc. Roy. Soc. London A 82, 172, 1909.

[TCC03] Tan C.W., Chiu Y.W., Chan Y.C., Mat. Sci. and Eng. B98m, 255- 264, 2003. 
[VKG93] Venkataraman S. K., Kohlstedt D. L.,Gerberich W.W., Thin Solid Films, 223: 269$275,1993$.

[VMG02] Volinsky A. A., MoodyN. R., Gerberich W. W., Acta Mat., 50, 441-466, 2002.

[VRS98] Vitos L., Ruban A.V., Skriver H.L., Kollar J., Surface Science 411 186-202, 1998.

[W08] Walther F., Stability of the hydrophilic behaviour... J. Micromech. Microeng. (In press)

[WSS02] Wang X.-G., Smith J. R., Scheffler M., Phys. Rev. B 66, 073411, 2002.

[W82] Wenzl H., Int. Metall. Rev. 27, 140, 1982.

[WR70] Wiswall R. H., Reilly Jr. J. J.: The Higher Hydrides of Vanadium and Niobium. Inorganic Chemistry, 9:1678-1682, 1970.

[Wag05] Wagner S., Untersuchung der Leitungsmechanismen in wachsenden Palladiumschichten und Veränderung durch Wasserstoffbeladung, diploma thesis, Institut für Materialphysik, University Göttingen, 2005.

[WQL06] Wang H., Qian M.L. and Liu W., Ultrasoonics, v. 44 suppl. 1, 1349-1353, 2006.

[Y96] Yang Q. M, Schmitz G., Fa“hler S., Krebs H. U., and R. Kirchheim, Phys. Rev. B., V. 54, 13, 9131-9140, 1996.

[YH98] Yueguang W., Hutchinson J. W., International Journal of Fracture 93, 315-333, 1998.

[YH02] Yu H. H, Hutchinson J.W., Int J Fract, 113, 39-55, 2002. 
Danksagung

Ich möchte mich bei Herrn Prof. Dr. Kirchheim für die Möglichkeit bedanken, meine Doktorarbeit am Institut für Materialphysik durchzuführen. Vielen Dank für sachliche Diskussionen und richtungweisende Ratschläge.

Frau Prof. Dr. Astrid Pundt bin ich sehr dankbar für die Betreuung dieser Arbeit mit vielen anregenden Diskussionen und freundlicher Unterstützung.

Allen Mitgliedern der Wasserstoff-Gruppe (auch Ehemaligen) danke ich für die freundliche Arbeitsatmosphäre, Unterstützung und Hilfe bei den Messreisen. Ein besonderer Dank gilt Stefan Wagner für das Korrekturlesen dieser Arbeit.

Eine große Hilfe bei der Anfertigung der Arbeit war die stetige Bereitschaft des technischen Personals des Instituts zu professioneller Hilfe bei Messungen und beim Gerätebau.

Für die Zusammenarbeit bei der Probenpräparation und bei Messungen danke ich Dr. Jörg Hoffman, der Gruppe von PD Dr.-Ing. Guido Grundmeier (MPI für Eisenforschung, Düsseldorf) und Herrn Prof. Dr. Franz Faupel (Universität Kiel).

Vielen Dank an meiner Familie, die mit Ihrer Geduld und Liebe meine Arbeit unterstützt hat. 


\section{Lebenslauf}

\section{Persönliche Daten:}

Name:

Eugen Nikitin

Geburtsdatum und - ort:

Staatsangehörigkeit:

Familienstand:

08.02.1973, Ust-Kamenogorsk / Kasachstan deutsch

verheiratet, 2 Kinder

Schulbildung:

$1980-1990$

Mittelschule in Ust-Kamenogorsk/Kasachstan

Studium:

1991-1996

Ost-Kasachstanische staatliche Universität in Ust-Kamenogorsk; Abschluss als Physikingenieur

1996-1998

MSc in Physik

$1998-2000$

Doktorand an der Ost-Kasachischen Universität

seit $07 / 2001$

Promotionsstudiengang am Institut für Materialphysik in der Arbeitsgruppe von PD. Dr. A. Pundt

Berufstätigkeit:

$1998-2000$

Physiklehrer in einer Mittelschule and an der Ost- Kasachstanischen staatlichen Universität

seit $07 / 2001$

Wissenschaftlicher Mitarbeiter am Institut für Materialphysik, Goettingen 